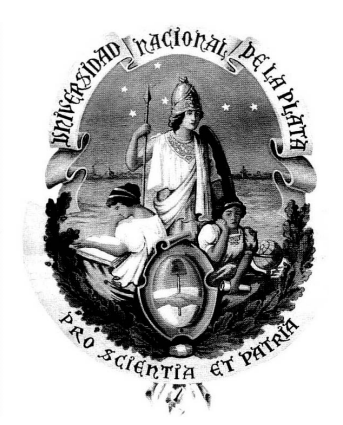

\author{
Universidad Nacional de La Plata \\ Facultad de Ciencias Astronómicas y Geofísicas
}

Tesis para obtener el grado académico de Doctor en Geofísica

\title{
CARACTERIZACIÓN ISOSTÁTICA DEL SECTOR VOLCÁNICO DEL MARGEN CONTINENTAL ARGENTINO
}

Ana Carolina Pedraza De Marchi

Directora: Dra. Marta E. Ghidella

Co-directora: Dra. Claudia N. Tocho 

CARACTERIZACIÓN ISOSTÁTICA DEL SECTOR VOLCÁNICO DEL MARGEN CONTINENTAL ARGENTINO

TESIS PRESENTADA EN CUMPLIMIENTO DE LOS REQUISITOS PARA OBTENER EL GRADO DE

Doctor en GeofísicA

en la

Facultad de Ciencias Astronómicas y Geofísicas

Universidad Nacional de La Plata

Por: Ana Carolina Pedraza De Marchi

Directora: Dra. Marta E. Ghidella

co-Directora: Dra. Claudia N. Tocho

Jurado: Dra. Claudia L. Ravazzoli

Dra. Claudia B. Prezzi

Dr. J. Francisco Ruiz

Facultad de Ciencias Astronómicas y Geofísicas

Universidad Nacional de La Plata

Paseo del Bosque S/N

(1900) La Plata

Argentina

Marzo 2015

(C) Ana Carolina Predraza De Marchi, 2016. 



\section{Prefacio}

Esta Tesis es presentada como parte de los requisitos para obtener el grado académico de Doctor en Geofísica de la Universidad Nacional de La Plata. La misma contiene los resultados de los estudios desarrollados bajo la dirección de la Dra. Marta E. Ghidella del Instituto Antártico Argentino y la co-dirección de Claudia N. Tocho de la Facultad de Ciencias Astronómicas y Geofísicas de la Universidad Nacional de La Plata, entre los años 2010 y 2014.

Ana Carolina Pedraza De Marchi. e-mail: cpedrazadm@fcaglp.unlp.edu.ar La Plata, Marzo de 2015. 



\section{Resumen}

La idea general en la que se basa esta tesis es encontrar una relación entre las anomalías de gravedad y las características volcánicas del sector del margen continental argentino, utilizando datos de anomalías de gravedad derivadas de misiones de altimetría satelital, de anomalías de gravedad marina y datos de espesor sedimentario para estimar la anomalía isostática con diferentes modelos y metodologías que aportan a la caracterización gravimétrica de la región. Inicialmente se emprendió la tarea de organizar los datos de anomalías gravimétricas de aire libre en la región y compilar los datos en una base de datos consistente y documentada. Para ello se compararon grillas de anomalías de gravedad de aire libre derivadas de misiones de altimetría satelital con anomalías de gravedad marina calculadas a partir de datos de cruceros en el margen argentino y zona oceánica adyacente. Se analizaron los datos de barco de distintas campañas realizadas en épocas diferentes y en una primera etapa se organizaron en una base de datos común, a partir de la cual se crearán versiones mejoradas con nuevas correcciones.

Otro objetivo específico consistió en comparar el cálculo de la anomalía de Bouguer con dos métodos. El primer método utiliza la relación entre topografía y gravedad con el desarrollo de Parker en el dominio de Fourier y el segundo método calcula la anomalía de Bouguer completa (Bullard A, B o C) en varios rangos de distancias con diferentes aproximaciones para la atracción de prismas rectangulares y cónicos en las cercanías de la estación. El propósito de este análisis fue validar una metodología de cálculo para la anomalía isostática utilizando transformada de Fourier, en una amplia región del mar argentino con el esquema de Airy.

Una vez validada la metodología de cálculo, se trabajó en otro de los objetivos específicos, que consistió en investigar el mecanismo de compensación de tipo Airy con un esquema de tres interfaces utilizando las metodologías que se describen a continuación: 1) en sentido directo con diferentes aproximaciones del desarrollo de Parker a través del cálculo de tres interfaces con compensación isostática local.

2) invirtiendo la anomalía obtenida en 1), determinando la deflexión del Moho necesaria para compensarla (anularla) utilizando el mismo esquema de tres interfaces y un método iterativo con más términos en la inversión. La interfaz corteza-manto (Moho) así calculada representa una superficie más realista que la calculada con un término en la inversión y que la estimada con datos de topografía y espesor sedimentario, pero no deja de ser un ensayo para probar los métodos numéricos.

Si bien el experimento constituye una suposición esquemática para probar los métodos numéricos, encontramos que en la comparación con el único perfil de refracción digitalizado disponible, la interfaz "Moho" invertida reproduce bastante bien el Moho modelado en dicho perfil, para el caso del método iterativo. 
Luego se ha focalizado en la aplicación de las técnicas backstripping y Modelado de Gravedad Orientado por los Procesos (POGM, Process Oriented Gravity Modelling). El backstripping es una técnica cuantitativa basada en la remoción de la carga desde el basamento, aplicada bajo las hipótesis de isostasia de Airy o flexural, asumiendo conocida la carga sobre la corteza ejercida por el agua y los sedimentos, lo que supone que conocemos el espesor sedimentario. La grilla de espesor sedimentario utilizada ha sido compilada de varias fuentes de datos, resultado de digitalizaciones de mapas publicados y tiempo de ida y vuelta de la señal sísmica, con una densidad constante y no incorpora datos de estratigrafía de pozos.

A partir de la ecuación del backstripping es posible determinar la subsidencia tectónica directamente desde datos estratigráficos. Dicha ecuación consta de tres términos: el primer término (a), tiene en cuenta la profundidad del agua, el segundo (b), considera la carga de los sedimentos y el tercero considera la carga del nivel del mar (c). Aplicamos la técnica backstripping a perfiles y así pudimos estimar la profundidad del basamento en ausencia de sedimentos, es decir la paleobatimetría en el tiempo del rift, que ha sido calculada teniendo en cuenta todo el espesor de sedimentos sin diferenciación de edades. En esta etapa no se han hecho consideraciones acerca de la porosidad de los sedimentos, parámetro que entra en juego en los procesos de compactación y descompactación. Para el caso de backstripping flexural se hacen las mismas suposiciones que para el caso de Airy y se define el parámetro apropiado en el espacio de los números de onda que modifica la respuesta de Airy produciendo la respuesta flexural.

El Modelado de Gravedad Orientado por los Procesos combina la técnica backstripping con técnicas de modelado de gravedad. Utiliza información sísmica del espesor sedimentario y del volcanismo presente (SDRs (por sus siglas en ingés: Seaward Dipping Reflectors) e intraplaca) en la configuración del margen actual, para estimar la anomalía de gravedad que está asociada a los procesos: rifting, sedimentación y magmatismo. El objetivo de POGM es discriminar la contribución que los diferentes procesos geológicos aportan a la anomalía observada y el objetivo del backstripping es determinar la geometría del margen al tiempo del rift. Se considera que los resultados obtenidos en esta Tesis son ampliamente satisfactorios y que el método constituye la base necesaria para una buena interpretación de las anomalías de gravedad en las regiones marinas. Aunque en esta Tesis se han obtenido resultados interesantes para el análisis de la isostasia, no hemos llegado a una caracterización amplia de los márgenes volcánicos a partir de la gravedad. Consideramos que hace falta más trabajo de experimentación y análisis geológico. De todos modos la puesta a punto de los métodos de cálculo, la ejercitación y análisis de los mismos con datos reales constituye un aporte muy valioso para el estudio del margen argentino. 


\section{Abstract}

The general idea on which this thesis is based is to find a relationship between gravity anomalies and the volcanic characteristics of the Argentine continental margin, using gravity anomaly data derived from satellite altimetry missions, of marine gravity anomalies and sedimentary thickness data. The main objective is to estimate the isostatic anomaly with different models and methodologies that contribute to the gravimetric characterization of the region.

Initially, we addressed the task of organizing the free air gravimetric anomaly data in the region and compiling a consistent and documented database. In order to achieve this the free air gravity anomaly grids derived from satellite altimetry missions with the marine gravity anomalies calculated from cruise data in the Argentine margin and the adjacent ocean area were compared. Ship data from different campaigns at different times were analyzed, and in the first stage were organized into a common database, which would be the starting point to create improved versions with potential new corrections.

Another specific objective was to compare the calculation of the Bouguer anomaly with two methods. The first method uses the relationship between topography and gravity with Parker's development in the Fourier domain, and the second one calculates the complete Bouguer anomaly (Bullard A, B or $\mathrm{C}$ ) in various ranges of distances with different approximations to the attraction of rectangular and conical prisms near the station. The purpose of this analysis was to validate a method for isostatic anomaly calculation, by means of the discrete Fourier transform and the Airy scheme, in a wide region of the Argentine sea.

Once the calculation method was validated, we worked on another specific objective, which was to investigate the mechanism of compensation of the Airy type with a scheme of three interfaces by using the methodology described below:

1) In the forward direction by using different approximations and calculating Parker's development through three interfaces with local isostatic compensation.

2) By inverting the anomaly obtained in 1) and determining the deflection of Moho necessary to compensate it (cancel it) using the same scheme of three interfaces.

3) By applying an iterative method with more terms in the inversion. The crust-mantle interface (Moho) thus calculated represents a more realistic surface than that calculated with one term in the inversion and also than that estimated with topography and sedimentary thickness data. Even though the experiment was focused on testing numerical algorithms we found that in comparison with the single seismic refraction digitized profile available the inverted "Moho" interface reproduces quite well the Moho on that profile for the iterative method.

Then the focus was on the application of backstripping techniques and "process ori- 
ented" (POGM, Gravity Oriented Process Modelling) gravity modelling. Backstripping is a quantitative technique based on the removal of loads from the basement, applied on the assumption that there is Airy or flexural isostasy, assuming we know the load on the crust exerted by the water and sediment, which means we know the sedimentary thickness. The grid of sedimentary thickness used has been compiled from various data sources, results from scans of published maps and the two-way time of the seismic signal with a constant density and does not include stratigraphy well data.

From the backstripping equation it is possible to determine the tectonic subsidence directly from stratigraphic data. This equation consists of three terms: the first term (a) takes into account the depth of water, the second (b) considers the sediment loading and the third considers the sea level loading (c). We applied the backstripping technique to profiles and thus we could estimate the depth of the basement in the absence of sediment, i.e. the palaeobathymetry at the time of rifting, which was calculated taking into account the entire thickness of sediments without age differentiation. Considerations about the porosity of the sediments have not been made, a parameter which is part of the compaction and decompression process. In the case of flexural backstripping, the same assumptions as in the Airy case are made, and the appropriate parameter is defined in the wavenumber space that modifies the Airy response producing the flexural response.

The process oriented gravity modelling combines the backstripping technique with techniques of gravity modelling. It uses seismic information of the sedimentary thickness and present volcanism: Seaward Dipping Reflectors (SDRs) and intraplate, in shaping the current margin to estimate the gravity anomaly that is associated with rifting, sedimentation and magmatic processes. The objective of POGM is to discriminate how different geological processes contribute to the observed anomaly and the aim of backstripping is to determine the geometry of the margin at the time of rifting. We considered that the results obtained in this Thesis are highly satisfactory and the method employed allows for a good interpretation of gravity anomalies in marine regions.

Although interesting results for isostasy analysis have been obtained in this Thesis, we have not reached a broad characterization of volcanic margins from gravity. We believe that more work in this direction coupled with geological analysis has still to be done. Nevertheless, the development of methods of calculation and the exercise and analysis using them with real data is a valuable contribution to the study of the Argentine margin. 


\section{Agradecimientos}

A mis padres Antonieta y Miguel por transmitirme la vida.

A mis hermanas Laura y Natalia por estar siempre. A mis sobrinos Lisandro, Agustina, Simón y Tomás.

A mis tíos Jorge y Zulma. A mi tía Coty y primos Estani y Lauti.

A Ceci y Cami.

A los amigos que supieron acompañarme, Paula, Estela, Claudia, Viviana, Lola y Marcos.

A mi directora Marta Ghidella y codirectora Claudia Tocho por su tiempo y dedicación para guiarme en estos 5 años.

A Claudia Ravazolli, Caludia Prezzi y Francisco Ruiz por integrar el jurado y brindarme sus sugerencias, correcciones y observaciones.

A los profesores de la facultad y en especial a Claudio Brunini, Luis Guarracino, Julio Gianibelli, Eduardo Corti y Eduardo Agosta.

A Eduardo Suarez por su ayuda con GMT.

A la facultad de Ciencias Astronómicas y Geofísicas por formarme en el desarrollo académico y cederme un lugar de trabajo.

A CONICET por financiar los estudios.

A los compañeros de facultad quienes me animaron en los momentos difíciles, en especial Julián, Fede, Laura, Maria Eugenia y Celeste.

Al Comitee del Límite exterior de plataforma Argentina (COPLA), especialmente a Marcelo Paterlini y Daniel Abraham. 



\section{Índice general}

Prefacio $\quad$ V

Resumen vii

Abstract $\quad$ ix

Agradecimientos $\quad$ xi

1 Introducción $\quad 1$

1.1 Introducción . . . . . . . . . . . . . . . . . . . . . . 1

1.2 Contenidos . . . . . . . . . . . . . . . . . . 6

2 Márgenes continentales pasivos 9

2.1 Introducción . . . . . . . . . . . . . . . . . . . . 9 9

2.2 Algunos conceptos sobre la litósfera y los mecanismos de subsidencia . 11

2.3 Principales mecanismos de extensión . . . . . . . . . . . . . . 13

2.4 Evolución de un margen continental pasivo volcánico . . . . . . . . . . 14

2.5 Subsidencia en márgenes continentales pasivos . . . . . . . . . . . . 15

2.6 Margen continental argentino . . . . . . . . . . . . . . . . . 17

2.6.1 Clasificación del margen continental argentino . . . . . . . . . 18

2.6.2 Generalidades tectónicas del margen continental argentino y su conjugado el margen continental de Namibia . . . . . . . . . 20

2.6.3 Características tectónicas del margen continental argentino . . . 22

2.6.4 Anomalías magnéticas, gravimétricas y rasgos estructurales en el margen continental argentino . . . . . . . . . . 23

2.6.5 Un modelo de apertura del océano Atlántico . . . . . . . . . . . 24

2.6.6 Segmentación del margen continental argentino . . . . . . . . . 27

2.6.7 Arquitectura y estilo de las cuñas volcánicas del margen continental argentino . . . . . . . . . . . . . . . 30

3 Base de datos de gravedad 33

3.1 Bases de datos de gravedad consistente y documentada . . . . . . . . . . 33

3.2 Anomalías de gravedad derivadas de altimetría satelital . . . . . . . . . 34

3.3 Datos utilizados . . . . . . . . . . . . . . . . . 35

3.3.1 Grillas de anomalía de aire libre derivadas de altimetría satelital $\quad 35$

3.3.2 Datos de barco en el margen continental argentino . . . . . . . . 36

3.4 Comparación . . . . . . . . . . . . . . . . . . . . . . . . 39 
3.4.1 Comparación entre grillas de anomalías de gravedad derivadas de altimetría satelital . . . . . . . . . . . . . .

3.4.2 Comparación entre anomalías de gravedad derivadas de altimetría satelital y anomalías de gravedad marina . . . . . . . . . . . 41

3.5 Conclusiones del capítulo $3 \ldots \ldots$. . . . . . . . . . . . . 47

3.6 Propuesta para el análisis de cross-over . . . . . . . . . . . . . . 47

4 Anomalías de Bouguer y la expansión de Parker 49

4.1 Comparación de anomalías de Bouguer . . . . . . . . . . . . . 49

4.1.1 Método 1, con la expansión de Parker . . . . . . . . . . . 50

4.1.2 Método 2, anomalía de Bouguer completa . . . . . . . . . 51

4.1.3 Comparación entre ambos métodos . . . . . . . . . . . . 52

4.2 Conclusiones del Capítulo $4 \ldots \ldots \ldots$. . . . . . . . . . . . 59

5 Isostasia $\quad \mathbf{6 1}$

5.1 Conceptos generales . . . . . . . . . . . . . . . . . . . 61

5.2 Isostasia de Airy . . . . . . . . . . . . . . . . . . . . . . . 62

5.2.1 Cálculo del efecto gravimétrico con tres interfaces y compensación isostática local . . . . . . . . . . . . . . . . . . 62

5.2.2 Validación del método de cálculo de la anomalía gravimétrica utilizando interfaces . . . . . . . . . . . . . 65

5.3 Aplicación . . . . . . . . . . . . . . . . . . . 66

5.3.1 Espesor sedimentario . . . . . . . . . . . . . . 66

5.3 .2 Análisis de densidades . . . . . . . . . . . . . . . . . . 69

5.3.3 Topografía de las tres interfaces y sus contribuciones al efecto gravimétrico en la zona de estudio . . . . . . . . . . . . . 69

5.3.4 Anomalía isostática residual y la influencia de los sedimentos en su cálculo . . . . . . . . . . . . . . . . . . . 69

5.3.5 Deflexión del Moho necesaria para anular la anomalía isostática 70

5.3.6 Comparación de anomalías de aire libre . . . . . . . . . 76

5.3.7 Comparación con un perfil de refracción . . . . . . . . . . . 77

5.4 Isostasia Flexural . . . . . . . . . . . . . . . . . . . . . . . . . . . . 79

5.4.1 Aplicación de la teoría de flexión de placas a la litósfera de la Tierra ......................... 79

5.4 .2 Diagnóstico del ancho de banda de la flexura . . . . . . . . . . . . 80

5.5 Conclusiones del Capítulo $5 \ldots \ldots$. . . . . . . . . . . . . 83

6 Backstripping y Modelado de Gravedad Orientado por los Procesos 85

6.1 Cuencas de tipo rift . . . . . . . . . . . . . . 85

6.1.1 Backstripping ........................ 86

6.1.2 Subsidencia tectónica y Factor de estiramiento . . . . . . . . . . 91

6.1.3 Subplacado magmático . . . . . . . . . . . . . . . 96

6.2 Modelado de Gravedad Orientado por los Procesos . . . . . . . . . . . 98

6.3 Aplicación de las técnicas Backstripping y POGM . . . . . . . . . . 100

6.3.1 Aplicación 2D . . . . . . . . . . . . . . . . . . . . 102

6.3.2 Aplicación 3D . . . . . . . . . . . . . . . . . . . 107

6.4 Conclusiones del Capítulo 6 . . . . . . . . . . . . . . . . . 114 
7 Conclusiones generales $\quad 119$

A

125

A.1 Flexura de Placas en dos dimensiones . . . . . . . . . . . . . . 125

$\mathrm{B}$

B.1 Cálculo de anomalías de gravedad 2D . . . . . . . . . . . . . 131

C

C.1 Rutinas de GMT . . . . . . . . . . . . . . . . . . . 133

$\mathrm{D}$

135

D.1 Perfiles de la anomalía suma para las grillas de espesor Sedcombi y SedcombiCo . . . . . . . . . . . . . . . . . 135 



\section{Índice de figuras}

1.1 Área de estudio: se ha dibujado en un recuadro amarillo la zona de estudio que corresponde a las latitudes $38.5^{\circ} \mathrm{S}$ y $49.25^{\circ} \mathrm{S}$ y las longitudes $50^{\circ} \mathrm{W}$ y $64^{\circ} \mathrm{W}$ sobre anomalías de aire libre. SAM: Sudamérica, MEB: banco Maurice Edwing, SAAR: dorsal Sudamérica-Antártica, MAR: dorsal meso Atlántica, BTJ: punto triple de Bouvet. . . . . . . . . . . . . . . .

2.1 Distribución global de márgenes continentales volcánicos (rosa) y no volcánicos (azul). Modificado de Cuna [20]. Los márgenes volcánicos donde se han identificado secuencias de SDRs se han identificado con puntos naranjas. Las secciones encerradas por los rectángulos grises corresponden al margen estudiado en esta tesis (Argentino) y a su conjugado del lado Africano (Namibia) . . . . . . . . . . . . . . . .

2.2 Principales límites composicionales y reológicos. El límite composicional más importante está entre la corteza y el manto. Modificado de Vargas $[90,1] \ldots \ldots \ldots \ldots \ldots \ldots \ldots$

2.3 Perfil de resistencia de la litosfera y corteza oceánica. Extraído de Vargas

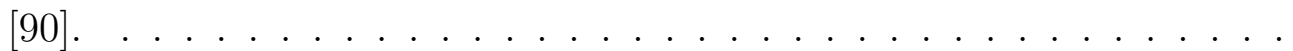

2.4 Mecanismo de extensión que genera subsidencia. (a) Extensión por tensión, (b) Subsidencia térmica. Extraído de Vargas [90] (c) Flexión por carga. Extraido de Watts [102]. . . . . . . . . . . . . . . .

2.5 Diferentes modelos de extensión litosférica para la formación de cuencas de rift y márgenes pasivos: a) cizalla pura: con una capa superior frágil sobre una capa inferior dúctil. El estiramiento dúctil puede venir acompañado de dilatación debida a la intrusión de materiales fundidos, b) cizalla simple: con un despegue de bajo ángulo que divide a la litosfera en una placa superior (hanging wall plate) y una placa inferior (footwall plate). El adelgazamiento de la litosfera inferior se transmite a lo largo del plano de despegue, produciendo una sección fuertemente asimétrica de la litosfera [90] y c) combinación de un modelo de estiramiento de la corteza de cizalla simple y pura. Modificado de Cuna [20] quien se basó en $[105,55] \ldots \ldots \ldots \ldots \ldots \ldots \ldots$

2.6 Diagrama de un margen volcánico idealizado. Modificado de Schmitd [82]. 15 
2.7 Modelo de emplazamiento de secuencias de reflectores buzantes que inclinan hacia el mar (SDRs). La evolución de los SDRs involucra domamiento e inyección de dique (1), sucesivos emplazamientos de flujo de lavas (1-4), los cuales luego del enfriamiento y carga con el flujo adicional sufren subsidencia (2-4) y se inclinan en la dirección hacia el mar. Modificado de Schmitd [82]. . . . . . . . . . . . . . . . .

2.8 Mapa con los diferentes tipos de márgenes: MCPV: margen continental pasivo volcánico, MCCi: margen continental cizallado, MCCo: margen continental combinado, 1: escarpa de Malvinas, 2: Golfo de San Jorge, 3: meseta de Malvinas. 4: banco Maurice Ewing, 5: zona de transferencia de Malvinas, 6: isla de los Estados, 7: banco de Burdwood, 8: dorsal norte de Scotia, 9: islas Georgias del Sur. . . . . . . . . . . . . .

2.9 Anomalías magnéticas, gravimétricas y rasgos estructurales en el margen continental argentino a) Anomalías magnéticas en relieve sombreado. b) Anomalías gravimétricas de aire libre derivadas de altimetría satelitaria [79]. El cuadro amarillo representa la región de estudio. Casi todas las líneas trazadas sobre las imágenes se encuentran en los dos mapas, a veces con diferente color para su contraste sobre el fondo. La anomalía magnética Tona y la discontinuidad del Colorado (DC) son de Ghidella et al. (1995) [35] y de Max et al. (1999) [58]. La línea negra cerca del borde de la plataforma es la isobata de $500 \mathrm{~m}$. En a) hay líneas negras que delimitan anomalías magnéticas positivas en el talud. Las mismas líneas están en blanco en b), el mapa de gravedad. Las cuñas volcánicas [45] están en ocre y en marrón. Notar que al norte de la DC la cuña y la anomalía positiva coinciden, pero no al sur. En azul en a) y rojo en b) se ha trazado la anomalía G de Rabinowitz y LaBrecque (1979) [76]. Las alineaciones mesozoicas están en gris en a) y en blanco en b), con algunas denominaciones. En el sector cercano a la cuña las anomalías van desde M10 a M4. Las líneas de rayas en color rosa son zonas de fractura interpretadas del mapa de gravedad. La línea de rayas que es verde en a) y blanca en b) es la interpretación de Ghidella et al. [36] del borde de la zona más influida por el arrastre de la transcurrencia en la zona de fractura de Malvinas (ZFM). En b) en color verde se han dibujado las estructuras digitalizadas de Ramos (1996) [78]. Modificado de Ghidella et al. [36]. . . . . . . . . . . . . . . . . . .

2.10 Ubicación de estructuras; las estructuras geológicas se digitalizaron de mapas en Ramos (1996) [78]; anomalías mesozoicas (serie M) según Cande et al. (1989)[14]; banda amarilla: cuña volcánica [45]. Línea segmentada negra: discontinuidad del Colorado. Modificado de la página web de Ghidella: http://www.martagh.com.ar/mararg/pictr2002/. . . . . . 
2.11 (a) Sudamérica rotada hacia África para la época de inicio de la apertura [36]. Para orientación se han dibujado las cuñas volcánicas que se encuentran a ambos lados del océano. También se han trazado las anomalías $\mathrm{G}$ de ambos lados. Las cuencas Colorado (CC), Valdés (CV) y Rawson (CR) tienen aproximadamente su configuración actual. DC: discontinuidad del Colorado. CO: cuenca de Outeniqua. También se ve la cuenca de Claromecó y los sistemas de Tandilia y Ventania. En África los plegamientos del El Cabo y la faja conductora Cabo Sur en la cuenca del Karoo. (b) Sudamérica rotada hacia áfrica para la época de la anomalía M4. Al sur de la DC no hay anomalías anteriores a M4 del lado sudamericano (M4 está en rojo y las anteriores en marrón). En líneas negras con relleno verde aparecen los contornos de los valores máximos de gravedad. La dirección de apertura es la del estiramiento de las cuencas (doble flecha en (a)). Modificado de Ghidella et al. [36] . . . . . . . . . .

2.12 Mapa estructural mostrando la distribución de manifestaciones volcánicas de gruesas cuñas de secuencias de reflectores que inclinan hacia el mar (SDRs), superficies volcánicas/magmáticas adicionales, depresiones del basamento oceánico y grabens pre-/syn-rift. Las zonas de transferencia y la segmentación del margen se interpretan a partir de variaciones en las características tectónico-volcánicas, distribución de sedimentos post-rift, se indican datos de campos potenciales y estudios previos [31].

2.13 Esquema que ilustra las principales superficies volcánicas-magmáticas en el margen continental argentino (no está a escala). Por debajo del talud hay uno o múltiples reflectores que inclinan hacia el mar (SDR) y un cuerpo de la corteza inferior de alta velocidad. Prominentes superficies hacia el mar son otros cuerpos de alta velocidad y las Reflexiones de la Corteza Superior (UCR, por sus siglas en inglés). El área más lejana de los SDRs está definida por la ocurrencia de altos exteriores y cuñas de SDRs antes de que comience la corteza oceánica. Modificado de Franke et al. [32]. . . . . . . . . . . . . . . . . . . . . . .

2.14 Perfil BGR98-05 situado en el segmento II del margen (Fig. 2.12). Múltiples SDRs están separadas por fuertes discontinuidades. OC: corteza oceánica, BU: discontinuidad de ruptura, PLe: equivalencia Pedro Luro, SDRs: reflectores intrabasamentales que inclinan hacia el mar. Modificado de $[31] \ldots \ldots \ldots \ldots \ldots \ldots \ldots$

3.1 Anomalía de aire libre versión V16.1 (a), izquierda y V18.1 (b), derecha. 36

3.2 Derrotas de barco de BGR98-1 (a), izquierda y BGR98-2 (b), derecha. . 38

3.3 Derrotas de barco de BGR87 (a), izquierda y BGI (b), derecha. . . . . 38

3.4 Diferencias entre grillas de anomalías de aire libre SyS18.1-SyS16.1 (a), izquierda y EGM2008-EGM96 (b), derecha. . . . . . . . . . . . . .

3.5 Diferencias entre grillas de anomalías de aire libre DNSC08-SyS16.1 (a), izquierda y DNSC08-SyS18.1 (b), derecha. . . . . . . . . . . . . .

3.6 Diferencias entre las grillas DTU10-SyS18.1 (a), izquierda y DNSC08DTU10 (b), derecha. . . . . . . . . . . . . . . . 
3.7 Diferencias entre anomalías de aire libre de (BGR87)-(DNSC08) (a), izquierda y (BGR98-1)-(DNSC08) (b), sobre mapa de batimetría, derecha. 43

3.8 Diferencias entre anomalías de aire libre de (BGR98-2)-(SyS18.1) (a), izquierda y (BGI)-(SyS18.1) (b), sobre mapa de batimetría, derecha. . .

3.9 Histograma de las diferencias BGR87-DNSC08 crudo (a), izquierda y editado (b), derecha. . . . . . . . . . . . . . . .

3.10 Histograma de las diferencias BGR87-SyS16.1 crudo (a), izquierda y editado (b), derecha. . . . . . . . . . . . . . . .

3.11 Histograma de las diferencias BGI-SyS16.1 crudo (a), izquierda y editado (b), derecha. . . . . . . . . . . . . . . . . .

3.12 Histograma de las diferencias BGI-SyS18.1 crudo (a), izquierda y editado (b), derecha. . . . . . . . . . . . . . . . .

4.1 Mapa de anomalía de Bouguer completa usando el programa (a) FA2BOUG y (b)gravfft hasta órden 4. . . . . . . . . . . . . . . 53

4.2 Diferencias (a) FA2BOUG y grdfft, (b)FA2BOUG y gravfft $\mathrm{n}=4 . \cdots \quad$. . 54

4.3 Diferencias (a) gravfft $\mathrm{n}=4$ y grdfft, (b) gravfft $\mathrm{n}=5$ y gravfft $\mathrm{n}=4 . \quad \ldots \quad 54$

4.4 Diferencias (a) FA2BOUG menos gravfft $\mathrm{n}=4$, (b) gravfft $\mathrm{n}=4$ menos grdfft. . . . . . . . . . . . . . . 56

4.5 Diferencias (a) FA2BOUG menos grdfft, (b) gravfft $\mathrm{n}=5$ menos grdfft. . 56

4.6 Diferencias FA2BOUG menos gravfft $\mathrm{n}=4$ en tres áreas longitudinales (a) $-63^{\circ} \leq \lambda \leq-60^{\circ}$, (b) $-60^{\circ} \leq \lambda \leq-56^{\circ}$, (c) $-56^{\circ} \leq \lambda \leq-51^{\circ}$ ( $\lambda$ es la longitud geográfica). . . . . . . . . . . . . . . . . . . 57

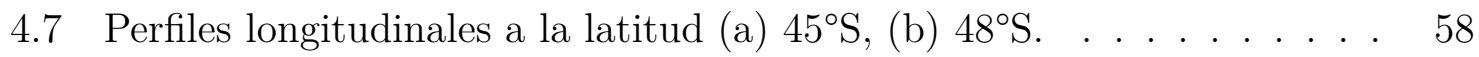

4.8 Cross plot de las diferencias Fullea-Parker1 (a), Parker4-Parker1 (b) y Fullea-Parker4 (c), vs la batimetría. . . . . . . . . . . . . 58

4.9 Diferencias Fullea-Parker4 $>5 \mathrm{mGal}$ (a),Parker4-Parker1 (b) > 5mGal. . 59

4.10 Diferencias Fullea-Parker1 $>5$ mGal. . . . . . . . . . . . . . . . 60

5.1 Interfaz agua-corteza ondulante respecto del valor medio de la batimetría. 63

5.2 Equilibrio isostático antes (descargado) y después de la carga de sedimentos (cargado). . . . . . . . . . . . . . . . .

5.3 Espesor sedimentario. Se ha graficado la isobata de $500 \mathrm{~m}$, en color negro, para referencia. 1: cuenca del Colorado, 2: cuenca de Valdés, 3: cuenca de Rawson, 4: cuenca Argentina. . . . . . . . . . . . . .

5.4 (a) Topografía (Batimetría) y (b) Contribución del efecto gravimétrico de la interfaz agua-sedimentos. . . . . . . . . . . . . . . .

5.5 (a) Topografía (Profundidad del basamento) y (b) Contribución del efecto gravimétrico de la interfaz sedimentos-corteza. . . . . . . . . . . . .

5.6 (a) Topografía (Moho) y (b) Contribución del efecto gravimétrico de la interfaz corteza-manto. . . . . . . . . . . . . . .

5.7 Anomalía de aire libre (a) arriba, anomalía isostática Parker $\mathrm{n}=4$ (b) abajo (izquierda) y diferencia de anomalía isostática Parker $\mathrm{n}=4$ sin sedimentos y con sedimentos (c) abajo (derecha). En las tres figuras se ha dibujado la isobata de $500 \mathrm{~m}$, en color negro, para referencia. . . . . 
5.8 Relieve del Moho calculado de tres formas diferentes: que compensa la anomalía isostática (ver texto) (a) Mohofin1 y (b) Mohofin2 y el que se obtiene al invertir la anomalía de Bouguer (c) Moho3Dinv. . . . . . . .

5.9 Relieve del Moho que compensa la anomalía isostática, calculado de tres formas diferentes a distintas latitudes (ver texto) -41.77 (arriba, izquierda)(a) -42.77 (arriba, derecha) (b). -43.52 (abajo, izquierda) (c). -44.02 (abajo, derecha) (d). . . . . . . . . . . . .

5.10 Perfiles a $42^{\circ} \mathrm{S}(\mathrm{a}), 42.5^{\circ} \mathrm{S}(\mathrm{b}), 43^{\circ} \mathrm{S}$ (c) y $43.5^{\circ} \mathrm{S}$ (d) de latitud, de la anomalía de gravedad de aire libre observada (gob) y estimada con 3 interfaces (wssccm1(grdfft), wssccm4 (gravfft) y 3Dinv(gestim3D). Notar que wssccm1 y wssccm4 fueron calculadas con el método de tres interfaces usando un Moho obtenido por compensación hidrostática de masas, mientras que gestim3D fue calculada de un Moho derivado de invertir la anomalía de Bouguer. Esto explicaría las diferencias grandes que se observan por ejemplo entre 300 y $400 \mathrm{~km}$ para el perfil a los -42 y entre 180 y $300 \mathrm{~km}$ para el perfil a los -43. . . . . . . . . . . . . . . .

5.11 Perfiles a $42^{\circ} \mathrm{S}(\mathrm{a}), 42.5^{\circ} \mathrm{S}(\mathrm{b}), 43^{\circ} \mathrm{S}$ (c) y $43.5^{\circ} \mathrm{S}$ (d) de latitud, de la anomalía isostática calculada a partir del Mohofin1 (AIfin1), Mohofin2 (AIfin2) y a partir del Moho3Dinv (isoestim). . . . . . . . . . . .

5.12 Perfil EO de refracción de la campaña BGR98 a la latitud -43.5 [30], interfaces digitalizadas (batyFranke, Mohod-Franke, MohouFranke. . .

5.13 Perfiles longitudinales $43^{\circ} \mathrm{S}$ digitalizado de Franke y de las interfaces profb1: profundidad del basamento, batyS\&S: batimetría [79], Moho1 (sección 5.3.4 1)), Mohofin2 (sección 5.3.4 2)), Moho 3D (sección 5.3.4 3)). Las líneas Mohou-Franke y Mohod-Franke encierran la capa de alta velocidad (AV+,aproximadamente entre los $350 \mathrm{~km}$ y los $550 \mathrm{~km}$ ). . . .

5.14 Modelo para el cálculo de la fuerza restitutiva en las placas litosféricas deflectadas por una carga aplicada $q_{a}$ para el caso oceánico (Turcotte \&

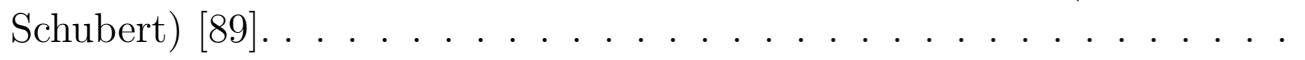

5.15 Función de respuesta flexural $\phi(k)$, modificado de Watts 2001 [103]. . .

6.1 Los principios del backstripping tipo Airy, modificado de Cuna [20]. . .

6.2 Backstripping multicapa. En a) la primera columna representa lo observado (al presente) totalmente cargado por una secuencia de 3 unidades. Al Tiempo1', la Unidad 1 es descompactada a su posición original y su densidad (ec. 6.1 y 6.2). Al Tiempo 1 se restablece isostáticamente la profundidad del basamento en ausencia de la Unidad 1 (backstripping, ec. 6.5), recobrando la subsidencia Y1 al tiempo paso 1. Al Tiempo 2' el espesor de la Unidad 2 es decompactado y la Unidad 1 es compactada de acuerdo a la nueva profundidad. Al Tiempo 2 la Unidad 2 es descompactada totalmente y la Unidad 1 es compactada parcialmente, luego se aplica el backstripping recobrando la subsidencia Y2 al tiempo 2. Este procedimiento es repetido para la capa 3. En b) se dibuja la correspondiente curva de subsidencia Y, modificado de Cuna [20]. . . . 
6.3 Los principios del backstripping flexural. La región sombreada muestra la cuña sedimentaria. La línea de guiones intermitentes representa la posición que tendría el basamento en ausencia de la carga de sedimentos. Modificado de Watts (2001) [103] . . . . . . . . . . . . . . . . . .

6.4 Dos etapas del modelo propuesto por McKenzie [59] para explicar la formación y evolución de cuencas de rift: subsidencia (izquierda) y estructura termal de la litosfera (derecha) a) litosfera pre estiramiento. b) Despúes de un evento de rift instantáneo. c) Después de la relajación termal de la litosfera y recupero del gradiente de la temperatura inicial. $T_{c}$ espesor inicial de corteza, L espesor de la litosfera, $t_{m}$ es la temperatura de la base de la litosfera, $S_{i}$ es la subsidencia inicial de la cuenca, $S_{f}$ es la subsidencia final de la cuenca, $\beta$ es el factor de estiramiento, TTS subsidencia tectónica total, $\rho_{m}$ y $\rho_{c}$ son valores promedio de densidades del manto y de la corteza previos e inmediatamente posteriores al estiramiento, $\rho_{m}^{\prime} \mathrm{y} \rho_{c}^{\prime}$ son valores promedio de densidades del manto y de la corteza posteriores al estiramiento cuando se han alcanzado condiciones

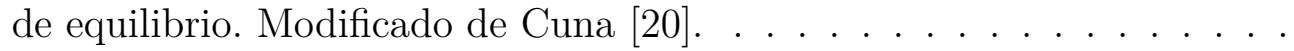

6.5 Relación entre el estiramiento y el espesor de la corteza. . . . . . . . . .

6.6 Modelo simple de subplacado magmático de la corteza de espesor uniforme. Modificado de Watts [103]. . . . . . . . . . . . . . .

6.7 Diagrama de flujo para aplicar el POGM adaptado de Cuna [20] y Stewart et al. [86].

6.8 Modelado de Gravedad Orientado por los Procesos: diagrama sintético. Modificado de Cuna [20]. . . . . . . . . . . . . . . .

6.9 Backtripping para el caso perfil $43^{\circ} \mathrm{S}$ y un $T_{e}=10 \mathrm{~km}$. baty: batimetría, pbaty: paleobatimetría y basa: basamento. . . . . . . . . . . . . . 102

6.10 Factor de estiramiento $\beta$ para el caso perfil $43^{\circ} \mathrm{S}$. . . . . . . . . . . 103

6.11 Anomalía del rift para el caso perfil $43^{\circ}$ S. . . . . . . . . . . . . . . . . . 104

6.12 Carga de los sedimentos para el caso perfil $43^{\circ} \mathrm{S}$ y $T_{e}=10 \mathrm{~km}$, donde las líneas verticales verdes representan la altura de carga. baty: batimetría, pbaty: paleobatimetría y basa:basamento (arriba), moho-flex: Moho flexionado (abajo). Las líneas verdes verticales representan la altura de carga. . . . . . . . . . . . . . . . .

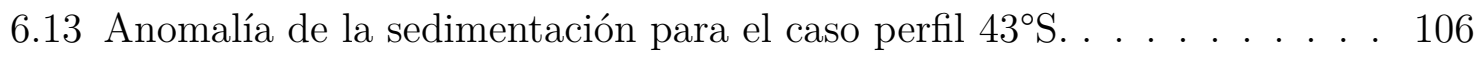

6.14 Anomalía suma para el caso perfil 43ㅇ․ . . . . . . . . . . . . . 107

6.15 Anomalía suma para el caso perfil $43^{\circ} \mathrm{S}$ calculada para las grillas de espesor sedimentario Sedcombi (a) y SedcombiCO (b).

6.16 Una estimación 3D de la paleobatimetría al tiempo del rift (a) y del paleoMoho (b), a partir de perfiles 2D. CC: cuenca del Colorado, CV: cuenca de Valdés y CR: cuenca de Rawson. . . . . . . . . . . . . . .

6.17 factor de estiramiento $\beta$ en a) enfatizando la zona de plataforma en b) la zona marina profunda. En ambas figuras se ha graficado en color negro la isobata de $500 \mathrm{~m}$ para referencia. . . . . . . . . . . . . . . 110

6.18 Anomalía del rift desde 2D (a) y desde 3D (b). En ambas figuras se ha graficado en color negro la isobata de $500 \mathrm{~m}$ para referencia. . . . . . . 111 
6.19 Flexura del basamento y del Moho. Se ha graficado en color negro la isobata de $500 \mathrm{~m}$ para referencia. . . . . . . . . . . . .

6.20 Anomalía de la sedimentación desde 2D (a) y desde 3D (b). En ambas figuras se ha graficado en color negro la isobata de $500 \mathrm{~m}$ para referencia.112

6.21 Anomalía suma desde 2D (a) y desde 3D (b). En ambas figuras se ha graficado en color negro la isobata de $500 \mathrm{~m}$ para referencia. . . . . . .

6.22 Anomalía isostática según Airy (a) y la calculada con POGM para $T_{e}=$ $15 \mathrm{~km}$ (b). En ambas figuras se ha graficado en color negro la isobata de $500 \mathrm{~m}$ para referencia. . . . . . . . . . . . . . . .

6.23 Anomalía del rift desde 2D, se han superpuesto las estructuras del mapa de segmentación del margen. Líneas marrón oscura representan pre-/syn rift grabens, líneas negras zonas de transferencia, líneas fucsia intrusivos, líneas verde claro son cuñas del mismo episodio 1, líneas marrón claro son cuñas del mismo episodio 2, líneas azul son depresiones del basamento oceánico y líneas amarillo son flujos de lavas basálticos. . . . . . . . . .

6.24 Anomalía suma desde 2D, se han superpuesto las estructuras del mapa de segmentación del margen. Líneas marrón oscura representan pre-/syn rift grabens, líneas negras zonas de transferencia, líneas fucsia intrusivos, líneas verde claro son cuñas del mismo episodio 1, líneas marrón claro son cuñas del mismo episodio 2, líneas azul son depresiones del basamento oceánico y líneas amarillo son flujos de lavas basálticos. . . . . . . . . .

6.25 Anomalía isostática según Airy (a) y la calculada con POGM para $T_{e}=15 \mathrm{~km}$, se han superpuesto las estructuras de el mapa de segmentación del margen. Líneas negras representan pre-/syn rift grabens, líneas blancas zonas de transferencia, líneas fucsia intrusivos, líneas marrón oscuro son cuñas del mismo episodio 1, líneas azul son cuñas del mismo episodio 2, líneas verde claro son depresiones del basamento oceánico y líneas amarillo son flujos de lavas basálticos.

A.1 Fuerzas y torques en una sección pequeña de una placa flexionada, http://www.colorado.edu/geolsci/courses/GEOL5690/FlexureNotes.pdf (Turcotte \& Schubert) [89]. . . . . . . . . . . . . . . . . .

A.2 Esfuerzo normal en una sección transversal de una placa elástica curvada (Turcotte \& Schubert) [89]. . . . . . . . . . . . . . . . . .

A.3 Extensión y contracción longitudinal a una distancia $z$ desde la mitad del plano de la placa (Turcotte \& Schubert) [89]. . . . . . . . . . . . .

A.4 Esquema ilustrativo de la relación geométrica de la placa flexionada (Turcotte \& Schubert) [89]. . . . . . . . . . . . . . . . . .

B.1 Aproximación de un cuerpo bidimensional por un polígono de $\mathrm{N}$ lados, (modificado de $[9]) \ldots \ldots \ldots \ldots \ldots$

D.1 Anomalía suma para el caso del perfil $39^{\circ}$ S calculada para las grillas de espesor sedimentario Sedcombi (a) y SedcombiCo (b) . . . . . . . . . . 136

D.2 Anomalía suma para el caso del perfil $40^{\circ} \mathrm{S}$ calculada para las grillas de espesor sedimentario Sedcombi (a) y SedcombiCo (b) . . . . . . . . . 136 
D.3 Anomalía suma para el caso del perfil 41ํㅗ calculada para las grillas de espesor sedimentario Sedcombi (a) y SedcombiCo (b). . . . . . . . . . . 137

D.4 Anomalía suma para el caso del perfil $42^{\circ} \mathrm{S}$ calculada para las grillas de espesor sedimentario Sedcombi (a) y SedcombiCo (b) . . . . . . . . . . . 137

D.5 Anomalía suma para el caso del perfil $43^{\circ} \mathrm{S}$ calculada para las grillas de espesor sedimentario Sedcombi (a) y SedcombiCo (b) . . . . . . . . . . . 138

D.6 Anomalía suma para el caso del perfil $44^{\circ} \mathrm{S}$ calculada para las grillas de espesor sedimentario Sedcombi (a) y SedcombiCo (b). . . . . . . . . . . 138

D.7 Anomalía suma para el caso del perfil $45^{\circ} \mathrm{S}$ calculada para las grillas de espesor sedimentario Sedcombi (a) y SedcombiCo (b). . . . . . . . . . . 139

D.8 Anomalía suma para el caso del perfil $46^{\circ} \mathrm{S}$ calculada para las grillas de espesor sedimentario Sedcombi (a) y SedcombiCo (b). . . . . . . . . . . 139

D.9 Anomalía suma para el caso del perfil $47^{\circ} \mathrm{S}$ calculada para las grillas de espesor sedimentario Sedcombi (a) y SedcombiCo (b). . . . . . . . . . . 140

D.10 Anomalía suma para el caso del perfil $48^{\circ} \mathrm{S}$ calculada para las grillas de espesor sedimentario Sedcombi (a) y SedcombiCo (b). . . . . . . . . . . 140 


\section{Índice de tablas}

3.1 Estadística de las anomalías de gravedad derivadas de altimetría. Uni-

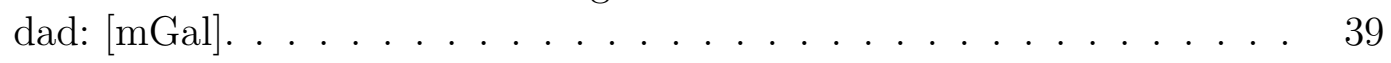

3.2 Estadística de diferencias entre grillas de anomalías de gravedad deriva-

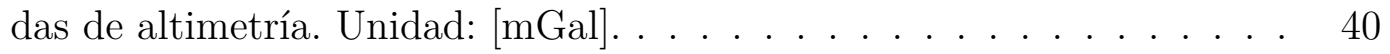

3.3 Estadística de diferencias entre anomalías de aire libre de la campaña

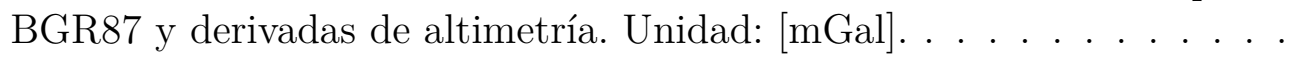

3.4 Estadística de diferencias entre anomalías de aire libre de la campaña BGR98-1 y derivadas de altimetría. Unidad: [mGal]. . . . . . . . . . . .

3.5 Estadística de diferencias entre anomalías de aire libre de la campaña BGR98-2 y derivadas de altimetría. Unidad: [mGal]. . . . . . . . . . .

3.6 Estadística de diferencias entre anomalías de aire libre de la campaña BGI y derivadas de altimetría. Unidad: [mGal]. . . . . . . . . . . . . 43

3.7 Estadística de diferencias entre anomalías de aire libre de la campaña BGR87 editada y derivadas de altimetría. Unidad: [mGal]. . . . . . . . 44

3.8 Estadística de diferencias entre anomalías de aire libre de la campaña BGR98-1 editada y derivadas de altimetría. Unidad: [mGal]. . . . . . . 44

3.9 Estadística de diferencias entre anomalías de aire libre de la campaña BGR98-2 editada y derivadas de altimetría. Unidad: [mGal]. . . . . . . 44

3.10 Estadística de diferencias entre anomalías de aire libre de la campaña BGI editada y derivadas de altimetría. Unidad: $[\mathrm{mGal}]$. . . . . . . . . .

4.1 Estadística de diferencias de anomalías de Bouguer calculadas con gravfft, grdfft, FA2BOUG y su comparación. Unidad: [mGal]. . . . . . . . . . .

6.1 rms del POGM para perfiles con diferentes valores de $T_{e}$ y a diferentes latitudes para dos datos de espesor sedimentario: Sedcombi y SedcombiCO108

6.2 rms del POGM para grillas con diferentes valores de $T_{e}$ para las estimaciones: 3D y 3D a partir de 2D . . . . . . . . . . . . . . 113 



\section{Capítulo 1}

\section{Introducción}

\section{$1.1 \quad$ Introducción}

En esta tesis abordamos el estudio del margen continental argentino en el sector volcánico pasivo, mediante un método potencial, el método gravimétrico.

\section{Los márgenes continentales}

Los márgenes continentales son dominios fisiográficos contenedores de espesas secuencias sedimentarias, considerados como zonas de transición entre las cuencas oceánicas y los continentes [92]. Los márgenes continentales pasivos se clasifican como volcánicos o no volcánicos de acuerdo a la cantidad de vulcanismo que haya ocurrido durante el rift. Los márgenes volcánicos están caracterizados por secuencias de reflectores que inclinan hacia el mar (SDRs, por sus siglas en inglés: Seaward Dipping Reflectors ), formados por la erupción y subsecuente subsidencia de flujo volcánico ocurrida durante el rift, y por capas de corteza inferior con velocidades sísmicas de onda $\mathrm{P}$ anormalmente altas y altas densidades. Las capas de corteza inferior con velocidades altas debajo del talud continental varían de entre 5 a $15 \mathrm{~km}$. Tanto las capas de la corteza inferior como los complejos SDRs están asociados espacialmente con la transición océano-continente. Generalmente suele denominarse subplacado magmático a capas de la corteza inferior, que pueden estar compuestas de material derivado del manto debajo de la corteza o material ígneo adicionado entre o debajo de la corteza, aunque el término subplacado magmático no es del todo apropiado considerando que corteza vieja no está siendo subplacada y podría ser parte de una corteza oceánica inicial. La naturaleza y localización del límite entre la corteza continental y oceánica es de fundamental importancia para la comprensión de los procesos que tiene lugar en un rift [84]. El efecto de borde de la anomalía gravimétrica de aire libre asociado a los márgenes continentales de tipo pasivo es una de las características distintivas de la gravedad en zonas marinas. Este efecto se debe en gran parte a la transición entre corteza continental y oceánica, por sus diferentes espesores. Aunque el adelgazamiento de la corteza es una de las principales contribuciones a este efecto, actualmente se reconoce que procesos tales como extrusión basáltica, el subplacado magmático y la carga sedimentaria pueden modificarlo considerablemente $[102,86]$. En una forma simple la anomalía contiene un "alto" que se correlaciona con la parte exterior de la plataforma y un "bajo" asociado con la 
región del talud. Algunos márgenes tienen un bajo tierra adentro en el alto y un alto mar adentro en el bajo [102]. El efecto de borde es muy sensible a la localización de la transición, cambiando abruptamente si se desplaza algunos kilómetros tierra adentro o mar adentro. Dado que la zona de transición es producto del rifting, éste tiene el potencial de decirnos algo acerca del estilo y la geometría de la dinámica de la ruptura de los continentes.

La transición océano-continente en un margen volcánico ocurre sobre una distancia relativamente corta de $50-80 \mathrm{~km}$ a $150 \mathrm{~km}$ comparado con un margen no volcánico. La transición más abrupta en un margen volcánico se atribuye a debilidad de la litosfera debida a grandes cantidades de roca ígnea intruída [84].

\section{El margen argentino}

El margen continental pasivo del Atlántico Sur se formó como resultado de la apertura de este océano debido a la separación de África de Sudamérica durante el Cretácico temprano [76]. La apertura del fondo oceánico comenzó en la parte sur del Atlántico Sur y se propagó hacia el norte. La ruptura del continente con la subsecuente expansión del fondo oceánico ha sido acompañada por volcanismo de gran escala que produjo las provincias basálticas de Paraná-Etendeka [84].

El margen continental argentino constituye uno de los más extensos del mundo. Su mayor desarrollo, entre los paralelos $35^{\circ} \mathrm{S}$ y $48^{\circ} \mathrm{S}$, corresponde a un margen pasivo de tipo Atlántico clasificado como Margen Continental Extensional Volcánico. Los procesos sedimentarios erosivos y depositacionales han sido muy activos, con desarrollo de importantes sistemas de cañones submarinos (transversales al talud), condicionados por una activa dinámica de la masas de agua de origen antártico favoreciendo la formación de grandes acumulaciones de sedimentos (longitudinales al talud), conformando uno de los sistemas depositacionales contorníticos más extensos del mundo [92].

El sector del margen argentino bajo estudio en esta tesis está comprendido entre los $38.5^{\circ} \mathrm{S}$ y los $49.25^{\circ} \mathrm{S}$ (plataforma de Malvinas) de latitud y las longitudes $50^{\circ} \mathrm{W}$ y $64^{\circ} \mathrm{W}$ (Fig. 1.1). Las características volcánicas quedan evidenciadas por la presencia de secuencias de cuñas de reflectores sísmicos intra-basamentales que inclinan hacia el mar (SDRs).

Estudios previos de anomalías magnéticas y de gravedad del área han permitido detectar una discontinuidad estructural llamada discontinuidad del Colorado, que atraviesa la plataforma continental y el talud continuándose en la corteza oceánica, hasta la anomalía magnética Mesozoica M2. La anomalía magnética G, que se observa también en el margen africano, tiene un notable desplazamiento mar adentro al atravesar la discontinuidad del Colorado. También se observa un cambio grande en las características volcánicas $[35,31]$.

\section{La anomalía de gravedad y la isostasia}

La anomalía de gravedad de un margen continental puede considerarse como el resultado de todos los procesos que tienen lugar a través del tiempo [96]. Estos incluyen rifting, sedimentación y subplacado magmático. El análisis de la isostasia es de fundamental 


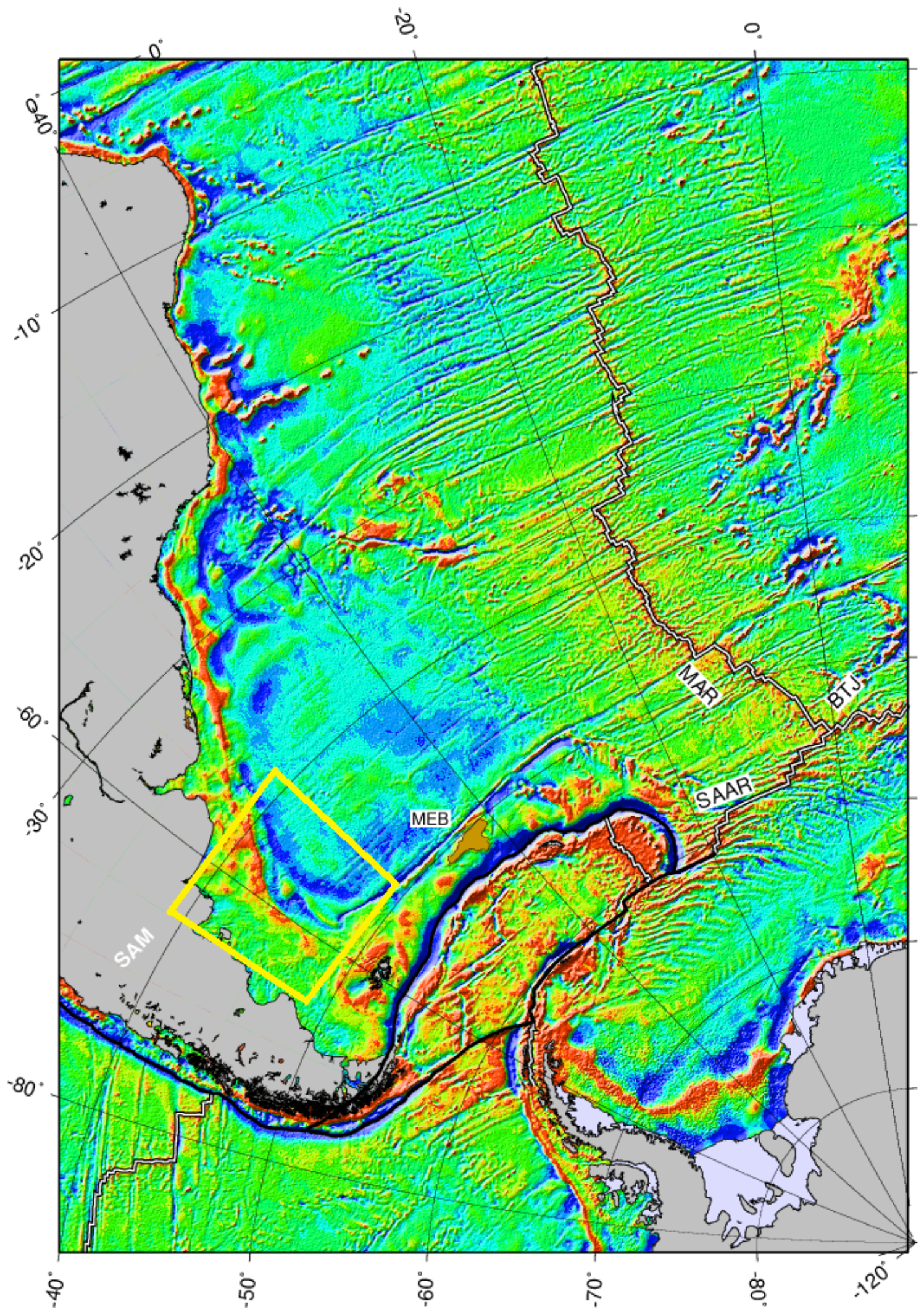

Figura 1.1. Área de estudio: se ha dibujado en un recuadro amarillo la zona de estudio que corresponde a las latitudes $38.5^{\circ} \mathrm{S}$ y $49.25^{\circ} \mathrm{S}$ y las longitudes $50^{\circ} \mathrm{W}$ y $64^{\circ} \mathrm{W}$ sobre anomalías de aire libre. SAM: Sudamérica, MEB: banco Maurice Edwing, SAAR: dorsal Sudamérica-Antártica, MAR: dorsal meso Atlántica, BTJ: punto triple de Bouvet. 
importancia para la interpretación gravimétrica, dado que trata del equilibrio y compensación de las grandes masas que componen el planeta. Este análisis presenta cierta dificultad en los márgenes continentales, y por ese motivo requiere que se le asigne una dedicación especial en estos estudios. El concepto de isostasia describe la manera en que las superficies topográficas en la superficie de la tierra son compensadas en profundidad. Los dos modelos más comúnmente usados fueron desarrollados por Airy(1885) y Pratt(1885). En el modelo de Airy se supone que la corteza tiene densidad constante pero es más gruesa en zonas montañosas que en tierras bajas. Para el modelo de Pratt, la densidad promedio de la corteza es menor en zonas montañosas que en tierras bajas. Estudios sísmicos muestran que el modelo de Airy explica satisfactoriamente variaciones de la estructura de la corteza asociadas con superficies como zonas montañosas y márgenes continentales.

El modelo de Airy, sin embargo, implica que la corteza no puede soportar esfuerzos de cizalla verticales. Barrel (ver en [103] un resumen) argumentó en los inicios del 1914 que el equilibrio isostático planteado por Airy no podría existir en toda la superficie de la Tierra debido al esfuerzo finito de la corteza. Barrel (1914) definió a esta capa rígida superior como la litosfera de la Tierra.

Un número de estudios han investigado la respuesta de la litosfera a cargas como montañas e islas oceánicas. Estos estudios indican que en un número de casos, la respuesta de la litosfera a cargas superficiales puede modelarse como una placa delgada que yace sobre un fluido. Este modelo de isostasia, referido como modelo flexural, es similar al de Airy, en el que las cargas son soportadas por variación del espesor de la corteza, pero difiere en que incluye esfuerzos laterales de la corteza. Un parámetro utilizado en el modelo flexural, que caracteriza la respuesta de la litosfera a estas cargas es la rigidez flexural efectiva [49].

\section{El backstripping}

Un camino para entender mejor el rol relativo de los procesos que ocurren en un margen, como el importante proceso de la carga sedimentaria, es el backstripping [96, 85]. Ésta es una técnica cuantitativa que puede utilizarse para remover los efectos de carga de esos procesos en orden de aislar la subsidencia tectónica (o aun levantamiento) que el margen habría experimentado en su ausencia. El método backstripping, sin embargo, requiere del conocimiento de la forma en que la litosfera responde a las cargas verticales asociadas con el rifting, sedimentación y subplacado.

\section{El método flexural y el Modelado de Gravedad Orientado por los Procesos}

Un camino útil para modelar la respuesta de la litosfera a cargas a tiempos de escala geológica ha sido el uso de un modelo de placa elástica delgada. Para lograr un mejor entendimiento de las causas del efecto de borde del margen se ha diseñado un método que sigue paso a paso los diferentes procesos ocurridos en el margen, como sedimentación y subplacado magmático, calculando la anomalía de cada paso. Es el método de gravedad Orientado por los Procesos (POGM) [102, 103], que hemos adoptado considerando que tiene grandes ventajas respecto a un modelado estático para 
aproximarnos a la interpretación de anomalías de gravedad. En un modelado estático, la estructura de densidades determina lo que mejor explica a las anomalías de gravedad. Tal aproximación, ha sido útil para determinar las propiedades físicas de la corteza y el manto en regiones continentales. Sin embargo nos ha aportado poco sobre los roles que cumplen los procesos de sedimentación y subplacado magmático junto a los efectos de carga que participan en la evolución del margen. De estudios de flexura conocemos las grandes diferencias que existen entre las estructuras de espesores elásticos $\left(T_{e}\right)$ de las litosferas oceánica y continental. En los océanos, $T_{e}$ está dado aproximadamente por la profundidad de la isoterma de $450^{\circ} \mathrm{C}$, resultado basado en modelos de enfriamiento de placas. Por ejemplo, $T_{e}$ aumenta de 4 a $12 \mathrm{~km}$ en las dorsales meso oceánicas, donde la litosfera es relativamente joven y caliente a más de $30 \mathrm{~km}$ donde la litosfera es antigua y fría. Estudios en los continentes, sin embargo, no muestran una relación simple entre $T_{e}$ y la edad termal. En general, de todos modos, pensamos en zonas débiles (bajos $T_{e}$ ) como zonas típicas de rifts, mientras que zonas rígidas (altos $T_{e}$ ) parecen caracterizar cratones.

Resultados de estudios de flexura sugieren que podemos determinar la estructura de $T_{e}$ del margen. Podemos determinar si los sedimentos se han depositado sobre corteza continental u oceánica. Por ejemplo, si el efecto de borde de la anomalía de gravedad observado se explica por un modelo de carga de sedimentos de la litosfera que fue inicialmente débil y luego se hizo más rígida con el tiempo, esto está de acuerdo con que los sedimentos fueron depositados en una corteza oceánica más que en una continental. Si de otro modo la anomalía se explica mejor por un modelo en el cual los sedimentos fueron depositados en una litósfera que se mantuvo débil durante y después del rift, esto sugiere de que los sedimentos se depositaran durante un rift de corteza continental [102].

\section{Antecedentes}

Con anterioridad a la propuesta de la presente tesis, la directora ha trabajado en el estudio del margen argentino. Esto fue en colaboración con colegas del Servicio de Hidrografía Naval y del Instituto de Física de Rosario con quienes ha llevado a cabo varios estudios en la década 2000 - 2010, muchos de los cuales se citan en el trabajo de tesis; casi simultáneamente tuvieron lugar los estudios intensivos llevados a cabo por la Comisión para la delimitación del límite externo de la plataforma continental argentina (COPLA), así como también por misiones marinas sobre todo alemanas del BGR (Servicio Geológico Alemán), de manera que en los últimos 15 años se han obtenido importantes progresos en el conocimiento del margen y se ha recopilado un interesante cantidad de datos sísmicos, batimétricos, magnéticos y gravimétricos, y existen una buena cantidad de trabajos publicados que muestran el progreso de dichos estudios. Durante todos estos años la directora ha notado la necesidad de aplicar modelos flexurales a los datos de gravedad para modelado y estudio de la isostasia, estudio que considera de suma importancia, y no se dio la oportunidad hasta el momento de planificar la presente tesis.

Esta tesis apunta a investigar un esquema de compensación isostática del campo de gravedad en 3D a través de tres interfaces, con el método de Airy, el método flexural y el Modelado de Gravedad Orientado por los Procesos (POGM, por sus siglas en inglés) 
que integra las técnicas de backstripping y modelado de gravedad con el objetivo de caracterizar el margen continental argentino de tipo pasivo volcánico a través del uso de datos de gravedad.

\subsection{Contenidos}

Los objetivos generales del trabajo de tesis propuesto consisten en:

- Caracterizar el margen volcánico pasivo con datos de anomalías gravimétricas.

- Analizar la compensación isostática a través de estudiar y/o desarrollar programas para calcular la anomalía isostática en una amplia región en el mar argentino y zona oceánica adyacente con especial énfasis en los métodos de Airy y flexural.

Los objetivos específicos de las tareas de investigación propuestas pueden sintetizarse del siguiente modo:

- Organizar bases de datos gravimétricos, topográficos batimétricos y de espesor sedimentario en una amplia región del margen argentino y zona oceánica adyacente.

- Calcular la anomalía isostática de gravedad en un esquema de tres interfaces mediante los métodos de Airy y flexural, documentando las suposiciones involucradas y parámetros usados.

- Comparar resultados obtenidos con la hipótesis de compensación local con los de la flexural.

- Identificar un juego de parámetros (espesor elástico equivalente, densidades, etc.), que minimice la anomalía isostática obtenida.

- Comparar los aportes gravimétricos con los magnéticos y sísmicos.

En el Capítulo 2 se introducen las principales características geológicas de los márgenes pasivos.

En el Capítulo 3 se emprende la tarea de organizar datos de anomalías gravimétricas de aire libre en la región y compilar los datos en una base de datos consistente y documentada. Para ello se compararon grillas de anomalías de gravedad de aire libre derivadas de misiones de altimetría satelital con anomalías de gravedad marina calculadas a partir de datos de cruceros en el margen argentino y zona oceánica adyacente. Se analizaron los datos de barco de distintas campañas realizadas en épocas diferentes y en una primera etapa se organizaron en una base de datos común, a partir de la cual se crearán versiones mejoradas con nuevas correcciones.

En el Capítulo 4 se focaliza en comparar el cálculo de la anomalía de Bouguer con dos métodos. El primer método utiliza la relación entre topografía y gravedad con el desarrollo de Parker en el dominio de Fourier y el segundo método calcula la anomalía de Bouguer completa (Bullard A, B o C) en varios dominios espaciales con diferentes aproximaciones para la atracción de prismas rectangulares y cónicos en las cercanías de la estación [34]. El propósito de este análisis fue validar una metodología de cálculo 
para la anomalía isostática utilizando transformada de Fourier, en una amplia región del mar argentino con el esquema de Airy.

En el Capítulo 5 se investiga el mecanismo de compensación de tipo Airy con un esquema de tres interfaces utilizando las metodologías que se describen a continuación: 1) en sentido directo con diferentes aproximaciones del desarrollo de Parker (utilizando los programas grdfft [106] y gravfft [57]) a través del cálculo de 3 interfaces con compensación isostática local.

2) invirtiendo la anomalía obtenida en 1), determinando la deflexión del Moho necesaria para compensarla (anularla) utilizando el mismo esquema de tres interfaces y el método iterativo de Parker-Oldenburg [67] con más términos en la inversión, a través del programa 3Dinv.m [39]. La interfaz corteza-manto (Moho) así calculada representa una superficie más realista que la calculada con un término en la inversión y que la estimada con datos de topografía y espesor sedimentario, pero no deja de ser un ensayo para probar los métodos numéricos.

Si bien el experimento constituye una suposición esquemática para probar los métodos numéricos, encontramos que en la comparación con el único perfil de refracción digitalizado disponible, la interfaz "Moho" invertida reproduce bastante bien el Moho modelado en dicho perfil, para el caso del método iterativo.

En el Capítulo 6 se focaliza en la aplicación de las técnicas backstripping y Modelado de Gravedad Orientado por los Procesos. El backstripping es una técnica cuantitativa basada en la remoción de la carga desde el basamento, aplicada bajo las hipótesis de isostasia de Airy o flexural, asumiendo conocida la carga de la corteza producida por el agua y la de los sedimentos, lo que supone que conocemos el espesor sedimentario. La grilla de espesor sedimentario utilizada ha sido compilada de varias fuentes de datos, resultado de digitalizaciones de mapas publicados y tiempo de ida y vuelta de la señal sísmica (TWT), con una densidad constante y no incorpora datos de estratigrafía de pozos. A partir del backstripping [103] es posible determinar la subsidencia tectónica directamente desde datos estratigráficos. 



\section{Capítulo 2}

\section{Márgenes continentales pasivos}

En el presente Capítulo hicimos una revisión integral de las características particulares de los márgenes continentales pasivos especialmente aquellos de origen volcánico. Analizamos las características del margen continental argentino en cuanto a morfología, origen tectónico y aspectos particulares que lo distinguen como anomalías magnéticas y gravimétricas, segmentación y cuñas volcánicas y otros aspectos geográficos, geológicos y geofísicos que son de importancia para dar un marco tanto a estudios posteriores como a los Capítulos que siguen en la tesis.

\section{$2.1 \quad$ Introducción}

Los márgenes continentales pasivos están asociados con rifts continentales y la subsecuente formación de cuencas oceánicas. Difieren de los márgenes continentales activos en que éstos últimos se asocian con la subducción. Los márgenes continentales pasivos a escala global son superficies que marcan la transición entre los océanos y los continentes sin que intervenga un límite de placa. Son la manifestación más amplia de la deformación extensional Mesozoica y Cenozoica en la litosfera continental. En la actualidad se encuentran bordeando las masas continentales que limitan el Atlántico, el Índico y el Ártico entre otros (Fig. 2.1).

Los márgenes pasivos conocidos como márgenes de rift o de tipo Atlántico, se desarrollan por adelgazamiento de la litosfera continental, seguido por una ruptura y la formación de nuevas cuencas oceánicas, bajo un régimen de esfuerzos tensionales, generalmente asociado con fallamiento normal, generación de magma y su emplazamiento, movimientos verticales de levantamientos o subsidencia, erosión y depositación de sedimentos [83, 96, 59, 108].

Entre los distintos márgenes pasivos de tipo Atlántico, existen grandes diferencias morfológicas, lo cual hace que no se los comprenda en su totalidad y quieran investigarse, sobre todo por su potencial como reservorios de petróleo.

Rifting continental y apertura involucran una interacción compleja de tectónica, magmatismo, geodinámica y procesos sedimentarios. Esta interacción resulta en una amplia gama de estilos de márgenes que van de estrechos a extendidos y de débilmente a fuertemente volcánicos. Los márgenes continentales pasivos se clasifican como volcánicos o no volcánicos de acuerdo a la cantidad de vulcanismo que haya ocurrido durante el rift. Globalmente los márgenes volcánicos representan entre el $75 \%$ y $90 \%$ de los márgenes 


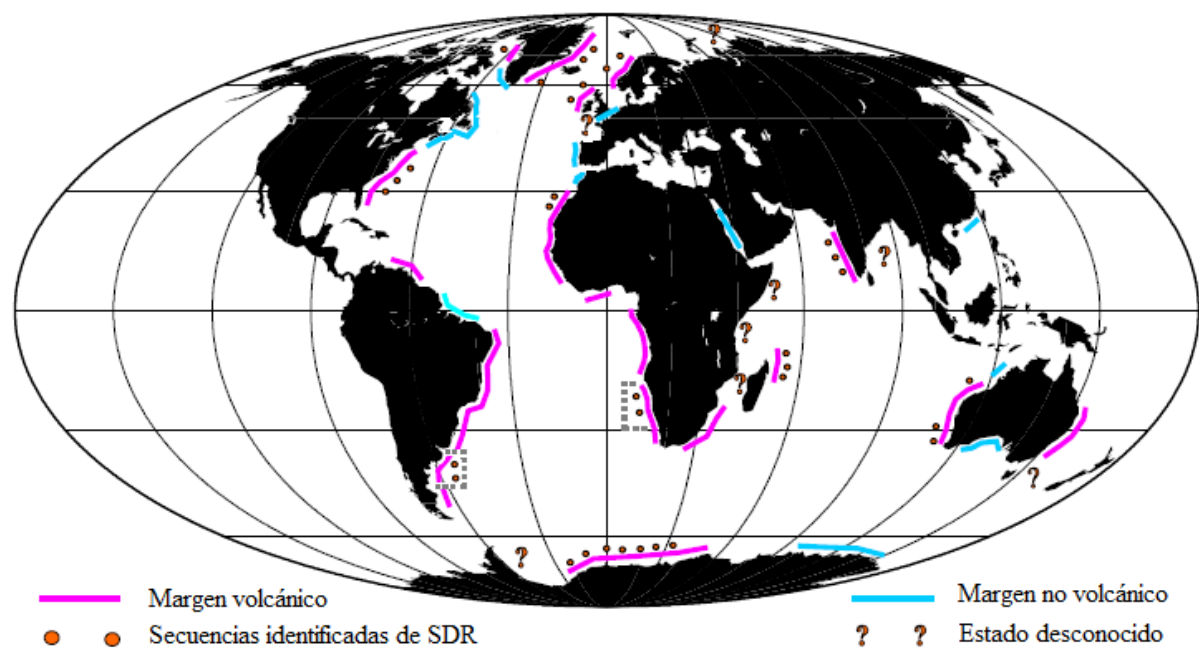

Figura 2.1. Distribución global de márgenes continentales volcánicos (rosa) y no volcánicos (azul). Modificado de Cuna [20]. Los márgenes volcánicos donde se han identificado secuencias de SDRs se han identificado con puntos naranjas. Las secciones encerradas por los rectángulos grises corresponden al margen estudiado en esta tesis (Argentino) y a su conjugado del lado Africano (Namibia).

continentales pasivos [31] (Fig. 2.1).

Los márgenes volcánicos están caracterizados por secuencias de reflectores buzantes (SDRs) formados por la erupción y subsecuente subsidencia de flujos volcánicos ocurridos durante el rifting y por gruesas capas de la corteza inferior con velocidades de onda $\mathrm{P}$ anormalmente altas $(>7.2 \mathrm{~km} / \mathrm{s})$ y altas densidades $\left(3000 \mathrm{~kg} / \mathrm{m}^{3}\right)$. Las capas de alta velocidad mapeadas debajo del talud continental varían de espesor entre 5-15 km., ambas capas de la corteza inferior y los complejos SDR están espacialmente asociados con la transición océano continente y se localizan entre la corteza adelgazada y la corteza normal [84]. La naturaleza y localización del límite entre la corteza continental y oceánica es de fundamental importancia para entender los procesos de rifting. SDRs y capas de la corteza inferior de alta velocidad en los márgenes volcánicos se han usado para sustentar la idea de plumas del manto involucradas en los procesos de rifting [84]. Los SDRs se distinguen por su forma de cuña y curvatura convexa de lavas buzantes hacia el mar, probablemente como resultado de la subsidencia diferencial de la fase inicial del rift.

Los márgenes del Atlántico Sur se formaron como resultado de la ruptura del supercontinente Gondwana. Finalmente, la apertura de Atlántico Sur tuvo lugar en el Cretácico inferior con lo que se infiere que la edad en que se separó Argentina/Uruguay de África/Namibia va desde 126 a 137 Ma [76]. La apertura del Atlántico Sur ocurrió diacrónicamente haciéndose más joven de sur a norte y puede describirse como una propagación hacia el norte de zonas de rift [31]. 


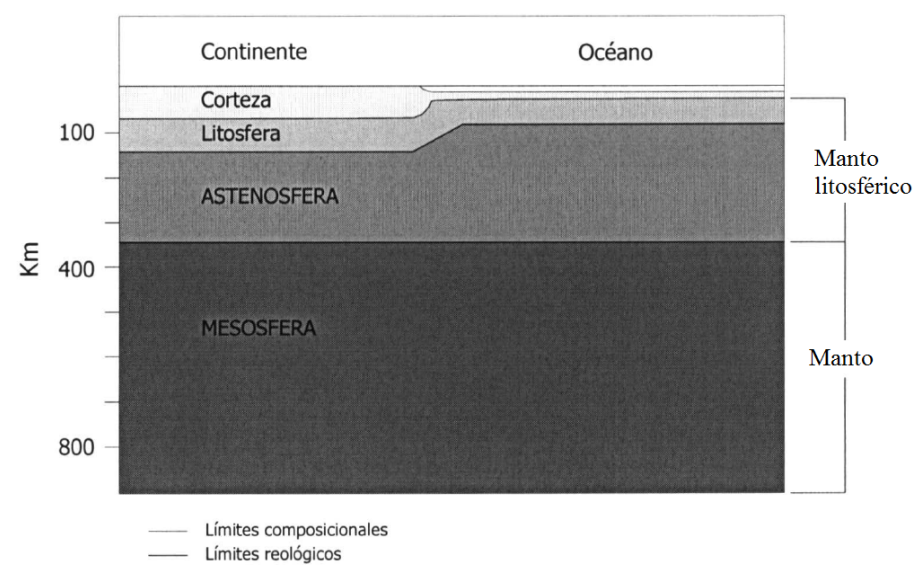

Figura 2.2. Principales límites composicionales y reológicos. El límite composicional más importante está entre la corteza y el manto. Modificado de Vargas [90, 1].

\subsection{Algunos conceptos sobre la litósfera y los me- canismos de subsidencia}

Las cuencas sedimentarias son regiones de la superficie de la Tierra que presentan una subsidencia que suele ser prolongada y que se origina por mecanismos que están relacionados con procesos del interior de la litósfera. La litosfera es relativamente rígida y esta compuesta por un número de placas que están en movimiento unas respecto de otras. Los procesos litosféricos son los responsables de la mayor parte de la actividad tectónica que se observa cerca de la superficie de la Tierra [90].

Se han establecido diferentes divisiones del interior de la Tierra en función de la composición química o del comportamiento mecánico de los materiales. Según la composición química se divide en corteza, manto y núcleo, mientras que en función de la reología o comportamiento mecánico de los materiales en litosfera, astenosfera y mesosfera (Fig. 2.2).

Según la composición el manto se compone básicamente de olivino y la corteza continental puede dividirse en dos: la corteza superior con propiedades físicas similares a la de los granitos, granodioritas o dioritas denominada "capa granítica", de unos 20-25 km de espesor que es cubierta por una delgada capa de rocas sedimentarias y la corteza inferior de composición basáltica pero que se va transformando en granulitas, ecoglitas o anfibolitas a medida que la presión y la temperatura aumentan por debajo de los $25 \mathrm{~km}$. Mientras que la corteza oceánica es una capa delgada de entre 4 a $20 \mathrm{~km}$ de espesor que contiene: una primera unidad de $0.5 \mathrm{~km}$ de espesor de sedimentos sin consolidar, una segunda unidad de composición basáltica con pillow lavas y material de las erupciones submarinas y una tercera unidad de gabros y peridotitas que puede ser la que genere los basaltos de la unidad anterior [90].

La reología de los materiales del interior de la Tierra depende de la temperatura como función de la profundidad, el tipo de roca y la cantidad de agua contenida en ellas. El principal límite reológico es el de la litosfera-astenosfera (Fig. 2.2). Una respuesta a la deformación de esta zona reológica son los mecanismos como la subsidencia o el 


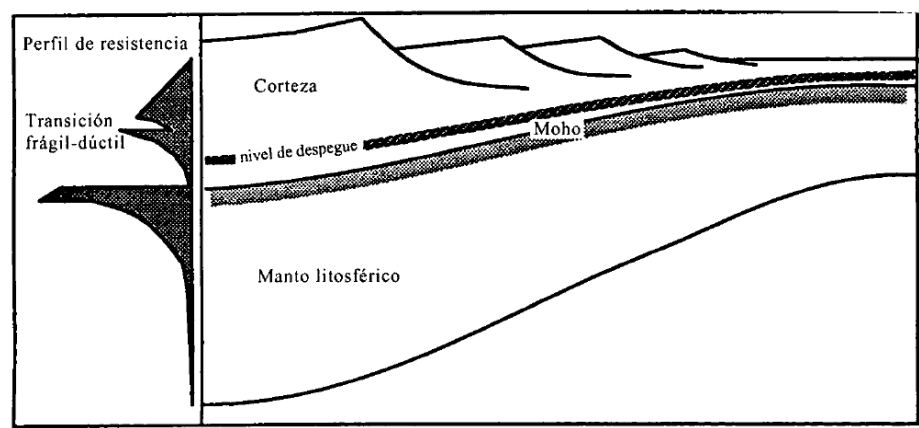

Figura 2.3. Perfil de resistencia de la litosfera y corteza oceánica. Extraído de Vargas [90].

a)
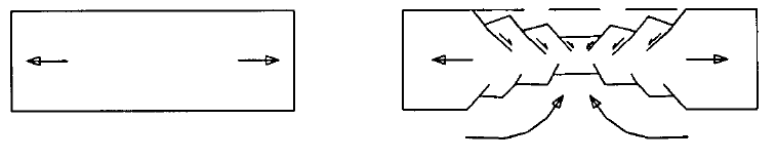

b)
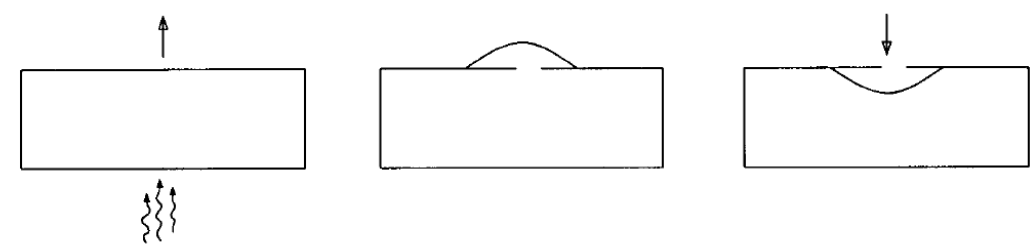

c)
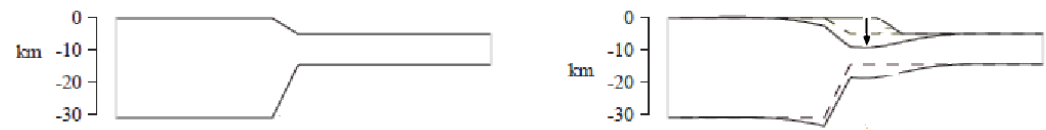

Figura 2.4. Mecanismo de extensión que genera subsidencia. (a) Extensión por tensión, (b) Subsidencia térmica. Extraído de Vargas [90] (c) Flexión por carga. Extraido de Watts [102].

levantamiento de las cuencas sedimentarias. La formación de cuencas sedimentarias y la acumulación de sedimentos en ellas son el resultado de la subsidencia, mecanismo relacionado a procesos que ocurren dentro de la litósfera como el estiramiento de la corteza y del manto litosferico.

La litosfera continental y la litosfera oceánica presentan diferente resistencia a la deformación con la profundidad, lo que se representa en los "perfiles de resistencia" (Fig. 2.3). La parte más resistente de la litosfera oceánica está en el manto entre los 20 y los 60 kilómetros de profundidad, a partir de la cual comienza a comportarse como dúctil [90]. La discontinuidad de Mohorovicic o Moho marca el límite entre la corteza y el manto, a travéz del cual hay un aumento de la velocidad de las ondas $\mathrm{P}$, lo que indica un aumento de la densidad de las rocas. Este nivel marca la transición del comportamiento frágil de la corteza inferior al comportamiento dúctil del manto. 


\subsection{Principales mecanismos de extensión}

Los principales mecanismos de extensión que generan cuencas sedimentarias de tipo distensivo y que explican la subsidencia en ellas son (Fig. 2.4) [90]:

- Extensión por tensión. La extensión de la corteza debida a procesos mecánicos de estiramiento genera una subsidencia controlada por fallas directas, mientras que en la litosfera se produce un levantamiento térmico (Fig. 2.4a). Las tensiones en el interior de la placa cortical provocan una serie de fracturas lineales que estiran y adelgazan la corteza, lo que genera el ascenso pasivo de la astenosfera $[59,7,8]$. Esta fase se ha denominado sin-rift.

- Subsidencia térmica. Se debe al progresivo enfriamiento de los materiales de la litosfera al situarse lentamente la astenosfera en el lugar que ocupaba originalmente (Fig. 2.4b), alcanzando el equilibrio térmico en unos 60 millones de años $[7,83]$. La"discordancia de ruptura" o breakup unconformity marca el momento en el que la subsidencia por tensión finaliza y comienza la subsidencia térmica. $\mathrm{Al}$ acumularse sedimentos en la cuenca generada mientras se va enfriando la litosfera, puede producirse un "efecto abrigo" o thermal blanketing [8] que provoca que la subsidencia sea menor y el enfriamiento sea más lento difundiensose lateralmente en lugar de verticalmente. Esta fase se ha denominado post-rift.

- Flexión por carga. El peso de los sedimentos acumulandos en la cuenca o el progresivo avance de una cadena montañosa en una zona compresiva deforma la corteza elásticamente dando lugar a un tipo de subsidencia por flexión (Fig. 2.4c). Esto es debido a que la corteza presenta una alta resistencia a las deformaciones horizontales, mientras que frente a las verticales se comporta elásticamente. La subsidencia que se genera se debe a reajustes isostáticos que pueden ser locales cuando existen fallas verticales que aíslan unos bloques de otros o flexurales a ambos lados de la cuenca, lo que genera cuencas más amplias que con el modelo de isostasia local [95]. Esta fase se puede dar tanto en las etapas de sin-rift como en las post-rift.

En general todos estos mecanismos actúan en el proceso de generación y evolución de una cuenca, aunque su importancia relativa varía con el tiempo. Dos modelos se han propuesto para explicar la extensión cortical y la formación de cuencas distensivas[90]:

- Cizalla pura (pure shear): en la litosfera se asume una deformación homogénea que implica una extensión instantánea y uniforme (Fig. 2.5a). La corteza superior se deforma de manera frágil mientras que la corteza inferior y el manto lo hacen de manera dúctil. En esta deformación no hay rotación de bloques y se genera una cuenca simétrica respecto a el eje central donde se produce la extensión.

- Cizalla simple (simple shear): se asume adelgazamiento de la litosfera por cizallamiento a lo largo de una falla maestra que corta parte o toda la litosfera, desarrollandose un rift asimétrico (Fig. 2.5b). La zona de cizalla transmite la extensión de la corteza superior a la corteza inferior y manto litosférico, siendo la extensión no uniforme y disminuyendo en la dirección de la cizalla. Las cuencas que se generan con este modelo tienen las siguientes características: 1) el flanco 
a) Cizalla pura

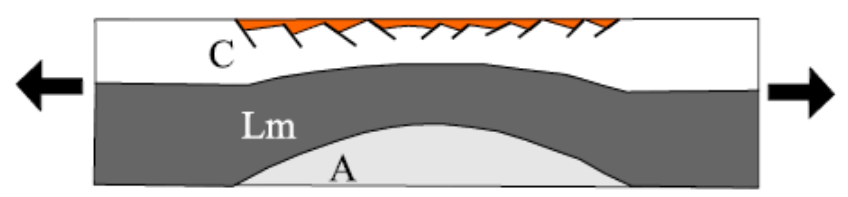

b) Cizalla simple

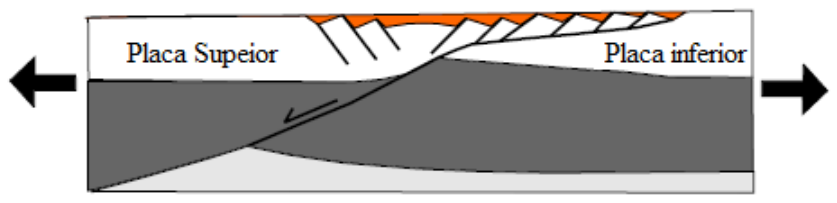

c) Cizalla pura y cizalla simple

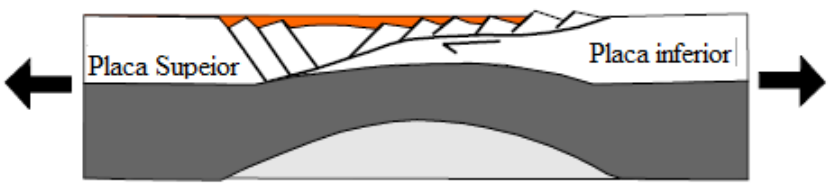

Figura 2.5. Diferentes modelos de extensión litosférica para la formación de cuencas de rift y márgenes pasivos: a) cizalla pura: con una capa superior frágil sobre una capa inferior dúctil. El estiramiento dúctil puede venir acompañado de dilatación debida a la intrusión de materiales fundidos, b) cizalla simple: con un despegue de bajo ángulo que divide a la litosfera en una placa superior (hanging wall plate) y una placa inferior (footwall plate). El adelgazamiento de la litosfera inferior se transmite a lo largo del plano de despegue, produciendo una sección fuertemente asimétrica de la litosfera [90] y c) combinación de un modelo de estiramiento de la corteza de cizalla simple y pura. Modificado de Cuna [20] quien se basó en [105, 55].

del lado hundido de la falla maestra asciende y la subsidencia térmica posterior, debida a la disminución de temperatura es más importante en la zona donde asciende el manto, 2) el máximo de subsidencia térmica no coincide con el máximo de subsidencia inicial o sin-rift y 3) hay rotación de bloques [105].

\subsection{Evolución de un margen continental pasivo vol- cánico}

El desarrollo de un margen pasivo volcánico (Fig. 2.6) es una consecuencia de la relativa magnitud del volcanismo durante la ruptura, la que depende de la temperatura y contenido de fluido de la astenosfera a lo largo del incipiente límite de placa y de la historia dinámica de la litosfera durante la fase de syn-rift [82]. Junto con los basaltos continentales, los plateau oceánicos y las cuencas de basalto oceánicos, los márgenes volcánicos constituyen la principal categoría de grandes provincias ígneas transitorias (LIPs, large igneous provinces, por sus siglas en inglés) [16], caracterizadas por voluminosos emplazamientos de rocas máficas en cortos intervalos de tiempo. Las diferentes 


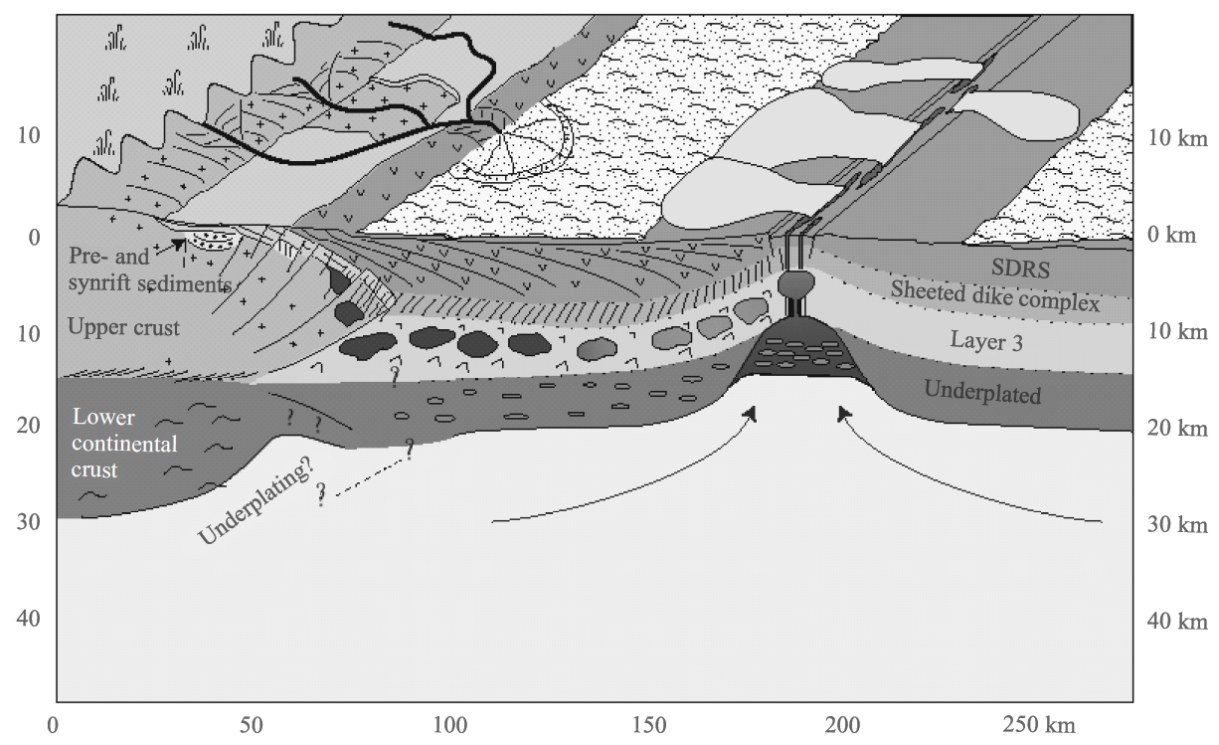

Figura 2.6. Diagrama de un margen volcánico idealizado. Modificado de Schmitd [82].

discordancias inmediatamente sobre las cuñas volcánicas SDRs marcarían el comienzo de la extensión del fondo oceánico en sentido estricto [44]. La litología de la secuencia de reflectores buzantes es aún desconocida. Los SDRs (Fig. 2.7) son representados como mezcla parcial convectiva causada por diferencias de temperatura lateral debida a los procesos de rift [64].

Hay otras propuestas de formación de los SDRs como la descompresión de mezcla y que el incremento del volcanismo se debería a plumas del manto [108], modelos de descompresión de mezcla combinados con la cabeza de una pluma resultan en un rápido y vigoroso volcanismo $[13,24]$, para el mecanismo de exceso de mezcla por descompresión se supone que una profunda y aguda ruptura de la litosfera forma una zona de rift propagada rápidamente.

La rápida propagación del rift en el margen Argentino estaría de acuerdo con la hipótesis de una pluma involucrada en la formación del margen volcánico [82].

Durante la apertura del océano Atlántico el volcanismo condujo a la formación de basaltos continentales como las provincias basalticas de Paraná en Sur América y Etendeka en Africa, ambas conectadas con la actividad del punto caliente de Tristan da Cunha y amplias zonas de secuencias de refectores sísmicos que inclinana hacia el mar. En la actualidad los SDRs se localizan a profundidades de entre 200 a $4500 \mathrm{~m}$. y se hallan en aproximadamente el $70 \%$ de los márgenes continentales del océano Atlántico [75]. Más adelante en este Capítulo, se presenta una visualización esquemática más moderna y completa publicada en [31], que fue hecha luego de un trabajo exhaustivo con una gran cantidad de datos sísmicos.

\subsection{Subsidencia en márgenes continentales pasivos}

Los márgenes continentales pasivos del tipo Atlántico se caracterizan por prismas de material volcánico cubiertos por sedimentos marinos que inclinan hacia el mar, sobre 
(1)

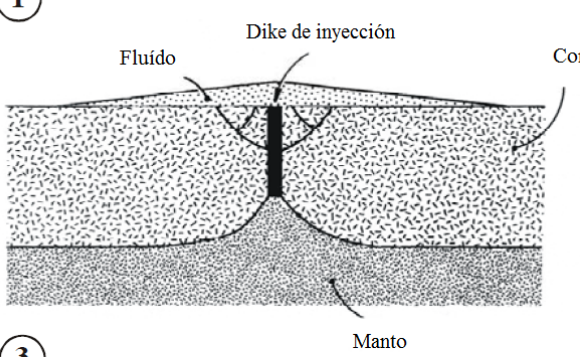

(3)

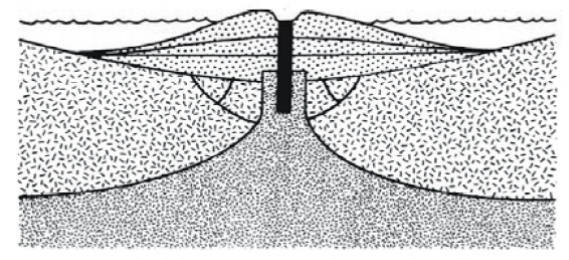

(2)

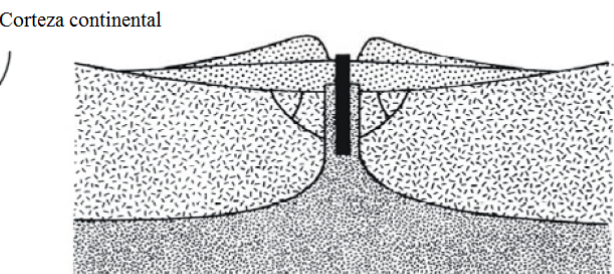

(4)

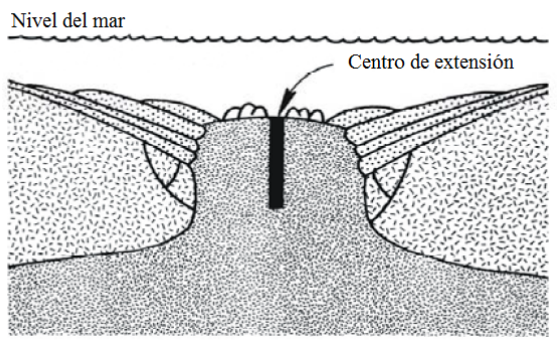

Figura 2.7. Modelo de emplazamiento de secuencias de reflectores buzantes que inclinan hacia el mar (SDRs). La evolución de los SDRs involucra domamiento e inyección de dique (1), sucesivos emplazamientos de flujo de lavas (1-4), los cuales luego del enfriamiento y carga con el flujo adicional sufren subsidencia (2-4) y se inclinan en la dirección hacia el mar. Modificado de Schmitd [82].

un basamento fallado, con secuencias sedimentarias syn-rift generalmente de origen continental. Es importante notar que los prismas de sedimentos post-rift corresponden predominantemente a depósitos de aguas someras [1] (Fig. 2.7).

Se han postulado varios mecanismos para caracterizar la subsidencia masiva de las fases syn-rift o post-rift desarrolladas en márgenes pasivos [1]. Estos mecanismos son:

Subsidencia debido a la carga sedimentaria: la sola carga de sedimentos puede producir hundimientos de dos o tres veces la profundidad del agua inicial. La carga sedimentaria aumenta la subsidencia y actúa aisladamente por lo que no es adecuada para explicar la formación de gruesas secuencias de depósitos de sedimentos de aguas someras.

El problema con este tipo de subsidencia es que no es fácilmente identificable habiendo sedimentos de aguas someras. Sólo puede funcionar en sedimentos que fueron depositados en aguas profundas inicialmente, ya que si la profundidad inicial del agua es menos de $200 \mathrm{~m}$, el efecto de carga de los sedimentos es despreciable.

Subsidencia debido a cambios de fase (de gabro a eclogita) en las rocas de la corteza baja o manto litósferico: aunque se ha postulado, no se conoce cuál de estos procesos puede extenderse lo suficiente como para ser el responsable de la subsidencia en los márgenes pasivos.

Subsidencia debido a creep de la corteza inferior dúctil hacia la oceánica: éste tipo de subsidencia es causada por una carga topográfica desigual a lo largo del margen [10].

Subsidencia debido al enfriamiento seguido al adelgazamiento litosférico: el ascenso de la astenosfera acompañado posiblemente por la intrusión de diques ultrabásicos o diapiros es seguido por contracción termal. El soporte para el crecimiento de los prismas 
sedimentarios podría ser la flexura de la litosfera. Algunos investigadores utilizan los modelos clásicos de placa elástica.

Es posible que más de un mecanismo operen en la evolución de la formación de un margen. De todos modos un modelo básico de extensión litosférica seguido por un enfriamiento es el punto de partida para cualquier análisis de subsidencia en un margen pasivo.

La acumulación de sedimentos en el margen pasivo representa una carga en la litosfera. La litosfera debería responder por flexura con una longitud de onda larga para una longitud de onda de la carga suficientemente corta.

Debido a que el estado inicial del rift es acompañado por fallamiento activo y flujo de calor alto, se asume que el modelo de Airy es el más adecuado en este período. Sin embargo los sedimentos post-rift son generalmente profundos y extensos sugiriendo que la flexura tiene lugar en algún estado posterior del rifting.

Watts [100] sugirió que los patrones característicos de superposición estratigráfica al este del Atlántico y en otros márgenes sugieren un incremento de la rigidez de la litosfera con el tiempo, lo cual sería el resultado esperado de una litosfera elástica calentada durante el estado de rift y el subsecuente enfriamiento.

Las observaciones geológicas básicas de los márgenes pasivos pueden resumirse así:

- Se superponen a los sistemas de rift tempranos que son generalmente subparalelos a los márgenes oceánicos, o más raramente a altos ángulos de los márgenes oceánicos (caso de los rift abortados de junturas triples, como a través del Benue, Nigeria), o a lo largo de zonas de fallas transformantes (Grand Banks y Golfo de Guinea);

- una fase de sedimentación syn-rift temprana puede reorganizarse en una fase tardía del drift y las dos están separadas por una discordancia (la discordancia de "ruptura");

- algunos márgenes pasivos exhiben un relieve subaéreo considerable al final del rift (en las cercanías de la mayor discordancia);

- secciones de sísmica de reflexión multicanal muestran que algunos márgenes pasivos pueden sustentarse por sistemas de fallamiento lístrico, aunque no siempre es el caso. En contraste la fase del drift está típicamente dominada por deformación controlada por la gravedad (tectónica de sal, diapiros de barro, depresiones, fallas de crecimiento lístrico en sedimentos blandos) [1];

- la historia de la subsidencia de los márgenes pasivos puede analizarse usando información de pozos y la técnica de análisis geohistórico "backstripping". Si se remueve el efecto de la carga de los sedimentos de la subsidencia, puede aislarse la "subsidencia tectónica".

\subsection{Margen continental argentino}

La plataforma continental del sector argentino de América del Sur tiene ciertos atributos por los cuales se la considerada como un márgen pasivo de placa inferior (lower 
plate passive margin) [78]. Sus características tectónicas actuales han sido controladas por su historia estructural previa, la que reguló los procesos de ruptura, flujo térmico y actividad magmática. La estructura cortical de la porción sur de Sudamérica es el resultado de un collage de bloques continentales que resultaron de la amalgamación de diferentes terrenos durante las orogenias precámbricas y fanerozoicas.

\subsubsection{Clasificación del margen continental argentino}

Se han identificado tres tipos de márgenes continentales: un margen continental pasivo volcánico, un margen cizallado y un margen combinado.

\section{Margen continental pasivo volcánico}

El margen continental pasivo volcánico se extiende desde los $35^{\circ} \mathrm{S}$ en el Río de la Plata (Límite con Uruguay) hasta los aproximadamente $48^{\circ} \mathrm{S}$ en la escarpa de Malvinas (ver Fig. 2.8).

El margen continental pasivo volcánico argentino tiene las siguientes características $[43,64,78]$ :

- El basamento de corteza continental se encuentra estructurado mediante fallas lístricas extensionales de rumbo paralelo y perpendicular al margen.

- Presencia de sistemas de rift abortados o aulacógenos caracterizados por asociaciones de fracturas perpendiculares ligeramente oblicuas al margen.

- Grandes zonas de discontinuidad o de transferencia ("tranfer zone", según Franke et al. [31]) perpendiculares a oblicuas al margen que afectan el basamento continental.

- Presencia de una potente cuña volcánica expresada en reflectores sísmicos intrabasamentales que inclinan hacia el mar (SDRs) $[54,45]$ que representan una fase de volcanismo extrusivo y un magmatismo intrusivo generado durante la fragmentación continental y subsidencia tectónica de la fase inicial del rifting $[108,46]$.

- Intrusiones de material magmático en la parte inferior de la corteza (suplacado magmático) asociadas a zonas de alta velocidad de onda sísmica (7.2 a $7.6 \mathrm{~km} / \mathrm{s}$ ).

- Una plataforma muy extensa que varía entre $160 \mathrm{~km}$ de ancho al Norte hasta más de $550 \mathrm{~km}$ frente a la cuenca Golfo San Jorge.

\section{Margen cizallado}

El margen cizallado se desarrolla entre las longitudes $58^{\circ} \mathrm{W}$ y $\operatorname{los} 38^{\circ} \mathrm{W}$ y aproximadamente a la latitud $49^{\circ} \mathrm{S}$ (ver Fig. 2.8) y se caracteriza por su tectónica transcurrente. El margen se extiende a lo largo de la escarpa de Malvinas y está contenido por el sector que incluye la meseta de Malvinas y el banco Maurice Ewing. Al norte de dicho sector se encuentra una zona compleja de fractura de transferencia denominada zona 


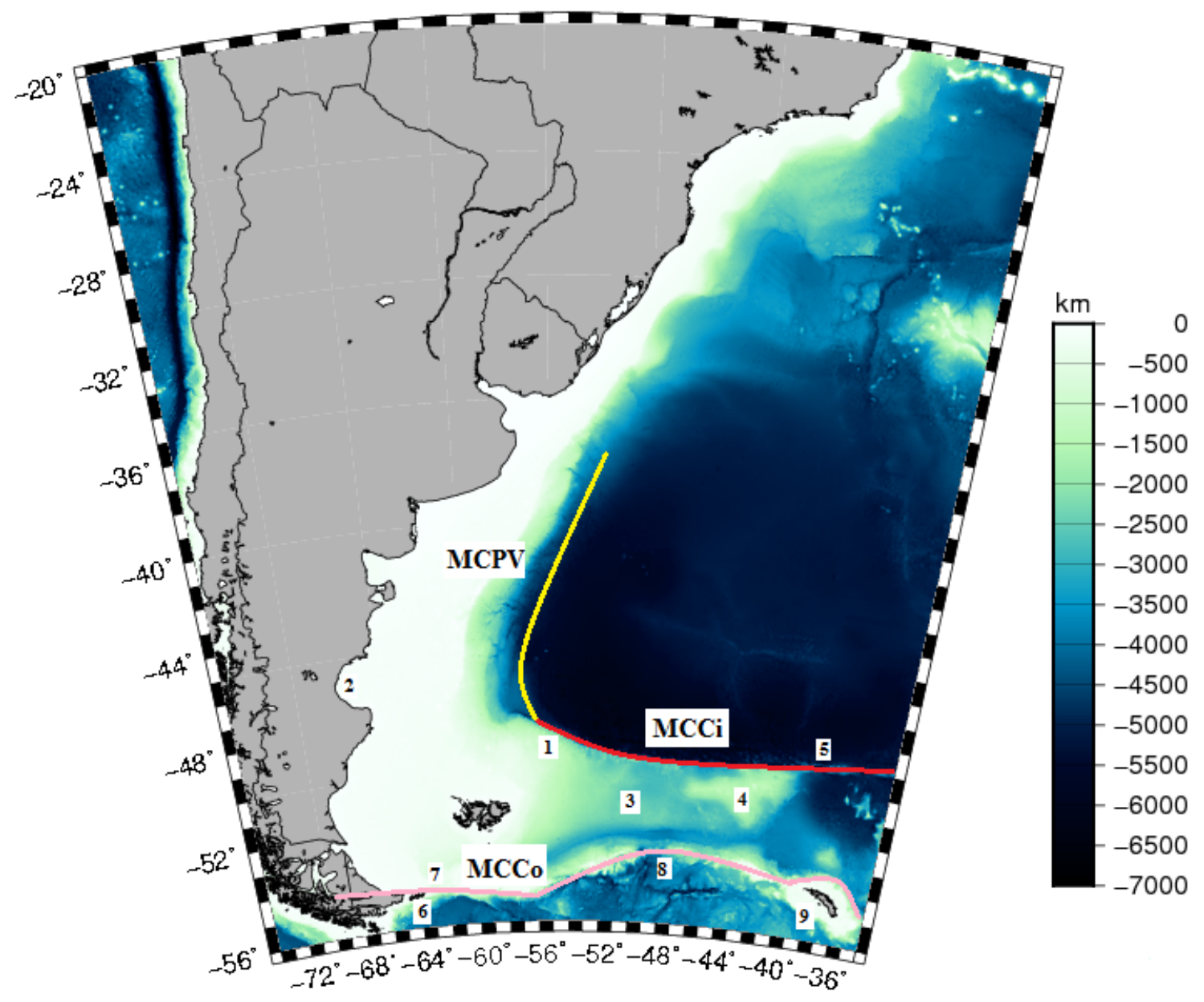

Figura 2.8. Mapa con los diferentes tipos de márgenes: MCPV: margen continental pasivo volcánico, MCCi: margen continental cizallado, MCCo: margen continental combinado, 1: escarpa de Malvinas, 2: Golfo de San Jorge, 3: meseta de Malvinas. 4: banco Maurice Ewing, 5: zona de transferencia de Malvinas, 6: isla de los Estados, 7: banco de Burdwood, 8: dorsal norte de Scotia, 9: islas Georgias del Sur. 
de transferencia de Malvinas, que se continúa hasta más al este del banco Maurice Ewing a través de la fractura de Malvinas. Su límite meridional está determinado por el surco de Malvinas, el cual representa el límite entre las placas de Sudamérica y de Scotia.

\section{Margen combinado}

El margen combinado (ver Figura 2.8) se localiza en la región de la isla grande de Tierra del Fuego, la isla de los Estados, el banco de Burdwood y la dorsal norte de Scotia, hasta la isla Georgia del Sur. Se extiende como una prolongación de la cordillera de los Andes al este del cabo de Hornos.

Este margen forma parte del sector septentrional de una estructura tectónica de mayores dimensiones, denominada el arco de Scotia [21].

\subsubsection{Generalidades tectónicas del margen continental argen- tino y su conjugado el margen continental de Namibia}

Los márgenes continentales pasivos conjugados tienen una asimetría evidente que permite dividirlos en dos tipos básicos: los de placa inferior (lower plate pasive margin) y los de placa superior (upper plate pasive margin)[78]. Es llamativa la diferencia entre márgenes continentales pasivos algunos limitados por sistemas montañosos, de otros caracterizados por extensas planicies costeras y amplias cuencas de rift bien desarrolladas. Ambos se diferencian por su flujo térmico y la heterogénea distribución del estiramiento, expresado por su factor $\beta$, cuando la cizalla que lleva a la fractura es controlada por cizalla simple (Fig. 2.5b) y no está homogéneamente distribuida como en la cizalla pura ((Fig. 2.5a). Esta cizalla simple se concentra a lo largo de zonas de debilidad previas, como zonas de sutura u otras discontinuidades corticales fundamentales. De acuerdo al modelo de cizalla simple propuesto por Wernike [105] discontinuidades corticales oblicuas conducen al desarrollo de sistemas asimétricos de rift caracterizados por hemigrabenes. El margen sudamericano al sur de la dorsal de Rio Grande-Walvis, se caracteriza por ser un margen de placa inferior que se habría comportado como una zona de transferencia, en contraposición al margen conjugado de Namibia y sector occidental de Sudáfrica, que se habría comportado como un margen de placa superior. Ramos [78] describe una serie de rasgos que permiten caracterizar el margen continental argentino como un margen de placa inferior, los cuales son:

- Desarrollo de una extensa plataforma: La fisiografía de la plataforma continental permite observar su desarrollo paulatino de norte a sur, a partir de la dorsal de Rio Grande y hasta la escarpa de Malvinas-Agullas. En este segmento el ancho varía de $160 \mathrm{~km}$ en la plataforma de Punta del Este, hasta más de $550 \mathrm{~km}$ frente a la cuenca del golfo de San Jorge. En contraste el margen conjugado frente a Namibia tiene una plataforma de un par de decenas de kilómetros.

- Planicies costeras en la terraza continental: El relieve de la planicie costera se caracteriza por ser una extensa llanura, que fue repetidamente inundada por las transgresiones cenozoicas como consecuencia de las variaciones del nivel del mar. 
La pampa deprimida frente a la bahía de Samborombón fue repetidas veces inundada por transgresiones durante tiempos históricos. Este suave relieve contrasta con el que se observa en Sudáfrica cuya divisoria de aguas se encuentra cercana a la costa Atlántica, con excepción del Rio Orange, que ha logrado atravesar el relieve costero. En gran parte de Namibia y Sudáfrica existe un importante relieve montañoso paralelo a la costa.

- Sistemas de hemigraben bien desarrollados: La plataforma argentina se caracteriza por el desarrollo de un sistema de rift, con hemigrabens orientados con polaridades alternantes que está pobremente desarrollado en el margen conjugado africano.

La orientación de estos sistemas de rift está fuertemente influenciada por la estructura previa, y varía de transversal a paralela al margen. En el margen de placa inferior la cizalla simple genera extensión en la parte superior de la corteza, que de acuerdo al modelo de Wernicke presenta el máximo factor de estiramiento $\beta$. Esto contrasta con el máximo atenuamiento que se observa en el manto litosférico, donde se concentra el estiramiento en los márgenes de placa superior.

- Sistema de dorsales periféricas externas: Los márgenes de placa inferior presentan como característica el desarrollo de altos o dorsos periféricos en la parte externa de la plataforma. Esta es una herencia del endomamiento previo y de la erosión producida en la discordancia de post-rift.

- Ausencia de magmatismo básico bien desarrollado: Se destaca el escaso desarrollo que tiene el magmatismo basáltico al norte del río Colorado y su ausencia al sur del mismo. En contraste el sector de Sierra Geral se caracteriza por mesetas basálticas donde extensos derrames de basaltos tholeíticos caracterizan el margen al norte de la dorsal de río Grande, pasando de sur a norte de un margen de placa inferior a uno de placa superior. Esta actividad magmática puede estar combinada con el empldesplazamiento de una pluma basáltica, coincidente con la región del máximo atenuamiento del manto litosférico.

En el margen conjugado los basaltos de Etendeska en Namibia, así como los basaltos del Karoo se ubican en el margen de placa superior conjugado.

- Desarrollo de extensos plateaus riolíticos: En la Patagonia extra andina hay una importante extensión areal de plateaus riolíticos.

El análisis de las estructuras del sector costero demuestra que hubo dos periodos de fallamiento extensional asociado a ésta actividad magmática, el primero y más importante fue en el Jurásico.

El desarrollo de este extenso plateau riolítico ha sido explicado como controlado por cizalla simple en un margen de placa inferior, donde el estiramiento se concentró en la corteza superior generando fusión parcial. En este modelo la fuente de calor es cortical y podría ser explicada por subplacado basáltico controlado por una cizalla maestra. Los plateau riolíticos de Somuncurá y el del Deseado se habrían producido en un corto intervalo de tiempo, lo que indicaría la existencia de una pluma astenosférica, aunque no hay registro de asociaciones petrológicas coherentes asociadas a la cabeza de una pluma o la cola de la misma. 
El engrosamiento cortical de la fase orogénica San Rafael, fue seguido de un colapso extensional que generó la provincia riolítica de Choiyoi. Esta extensión se propagó discontinuamente hacia el sur, en conjunto con el desmembramiento del supercontinente Gondwana, que se inició por la separación entre Gondwana occidental y oriental a los 170 Ma. Este desmembramiento estuvo controlado por una serie de discontinuidades corticales previas.

La sutura entre el cratón del Rio de la Plata y el terreno costero de Pelotas, que ivolucró una subducción a fines del proterozoico hacia el este actual [77, 29], coincide con la cizalla simple que reguló el inicio de la separación de Gondwana a esas latitudes. Hacia el sur del rio Colorado no se conocen las características del basamento que permitan inferir alguna relación similar.

\subsubsection{Características tectónicas del margen continental argen- tino}

La plataforma continental argentina puede ser dividida en tres sectores: los sectores norte y central corresponden a un margen pasivo de placa inferior, mientras que el sur esta regulado por un complejo fallamiento transforme [78].

- Sector norte

Como rasgo tectónico principal se destaca el predominio de estructuras paralelas a las zonas de sutura proterozoicas. El umbral de Martin García y las estructuras de las cuencas del Salado, Punta del Este y del Colorado, tienen un rumbo paralelo a la deformación penetrativa.Estas cuencas han sido interpretadas como aulacogénicas por su naturaleza ortogonal al margen.

Estudios gravimétricos realizados en la cuenca del Salado [48] muestran una fuerte anomalía de Bouguer residual en el eje de la cuenca, que ha sido interpretada como evidencia de atenuamiento cortical y emplazamiento de materiales más máficos en la base de la corteza, características en una cuenca aulacogénica.

Es interesante que la cuenca aulacogénica del Colorado presenta un rasgo gravimétrico de naturaleza mas asimétrico que la del Salado. La anomalía de Bouguer residual, una vez corregidos los efectos de los sedimentos, sugiere la existencia de un substrato con marcados contrastes de densidad al norte y sur del río Colorado. Una interpretación gravimétrica de la cuenca del Colorado señala como rasgo principal el contraste en el espesor cortical entre los márgenes norte y sur de la cuenca. Esta diferencia de espesor se puede deber tanto a la yuxtaposición de cortezas originalmente distintas o a un mayor atenuamiento cortical en el bloque superior de la zona de sutura. En los sistemas extensionales controlados por cizalla simple el atenuamiento cortical se concentra en la pared colgante de la zona de cizalla, donde se implantan los sistemas de rift.

- Sector central

El rasgo principal es el desarrollo submeridiano del sistema de rift subparalelo al margen de la plataforma.

Estas características se observan en tierra firme donde los sistemas de hemigraben están bien desarrollados en la región de Sierra Grande, así como en la planicie 
costera, los que han sido detectados a través de estudios gravimétricos y magnetométricos. Se ha notado un paralelismo entre el rumbo submeridiano de las estructuras de tierra firme al sur del río Colorado con las del margen externo de la plataforma continental.

Costa afuera este sistema se puede seguir a través de la cuenca de península de Valdés y la cuenca de Rawson.

Estudios sísmicos en la cuenca de San Julián han mostrado la existencia de un complejo de hemigraben en la continuación costa afuera del macizo del Deseado.

\section{- Sector sur}

Se caracteriza por el desarrollo de una escarpa de falla prominente correspondiente a la zona de falla Malvinas-Agulhas.

La plataforma de las islas y el plateau de las Malvinas adyacente hacia el este registran un sistema de rift de orientación general submeriadiana, que habrá estado activo durante el Jurásico medio a tardío.

Se observa hacia el sur la interacción con la región orogénica, que estuvo sometida a esfuerzos compresivos a partir del cierre de la cuenca marginal en la región cordillerana de Tierra del Fuego en el Cretácico superior.

\subsubsection{Anomalías magnéticas, gravimétricas y rasgos estructu- rales en el margen continental argentino}

En la Figura 2.9 se muestran los mapas de anomalías magnéticas: (Fig. 2.9a) obtenidas de una compilación de nuevos conjuntos de datos magnéticos [36] y el de anomalías gravimétricas de aire libre (Fig. 2.9b) obtenidas a partir de datos derivados de altimetría satelitaria [79] usando la versión 8.2 de Sandwell y Smith [79]. Sobre ambos mapas se ha superpuesto rasgos estructurales, por ej. sobre el mapa de gravedad se superpuso estructuras digitalizadas de las Figuras 8 y 10 de Ramos (1996) [78]. Dos sectores norte y sur, con respuestas magnéticas muy diferentes [35, 58, 37, 38] quedan separados por la denominada discontinuidad del Colorado (DC) dibujada con una línea segmentada gruesa que corta el margen al sur del Alto de Rawson.

Se ha trazado la ubicación de la anomalía G de acuerdo a la propuesta por Rabinowitz y LaBrecque (1979) [76], quienes la determinaron con los datos magnéticos y gravimétricos de cruceros disponibles. La anomalía G sigue el gradiente máximo de la anomalía isostática y tiene una expresión magnética asociada: una anomalía positiva elongada [36]. Según dichos autores la anomalía G marcaría la transición entre corteza continental y oceánica.

Una franja que marca la presencia de una cuña volcánica que según Hinz et al. (1999)[45] se implanta en el límite entre las cortezas continental y oceánica, está delimitada por dos curvas casi paralelas dibujadas en ocre (a) y en marrón (b). Esta cuña puede observarse en registros sísmicos como reflectores que inclinan hacia el mar, que se ubican en lo que técnicamente sería el basamento sísmico, y en su mayor parte coincide con la expresión magnética de la anomalía G.

La anomalía G no aparece en forma continua, sino que tiene algunas interrupciones. Al sur de los $45^{\circ}$, tanto la anomalía G original de Rabinowitz y LaBrecque (1979)[76] como la alineación magnética asociada [37] tienen un corrimiento hacia el este. La cuña 
volcánica tiene un corrimiento menor y una disminución de tamaño. La zona de cambio se encuentra en la discontinuidad del Colorado. En el océano están señaladas la serie M de anomalías mesozoicas de expansión del fondo oceánico, según la determinación de Ghidella et al. [36] basada en el nuevo mapa e inspirada en versiones anteriores [36] y de otros autores. Al sur de CD la anomalía más antigua es la M4; al norte aparecen hasta M10, en el borde de la cuña volcánica. M4 sigue siendo detectada en casi toda la zona. Las otras M, en cambio, tienen una extensión menor y se cortan al encontrar la cuña volcánica a la cual no son paralelas. Es de notar que tanto la cuña volcánica como las alineaciones (serie M) antiguas se interrumpen en la zona de DC y que en el mapa hay una anomalía negativa que es el efecto de borde que aparece cuando se llega a los bordes de los cuerpos magnéticos. Sobre esto puede verse más en Ghidella et al. [38].

Las anomalías gravimétricas de aire libre (Fig. 2.9b) tienen características muy interesantes. All este de la cuenca de Rawson y al sur de la discontinuidad del Colorado (Fig. 2.10) hay un mínimo relativo en el máximo gravimétrico que normalmente bordea el talud. Al norte de los $45^{\circ} \mathrm{S}$ y a una profundidad de entre 3500 y $4000 \mathrm{~m}$ hay un máximo relativo de gravedad de forma circular que está ubicado encima de una cuenca sedimentaria [66], intersectado por el final de la línea quebrada que marca la DC. Hacia el sur, en el borde de la cuenca Argentina, hay otro máximo relativo que forma una banda o festón, paralelo a la anomalía G. Las anomalías gravimétricas positivas sobre cuencas sedimentarias suelen ser indicativas de falta de compensación isostática, como ocurre en la cuenca del Salado [48], que se encontraría en subsidencia [48].

Como ya se señaló en la sección anterior se destaca como rasgo tectónico interesante en la zona norte la orientación de las cuencas casi perpendicular al margen y esta característica cambia al sur de DC donde las cuencas de Valdez y de Rawson son representantes de un sistema de rift subparalelo al borde de plataforma [78].

\subsubsection{Un modelo de apertura del océano Atlántico}

Durante el fenómeno astenosférico térmico que generó el emplazamiento de las provincias ígneas de Paraná-Etendeka, en el Jurásico tardío (aproximadamente a los 135 Ma), se generaron rupturas en la corteza; algunas de las cuales no alcanzaron la etapa de expansión oceánica y evolucionaron como cuencas aulacogénicas. La apertura del océano Atlántico Sur se desarrolló a lo largo de una dorsal de dirección aproximadamente norte - sur y se produjo con efusiones volcánicas detectables en registros sísmicos como reflectores intrabasamentales que inclinan hacia el mar (las cuñas volcánicas en las Figuras 2.11a y 2.11b). En la Figura 2.11a para orientación se han dibujado las cuñas volcánicas que están a ambos lados del océano. También se han trazado las anomalías G de ambos lados. Las cuencas Colorado (CC), Valdez (CV) y Rawson (CR) tienen aproximadamente su configuración actual (ver Fig. 2.10). El polo de rotación es $\left[45.5^{\circ} \mathrm{S},-32.20^{\circ} \mathrm{W}, 56.5\right]$ [36]. DC: Discontinuidad del Colorado. CO: cuenca de Outeniqua. También se observan: la cuenca de Claromecó y los sistemas Tandilla y Ventania, en África, los plegamientos de El Cabo y la faja conductora Cabo Sur en la cuenca de Karoo. En la Figura 2.11b al sur de la DC no hay anomalías anteriores a M4 del lado sudamericano (M4 está en rojo y las anteriores en marrón). En líneas negras con relleno verde aparecen los contornos de los valores máximos de gravedad. La dirección de 
(a)

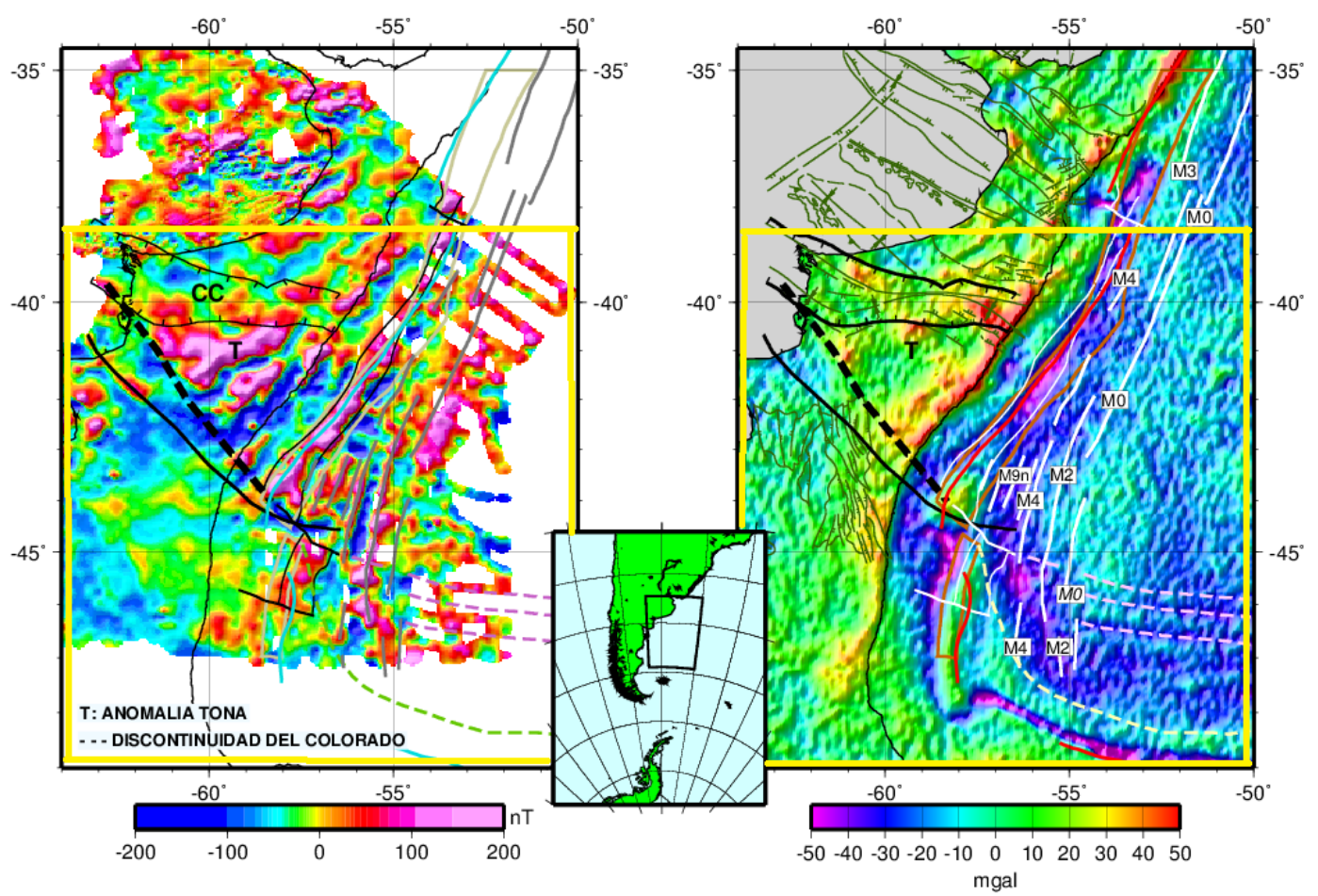

(b)

$35^{\circ}$ $5^{\circ}$

Figura 2.9. Anomalías magnéticas, gravimétricas y rasgos estructurales en el margen continental argentino

a) Anomalías magnéticas en relieve sombreado. b) Anomalías gravimétricas de aire libre derivadas de altimetría satelitaria [79]. El cuadro amarillo representa la región de estudio. Casi todas las líneas trazadas sobre las imágenes se encuentran en los dos mapas, a veces con diferente color para su contraste sobre el fondo. La anomalía magnética Tona y la discontinuidad del Colorado (DC) son de Ghidella et al. (1995) [35] y de Max et al. (1999) [58]. La línea negra cerca del borde de la plataforma es la isobata de $500 \mathrm{~m}$. En a) hay líneas negras que delimitan anomalías magnéticas positivas en el talud. Las mismas líneas están en blanco en b), el mapa de gravedad. Las cuñas volcánicas [45] están en ocre y en marrón. Notar que al norte de la DC la cuña y la anomalía positiva coinciden, pero no al sur. En azul en a) y rojo en b) se ha trazado la anomalía G de Rabinowitz y LaBrecque (1979) [76]. Las alineaciones mesozoicas están en gris en a) y en blanco en b), con algunas denominaciones. En el sector cercano a la cuña las anomalías van desde M10 a M4. Las líneas de rayas en color rosa son zonas de fractura interpretadas del mapa de gravedad. La línea de rayas que es verde en a) y blanca en b) es la interpretación de Ghidella et al. [36] del borde de la zona más influida por el arrastre de la transcurrencia en la zona de fractura de Malvinas (ZFM). En b) en color verde se han dibujado las estructuras digitalizadas de Ramos (1996) [78]. Modificado de Ghidella et al. [36]. 


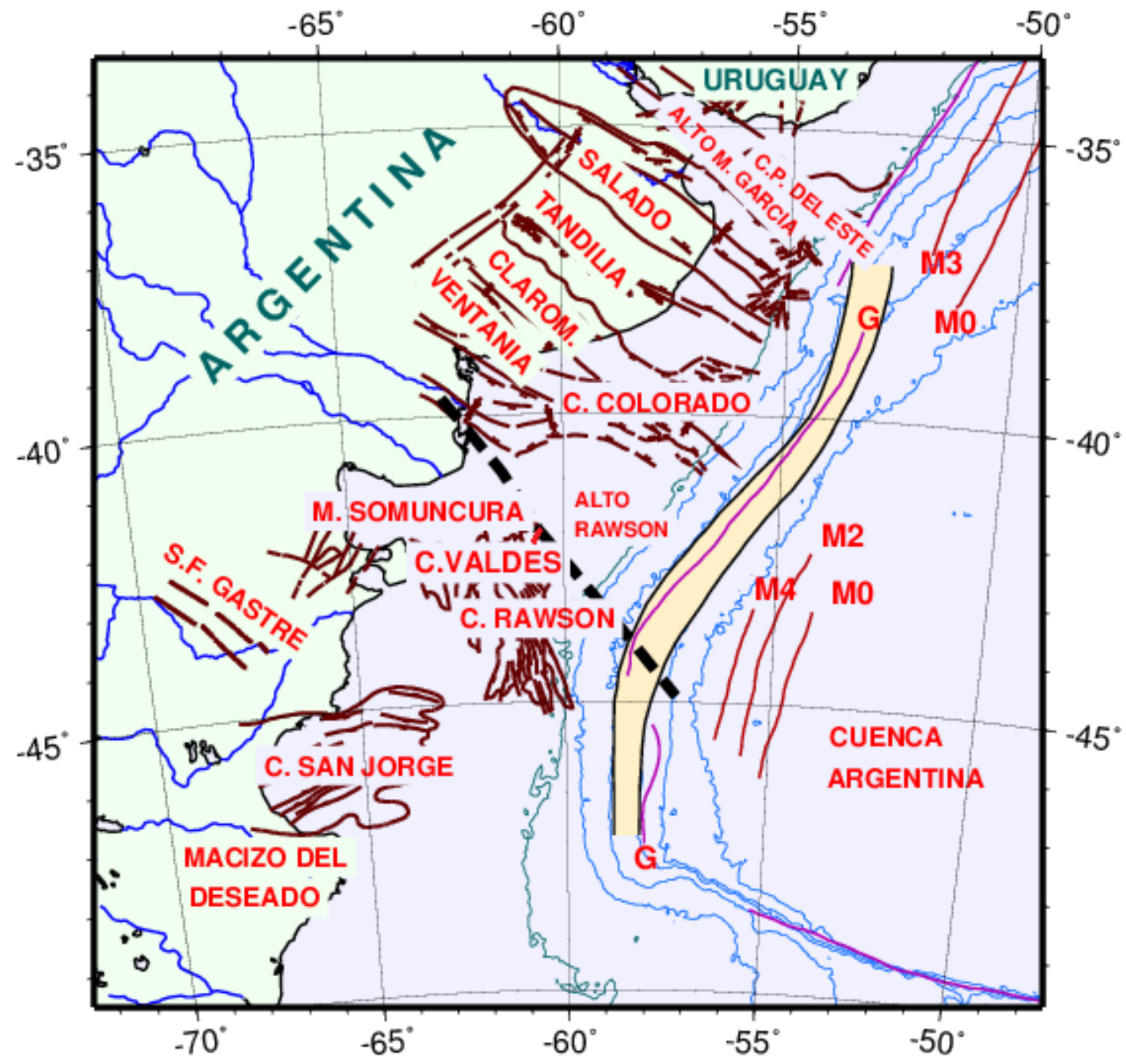

Figura 2.10. Ubicación de estructuras; las estructuras geológicas se digitalizaron de mapas en Ramos (1996) [78]; anomalías mesozoicas (serie M) según Cande et al. (1989)[14]; banda amarilla: cuña volcánica [45]. Línea segmentada negra: discontinuidad del Colorado. Modificado de la página web de Ghidella: http://www.martagh.com.ar/mararg/pictr2002/. 
apertura es la del estiramiento en las cuencas (doble flecha en (a)). El polo de rotación es $\left[49.3^{\circ} \mathrm{S}, 33.5^{\circ} \mathrm{W}, 54.3^{\circ}\right][36]$.

La ruptura inicial del margen argentino habría ocurrido a los $130 \mathrm{Ma}$, tal como lo sugieren las alineaciones magnéticas, en la zona ubicada actualmente en los $44^{\circ}-45^{\circ} \mathrm{S}$, comenzando a propagarse hacia el norte, y hacia el sur el espacio correspondiente fue aproximadamente compensado por extensión cortical en las cuencas Valdés, Rawson, y la cuenca Argentina. La Figura 2.11 muestra Sudamérica rotada hacia África para dos épocas: la de la ruptura inicial (a), en la cual las cuñas volcánicas se superponen o son una única cuña, y (b) la época de la alineación M4, 126.8 Ma, que sería la época a partir de la cual la apertura fue más regular. Ghidela et al.[36] han tratado de hacer ver los rasgos más destacados de la zona de discontinuidad. Los polos de rotación son los de Rabinowitz \& LaBrecque (1979) [76] algo modificados, pero no se ha segmentado Sudamérica, usando un solo polo para cada época. En el lado conjugado africano está la cuenca de Outeniqua, cuya dirección de extensión es la misma que la de la Fig. 2.10a y la cuña volcánica es más ancha y no se interrumpe como en el lado argentino, pero Ghidela et al.[36] no contaban con información actualizada. También aparecen anomalías mas antiguas hasta M11, a diferencia de lo que ocurre en el lado argentino. Consecuentemente la interpretación de Ghidella et al. (2006) [36] (Fig. 2.11) del lado africano tiene que ser revisada. Pero la zona al sur de DC podría haber sido influenciada por la vecindad del margen transcurrente en la zona de fractura de Malvinas, y es posible que se haya resistido a la ruptura por el mismo fenómeno que hizo que la plataforma de Malvinas no se rompiera.

\subsubsection{Segmentación del margen continental argentino}

Mediante el análisis de $25000 \mathrm{~km}$ de datos de sísmica multicanal, adquiridos por el BGR (Bundenstalt fur Geowissenshaften and Rohstoffe (Federal Institute for Geosciences and Natural Resources)) en el margen argentino, Franke et al. (2007) [31] interpretan que la apertura inicial del Atlántico estuvo acompañada de volcanismo en gran escala manifestado por los SDRs, y que el emplazamiento de esas cuñas volcánicas fue episódico. A lo largo del margen los autores encuentran variaciones en la arquitectura y volumen de las mismas, lo que ellos relacionan con una segmentación del margen en al menos cuatro secciones que van desde la zona de fractura de Malvinas hacia el norte, con "zonas de transferencia" que las separan, y que denominaron Colorado, Ventana y Salado (ver Fig. 2.12). Las zonas de transferencia describen lineamientos de corteza o discontinuidades en la arquitectura del margen y ellos proponen que estas zonas de transferencia perturban la propagación de rift y podrían representar la formación inicial de fallas oceánicas transformantes.

En general observan que el ancho de las cuñas de los SDRs disminuye hacia el norte dentro de los segmentos individuales. La diferente arquitectura volcánica de los segmentos y los diferentes volúmenes de magma extruido puede indicar que el emplazamiento del material volcánico fue controlado por el marco tectónico y la configuración de la litósfera pre-rift en los segmentos individuales del margen. Franke et al. [31] sugieren a la descompresión adiabática y la generación de mezcla de fuentes someras como lo que puede explicar las distintas variaciones a lo largo del margen. 
(a)

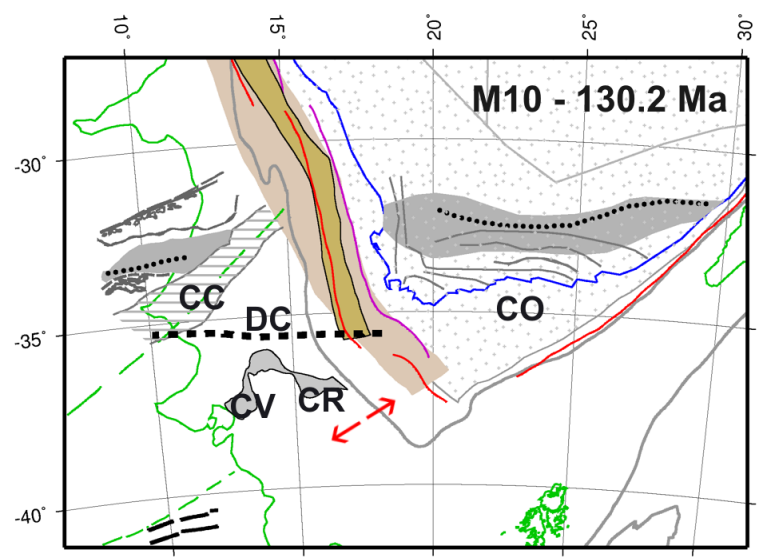

(b)

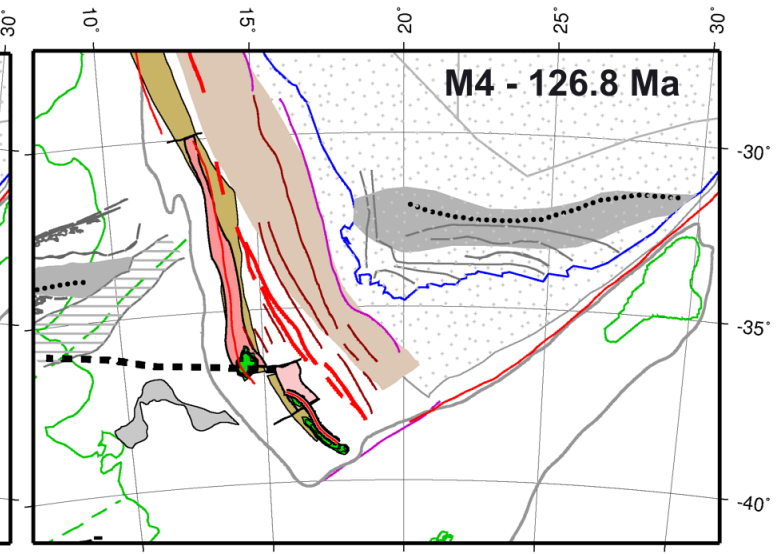

Figura 2.11. (a) Sudamérica rotada hacia África para la época de inicio de la apertura [36]. Para orientación se han dibujado las cuñas volcánicas que se encuentran a ambos lados del océano. También se han trazado las anomalías G de ambos lados. Las cuencas Colorado (CC), Valdés (CV) y Rawson $(\mathrm{CR})$ tienen aproximadamente su configuración actual. DC: discontinuidad del Colorado. CO: cuenca de Outeniqua. También se ve la cuenca de Claromecó y los sistemas de Tandilia y Ventania. En África los plegamientos del El Cabo y la faja conductora Cabo Sur en la cuenca del Karoo. (b) Sudamérica rotada hacia áfrica para la época de la anomalía M4. Al sur de la DC no hay anomalías anteriores a M4 del lado sudamericano (M4 está en rojo y las anteriores en marrón). En líneas negras con relleno verde aparecen los contornos de los valores máximos de gravedad. La dirección de apertura es la del estiramiento de las cuencas (doble flecha en (a)). Modificado de Ghidella et al. [36]. 


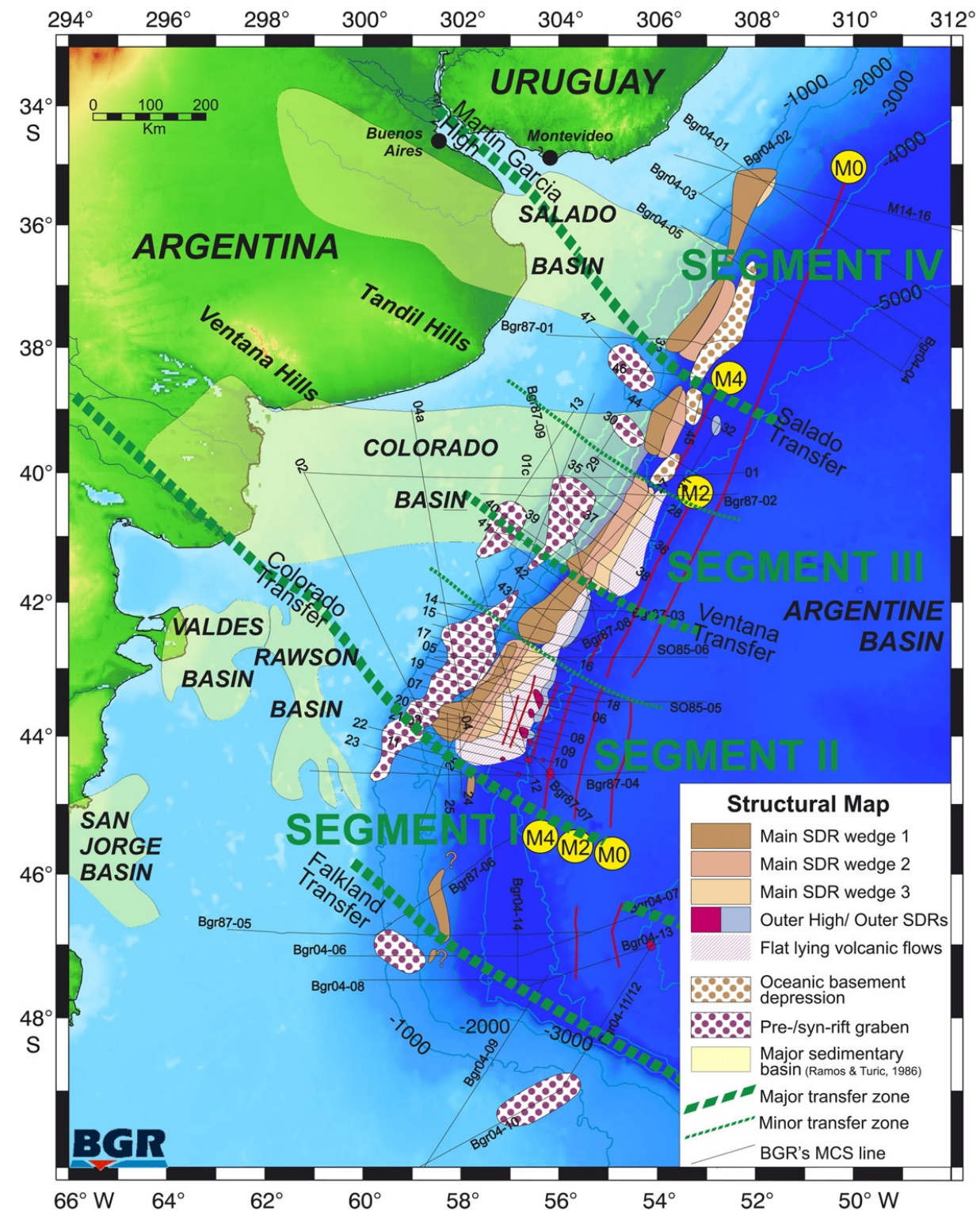

Figura 2.12. Mapa estructural mostrando la distribución de manifestaciones volcánicas de gruesas cuñas de secuencias de reflectores que inclinan hacia el mar (SDRs), superficies volcánicas/magmáticas adicionales, depresiones del basamento oceánico y grabens pre-/syn-rift. Las zonas de transferencia y la segmentación del margen se interpretan a partir de variaciones en las características tectónico-volcánicas, distribución de sedimentos post-rift, se indican datos de campos potenciales y estudios previos [31]. 


\subsubsection{Arquitectura y estilo de las cuñas volcánicas del margen continental argentino}

Franke et al. [31] han identificado hasta cinco secuencias adyacentes de SDRs en perfiles sísmicos en el área del margen continental argentino, separadas por fuertes discordancias, mapeando con claridad un máximo de tres (ej. Fig. 2.14). Los SDRs individuales son convexos hacia arriba y muestran el patrón característico de reflectores divergentes [45, 63]. Cada sección se engrosa hacia abajo y aumenta en inclinación. Las secuencias individuales de los principales SDRs se han trazado en profundidad, hasta casi el nivel del Moho, cuando no están enmascaradas por múltiples. Las cuñas exteriores, (Fig. 2.14) en contraste son más pronunciadas y las reflexiones internas muestran, en general, una extensión de solo 0.5 s (tiempo de ida y vuelta, TWT). Franke et al. (2007) [31] infieren que estas cuñas han sido emplazadas bajo condiciones subacuáticas debido a subsidencia progresiva de la corteza volcánica-magmática. Los flujos individuales están separados por altos topográficos locales. En general el tope de las cuñas SDRs es plano.

En la inclinación hacia el mar de estos SDRs ocasionalmente se presentan altos volcánicos exteriores (volcanic outher highs) y en algunos casos cuñas de reflectores que inclinan hacia el mar adicionales, aunque poco desarrollados en lo concerniente a espesor y amplitud.

Los flujos de SDRs individuales tienen en promedio sólo $6 \mathrm{~m}$ de espesor, siendo muy delgados para poder resolverse como reflectores individuales para el sistema de adquisición empleado [31].

Fuertes reflectores arqueados, separan la secuencia a lo largo de la pendiente e indican variaciones en la continuidad del emplazamiento de las cuñas individuales. Hay varias razones que pueden explicar la formación de discontinuidades como son: los cambios en el volumen y rapidez de la producción del magmatismo, el medio ambiente volcánico (geometría de apertura, relación con el nivel del mar, etc.), también cualquier deformación simultánea con el volcanismo o posterior y la velocidad y cantidad de subsidencia. Aunque no se tiene un control de la subsidencia se puede asumirse que fue lo suficientemente continua en los segmentos individuales del margen. Se sugiere [31] que las diferentes cuñas limitadas por fuertes discontinuidades son la expresión de un emplazamiento episódico. De acuerdo a la interpretación de Franke et al. (2007) [31], la cuña de SDRs localizada más hacia el continente es la que fue emplazada primero. Después de que esa cuña fue emplazada, posiblemente erosionandose y subsidiendo, se emplaza la próxima cuña. Aunque este flujo cubre parcialmente la primera cuña, la parte más importante de la cuña está localizada hacia el mar respecto al flujo del primer SDRs. Se propone el mismo mecanismo para el emplazamiento de la siguiente cuña de SDRs. 


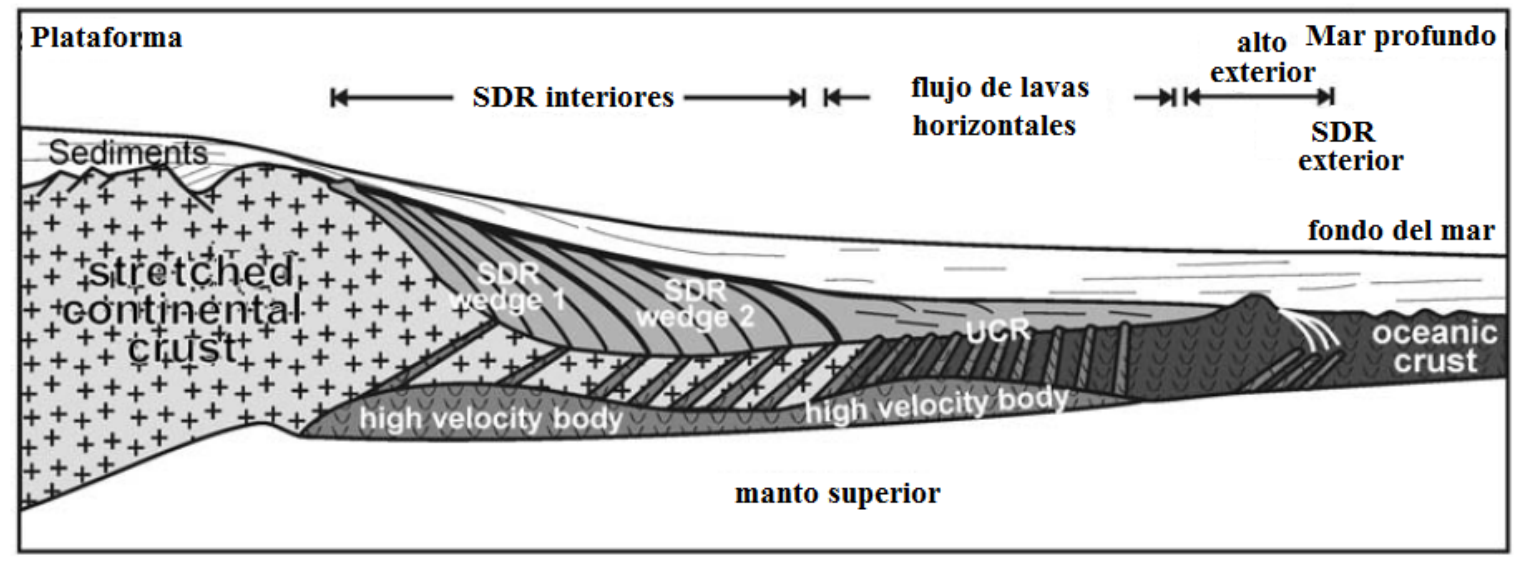

Figura 2.13. Esquema que ilustra las principales superficies volcánicasmagmáticas en el margen continental argentino (no está a escala). Por debajo del talud hay uno o múltiples reflectores que inclinan hacia el mar (SDR) y un cuerpo de la corteza inferior de alta velocidad. Prominentes superficies hacia el mar son otros cuerpos de alta velocidad y las Reflexiones de la Corteza Superior (UCR, por sus siglas en inglés). El área más lejana de los SDRs está definida por la ocurrencia de altos exteriores y cuñas de SDRs antes de que comience la corteza oceánica. Modificado de Franke et al. [32].

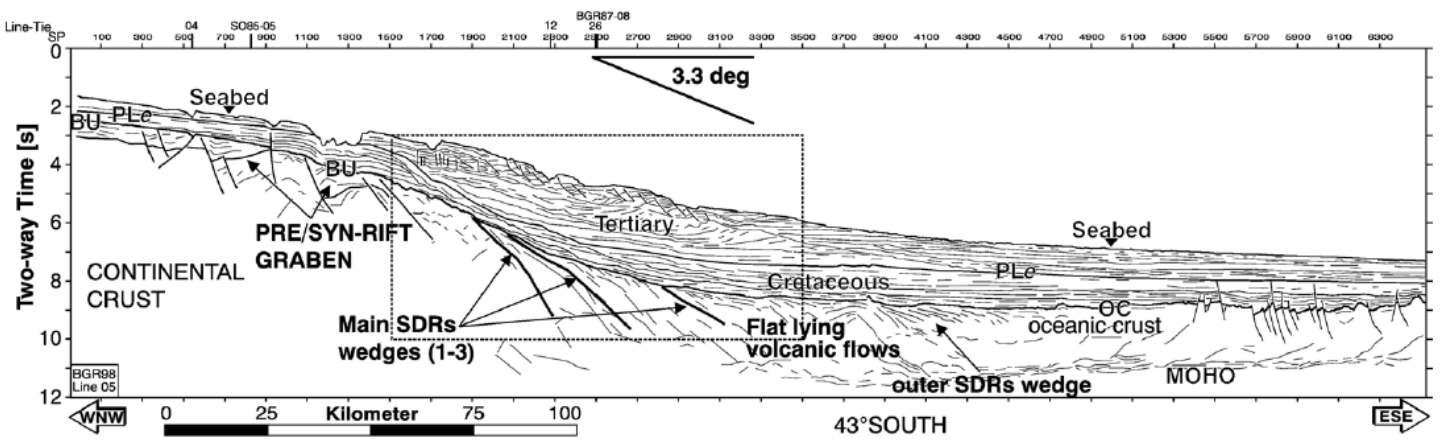

Figura 2.14. Perfil BGR98-05 situado en el segmento II del margen (Fig. 2.12). Múltiples SDRs están separadas por fuertes discontinuidades. OC: corteza oceánica, BU: discontinuidad de ruptura, PLe: equivalencia Pedro Luro, SDRs: reflectores intrabasamentales que inclinan hacia el mar. Modificado de [31]. 



\section{Capítulo 3}

\section{Base de datos de gravedad}

Comenzamos este Capítulo con la idea de llevar a cabo una compilación de datos gravimétricos de barco con el objetivo de disponer de una base organizada de datos de gravedad, no sólo para esta tesis, sino para trabajos posteriores. Llevamos a cabo esta tarea parcialmente dado que con los datos de barco de los que disponemos no se logra una cobertura total y homogénea de la zona. Realizamos la compilación en forma parcial y está lista para ser aumentada a medida que aparezcan nuevos datos. Simultáneamente notamos a través de la comparación con los datos de barco que las compilaciones derivadas de altimetría satelitaria poseían una resolución y precisión suficiente para el objetivo de nuestro trabajo por lo que en adelante decidimos utilizar dicha compilación para el desarrollo de la tesis.

\subsection{Bases de datos de gravedad consistente y docu- mentada}

En distintas décadas se han realizado alrededor del mundo mediciones de gravedad marina desde plataformas a bordo de barcos. Debido a errores del instrumental, de navegación y otras fuentes de error, existen diferencias significativas entre las mediciones de gravedad realizadas en distintos cruceros. La exactitud de los datos de barco está limitada principalmente por el error de navegación que afecta el cálculo del efecto Eötvös [23].

En contraste con las anomalías de gravedad medidas a bordo de barcos, las anomalías de gravedad derivadas de multimisiones satelitales tienen una cobertura homogénea y global, disponibles en grillas de dominio público, las que han sido calculadas, salvo algunas diferencias, usando la misma combinación de datos y distintas filosofías computacionales de cálculo [26]. Para resolver cuál de éstas grillas es superior en precisión o exactitud o es más representativa de los datos del margen continental argentino, planteamos la comparación con las anomalías de gravedad marina obtenidas desde gravímetros a abordo de barcos. Disponemos de datos del Bureau Gravimétrique Internacional (BGI) y de las campañas BGR87, BGR98 (1 y 2) realizadas por el Federal Institute for Geosciences and Natural Resources. En el presente Capítulo se comparan cuatro grillas recientes de anomalías de gravedad derivadas de altimetría satelital en el margen continental argentino y zona oceánica adyacente llamadas DNSC08GRA 
[2], DTU10GRA [5] del DTU Space (National Space Institute) y las versiones V16.1 y V18.1 de Sandwell [79, 80].

Como existen diferencias entre las distintas fuentes de datos, para decidir sobre la exactitud y precisión de las anomalías de gravedad de barco hace falta contar con que éstas últimas constituyen una fuente de datos de mayor precisión, para lo cual ésta base de datos debe ser consistente y documentada. En una base consistente los datos han sido cuidadosamente editados, se han eliminado datos ruidosos propios del sistema de registración influenciados por movimientos de rotación del barco y se les ha hecho algún tipo de corrección por cruces (cross-overs), primero para evaluar el acuerdo interno de los datos y segundo entre diferentes campañas para evaluar el acuerdo externo.

Inicialmente utilizamos los datos de altimetría satelital para descartar líneas de barco muy ruidosas o con algún error sistemático que produce diferencias muy grandes respecto a los datos derivados de altimetría. Para cada juego de comparación tenemos la estadística de las trazas individualizadas, lo que posibilita identificar aquellas que producen las mayores diferencias; visualizando gráficamente dichas trazas puede identificarse la mala calidad del dato ya sea por falta de continuidad en la registración o por la alta variabilidad del mismo (posible ruido).

Como primer paso en el proceso de compilación (luego de la comparacion inicial con la altimetría satelital), comenzamos con la edición manual línea por línea de los datos de barco de cada campaña, y luego hacemos la comparación nuevamente con los datos derivados de altimetría.

\subsection{Anomalías de gravedad derivadas de altimetría satelital}

Para calcular anomalías de gravedad a partir de altimetría desde satélite algunos autores usan técnicas que incluyen:

- 1) la conversión de la altura del geoide marino (corregido por la topografía de la superficie del mar) usando la fórmula inversa de Stokes en el dominio espectral vía FFT [2],

- 2) la conversión de la deflexión de la vertical vía integración de la ecuación de Laplace [79]. En estos métodos se usa la técnica remove-restore, que hace uso de un modelo geopotencial durante la conversión de la deflexión de la vertical a anomalías/perturbación basado en la transformada rápida de Fourier (FFT). En el uso de FFT existe un problema asociado con el efecto de borde dado que no se tienen datos altimétricos en tierra, y también resulta más problemático el grillado de datos. Además, distintos grupos tienden a usar algoritmos diferentes de grillado de datos como splines o mínimos cuadrados por colocación, por lo que se esperan diferencias entre grillas de anomalías de gravedad debido exclusivamente a la técnica usada [26]. 


\subsection{Datos utilizados}

\subsubsection{Grillas de anomalía de aire libre derivadas de altimetría satelital}

\section{DNSC08GRA}

Las anomalías de gravedad se derivan de la ondulación del geoide usando técnicas de FFT en el dominio de la frecuencia. Se incluye un filtro tipo Wiener para suavizar el campo de gravedad, que toma el valor 0.5 para la frecuencia de corte determinada empíricamente; longitudes de onda menores están dominadas por ruido y causan el llamado "efecto de piel de naranja" de los campos de gravedad derivados de altimetría [2]. El filtro fué diseñado usando 10 años de observaciones de ERS-2 en las que las regiones de alta variabilidad de la altura de la superficie del mar se filtraron más que las de regiones calmas. Luego de aplicar el filtro se restaura la contribución de las anomalías de gravedad del modelo EGM2008 [69]. La mejora en precisión y exactitud de este modelo en general siguen los mismos pasos que la versión V18.1 de Sandwell [81]. Se aplica una técnica nueva de análisis descendente y ascendente de traza (doubleretracking) a los datos de ERS-1, la que difiere de las aplicadas por Sandwell [79] dado que incluyen un paso adicional de tolerancia múltiple adaptativa a las condiciones locales. Este paso adicional cubre de un 6 a $9 \%$ más datos en regiones de aguas someras y regiones polares.

En altas latitudes se incluyen datos de altimetría de ICESAT (Ice, Cloud, and land Elevation Satellite) y datos marinos en el océano ártico del proyecto de gravedad del ártico $(\operatorname{Arc} G P)$ que dan exactitud y mayor cobertura al campo de gravedad. Usando éstos datos es posible alcanzar las regiones polares de $82^{\circ} \mathrm{N}$ donde no se tienen datos de altimetría previa.

Se usa el nuevo modelo geopotencial global EGM2008 seis veces más preciso espacialmente que el EGM96 [53]. Esto da más exactitud en el proceso remove-restore que con cualquier otra señal geopotencial. Se utiliza un modelo de topografía dinámica media DOT07A junto al EGM2008 para corregir la altura de la superficie del mar. Este modelo está disponible en http://www.space.dtu/ con resolución 1 minuto de arco.

DTU10GRA es el campo de gravedad global sucesor del DNSC08GRA. Tiene mejoras en la edición, retracking (analisis de la curva de la intensidad de la señal recibida sobre el tiempo con el fin de obtener parámetros adicionales o mas precisos acerca de la superficie del medio medida como por ej: hielo, ríos, océano [33]) en ERS-1 GM, contempla información del cambio climático (retiro de hielos) y aunque aún falta mejorar el rango y correcciones geofísicas (marea oceánica, ect.) e implementar una corrección anual con datos estacionales, representa una mejora respecto del DNSC08GRA ya que incluye retracking en ERS-2 y en ENVISAT [3].

\section{V16.1 y V18.1}

Son series recientes de anomalías de gravedad marina de Sandwell [80], de resolución 1 minuto de arco.

Se utilizaron perfiles de altimetría satelital de las misiones Geosat Geodetic y de Ers-1 que se convirtieron a grillas de gradiente vertical de gravedad y anomalía de gravedad. 
(a)

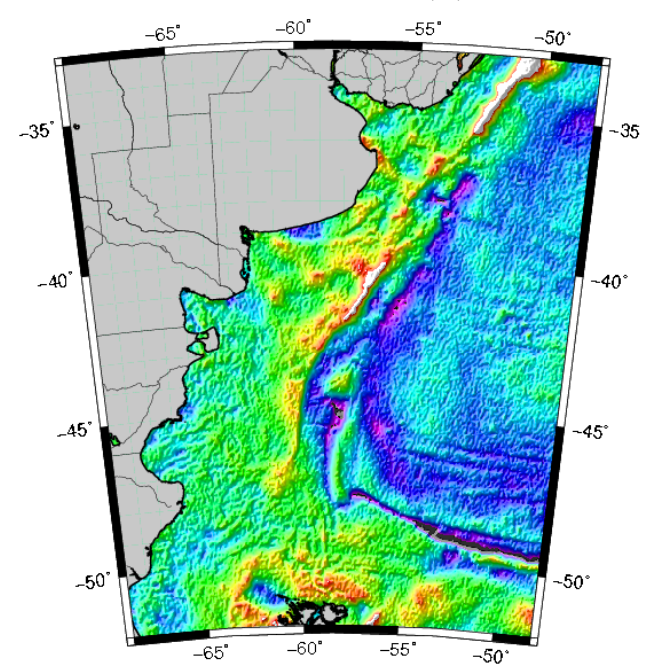

(b)

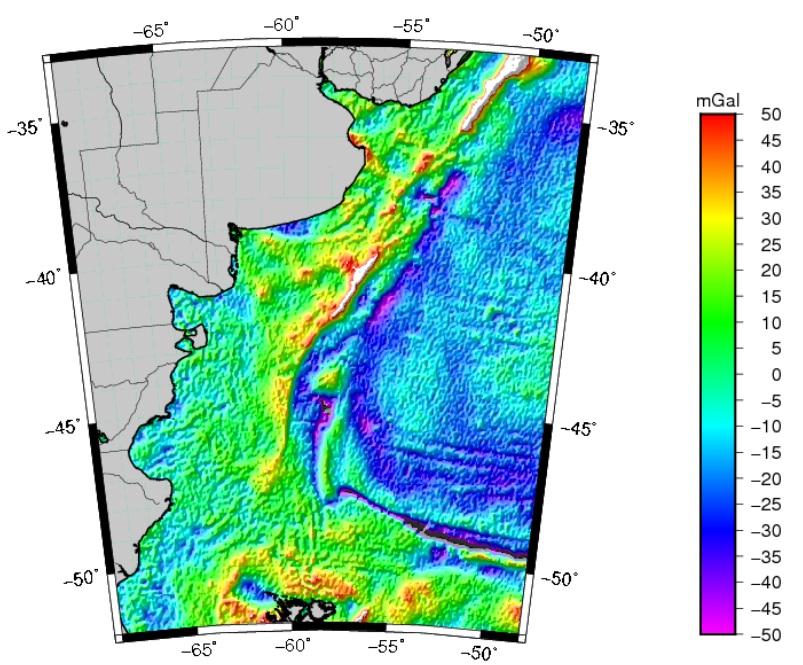

Figura 3.1. Anomalía de aire libre versión V16.1 (a), izquierda y V18.1 (b), derecha.

Las anomalías de gravedad se calcularon utilizando FFT inversa en la ecuación de Laplace con deflexión de la vertical, usando un modelo de referencia en la implementación de la FFT y el método remove-restore: el campo de referencia es removido de la deflexión de la vertical y es restaurado en la conversión a anomalías de gravedad [79]. Se han utilizado los modelos EGM96 para la versión V16.1 (Figura 3.1a) y EGM2008 para la V18.1 (Figura 3.1b).

En la versión V18.1 se aplicaron mejoras vinculadas al reproceso de datos de ERS-1 y Geosat/GM, empleando retraking en datos de 11 meses de ERS-1 y 18 meses Geosat/GM, dando un rango de precisión de un $40 \%$ y $27 \%$ respectivamente [80]. Se usó el nuevo modelo geopotencial EGM2008 en el método de remove-restore, obteniéndose así una resolución de anomalías de gravedad de 5 minutos sobre tierra (EGM2008) y 1 minuto sobre los océanos, otorgando entonces continuidad a la transición tierra-océano. Se usó interpolación bi-armónica en la construcción de grillas de deflexión de la vertical residual. Se incluye un modelo de la topografía dinámica media (MDOT) junto al EGM2008 para corregir una porción de las pendientes de la superficie del mar asociada a corrientes limítrofes occidentales resultando esto en una mejora de 6-10 mGal en la exactitud de las anomalías de gravedad en ésas áreas. Se puede disponer de estas grillas en ftp://topex.ucsd.edu/pub/global_grav_1min/.

\subsubsection{Datos de barco en el margen continental argentino}

Por acuerdos de cooperación existentes tenemos acceso a dos conjuntos de datos de dos campañas marinas llevadas a cabo por el BGR, Bundesanstalt für Geowissenshaften and Rohstoffe (Federal Institute for Geosciences and Natural Resources). Disponemos también de los informes técnicos de los cruceros, que fueron realizados en 1987 (BGR87) y en 1998 (BGR98), respectivamente. De dichos informes sabemos que para BGR87 se usó un gravímetro marino Bodenseewerk modelo KSS Nº serie 30. El crucero BGR98 
fué desarrollado en dos etapas, diciembre de 1998 (BGR98-1) y enero de 1999 (BGR982) con un gravímetro marino LaCoste \& Romberg modelo $\mathrm{S}$, del tipo aire-mar, $\mathrm{N}^{\circ} \mathrm{de}$ serie S-105. El sistema de referencia utilizado para los datos gravimétricos fué el I.G.N.S 71 (International Gravity Standarization Net 1971). Se les ha aplicado la corrección de Eötvös con los datos de navegación, corrección por drift instrumental y substracción de la gravedad normal WGS 67 (World Geodetic Sistem 1967). La navegación se apoyó en el sistema de posicionamiento GPS (Global Positioning System), y el sistema de satelite de navegación (Transit Satellite System o Navy Navigation Satellite System, NNSS).

Otro conjunto de datos utilizados es el BGI, que es una compilación de varios años de toma de medidas (1941-1988), las cuales fueron registradas por el Bureau Gravimetrique International en los que entre otros datos se incluyen el número de fuente, coordenadas (latitud y longitud), gravedad observada en microGal, anomalía de aire libre, anomalía de gravedad de Bouguer, estimación de los errores en las anomalías, y un código que representa el instrumento utilizado entre otros datos.

Se tiene un número de 7537 observaciones de BGI, 10465 de BGR87, 95566 de BGR981 y 76086 de BGR98-2 en un área comprendida entre las latitudes $36^{\circ} \mathrm{S}$ y $48^{\circ} \mathrm{S}$ y las longitudes $62^{\circ} \mathrm{W}$ y $48^{\circ} \mathrm{W}$.

Las anomalías de aire libre medidas por las campañas de barco están referidas a GRS67 (Sistema de Referencia Geodésico 1967). Para referirlas al GRS80 (Sistema de Referencia Geodésico 1980) utilizamos la siguiente fórmula:

$$
\Delta g_{G R S 80}=\Delta g_{G R S 67}+\gamma_{G R S 67}-\gamma_{G R S 80}
$$

donde $\Delta g_{G R S 80}$ y $\Delta g_{G R S 67}$ son las anomalías de gravedad en el sistema GRS80 y GRS67, respectivamente, y $\gamma_{G R S 80}$ y $\gamma_{G R S 67}$ los valores de gravedad normal en los sistemas GRS80 y GRS67, respectivamente.

$$
\begin{aligned}
& \gamma_{G R S 67}=978031,8\left[1+0,0053024 \operatorname{sen}^{2}(\phi)-0,0000059 \operatorname{sen}^{2}(2 \phi)\right][\mathrm{mGal}] \\
& \gamma_{G R S 80}=978032,7\left[1+0,0053024 \operatorname{sen}^{2}(\phi)-0,0000058 \operatorname{sen}^{2}(2 \phi)\right][\mathrm{mGal}]
\end{aligned}
$$

en las que $\phi$ es la latitud geodésica, 978031.8 es el valor de gravedad normal en el ecuador para el sistema GRS67 y 978032.7 para el sistema GRS80.

Estos datos no están aún editados ni corregidos por cruces por lo que no pueden proveer un control definitivo de la exactitud de las grillas de altimetría satelital en la región del margen continental argentino. La comparación directa de las diferentes fuentes de datos podrá mostrar niveles de acuerdo (quizás precisión) pero no exactitud.

En las Figuras 3.2 y 3.3 observamos la distribución de las trazas de cada campaña gravimétrica: en la Figura 3.2 las del BGR98 en su etapa 1 (BGR98-1), en la Figura 3.2b las de BGR98 en su etapa 2 (BGR98-2), en la Figura 3.3a las de BGR87 y en la Figura 3.3b la del BGI. 
(a)

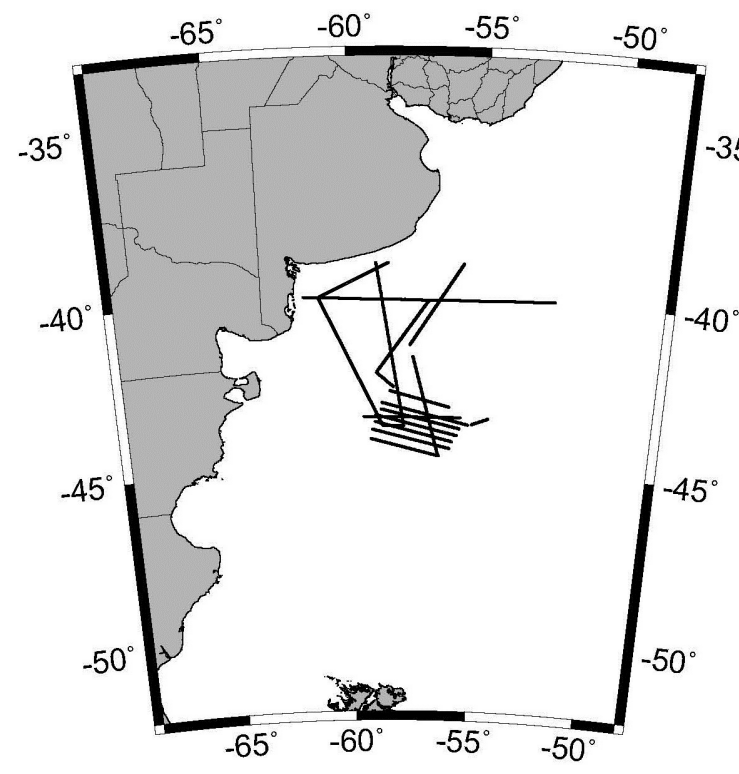

(b)

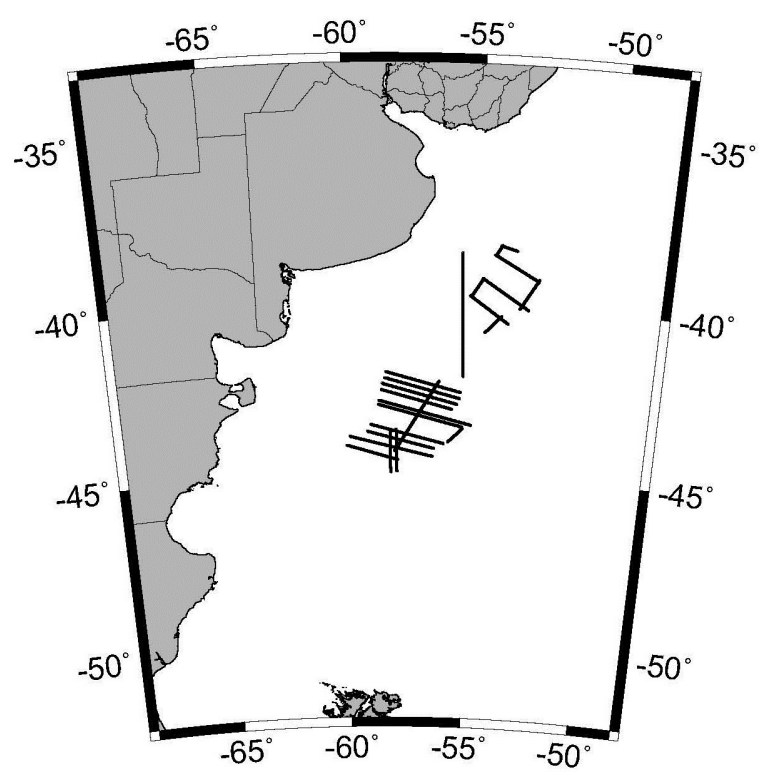

Figura 3.2. Derrotas de barco de BGR98-1 (a), izquierda y BGR98-2 (b), derecha.

(a)

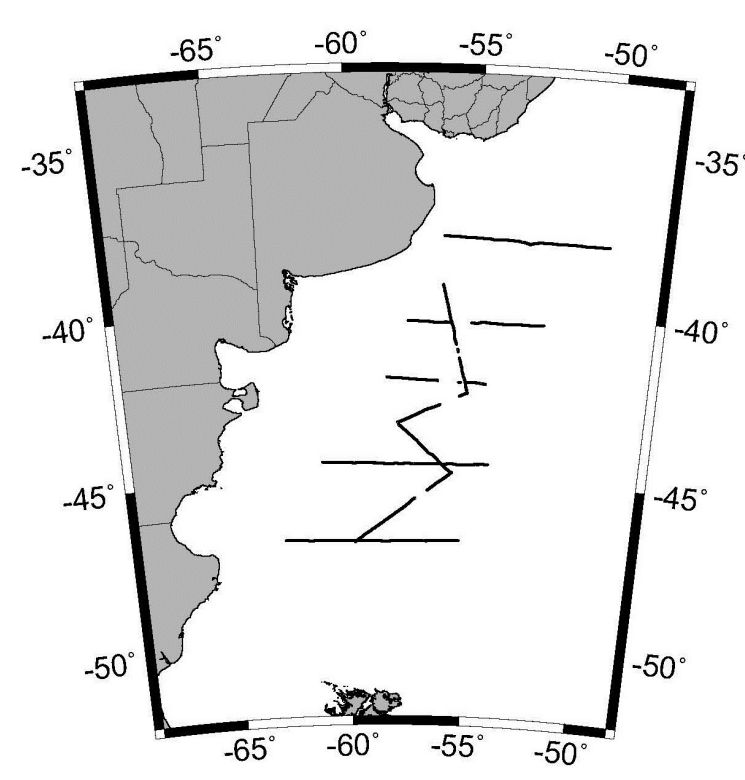

(b)

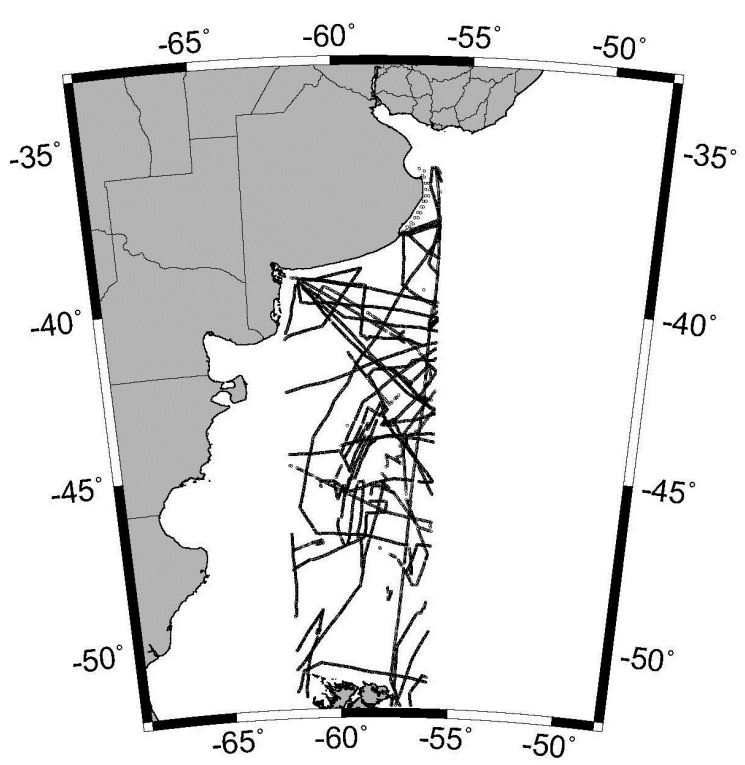

Figura 3.3. Derrotas de barco de BGR87 (a), izquierda y BGI (b), derecha. 


\begin{tabular}{c|l|l|l|c}
\hline Grillas & \multicolumn{1}{|l}{ máximo } & \multicolumn{1}{l}{ mínimo } & \multicolumn{1}{c}{ media } & \multicolumn{1}{c}{$\boldsymbol{\sigma}$} \\
\hline SyS16.1 & 84.60 & -105.80 & -3.07 & 19.83 \\
SyS18.1 & 95.13 & -103.83 & -3.32 & 19.45 \\
DNSC08 & 95.35 & -102.94 & -3.27 & 19.46 \\
DTU10 & 95.52 & -102.51 & -3.28 & 19.40 \\
\hline
\end{tabular}

Tabla 3.1. Estadística de las anomalías de gravedad derivadas de altimetría. Unidad: [mGal].

(a)

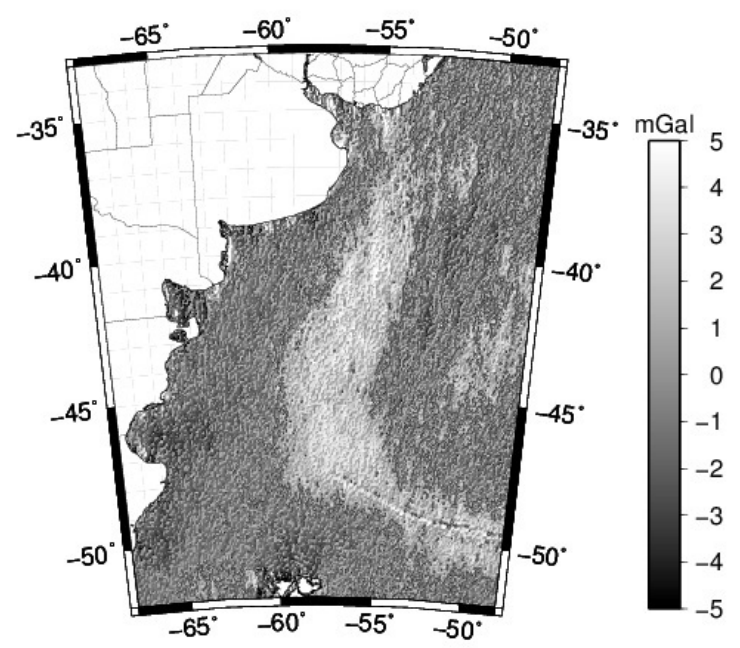

(b)

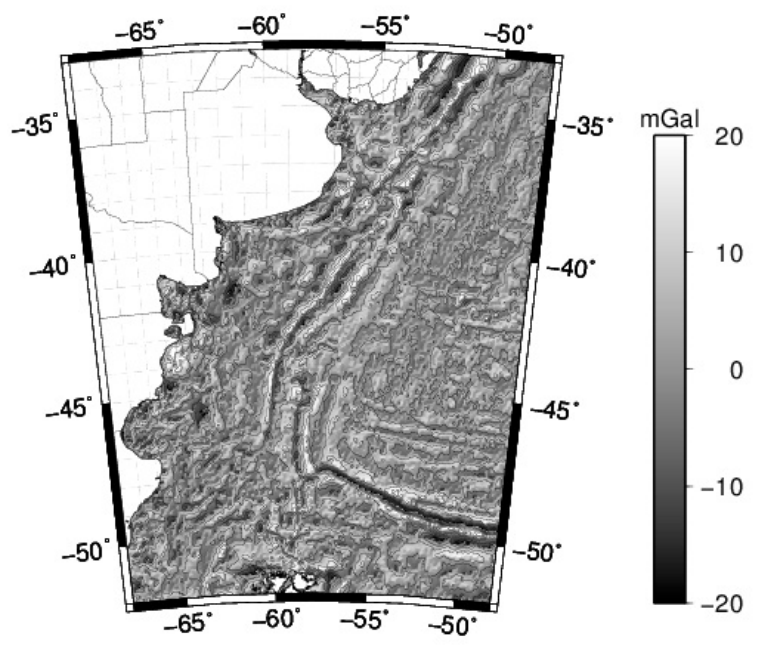

Figura 3.4. Diferencias entre grillas de anomalías de aire libre SyS18.1SyS16.1 (a), izquierda y EGM2008-EGM96 (b), derecha.

\subsection{Comparación}

\subsubsection{Comparación entre grillas de anomalías de gravedad de- rivadas de altimetría satelital}

Extrajimos grillas rectangulares de bases de datos altimétricos en el área de estudio, a las que se hace referencia en el texto con los nombres SyS16.1 (V16.1), SyS18.1 (V18.1), DNSC08 (DNSC08GRA) y DTU10 (DTU10GRA).

Como las anomalías de gravedad hacen uso del modelo EGM96 (V16.1) o EGM2008 (v18.1, DNSC08GRA y DTU10GRA) en tierra, en cada grilla removimos ésta contribución usando la rutina grdlandmask de GMT 4.5.3 [106]. Con éste programa se puede generar una grilla auxiliar en la que los nodos en tierra emergida (continentes e islas) son reemplazados por NAN (identificador de ausencia de datos) y los nodos en mar toman el valor 1. Esto constituye una máscara que utilizamos como un filtro espacial para eliminar los datos de tierra con la rutina grdmath, quitando así su influencia antes del cálculo de la estadística.

En la Tabla 3.1 mostramos la estadística de las grillas de anomalías de gravedad derivadas de altimetría satelital, en el área de los mapas de la Figura 3.5. Podemos observar que en la Tabla 3.1 hay un buen acuerdo de unas a otras lo que es de esperar dado el uso de los mismos datos. Tanto V16.1 como V18.1, DNSC08GRA y DTU10GRA 


\begin{tabular}{c|l|l|r|r}
\hline Grillas & máximo & \multicolumn{1}{c}{ mínimo } & \multicolumn{1}{c}{ media } & \multicolumn{1}{c}{$\boldsymbol{\sigma}$} \\
\hline SyS18.1-SyS16.1 & 46.45 & -31.66 & -0.240 & 2.19 \\
DNSC08-SyS16.1 & 46.67 & -34.82 & -0.200 & 2.95 \\
DNSC08-SyS18.1 & 28.28 & -24.05 & 0.040 & 1.87 \\
DNSC08-DTU10 & 14.05 & -19.83 & 0.003 & 1.00 \\
\hline
\end{tabular}

Tabla 3.2. Estadística de diferencias entre grillas de anomalías de gravedad derivadas de altimetría. Unidad: [mGal].

(a)

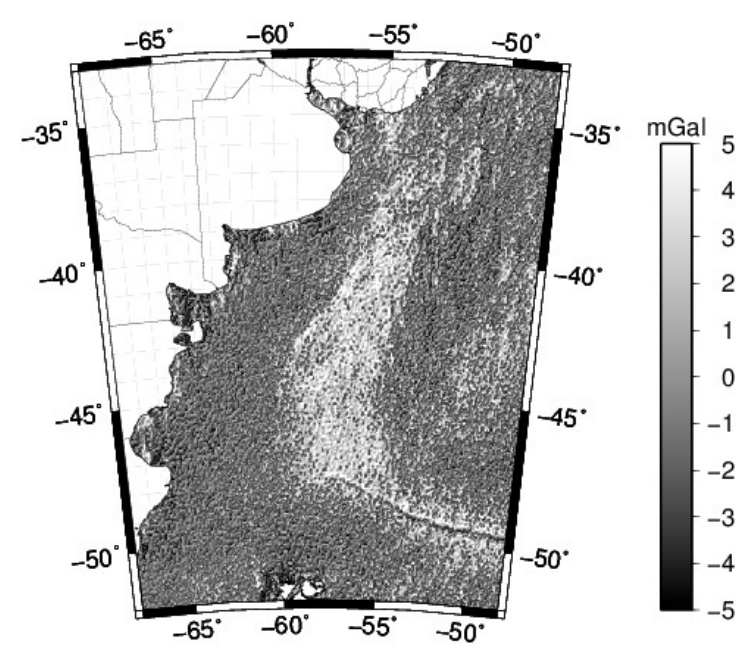

(b)

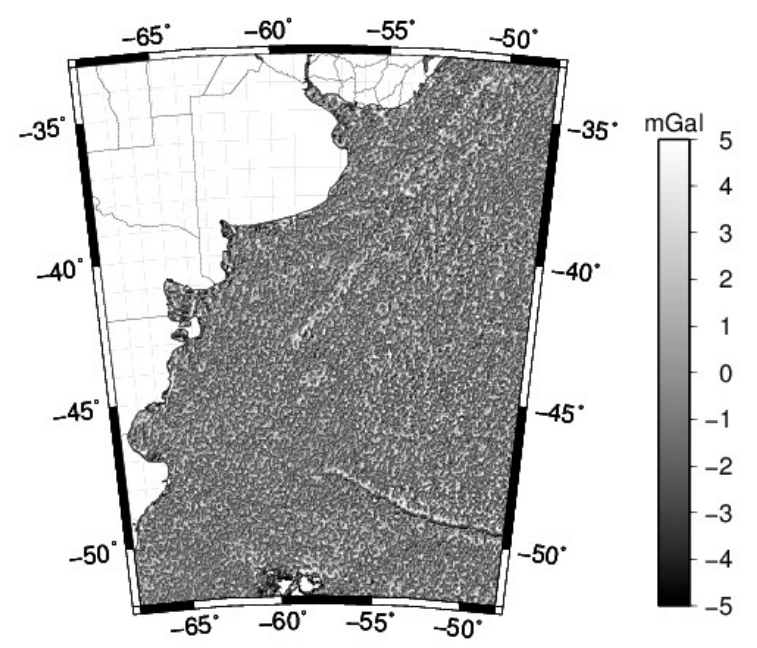

Figura 3.5. Diferencias entre grillas de anomalías de aire libre DNSC08SyS16.1 (a), izquierda y DNSC08-SyS18.1 (b), derecha.

son grillas de 1 minuto de arco por 1 minuto de arco; de ellas extrajimos datos para la región en las grillas SyS16.1, SyS18.1, DNSC08GRA Y DTU10GRA cuya estadística calculamos con la rutina grdinfo de GMT habiendo previamente excluido el modelo EGM96 o EGM2008 en la zona continental, según correspondiere. Los valores extremos de las diferencias entre las grillas DNSC08 y DTU10 vistos en la Tabla 3.2, están localizados en una región costera de la provincia de Chubut en las inmediaciones de Camarones y puerto Piojo.

En las Figuras 3.4a, 3.5a y 3.5b las estructuras residuales presentes en las diferencias se atenúan cada vez más, sin embargo persisten rasgos estructurales muy marcados en el área del margen continental cizallado y del talud, lo que indica una diferencia mas notoria de los modelos al mapear éstas zonas. En las Figuras 3.5b y 3.6a observamos una disminución del ruido aleatorio, pero las diferencias estructurales continúan existiendo.

Observando la Figura 3.6b, mapeándola con una escala de colores, es posible advertir que la diferencia entre los modelos DNSC08GRA y DTU10GRA muestra un desvío sistemático correlacionado latitudinalmente, que a grandes rasgos puede verse como una señal superpuesta de una longitud de onda de aproximadamente $3^{\circ}$ y amplitud de $2 \mathrm{mGal}$. El estudio de dicha señal escapa a los objetivos de éste análisis, pero sirve para identificar este desvío en alguno de los dos modelos o quizás en ambos que no 
(a)

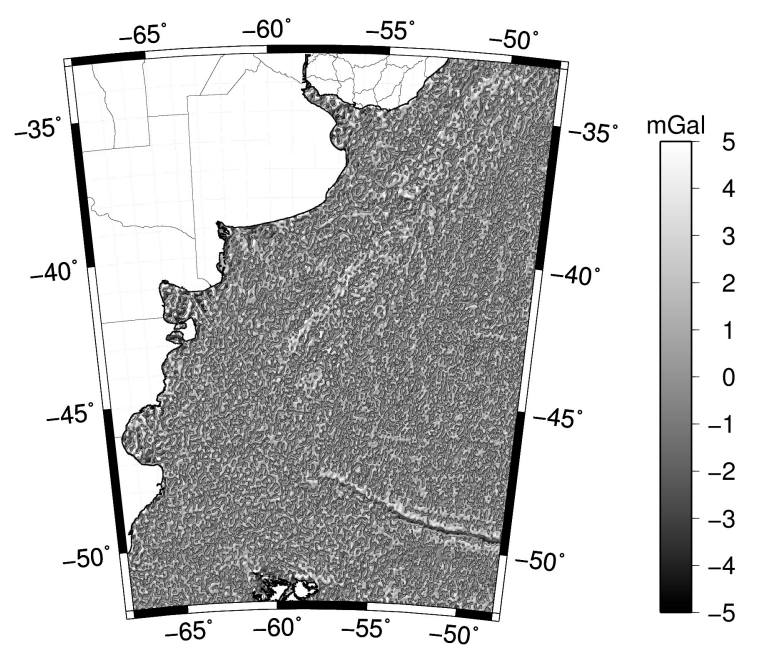

(b)

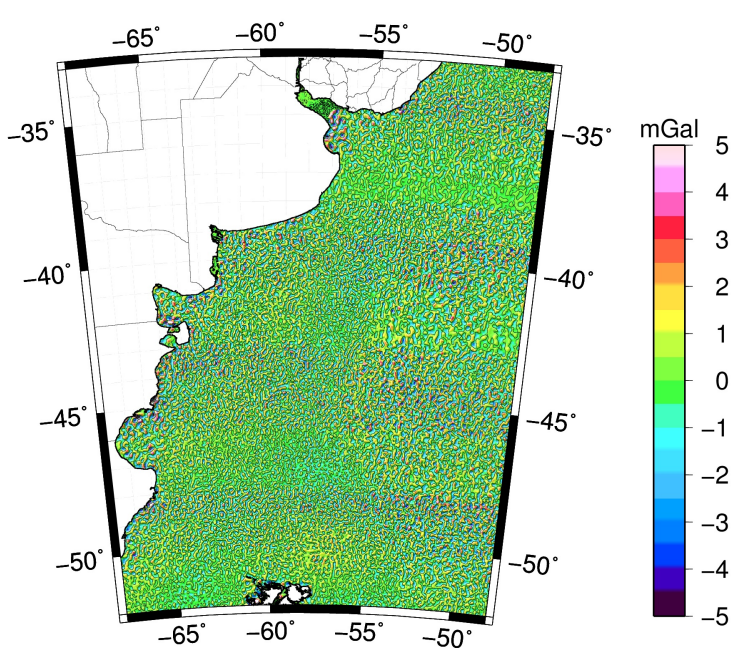

Figura 3.6. Diferencias entre las grillas DTU10-SyS18.1 (a), izquierda y DNSC08-DTU10 (b), derecha.

tiene características de una señal con información geofísica sino que es seguramente producto de un artificio matemático.

\subsubsection{Comparación entre anomalías de gravedad derivadas de altimetría satelital y anomalías de gravedad marina}

Interpolamos los datos de altimetría en la localización de las trazas de barco de las diferentes campañas con la rutina grdtrack de GMT con interpolación bilineal. Luego calculamos las estadísticas de las diferencias entre los valores de las derrotas de barco y los correspondientes interpolados en las grillas para las distintas campañas, las cuales observamos en las tablas 3.3 a 3.6, en la que mostramos la comparación entre las grillas de altimetría y los datos de barco de las campañas BGR87, BGR98-1, BGR98-2 y BGI, respectivamente. Para cada juego de comparación tenemos la estadística de las trazas individualizadas, lo que posibilita identificar aquellas que producen las mayores diferencias. Por ejemplo, una línea de la campaña BGI da diferencias del orden de $360 \mathrm{mGal}$ lo que es justificativo para su descarte antes de hacer la estadística de la tabla 3.6. Luego graficamos las diferencias en las líneas sobre el mapa de batimetría extraida del modelo DNSC08BAT [4].

En las Figuras 3.7 y 3.8 observamos las diferencias entre las anomalías de gravedad de aire libre de campañas marinas y las anomalías de gravedad derivadas de altimetría satelital. En la Figura 3.7 BGR87 (a) y BGR98-1 (b) con la grilla DNSC08. En la Figura 3.8, BGR98-2 (a) y BGI (b) con la grilla SyS18.1.

En las Figuras 3.7 y 3.8 observamos que los valores más altos de las diferencias se encuentran al principio o fin de una traza, lo que puede deberse a la influencia del movimiento del barco o a la automatización del sistema de registración y en puntos sobre el borde del talud. Dichos valores a veces son puntuales como el caso de las Figuras 3.7a y 3.8a y areales como en el caso de las Figuras 3.7b y 3.8b. 


\begin{tabular}{c|l|l|l|l}
\hline BGR87 N $^{\circ}$ p.: 10465 & \multicolumn{1}{|l|}{ máximo } & \multicolumn{1}{l|}{ mínimo } & media & \multicolumn{1}{c}{$\boldsymbol{\sigma}$} \\
\hline BGR87-SyS16.1 & 46.36 & -40.75 & 2.72 & 6.14 \\
BGR87-SyS18.1 & 43.27 & -44.20 & 1.23 & 5.73 \\
BGR87-DNSC08 & 43.75 & -42.45 & 1.20 & 5.72 \\
BGR87-DTU10 & 43.26 & -42.75 & 1.10 & 5.62 \\
\hline
\end{tabular}

Tabla 3.3. Estadística de diferencias entre anomalías de aire libre de la campaña BGR87 y derivadas de altimetría. Unidad: [mGal].

Las estadísticas de las diferencias están influenciadas por estos valores puntuales o areales de máxima diferencia. Consecuentemente, procedimos a trabajar en la edición de las trayectorias de los cruceros para detectar y eliminar errores groseros y/o de registración. Esto se hizo con la ayuda de la rutina mapproject de GMT, que calcula la distancia en kilómetros respecto del primer punto del perfil, lo que permite graficar en función de dicha distancia. Esto facilita el análisis individual, encontrar y eliminar valores erróneos manualmente.

En las Figuras 3.7b y 3.8b además los errores están asociados al hecho de ser mediciones muy próximas a la costa o en aguas someras donde los modelos de altimetría satelital continúan teniendo problemas dado que las técnicas de retracking no mejoran en este sentido [3] y la precisión de las anomalías de gravedad marina derivada de la altimetría no es la misma que en regiones del océano abierto. La razón de éstas discrepancias en zonas costeras se debe: 1) a que la topografía y la rugosidad del mar contaminan el frente de onda de los altímetros, 2) a que las mediciones altimétricas no pueden corregirse adecuadamente al existir inexactitud de las correcciones geofísicas de marea en aguas someras y retardo atmosférico, 3) a formas inapropiadas o imposibles de limpiar los datos de gravedad marina (análisis de cross-over y correcciones asociadas), 4) al hecho de no tener uniformidad en los datos en regiones costeras que es una necesidad al usar FFT ( gaps en las costas generan efectos de borde) [22].

El proceso de edición requiere una inspección visual para eliminar errores groseros y una minuciosa búsqueda de regiones ruidosas en las que debe evaluarse su eliminación con el compromiso de evitar la pérdida innecesaria de datos. Requiere entonces un tiempo dado que es manual y no es conveniente convertirlo en un proceso automatizado.

Realizamos nuevamente el cálculo de la comparación entre las anomalías derivadas de altimetría y la de las campañas editadas, mostramos los resultados en las tablas 3.7 a 3.10, en donde eliminamos líneas cortas y muy ruidosas.

Para hacer más explícita la información contenida en las tablas estadísticas 3.3 a 3.6 y 3.7 a 3.10 graficamos histogramas que se muestran en las Figuras 3.9 a 3.12. En estas figuras, la figura de la izquierda (a) representa el estado crudo de las diferencias, en las que se eliminaron derrotas con diferencias groseras, y en la figura de la derecha (b) el resultado luego de la edición de las trazas y su posterior comparación con la altimetría satelital. En las Figuras 3.9 y 3.10 mostramos la diferencia entre las campaña marina BGR87 y las grillas de altimetría satelital DNSC08 y SyS16.1, respectivamente. En las Figuras 3.11 y 3.12 mostramos las diferencias entre las campaña marina BGI y la grilla de altimetría satelital SyS16.1 y SyS18.1, respectivamente. 


\begin{tabular}{c|l|l|c|c}
\hline BGR98-1 N ${ }^{\circ}$ p.: 95566 & máximo & \multicolumn{1}{l|}{ mínimo } & media & \multicolumn{1}{c}{$\boldsymbol{\sigma}$} \\
\hline BGR98-1-SyS16.1 & 23.94 & -16.46 & 0.58 & 4.08 \\
BGR98-1-SyS18.1 & 23.17 & -19.62 & -0.81 & 3.67 \\
BGR98-1-DNSC08 & 20.42 & -20.37 & -0.83 & 3.76 \\
BGR98-1-DTU10 & 20.92 & -20.13 & -0.87 & 3.67 \\
\hline
\end{tabular}

Tabla 3.4. Estadística de diferencias entre anomalías de aire libre de la campaña BGR98-1 y derivadas de altimetría. Unidad: [mGal].

\begin{tabular}{|c|c|c|c|c|}
\hline BGR98-2 $\mathrm{N}^{\circ}$ p.: 76086 & máximo & mínimo & media & $\sigma$ \\
\hline BGR98-2-SyS16.1 & 21.71 & -14.70 & -0.34 & 3.68 \\
\hline BGR98-2-SyS18.1 & 19.53 & -17.37 & -2.45 & 3.21 \\
\hline BGR98-2-DNSC08 & 22.85 & -22.07 & -2.59 & 3.31 \\
\hline BGR98-2-DTU10 & 21.62 & -21.83 & -2.62 & 3.2 \\
\hline
\end{tabular}

Tabla 3.5. Estadística de diferencias entre anomalías de aire libre de la campaña BGR98-2 y derivadas de altimetría. Unidad: [mGal].

\begin{tabular}{c|l|l|l|l}
\hline BGI No p.: 7537 & \multicolumn{1}{l}{ máximo } & \multicolumn{1}{c}{ mínimo } & \multicolumn{1}{c}{ media } & $\boldsymbol{\sigma}$ \\
\hline BGI-SyS16.1 & 65.54 & -51.27 & 1.45 & 8.96 \\
BGI-SyS18.1 & 64.42 & -53.28 & 0.91 & 8.93 \\
BGI-DNSC08 & 65.45 & -53.22 & 0.91 & 8.98 \\
BGI-DTU10 & 65.04 & -50.90 & 0.90 & 8.94 \\
\hline
\end{tabular}

Tabla 3.6. Estadística de diferencias entre anomalías de aire libre de la campaña BGI y derivadas de altimetría. Unidad: [mGal].

(a)

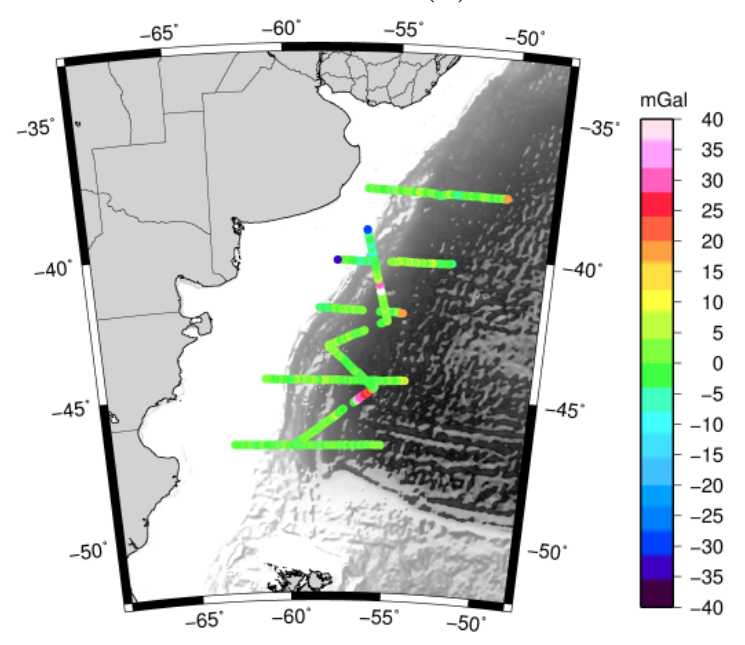

(b)

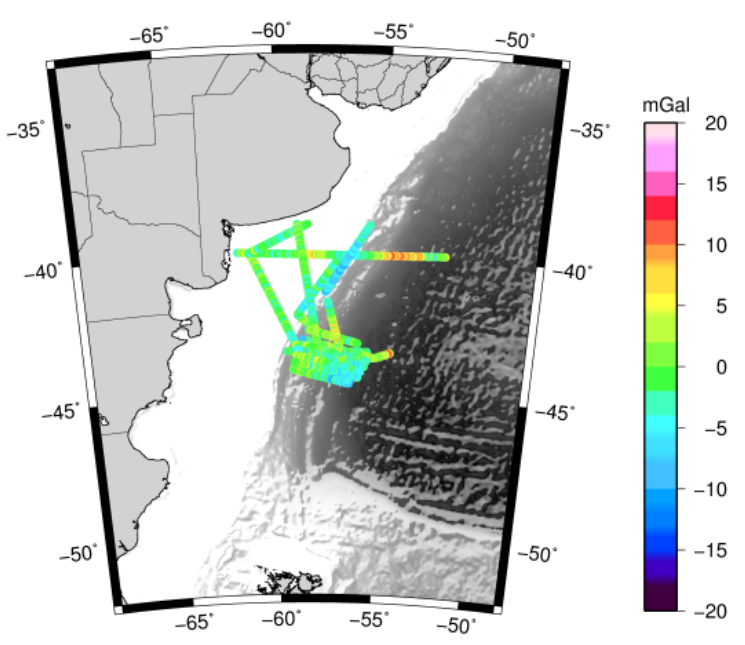

Figura 3.7. Diferencias entre anomalías de aire libre de (BGR87)(DNSC08) (a), izquierda y (BGR98-1)-(DNSC08) (b), sobre mapa de batimetría, derecha. 
(a)

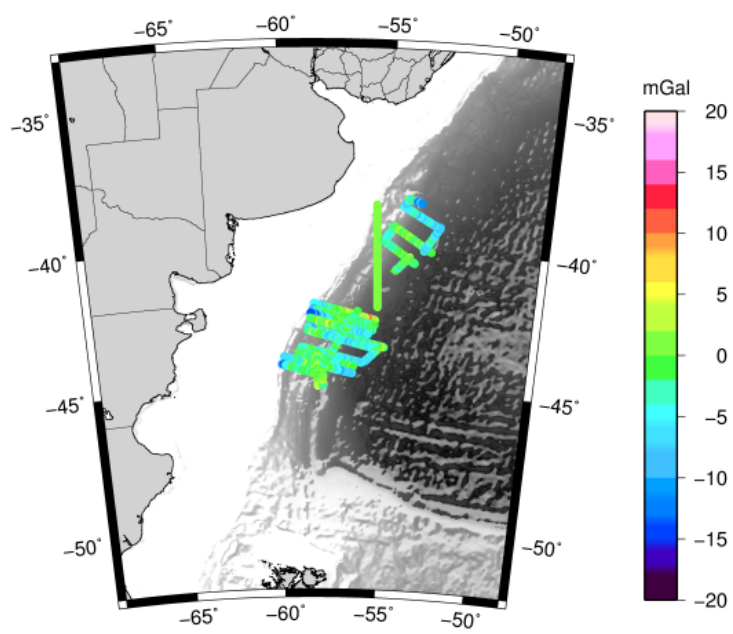

(b)

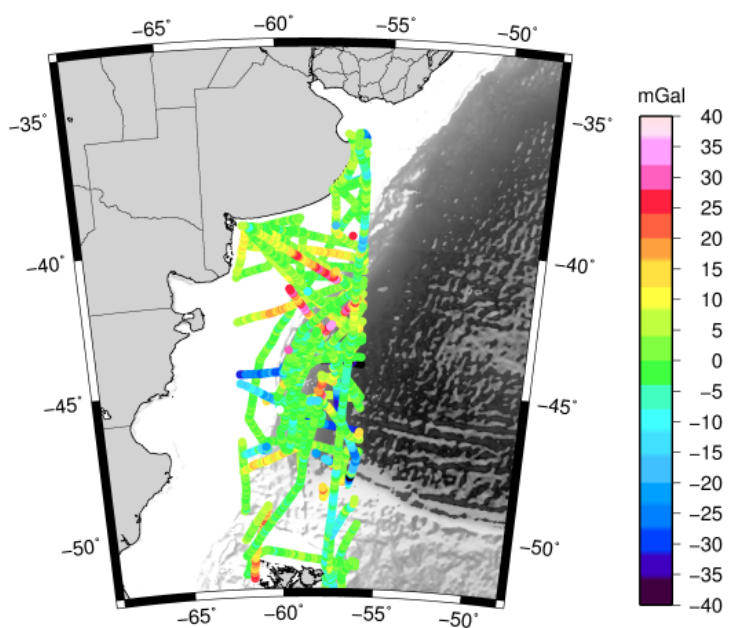

Figura 3.8. Diferencias entre anomalías de aire libre de (BGR98-2)(SyS18.1) (a), izquierda y (BGI)-(SyS18.1) (b), sobre mapa de batimetría, derecha.

\begin{tabular}{|c|c|c|c|c|}
\hline BGR87 $\mathrm{N}^{\circ}$ p.: 10114 & máximo & mínimo & media & $\sigma$ \\
\hline BGR87-SyS16.1 & 13.43 & -10.04 & 1.88 & 3.53 \\
\hline BGR87-SyS18.1 & 14.29 & -10.12 & 0.42 & 3.02 \\
\hline BGR87-DNSC08 & 15.63 & -9.64 & 0.40 & 3.03 \\
\hline BGR87-DTU10 & 12.95 & -10.18 & 0.30 & 2.87 \\
\hline
\end{tabular}

Tabla 3.7. Estadística de diferencias entre anomalías de aire libre de la campaña BGR87 editada y derivadas de altimetría. Unidad: [mGal].

\begin{tabular}{c|l|l|c|c}
\hline BGR98-1 N N $^{\circ}$ p.: 95418 & máximo & \multicolumn{1}{c}{ mínimo } & \multicolumn{1}{c}{ media } & \multicolumn{1}{c}{$\boldsymbol{\sigma}$} \\
\hline BGR98-1-SyS16.1 & 23.86 & -12.05 & 0.57 & 4.06 \\
BGR98-1-SyS18.1 & 23.17 & -12.24 & -0.81 & 3.66 \\
BGR98-1-DNSC08 & 20.42 & -12.24 & -0.85 & 3.74 \\
BGR98-1-DTU10 & 20.92 & -12.74 & -0.88 & 3.65 \\
\hline
\end{tabular}

Tabla 3.8. Estadística de diferencias entre anomalías de aire libre de la campaña BGR98-1 editada y derivadas de altimetría. Unidad: [mGal].

\begin{tabular}{c|l|l|l|l}
\hline BGR98-2 N N $^{\circ}$ p.: 75870 & máximo & \multicolumn{1}{l}{ mínimo } & \multicolumn{1}{l}{ media } & \multicolumn{1}{c}{$\boldsymbol{\sigma}$} \\
\hline BGR98-2-SyS16.1 & 16.29 & -14.70 & -0.34 & 3.66 \\
BGR98-2-SyS18.1 & 13.79 & -17.37 & -2.46 & 3.18 \\
BGR98-2-DNSC08 & 16.08 & -22.07 & -2.59 & 3.28 \\
BGR98-2-DTU10 & 14.88 & -21.83 & -2.62 & 3.17 \\
\hline
\end{tabular}

Tabla 3.9. Estadística de diferencias entre anomalías de aire libre de la campaña BGR98-2 editada y derivadas de altimetría. Unidad: [mGal]. 


\begin{tabular}{c|l|l|l|l}
\hline BGI N ${ }^{\circ}$ p.: 7080 & máximo & \multicolumn{1}{l|}{ mínimo } & \multicolumn{1}{l}{ media } & \multicolumn{1}{c}{$\boldsymbol{\sigma}$} \\
\hline BGI-SyS16.1 & 33.40 & -32.84 & 2.16 & 6.52 \\
BGI-SyS18.1 & 35.06 & -34.27 & 1.68 & 6.40 \\
BGI-DNSC08 & 36.14 & -35.05 & 1.67 & 6.44 \\
BGI-DTU10 & 36.22 & -34.85 & 1.65 & 6.40 \\
\hline
\end{tabular}

Tabla 3.10. Estadística de diferencias entre anomalías de aire libre de la campaña BGI editada y derivadas de altimetría. Unidad: [mGal].

(a)

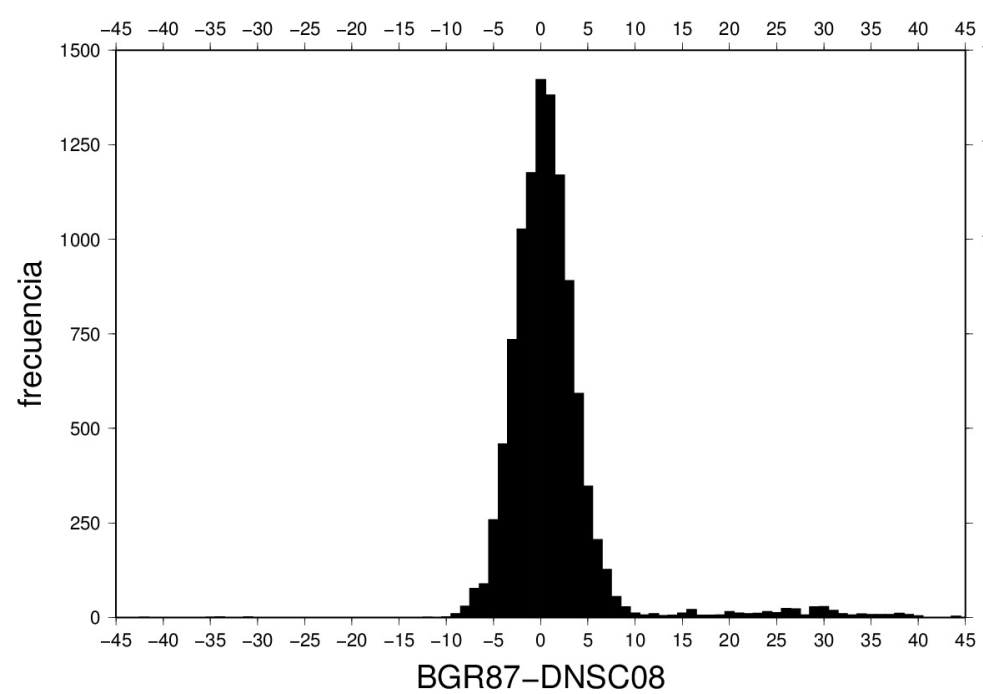

(b)

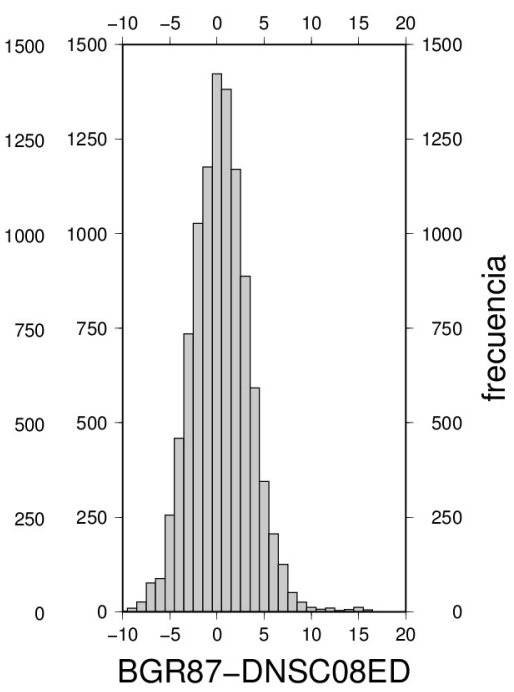

Figura 3.9. Histograma de las diferencias BGR87-DNSC08 crudo (a), izquierda y editado (b), derecha.

(a)

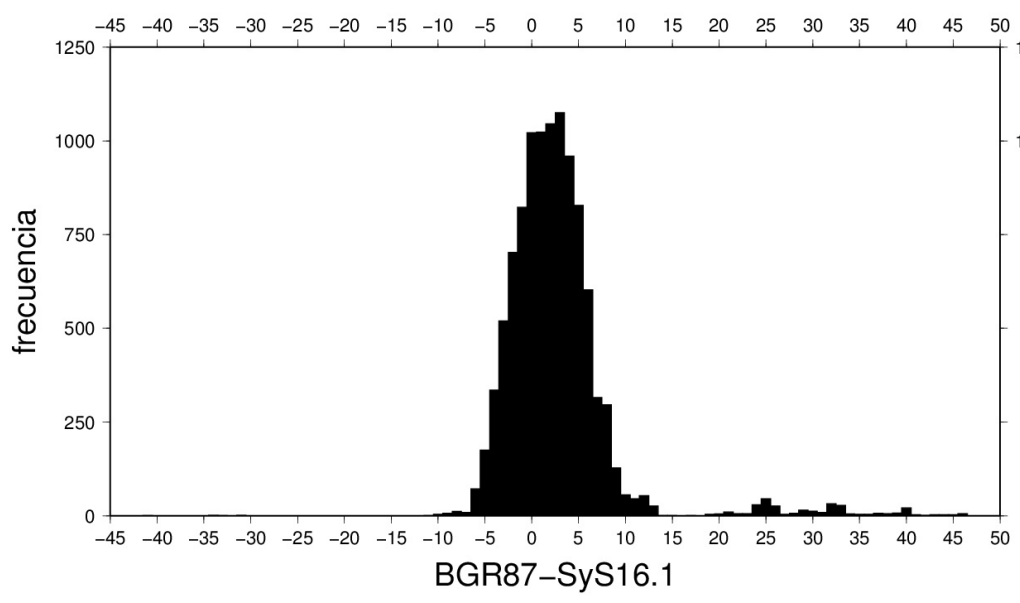

(b)

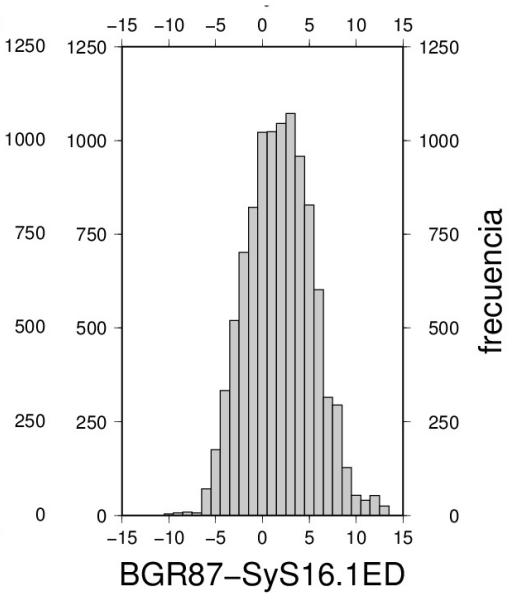

Figura 3.10. Histograma de las diferencias BGR87-SyS16.1 crudo (a), izquierda y editado (b), derecha. 
(a)

(b)
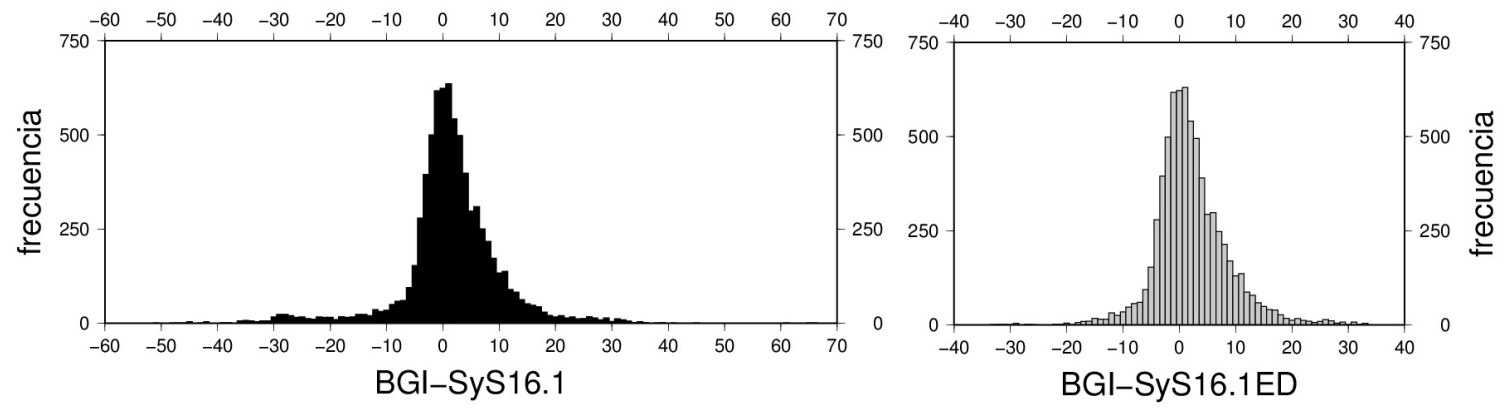

Figura 3.11. Histograma de las diferencias BGI-SyS16.1 crudo (a), izquierda y editado (b), derecha.

(a)

(b)
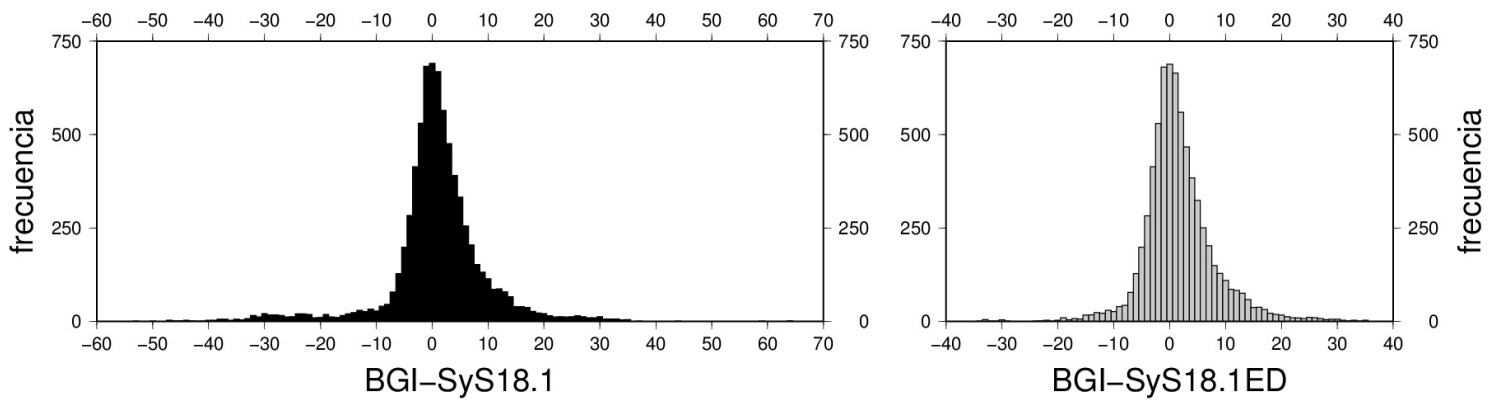

Figura 3.12. Histograma de las diferencias BGI-SyS18.1 crudo (a), izquierda y editado (b), derecha. 


\subsection{Conclusiones del capítulo 3}

De los análisis realizados se desprende:

1) Las mejoras introducidas en los modelos DNSCO8GRA y V18.1 hacen que éstos sean los más adecuados para su uso. Las ventajas de ambos modelos frente al modelo V16.1 se deben, entre otras, al uso de un mejor modelo geopotencial durante el procesamiento y principalmente, a los distintos procesamientos utilizados para su realización.

2)El nuevo modelo DTU10GRA mostró tener un mejor acuerdo al disminuir la media y desviación estándar de las diferencias entre campaña marina-altimetría satelital respecto al DNSC08GRA de casi todos los conjuntos de datos. Sin embargo existe un desvío sistemático correlacionado latitudinalmente en la diferencia entre ambos modelos.

3) En la comparación con anomalías de gravedad marina las diferencias más grandes son debidas a: a) las influencias del movimiento de rotación del barco en el inicio o finalización de una traza y b) la distribución de los datos marinos en la región del margen argentino en la zona del talud continental dado que la resolución de los modelos DNSO8GRA y V18.1 es buena en dicha región.

4) La utilización de datos de barco de diferentes fuentes requiere un análisis de la coherencia o consistencia de los mismos a través de una serie de pasos de depuración y posterior ajuste.

5) En primera instancia eliminamos líneas de datos de barco con errores muy groseros, de la comparación directa con los datos derivados de altimetría satelital. Luego se comienza una serie de pasos tendientes a mejorar la consistencia interna de las campañas, en los que el primer paso es la edición manual de los datos, paso que debe hacerse más exhaustivamente. Siguen pasos necesarios para practicar un ajuste por cruces o nivelación, proceso que aún no se ha realizado y se recomienda continuar.

6) Para los estudios que se harán en los capítulos siguientes se utilizarán las anomalías de aire libre derivadas de altimetría satelital de la versión V18.1 considerando que se desprende de la comparación hecha con las anomalías de barco que las diferencias son menores y aceptables. Teniendo en cuenta que las longitudes de onda que se usan en isostasia son largas, la longitud de onda es adecuada para el estudio de márgenes como el que se estudia en esta tesis y además por su cobertura que es más amplia y total.

\subsection{Propuesta para el análisis de cross-over}

La coherencia interna de cada campaña se mide a través del análisis de cruces (crossover), para lo que se buscan aquellas líneas que más puntos de cruce tengan por campaña para su reajuste, calculándose las diferencias de los valores de gravedad en esos puntos. Se propone realizar un ajuste polinómico por mínimos cuadrados que modele la variación del valor de anomalía de gravedad en el cruce en función de la distancia al primer punto de la línea seleccionada, con lo cual se mejoraría la coherencia interna de la campaña. Posteriormente se debería practicar el análisis de cruces entre campañas para mejorar la coherencia externa o cruzada. En cada paso se trataría de minimizar las diferencias con las anomalías de gravedad derivadas de altimetría satelital. Cuando haya finalizado todo este proceso la grilla que mejor ajusta en la región del margen continental argentino podría entonces combinarse con anomalías de gravedad marina en una base de datos unificada, dando mayor homogeneidad a los datos medidos desde 
plataformas a bordo de barcos para su utilización en diferentes aplicaciones. 


\section{Capítulo 4}

\section{Anomalías de Bouguer y la expansión de Parker}

En éste Capítulo comparamos dos metodologías de cálculo de la anomalía de Bouguer: La primera utiliza la relación entre topografía y gravedad con el desarrollo de Parker en el dominio de Fourier y la segunda calcula la anomalía de Bouguer completa (Bullard A, B o C) en varios rangos de distancias con diferentes aproximaciones para la atracción de prismas rectangulares y cónicos en las cercanías de la estación. Los resultados de ésta comparación fueron publicados en el trabajo de Pedraza De Marchi et al. [73].

\subsection{Comparación de anomalías de Bouguer}

La anomalía de aire libre como una medida de la variación de la masa de la Tierra constituye una aproximación de primer orden que determina el estado hidrostático de la misma. El estado hidrostático es el grado en el que varía la masa de la corteza (incluyendo los efectos de batimetría y topografía de lagos), que es compensado por cambios en profundidad de masas que llegan hasta el nivel en que la Tierra se halla en equilibrio hidrostático. Esta aproximación es la misma que la empleada para determinar el estado isostático que involucra no solo efectos topográficos sino variaciones de la densidad de la corteza. Es por esto, que en términos regionales, la anomalía de aire libre puede usarse para evaluar el estado isostático de la Tierra [51].

En contraste con la anomalía de aire libre, la anomalía de Bouguer tiene en cuenta el efecto de la componente gravitacional debida a la masa interpuesta entre el geoide y la estación de gravedad modelada. La anomalía de Bouguer se usa en modelado e interpretación geofísica de cuencas continentales y ambientes lacustres. Sin embargo, la mayoría de los trabajos sobre márgenes pasivos y océanos utilizan la anomalía de aire libre. La razón de esto es que la anomalía de Bouguer no es tan necesaria como lo es en las áreas continentales, donde los efectos topográficos normalmente enmascaran la contribución de las características de la corteza. No obstante aún existen intérpretes de los métodos que utilizan la anomalía de Bouguer en las regiones marítimas.

Hemos encontrado que en los estudios marinos que utilizan la anomalía de Bouguer, no se dice nada sobre el método empleado en el cálculo, posiblemente debido a que los autores creen que es sencillo y muy simple. Hemos encontrado que el cálculo de la anomalía de Bouguer en áreas marinas no es tan sencillo como se supone tácita- 
mente, y en consecuencia hemos experimentado en los métodos a utilizar. En lo que sigue describimos nuestra experimentación numérica que consiste en la comparación de dos metodologías diferentes para calcular las anomalías de Bouguer en el margen. El primero de ellos se basa en la expansión de Parker que tiene en cuenta los efectos topográficos y el segundo es el cálculo de la anomalía de Bouguer siguiendo una rigurosa metodología tradicional generalmente diseñada para las regiones continentales, pero que se ha extendido a las zonas marinas.

En áreas marinas la corrección de Bouguer implica reemplazar la densidad del agua por la de un material rocoso con densidad $2,67 \mathrm{~g} / \mathrm{cm}^{3}$. Hacer este reemplazo produce en aguas oceánicas profundas, grandes anomalías de gravedad positivas con un gradiente abrupto complicado para la interpretación. Esta es la razón por la que se usan anomalías de aire libre en el mar como más adecuadas para la interpretación [51].

En geofísica, la reducción de Bouguer se usa en un intento por remover los efectos gravitacionales de las masas topográficas. La estimación incorrecta de la densidad de las masas topográficas causa distorsiones en las anomalías de gravedad de Bouguer. Estas usualmente están altamente correlacionadas con las estructuras geológicas, causando problemas en las interpretaciones geológicas y aliasing en los datos grillados de gravedad de uso en geodesia [40, 42].

Hemos utilizado tres rutinas para obtener la anomalía de Bouguer: grdfft del paquete de herramientas de Generic Mapping Tools (GMT)[106], gravfft escrito por Joaquim Luis, que realizan operaciones matemáticas en el dominio de los números de onda, basados en la expansión de Parker, y la tercera es un programa Fortran 90 (FA2BOUG) desarrollado por Fullea et al. [34] con el que se obtiene la anomalía de Bouguer completa.

Si bien, aunque en FA2BOUG se ha refinado el método para el cálculo de la anomalía de Bouguer clásica, con gravfft hasta un cierto orden especificado obtenemos un cálculo adecuado para un cálculo a escala regional y la comparación revela que las diferencias entre FA2BOUG y grafft hasta cuarto orden sigue una distribución bimodal, cuya frecuencia máxima es de $1.8 \mathrm{mGal}$ y un desvío de $1.1 \mathrm{mGal}$ podríamos considerar a grandes rasgos que las diferencias están entre $1.8 \mathrm{mGal}$, lo cual se considera aceptable teniendo en cuenta la diversa metodología de cálculo. Con este resultado podemos decir que es confiable trabajar con las rutinas que están basadas en la transformada de Fourier y trabajan en el dominio de los números de onda para la interpretación de gravedad como nuestro objetivo general de entender la isostasia del margen y su área oceánica adyacente, entre otros estudios.

\subsubsection{Método 1, con la expansión de Parker}

El efecto de una topografía del fondo oceánico sin compensación (batimetría) al nivel del mar puede escribirse como [103]:

$$
\Delta g(k)=2 \pi G\left(\rho_{c}-\rho_{w}\right) H(k) e^{-k d}
$$

donde $\rho_{c}$ y $\rho_{w}$ son las densidades de la topografía del fondo y del agua, respectivamente. $H(k)$ es la transformada de Fourier de una topografía del fondo ondulante y $d$ es la profundidad media del fondo oceánico.

La ec. ( 4.1) asume que en el dominio de los números de onda $(k=2 \pi / \lambda)$, la anomalía 
de gravedad está linealmente relacionada con la batimetría.

La expresión completa para el efecto de gravedad de una topografía del fondo del mar sin compensación al nivel del mar es dada por la expresión de Parker [70]:

$$
F[\Delta g]=2 \pi G\left(\rho_{c}-\rho_{w}\right) e^{-k d} \sum_{n=1}^{\infty} \frac{k^{n-1}}{n !} F\left[h^{n}(x)\right]
$$

donde $h(\mathrm{~h}(\mathrm{x})$ o $\mathrm{h}(\mathrm{x}, \mathrm{y}))$ es la altura (positiva hacia arriba) respecto del valor medio $d$ de la superficie. La serie converge rápidamente si la máxima magnitud de h es mucho menor que d. $n$ es el orden. Cuando $n=1$ en la ecuación 4.2 se obtiene la ecuación 4.1 que es una aproximación de primer orden. Los términos de orden más altos están expresados en la ecuación 4.2. Mas adelante en el Capítulo 5 aparece un gráfico.

\section{Cálculo de la anomalía de Bouguer con el Método 1}

La anomalía de Bouguer se calcula con los siguientes pasos:

a) Usamos las rutinas grdfft(1 ${ }^{\text {er }}$ orden) y gravfft(ordenes mayores) de GMT (ver una descripcion de estas rutinas en el Apéndice C), para evaluar el efecto de gravedad de la interfaz agua-corteza $\left(w_{c}\right)$ calculada según las ecuaciones 4.1 y 4.2, respectivamente, gravfft está basada en la ecuación 4.2 hasta órdenes mayores.

b) Removemos los efectos de gravedad de la interfaz agua-corteza calculada en el paso a) de la anomalía de aire libre observada.

c) Calculamos la corrección de Bouguer como el efecto de una placa de contraste de densidad agua-corteza, de radio infinito y espesor igual a la altura media de la batimetría y la sumamos a la salida del paso b).

$$
\Delta g_{B} \cong \Delta g_{F A}-w_{c}+w_{c} p l a c a
$$

donde $\Delta g_{F A}$ es la anomalía de aire libre, $w_{c}$ es el efecto de gravedad de la interfaz agua-corteza calculada en el paso (a) y $w_{c}$ placa es el efecto de gravedad de una placa calculada en el paso (c).

\subsubsection{Método 2, anomalía de Bouguer completa}

En este método la anomalía de Bouguer se obtiene usando datos de anomalías de aire libre y de topografía o batimetría.

La corrección de Bouguer completa o topográfica históricamente se calcula en tres pasos: la corrección de la placa de Bouguer (Bullard A) que aproxima la topografía o batimetría local con una placa de extensión lateral infinita de densidad constante y espesor igual a la elevación del punto con respecto al nivel medio del mar, la corrección por curvatura (Bullard B) que reemplaza la placa de Bouguer por una capa esférica del mismo espesor y distancia superficial 166,735 km [52] y la corrección topográfica (Bullard C) que consiste en los efectos de la topografía circundante por encima y debajo de la estación. 


\section{Cálculo de la anomalía de Bouguer con el Método 2}

El programa Fortran FA2BOUG desarrollado por Fullea [34] calcula anomalía de Bouguer en tierra y en mar. El formato de entrada está adaptado para el uso de set de grillas globales de anomalía de aire libre y elevación. El programa calcula las correcciones Bullard A, B y C definiendo varias zonas que dependen de la distancia (R) horizontal al punto donde se calcula la anomalía de Bouguer y si el punto se encuentra en la tierra o en el mar. La batimetría es discretizada a través de una serie de prismas de tope plano centrados en cada punto de un modelo digital de terreno (DEM) y de altura la elevación dada por el DEM. El programa calcula directamente el efecto topográfico completo (placa de Bouguer corregida por curvatura más la corrección topográfica).

Para áreas marinas FA2BOUG define tres zonas de cálculo:

a) una zona interior $\left(R<\Delta x_{i} / 2 ; \Delta x_{i}\right.$ es el paso de la grilla):consiste en un cuadrado centrado en el punto de cálculo de lados $\Delta x_{i}$, en la que la elevación de cada vértice del cuadrado es interpolada linealmente utilizando los tres nodos vecinos más próximos y el punto de cálculo. La contribución de ésta zona se calcula combinando el efecto de un prisma de tope plano de altura correspondiente a la elevación en el punto y base la de la zona interna y de cuatro cuadrantes con pendiente constante desde cada vértice al punto de cálculo que contribuyen como cuartos en la atracción de un prisma cónico de eje vertical pasante por el punto de cálculo, para más detalles [34].

b) una zona intermedia $\left(R_{i}>R>\Delta x_{i} / 2\right)$ :

La atracción vertical producida por un prisma rectangular se calcula con la fórmula analítica en coordenadas cartesianas [65].

$R_{i}$ es el límite de la zona intermedia $(20 \mathrm{~km})$, elegido por una serie de tests que evalúan el compromiso entre precisión y el tiempo de cálculo [34].

c) una zona distante $\left(R_{d}>R>R_{i}\right)$ :

Se utiliza la expresión del desarrollo en armónicos esféricos del potencial para calcular la atracción vertical de un prisma rectangular [60] evitando inestabilidades numéricas En 1), 2) y 3) $R_{d}$ es el límite de la zona distante(167 km) y $\Delta x_{i}$ el paso de la grilla de la zona intermedia ( $2 \mathrm{~km}$ aproximadamente), el que se obtiene de la resolución de la grilla en minutos de arco $\left(1^{\prime}\right)$.

\subsubsection{Comparación entre ambos métodos}

El área de estudio corresponde a la sección del margen continental argentino entre las latitudes $39.5^{\circ} \mathrm{S}$ y $48.25^{\circ} \mathrm{S}$ y las longitudes $63^{\circ} \mathrm{W}$ y $51^{\circ} \mathrm{W}$. Para evitar efectos de borde que podrían surgir al trabajar con transformada de Fourier, consideramos un área de cálculo extendida 1 grado alrededor de ésta.

Los datos de anomalía de aire libre y batimetría provienen de las compilaciones globales V18.1 y V14.1, respectivamente de Sandwell y Smith [79]. La resolución de ambas versiones es $1^{\prime} \times 1^{\prime}$.

La Figura 4.1 muestra el mapa de anomalías de Bouguer completa usando a) FA2BOUG y b) gravfft hasta orden 4. Podemos observar la tendencia general de la correlación inversa entre la anomalía de gravedad de Bouguer y la batimetría. La anomalía de 
(a)

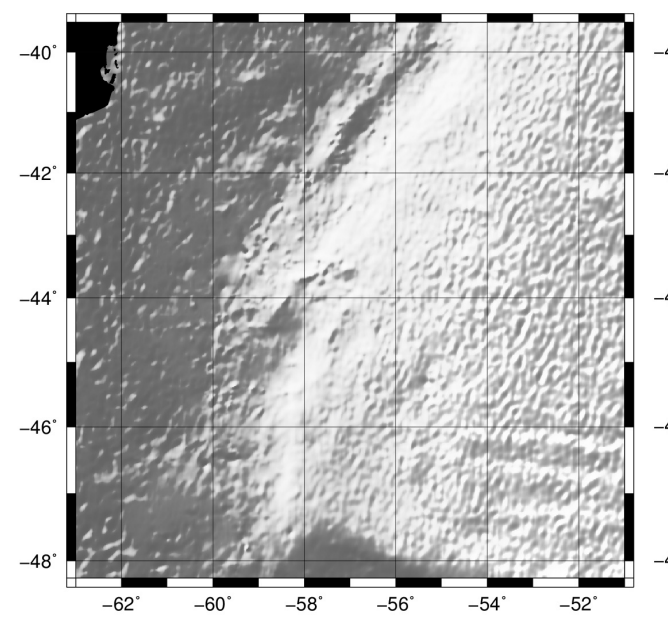

(b)

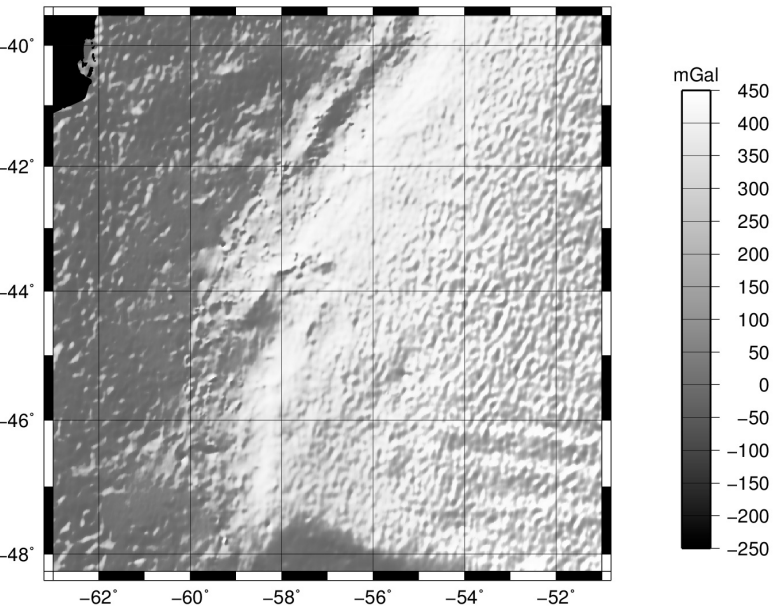

Figura 4.1. Mapa de anomalía de Bouguer completa usando el programa (a) FA2BOUG y (b) gravfft hasta órden 4.

\begin{tabular}{|c|c|c|c|c|c|c|}
\hline Diferencias & máximo & mínimo & media & mediana & moda & $\sigma$ \\
\hline FA2BOUG-grdfft & 21.23 & -14.30 & 0.27 & 0.35 & 0.52 & 2.27 \\
\hline FA2BOUG-gravfft $(\mathrm{n}=4)$ & 9.39 & -8.55 & 0.50 & 1.10 & 1.8 & 1.71 \\
\hline $\operatorname{gravfft}(\mathrm{n}=4)-g r d f f t$ & 17.24 & -12.99 & -0.23 & -0.32 & -1.18 & 1.62 \\
\hline $\operatorname{gravfft}(\mathrm{n}=5)-(\mathrm{n}=4)$ & 2.52 & -1.07 & $2.6 \mathrm{e}-5$ & $-1.5 \mathrm{e}-5$ & 0.00 & 0.04 \\
\hline
\end{tabular}

Tabla 4.1. Estadística de diferencias de anomalías de Bouguer calculadas con gravfft, grdfft, FA2BOUG y su comparación. Unidad: [mGal].

Bouguer es positiva en el océano atlántico y de gradiente muy abrupto en el área del talud, la cual cambia a lo largo del margen siguiendo esta estructura principal.

La Figura 4.2 muestra la comparación de anomalías de Bouguer calculadas usando el programa grdfft y gravfft hasta orden $\mathrm{n}=4$, respectivamente con FA2BOUG. En la Figura 4.3a podemos observar el comportamiento bimodal de la diferencia, que está caracterizado por un valor positivo en el área oceánica profunda y negativa en el área oceánica somera. Los mapas de la Figura 4.3 muestran las diferencias entre diferentes órdenes en la expansión de Parker. En a) podemos ver la diferencia entre $n=4$ y $n=1$ ( grdfft usa $\mathrm{n}=1$ ), que está fuertemente correlacionada con la topografía del fondo marino, mientras en $b)(n=5-n=4)$ observamos que las diferencias entre altos ordenes siguen correlacionadas con la topografía pero el efecto es menor, casi despreciable.

\section{Información estadística de las diferencias}

La estadística de la Tabla 4.1 sólo corresponde al área oceánica, hemos utilizado grdlandmask de GMT que mantiene los nodos de la grilla en la región marina. Analizando la información estadística de la Tabla 4.1, podemos concluir que incrementar el orden en la expansión de Parker más allá de $n=4$ no introduce cambios significativos respecto de las anomalías calculadas con FA2BOUG. Sin embargo podemos apreciar algunas diferencias espaciales entre los órdenes 4 y 5 con gravfft como puede observarse en la 
(a)

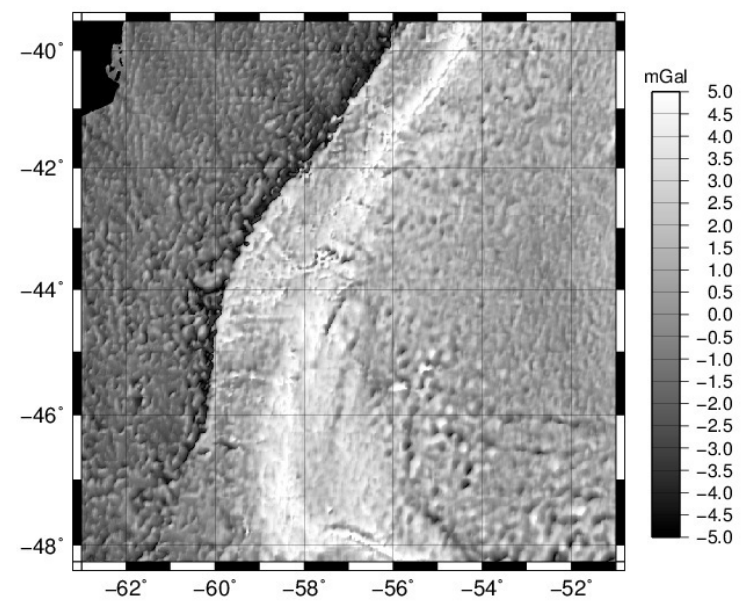

(b)

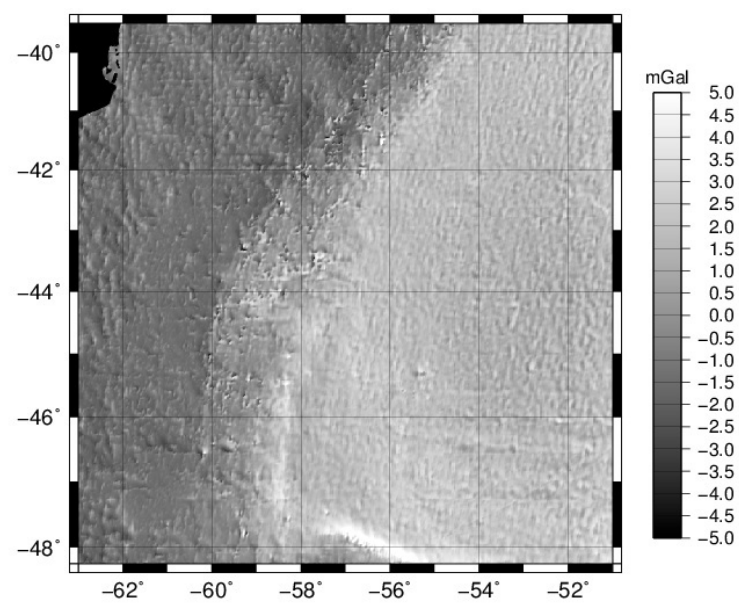

Figura 4.2. Diferencias (a) FA2BOUG y grdfft, (b)FA2BOUG y gravfft $n=4$.

(a)

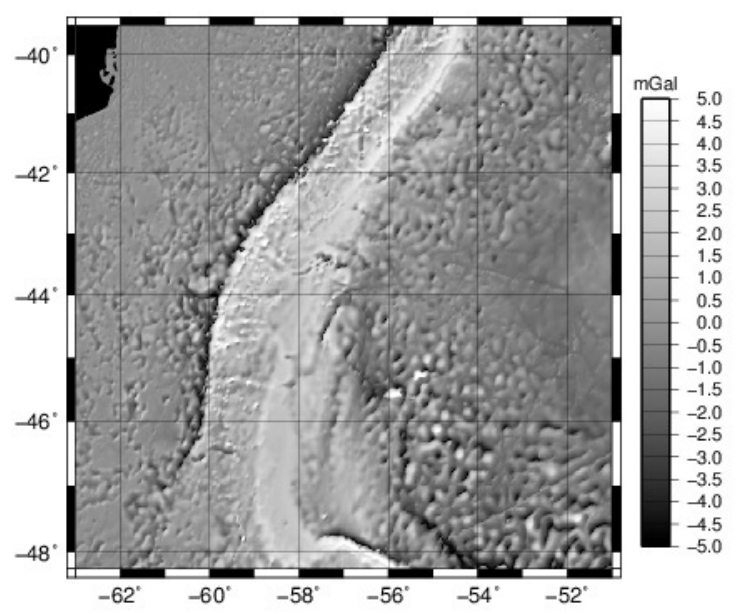

(b)

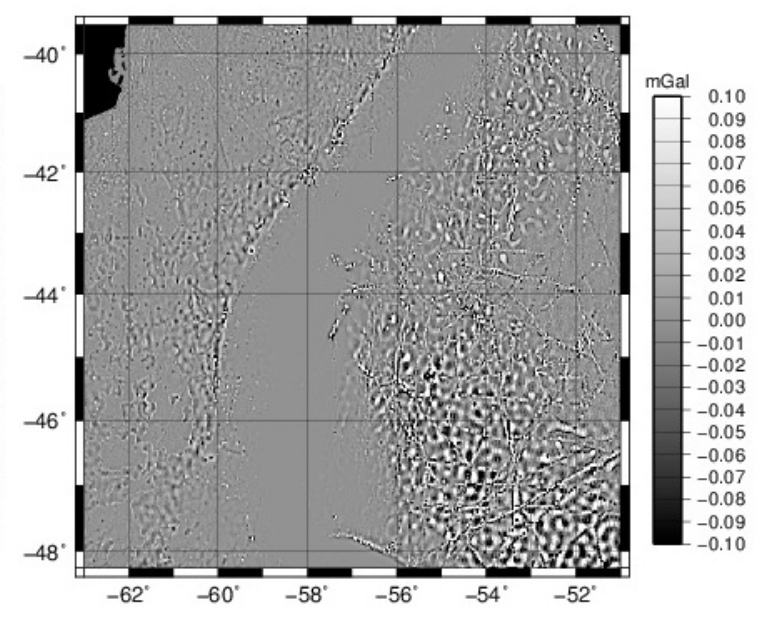

Figura 4.3. Diferencias (a) gravfft $\mathrm{n}=4$ y grdfft, (b) gravfft $\mathrm{n}=5$ y gravfft $\mathrm{n}=4$. 
Figura 4.3b y en la Tabla 4.1.

Dado que el parámetro media aritmética de un conjunto de datos es muy sensible a valores extremos, calculamos la moda y la mediana de las diferencias que son parámetros más adecuados para describir distribuciones de datos que no tienen una tendencia normal. La mediana muestra la simetría de la distribución en torno a la cantidad de datos, y la moda la diferencia para la cual la frecuencia es máxima. En las diferencias entre Parker de grado cuatro o mayor y Fullea la distribución es casi una bimodal (Figura 4.4a) y muestra un marcado sesgo, no así entre Parker de diferentes grados que resultan centradas y con un sesgo mucho menor (Figura 4.4b y Figura 4.5b).

$\mathrm{Al}$ tratarse de técnicas diferentes es aceptable que las diferencias más comunes se hallen entre $\pm 1.8 \mathrm{mGal}$ que podemos observar en la Tabla 4.1 y en la Figura 4.4a. Esta figura muestra dos frecuencias principales, que se encuentran alrededor de los valores $1.8 \mathrm{mGal}$ y $-1.5 \mathrm{mGal}$. La primera se asocia con la región de la llanura abisal (área océanica profunda), la segunda con los puntos de la terraza continental (área océanica somera) antes de cruzar el talud continental.

En la Figura 4.6 podemos observar que la diferencia tiene tres comportamientos principales: es positiva en el área oceánica profunda donde la frecuencia principal es de $1.8 \mathrm{mGal}$ (Fig. 4.6c), negativa en la zona oceánica somera con frecuencia principal de -1.5 mGal (Fig. 4.6a) y el resto se distribuye principalmente en la región del talud continental de los márgenes pasivo y cizallado (Fig. 4.6b).

Este comportamiento representa la principal desventajas de las rutinas gravfft y grdfft que están basadas en la expansión de Parker, dado que necesitan un valor promedio de la topografía del margen para poder aplicarse, y el margen tiene un cambio abrupto de topografía en la región del talud continental, que es la causa de la distribución bimodal. Podemos dividir el área en zonas y luego solaparlas en una única grilla, pero tendríamos que lidiar con cómo definir la zona del talud continental y como determinar cual el es mejor camino para solapar dichas zonas. Lo que representa una complicación numérica. Al hacer un cross plot de las diferencias para los cálculos usando gravfft con Parker de diferentes grados y FA2BOUG (Figura 4.8), se advierte que hay una dependencia no lineal de la diferencia con la topografía para diferencias en las que el grado en el desarrollo de Parker es menor que cuatro y aproximadamente lineal para grados más altos.

\section{Dos perfiles especiales de la anomalía de Bouguer}

Para testear localmente las diferencias vistas en las Figuras 4.2a y 4.2b y 4.3a, se toman una serie de perfiles longitudinales que se representan en función de la distancia al primer punto del perfil, donde las discrepancias entre las metodologías de cálculo son poco apreciables salvo casos puntuales donde se encuentra la máxima diferencia entre el método que usa el desarrollo de Parker de grado 4 y el de Fullea. Dos perfiles a la latitud $45^{\circ} \mathrm{S}$ y $48^{\circ} \mathrm{S}$, respectivamente se muestran en la Figura 4.7. La curva superior representa la anomalía de Bouguer calculada con los tres programas: FA2BOUG, grdfft, gravfft hasta orden $n=4$ y la curva inferior representa la correspondiente batimetría. Discrepancias entre éstas metodologías son despreciables excepto para los casos vistos en la Figura 4.7.

El área de la mayor discrepancia está generada por la contribución de la corta longitud de onda de la topografía del fondo oceánico como en el perfil de la Figura 4.7a para 
(a)

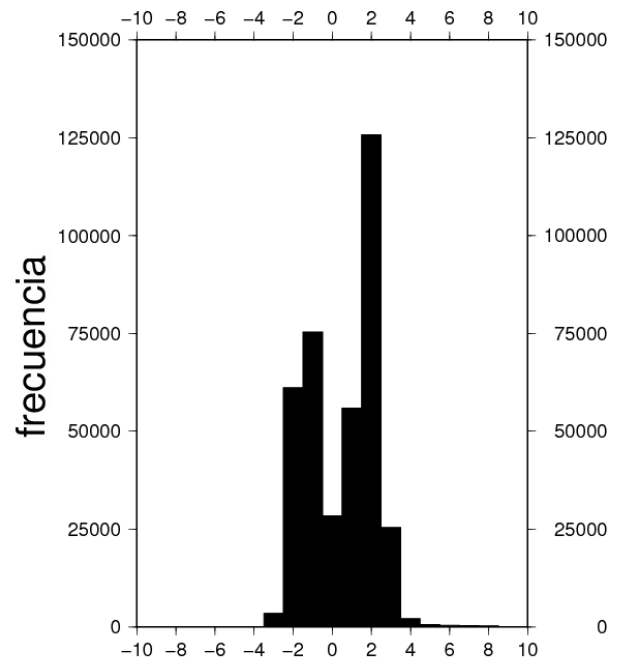

(b)

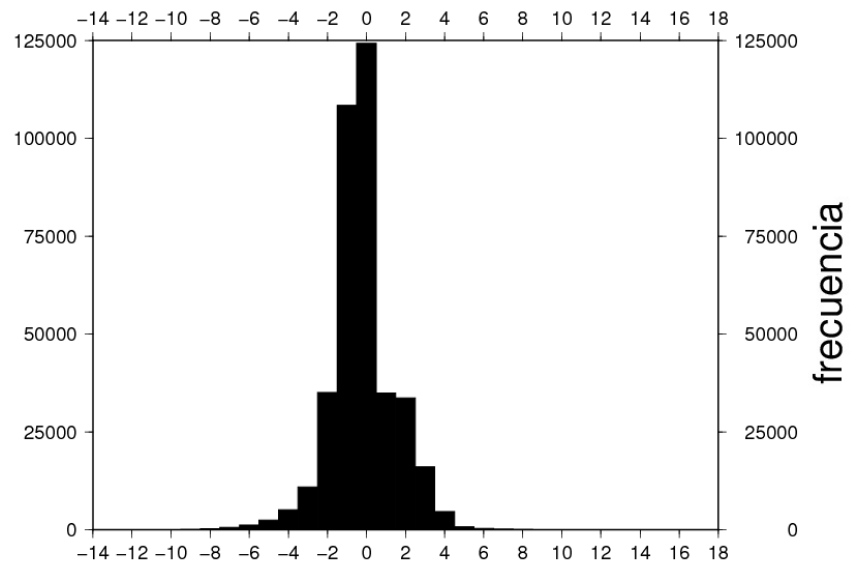

Figura 4.4. Diferencias (a) FA2BOUG menos gravfft $\mathrm{n}=4$, (b) gravfft $\mathrm{n}=4$ menos grdfft.

(a)

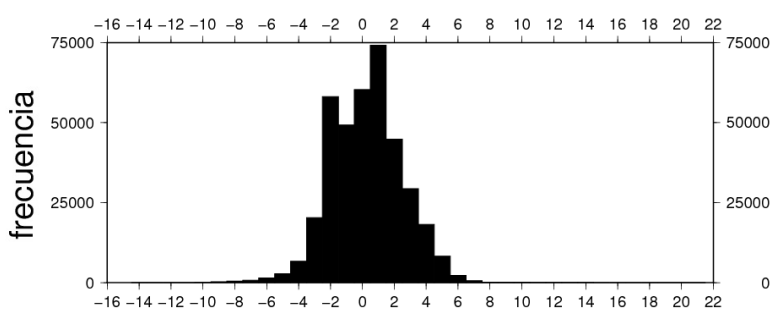

(b)

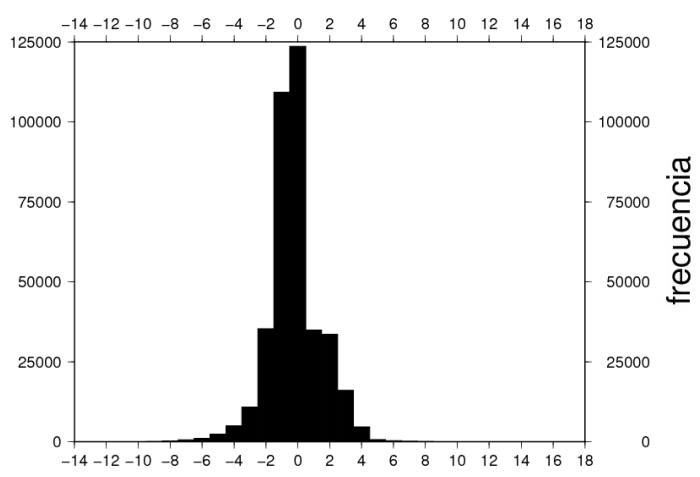

Figura 4.5. Diferencias (a) FA2BOUG menos grdfft, (b) gravfft $\mathrm{n}=5$ menos grdfft. 
(a)

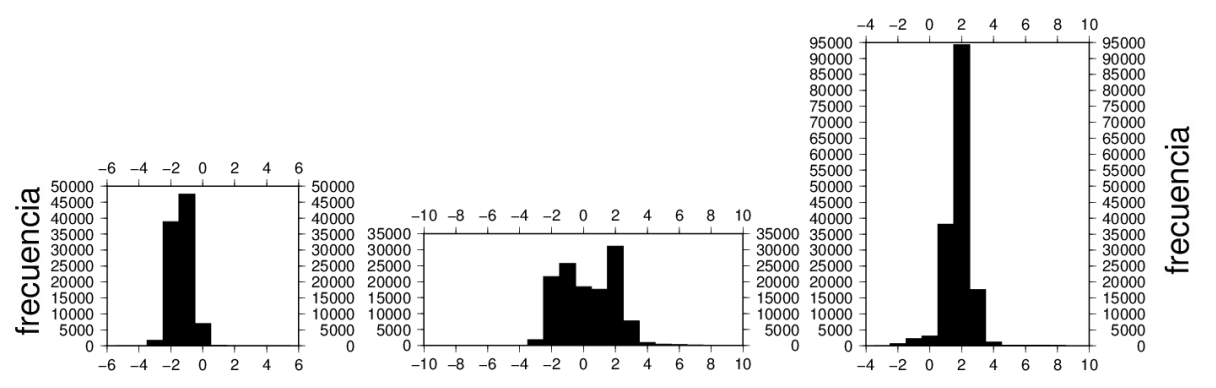

(c)

Figura 4.6. Diferencias FA2BOUG menos gravfft $\mathrm{n}=4$ en tres áreas longitudinales (a) $-63^{\circ} \leq \lambda \leq-60^{\circ}$, (b) $-60^{\circ} \leq \lambda \leq-56^{\circ}$, (c) $-56^{\circ} \leq \lambda \leq-51^{\circ}$ ( $\lambda$ es la longitud geográfica).

la latitud $45^{\circ} \mathrm{S}$, donde se halla un monte submarino de $1500 \mathrm{~m}$ de altura (aprox.) [27], el que causa la desviación de las curvas calculadas con grdfft respecto de las otras y las diferencias son de aproximadamente $20 \mathrm{mGal}$. (de acuerdo a la Tabla 4.1 y a la Figura 4.7a). Otras discrepancias podrían deberse a las inconsistencias en los datos del modelo de batimetría de Sandwell, pero en general, se deben a los cambios bruscos de pendiente de la topografía del fondo oceánico, es decir cambios muy abruptos de pendidente generan anomalías de Bouguer complicadas para su interpretación. Esta es la razón de que se usen anomalías de aire libre para la interpretación en zonas marinas [51]. En el caso de la Figura 4.7b, el perfil cruza un importante sistema de cañones submarinos condicionado por una activa dinámica de las masas de agua de origen Antártico, que se ha visto favorecido por un gran espesor de sedimentos conformando un sistema depositacional contornítico [27]. Podemos apreciar que la contribución de la corta longitud de onda de la topografía del fondo oceánico es pobre en el caso de la Figura $4.7 \mathrm{~b}$ aunque permite la diferenciación local de las tres curvas.

\section{Cross plot de las diferencias}

En promedio podría decirse que la diferencia de la anomalía entre Bouguer de grado $n=$ 4 y Fullea está linealmente correlacionada con la topografía (Fig. 4.8c). En la Figura 4.8 existen valores que no tienen un comportamiento polinómico ( Fig. 4.8a y 4.8b) o lineal (Fig. 4.8c). Para ver la distribución espacial de las máximas discrepancias se filtran los datos para las tres diferencias entre las metodologías de cálculo de la anomalía de Bouguer y se muestran en la Figura 4.9 y Figura 4.10 .

\section{Distribución espacial de los outliers}

Continuando con el análisis de las diferencias en las grillas de las Figuras 4.2 y 4.3, puede mostrase más adecuadamente que al aumentar el grado del desarrollo de Parker la independencia con la morfología del margen es mayor, las diferencias de más de 5 mGal en valor absoluto recorren la estructura del margen, es decir se ubican en su mayoría en el borde del talud (Figura 4.9b y Figura 4.10). Es de notar en la comparación entre 
(a)
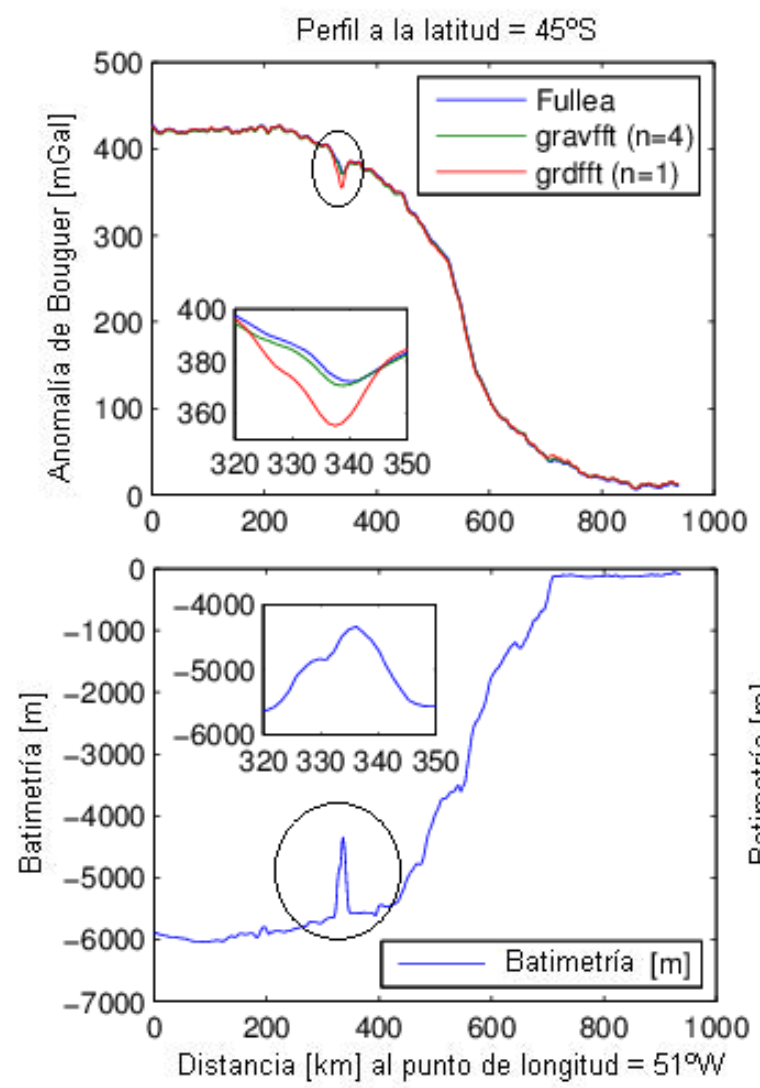

(b)
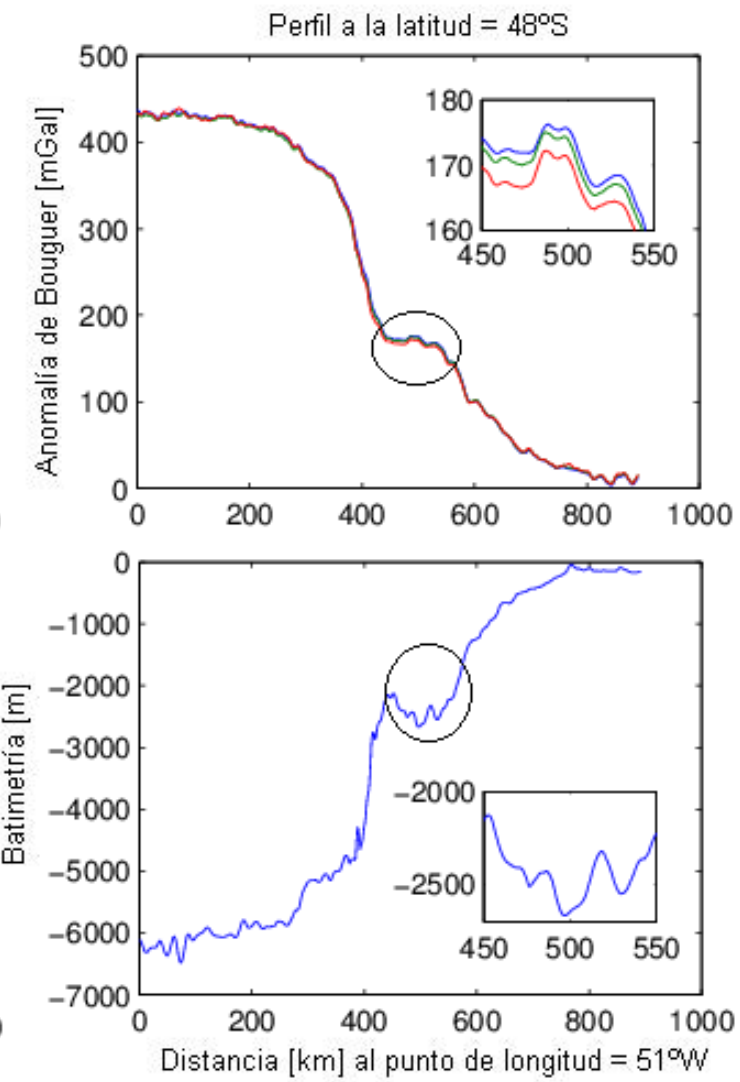

Figura 4.7. Perfiles longitudinales a la latitud (a) $45^{\circ} \mathrm{S}$, (b) $48^{\circ} \mathrm{S}$.

(a)

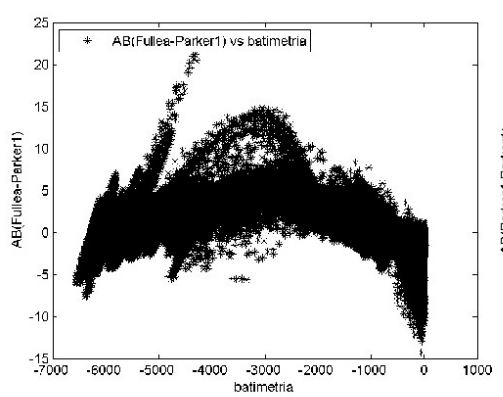

(b)

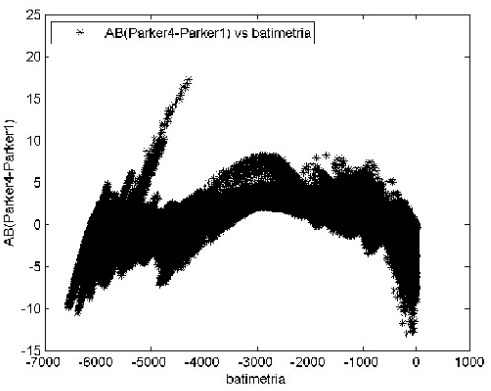

(c)

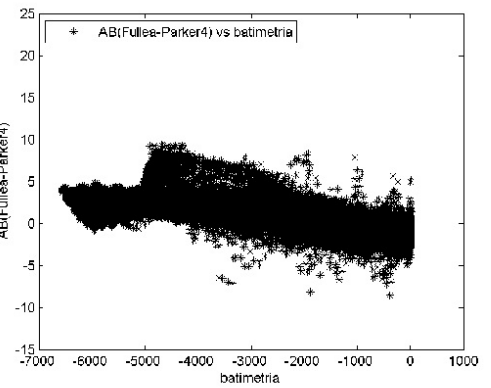

Figura 4.8. Cross plot de las diferencias Fullea-Parker1 (a), Parker4Parker1 (b) y Fullea-Parker4 (c), vs la batimetría. 
(a)

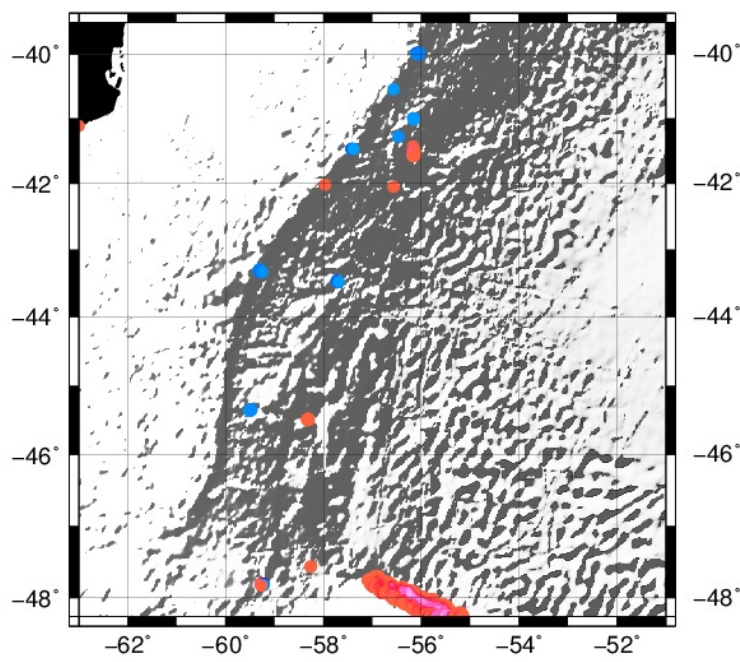

(b)

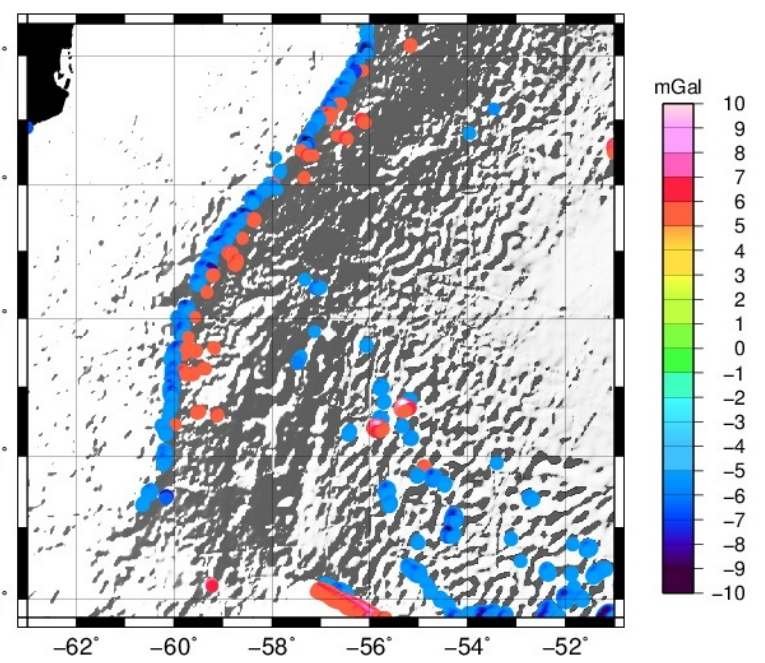

Figura 4.9. Diferencias Fullea-Parker4 $>$ 5mGal (a),Parker4-Parker1 (b) $>5 \mathrm{mGal}$.

los métodos de Fullea y el desarrollo de Parker de grado 4 que ambos son sensibles a estructuras como el del margen cizallado donde en la diferencia FA2BOUG-gravfft $\mathrm{n}=4$ ha disminuido el residuo positivo de la zona de dicho margen que se advierte en la Figura 4.10, lo que muestra que también Parker 4 es capaz de detectar esta estructura (Figura 4.9a ).

\subsection{Conclusiones del Capítulo 4}

Como la finalidad de lo desarrollado en este Capítulo es comparar los dos métodos, el que utiliza el desarrollo de Parker y el desarrollado por Fullea en su programa FA2BOUG, no se ha calculado una mejor aproximación a la anomalía de Bouguer, utilizando el método de Parker, que implicaría tener en cuenta una grilla de espesor sedimentario de la región y sumar su contribución a la anomalía de Bouguer con el contraste de densidad correspondiente. La metodología de cálculo propuesta en el programa de Fullea es rigurosa y podríamos llamarla robusta, sin embargo puede llegarse con mayor rapidez a un resultado similar utilizando transformada de Fourier con rutinas como grdfft o gravfft. La conclusión inmediata es que ambas metodologías resultan equivalentes en la tarea de aproximar la anomalía de Bouguer, y ambos métodos, el desarrollo de Parker a partir del grado cuatro y el de Fullea, son además sensibles a estructuras complejas como las del margen cizallado.

$\mathrm{Al}$ incrementar el grado de la expansión de Parker se obtiene una mejor aproximación de la anomalía de Bouguer, en la que hasta el grado 4 es visible la mejora respecto del cálculo usando el programa de Fullea.

Observamos en los perfiles que hay discrepancias entre los métodos en las regiones donde el talud continental presenta variaciones locales de muy alta frecuencia, regiones donde el talud continental es muy pronunciado y las variaciones bruscas de pendiente no pueden ser bien representadas. La diferencia entre las metodologías de cálculo de la 


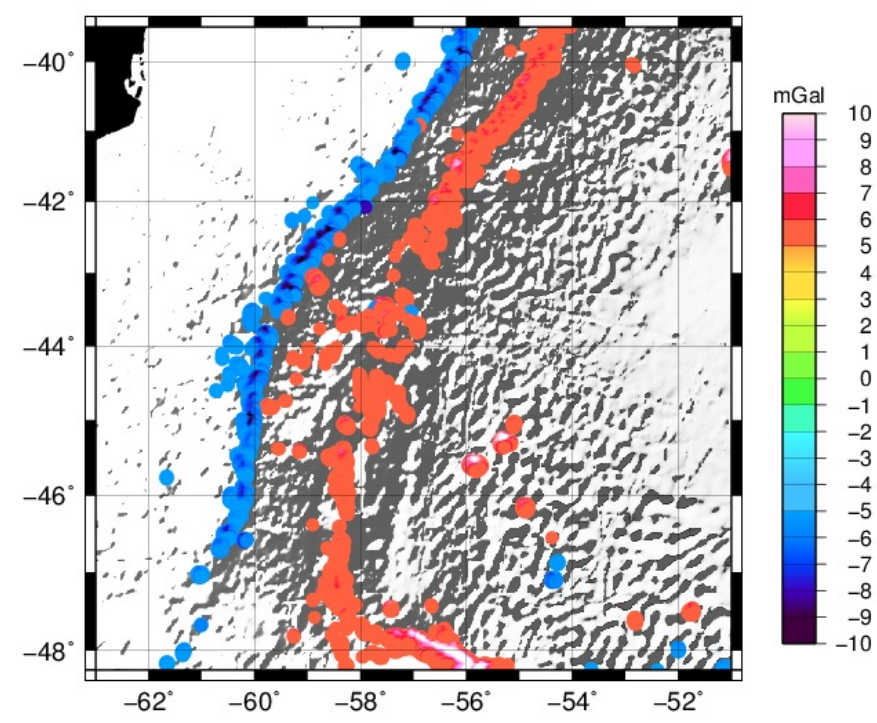

Figura 4.10. Diferencias Fullea-Parker1> 5mGal.

anomalía de Bouguer está en promedio altamente correlacionada con la topografía 


\section{Capítulo 5}

\section{Isostasia}

En este Capítulo presentamos una metodología de cálculo del efecto gravimétrico de tres interfaces con compensación isostática tipo Airy para una estimación de la anomalía isostática residual que refleja el estado de equilibrio isostático de la región de estudio. Luego se invierte la anomalía isostática para hallar la deflexión del Moho necesaria para compensarla. También se invierte la anomalía de Bouguer para hallar el Moho con un método iterativo y por último introducimos el concepto de isostasia flexural que será de utilidad en el Capítulo siguiente.

\subsection{Conceptos generales}

Isostasia es un concepto fundamental en las Ciencias de la Tierra. Se basa en la idea de una corteza ligera que flota en un manto más denso. La isostasia es un estado ideal: una condición en la que la corteza y el manto tenderán al reposo o equilibrio en ausencia de fuerzas perturbadoras. Algunos de los procesos que perturban la isostasia son: el avance y retroceso de las capas de hielo, la erosión, la sedimentación y el volcanismo extrusivo. La manera en que la corteza y el manto responden a estas perturbaciones condiciona las propiedades físicas de la litósfera a largo plazo y nos ayudan a comprender fenómenos complejos de la geodinámica como la generación de montañas, la formación de cuencas sedimentarias, la apertura de los continentes y la formación de nuevas cuencas oceánicas [103].

El concepto de isostasia describe la manera en que la superficie topográfica es compensada en profundidad. Estudios sísmicos muestran que el modelo de Airy explica satisfactoriamente variaciones en la estructura de la corteza asociadas con superficies como montañas y márgenes continentales.

La anomalía de aire libre como una medida de la variación de la masa de la Tierra constituye una aproximación de primer orden que determina el estado hidrostático de la misma. El estado hidrostático es el grado en el que varía la masa de la corteza (incluyendo los efectos de batimetría y topografía de lagos), que es compensado por cambios en profundidad de masas que llegan hasta el nivel en que la Tierra se halla en equilibrio hidrostático. Esta aproximación es la misma que la empleada para determinar el estado isostático que involucra no sólo efectos topográficos sino variaciones de la densidad de la corteza. Es por esto, que en términos regionales, la anomalía de aire libre puede usarse para evaluar el estado isostático de la Tierra [51]. 
Una de las características geofísicas más distintivas en márgenes continentales es el efecto de borde de la anomalía de aire libre. Este tiene la forma simple de un "alto" que se correlaciona con la parte exterior de la plataforma y un "bajo" asociado con el talud [102]. Tradicionalmente, el efecto de borde ha sido interpretado como el resultado de la yuxtaposición de corteza continental gruesa y corteza oceánica delgada que tienen distinta densidad por tener distinta composición. La anomalía puede explicarse por un modelo en el que la transición océano-continente se localiza en la región del talud en su ubicación actual. Sin embargo el efecto de borde es muy sensible a la localización de la transición, cambiando abruptamente si ésta se desplaza decenas de kilómetros tierra adentro o mar adentro de la zona del talud.

Uno de los objetivos de la isostasia es determinar las escalas temporales y espaciales sobre las que la corteza y el manto superior terrestres se ajustan a las cargas geológicas. Tales ajustes podemos determinarlos considerando de flexura en casos de lagos glaciares, montañas marinas e islas oceánicas, deltas de rio, sistemas de arcos de isla, operativo en escalas de tiempo de miles a pocos millones de años. El principal factor a determinar es el grado en que éstas superficies particulares están compensadas [103].

\subsection{Isostasia de Airy}

\subsubsection{Cálculo del efecto gravimétrico con tres interfaces y compensación isostática local}

El concepto de isostasia describe la manera en que la superficie topográfica está compensada en profundidad. Estudios sísmicos muestran que el modelo de Airy explica satisfactoriamente variaciones en la estructura de la corteza asociadas con superficies como montañas y márgenes continentales. El cálculo de la anomalía isostática se hace bajo la hipótesis de Airy de isostasia local, donde la compensación isostática es completa y local, es decir, la columna de masas está compensada directamente por debajo. Las densidades de la corteza y del manto se asumen constantes y se adopta un espesor normal de corteza.

La relación entre topografía del fondo oceánico y su efecto gravitacional, como ya se mencionó en el Capítulo 4 es [70]:

$$
F\left[\Delta_{g}\right]=2 \pi G\left(\rho_{c}-\rho_{w}\right) e^{-k d} \sum_{n=1}^{\infty} \frac{k^{n-1}}{n !} F\left[h^{n}(x)\right] .
$$

siendo $h$ la topografía respecto del valor medio $d$. Si la amplitud máxima de la topografía es mucho menor que la profundidad media del océano, entonces el primer término de la serie es dominante. Es común el uso de un sólo término, ya que provee una relación isotrópica lineal entre la trasformada de la anomalía de gravedad y la de la topografía, pero sólo es una aproximación.

$$
F[\Delta g]=2 \pi G\left(\rho_{c}-\rho_{w}\right) e^{-k d} F[h(x)] .
$$

La ecuación 5.1 es conocida como la fórmula de Parker que puede utilizarse para calcular el efecto gravimétrico de una interfaz arbitraria con el contraste de densidad correspondiente. Luego, podemos obtener la anomalía de gravedad a través del cálculo 


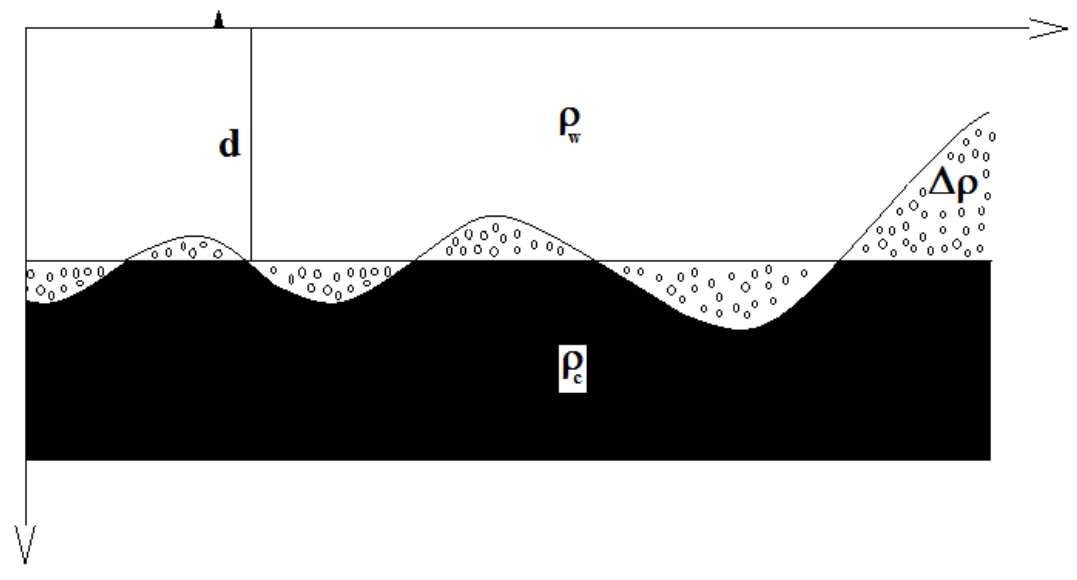

Figura 5.1. Interfaz agua-corteza ondulante respecto del valor medio de la batimetría.

de la suma de una serie de contribuciones consideradas. En esta tesis consideramos las interfaces agua-sedimentos, sedimentos-corteza y corteza-manto. Cada una de ellas es vista ondulante respecto de su valor medio, es decir la interfaz agua-sedimentos ondula respecto del valor medio de la batimetría, la interfaz sedimentos-corteza ondula respecto del valor medio de la profundidad del basamento y la interfaz corteza-manto ondula respecto del valor medio de la profundidad del Moho. Nos referiremos a ellas como esquema de tres interfaces, un ejemplo de una interfaz puede verse en la Figura 5.1.

En general, llamamos $C_{\text {Parker }}=2 \pi G\left(\rho_{1}-\rho_{2}\right)$ al producto de la ecuación 5.2, ya que $C_{\text {Parker }}$ es una variable que aparece frecuentemente en los cálculos de gravedad. Utilizando unidades en el sistema internacional $G$ es la constante de gravitación universal: $G=6,673210^{-11} \mathrm{Nm}^{2} / \mathrm{s}^{2}$. Al multiplicar por un espesor en metros, este factor da la atracción gravitatoria de una placa de ese espesor con densidad $\rho_{1}$ y sumergida en un medio de densidad $\rho_{2}$. La gravedad resultante está en $\mathrm{ms}^{-2}$. Para trabajar en unidades del $m$ Gal $\left(1 \mathrm{mGal}=1 \mathrm{~ms}^{-2} 10^{-5}\right)$ hay que multiplicar por $10^{5}$.

Con la ecuación 5.1 se puede calcular la gravedad generada por el relieve topográfico alrededor de un valor medio de profundidad, que se calcula con el signo adecuado.

Con la ecuación 5.1 se obtiene la gravedad generada por cada una de las interfaces: agua-sedimentos, sedimentos-corteza y corteza-manto en sus aproximaciones de primer orden utilizando la rutina grdfft $[106,107]$ de GMT y órdenes superiores con gravfft $[57,107]$.

\section{Cálculo de las topografías}

La batimetría, la profundidad del basamento y el Moho representan la referencia vertical para las interfaces agua-sedimentos, sedimentos-corteza y corteza manto, respectivamente. La profundidad del basamento se obtiene restando el espesor sedimentario (S) de la batimetría actual $\left(d_{w}\right)$. Cabe recordar que la batimetría es (-) y el espesor 


\section{Isostasia}

sedimentario es $(+)$. Para calcular el Moho hay que estimar el espesor cortical, para lo que se calcula la profundidad de la interfaz agua-sedimentos que habría si se quitaran los sedimentos considerando equilibrio isostático antes y después de la carga [103]. Sea una columna que inicialmente no está cargada de sedimentos (descargada), y luego se carga por sedimentos (cargada), ver Figura 5.2. Si la profundidad del agua $h$ está disponible para la sedimentación, el espesor final de los sedimentos debería ser $h$ más el incremento debido a la carga soportada por la corteza. Por lo que el espesor total de sedimentos a acumular es mayor que el del agua inicial. Luego, asumiendo equilibrio isostático antes (descargado) y después de la carga (cargado) respecto del nivel de compensación (NC), de acuerdo con la Figura 5.2 se obtiene:

$$
\begin{aligned}
d_{w_{0}} \rho_{w}+d_{c} \rho_{c}+w \rho_{m} & =\left(d_{w_{0}}-h\right) \rho_{w}+S \rho_{s}+d_{c} \rho_{c} \\
w \rho_{m} & =-h \rho_{w}+S \rho_{s} \\
w & =S-h
\end{aligned}
$$

De las ecuaciones 5.4 y 5.5 se obtiene la profundidad del agua $(h)$ que se necesitaría para explicar un espesor de sedimentos dado $(S)$ :

$$
\begin{gathered}
h=S \frac{\left(\rho_{m}-\rho_{s}\right)}{\left(\rho_{m}-\rho_{w}\right)} \\
d_{w_{0}}=d_{w}+S \frac{\left(\rho_{m}-\rho_{s}\right)}{\left(\rho_{m}-\rho_{w}\right)}
\end{gathered}
$$

El espesor de la capa de agua cuando no se tiene sedimentos $\left(d_{w_{0}}\right)$ es igual al espesor actual de la capa de agua con sedimentos $\left(d_{w}\right)$ más el producto del espesor sedimentario $S$ por el factor $\left(\rho_{m}-\rho_{s}\right) /\left(\rho_{m}-\rho_{w}\right)$ que representa el espesor de agua que se necesitaría para explicar dicho espesor de sedimentos.

Luego se calcula el espesor cortical sin sedimentos, balanceando un espesor de corteza de $T_{c}=32 \mathrm{~km}$.

$$
\begin{gathered}
d_{w_{0}} \rho_{w}+d_{c} \rho_{c}+w \rho_{m}=T_{c} \rho_{c} \\
d_{w_{0}}+d_{c}+w=T_{c}
\end{gathered}
$$

despejando $w$ de la ecuación 5.9 y reemplazando en la ecuación 5.8 se obtiene:

$$
\begin{gathered}
d_{w_{0}} \rho_{w}+d_{c} \rho_{c}+\left(T_{c}-d_{w_{0}}-d_{c}\right) \rho_{m}=T_{c} \rho_{c} \\
d_{w_{0}} \rho_{w}+d_{c} \rho_{c}+T_{c} \rho_{m}-d_{w_{0}} \rho_{m}-d_{c} \rho_{m}=T_{c} \rho_{c} \\
d_{w_{0}}\left(\rho_{w}-\rho_{m}\right)+d_{c}\left(\rho_{c}-\rho_{m}\right)=T_{c}\left(\rho_{c}-\rho_{m}\right)
\end{gathered}
$$

Despejando $d_{c}$ de la ecuación 5.12:

$$
d_{c}=T_{c}-\frac{\left(\rho_{m}-\rho_{w}\right)}{\left(\rho_{m}-\rho_{c}\right)}\left(-d_{w_{0}}\right)
$$

Finalmente el espesor cortical $\left(d_{c}\right)$ expresado como la suma del espesor normal de corteza $\left(T_{c}\right)$ más el producto del factor $-\left(\rho_{m}-\rho_{w}\right) /\left(\rho_{m}-\rho_{c}\right)$ por el espesor de la capa de agua sin sedimentos $\left(-d_{w_{0}}\right)$.

En las expresiones 5.7 y 5.13 se ha tenido en cuenta la convención de signos usada por 


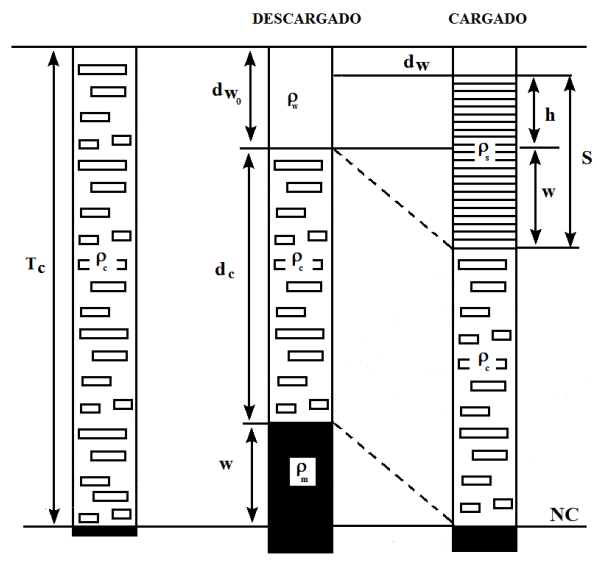

Figura 5.2. Equilibrio isostático antes (descargado) y después de la carga de sedimentos (cargado).

los datos en la que espesores son cantidades positivas y la topografía es positiva hacia arriba.

Una estimación de la profundidad del Moho puede obtenerse simplemente restando de la profundidad del basamento el espesor cortical $\left(d_{c}\right)$, llamamos a esta estimación Moho1.

Cálculo de las contribuciones de los efectos gravimétricos de las tres interfaces y anomalía isostática residual

Una vez calculadas las topografías de cada interfaz se obtienen las contribuciones de los efectos gravimétricos ws (agua-sedimentos), sc (sedimentos-corteza) y cm (cortezamanto) utilizando el desarrollo de Parker (ec. 5.1) hasta un cierto grado con los correspondientes contrastes de densidad y profundidad media de la interfaz. Luego puede estimarse la anomalía de aire libre como la suma de la contribución de las tres interfaces. La diferencia entre la anomalía de aire libre observada y la calculada puede atribuirse a la anomalía isostática residual.

$$
\Delta g_{I} \cong \Delta g_{A L}-(w s+s c+c m)
$$

donde $\Delta g_{I}$ es la anomalía isostática residual estimada, $\Delta g_{A L}$ es la anomalía de aire libre observada: $w s, s c$ y $\mathrm{cm}$ son las contribuciones a la anomalía de aire libre estimada $(w s+(s c+c m)$ de las interfaces agua-sedimentos, sedimentos-corteza y corteza-manto, respectivamente.

\subsubsection{Validación del método de cálculo de la anomalía gravi- métrica utilizando interfaces}

Con el fin de validar la metodología utilizada con el esquema de tres interfaces, pensamos en hacer la comparación de la técnica para el caso de usar sólo la interfaz agua-corteza y calcular la anomalía de Bouguer en el mar. Se trata de la interfaz más somera por lo que representa la contribución más crítica, dado que contiene la información de la corta longitud de onda. 


\section{Isostasia}

Como ya lo presentamos en el Capítulo anterior, calculamos la anomalía de Bouguer adaptando la metodología que utiliza el desarrollo de Parker con las rutinas grdfft o gravfft y la comparamos con el cálculo de la anomalía de Bouguer completa utilizando el programa FA2BOUG (correcciones Bullard A, B o C) [34]. La anomalía de Bouguer se calculó sumando una corrección a la contribución gravitacional de la interfaz agua-corteza (Fig. 5.1), la cual puede pensarse como una corrección topográfica del fondo oceánico, dado que la interfaz es descripta en función de su valor medio, donde se ha ignorado la presencia de sedimentos para la comparación. Es decir, la batimetría coincide con la profundidad del basamento o los sedimentos tienen igual densidad que la corteza. Calculamos la corrección de Bouguer como la atracción de una losa de contraste de densidad corteza-agua y de espesor igual a la altura media de la batimetría. Esta comparación ha sido desarrollada en el Capítulo anterior en el que se arribó a la conclusión que la diferencia entre el cálculo con el desarrollo de Parker de grado 4 y FA2BOUG presenta un comportamiento bimodal, es positivo en la zona oceánica profunda y es negativo en la zona oceánica somera, siendo la principal desventaja del uso de las rutinas basadas en el desarrollo de Parker la necesidad de un valor medio de la topografía para poder aplicarse y el margen tiene un cambio abrupto de topografía en el talud continental lo que produce la distribución bimodal de la diferencia. Con ésta comparación desarrollada en el Capítulo anterior hemos validado una metodología para calcular la anomalía de Bouguer que puede ser extendida al cálculo de la anomalía isostática.

\subsection{Aplicación}

\subsubsection{Espesor sedimentario}

La compilación de datos de espesor sedimentario es de varias fuentes, resultado de digitalizaciones de mapas publicados (http:// www.martagh.com.ar/mararg/pictr2002). La primera fuente de datos en las zonas marinas es de Ludwig et al. (1978) [56] y en las zonas terrestres valores de las Figuras de Zambrano y Urien (1970) [112]. La segunda fuente de datos es del Mapa Geológico de la República Argentina [12] que fue compilado en el Servicio de Hidrografía Naval [71]. La tercera fuente de datos de espesores se determinó digitalizando el mapa en la Figura 5 de Neben et al. (2002) [66]. En esa Figura, las isolíneas son de tiempo de ida y vuelta de la señal sísmica (TWT) en milisegundos, entre el fondo del mar y la discordancia de ruptura (breakup unconformity) o el basamento. Si se toma una velocidad media de $2000 \mathrm{Km} / \mathrm{s}$ para estimar aproximadamente los espesores, como en Hayes y LaBrecque (1991) [41], se obtiene un espesor sedimentario máximo de $4000 \mathrm{~m}$. Para nuestro mapa (Figura 5.3) se ha usado la ecuación que relaciona espesor sedimentario y tiempo de tránsito simple que se usó en Coscia (2000) [18]. Se trata de una ley semi empírica que surgió del análisis de cientos de soluciones de capas sedimentarias a profundidades variables en diferentes áreas del mar argentino. Coscia (2000) [18] estima que el error de determinación del espesor con esta fórmula es del 8\%. Los espesores así calculados varían entre 160 y 7540 metros. 


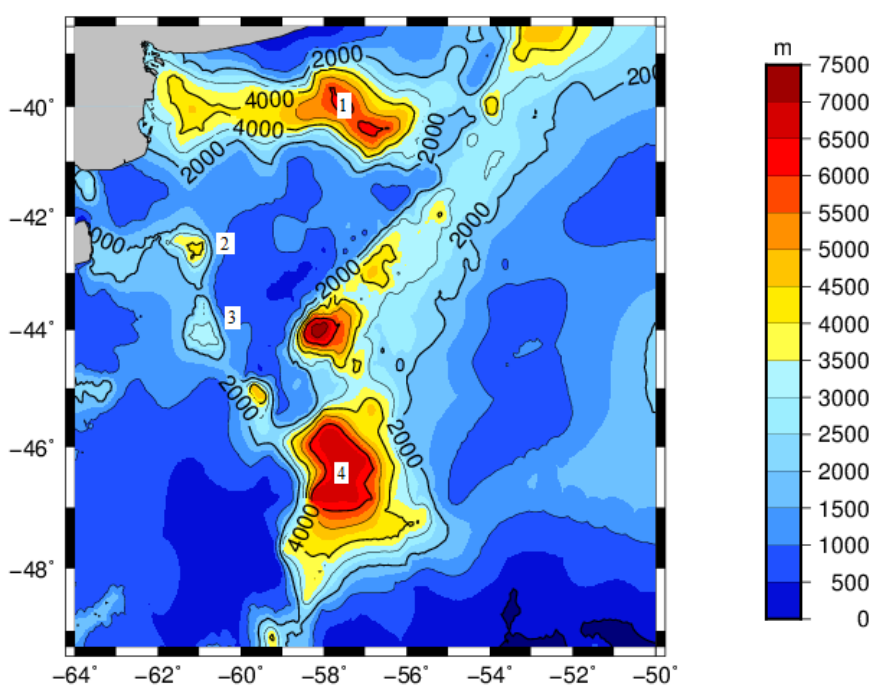

Figura 5.3. Espesor sedimentario. Se ha graficado la isobata de $500 \mathrm{~m}$, en color negro, para referencia. 1: cuenca del Colorado, 2: cuenca de Valdés, 3: cuenca de Rawson, 4: cuenca Argentina.

(a)

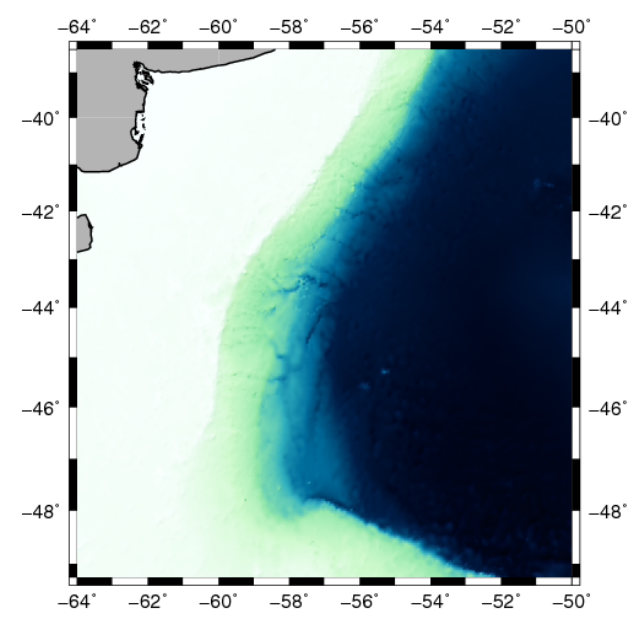

(b)

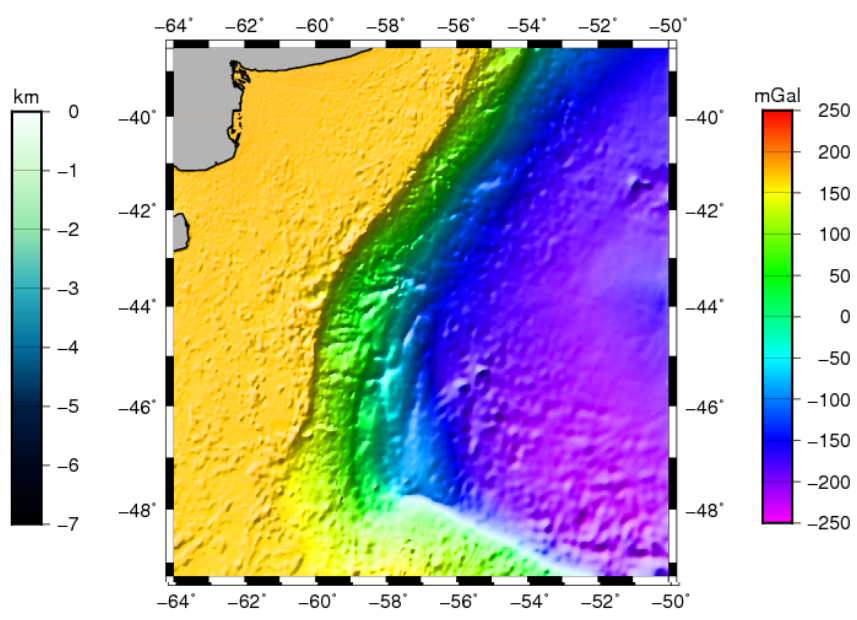

Figura 5.4. (a) Topografía (Batimetría) y (b) Contribución del efecto gravimétrico de la interfaz agua-sedimentos. 
(a)

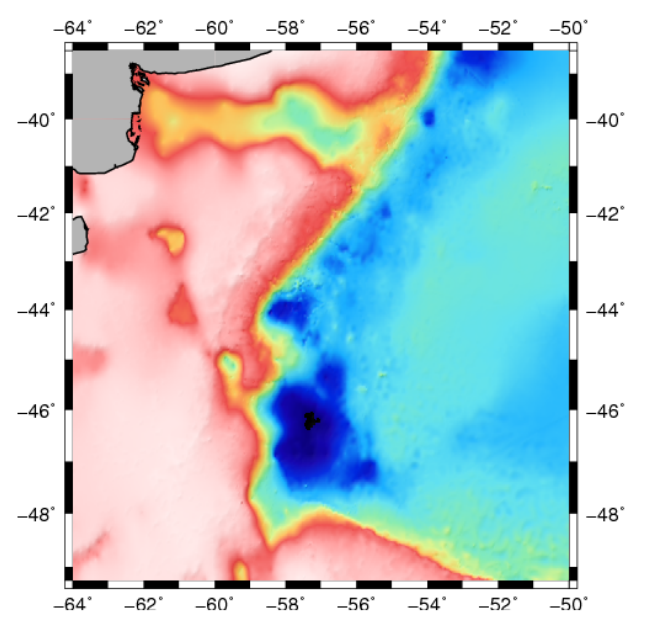

(b)

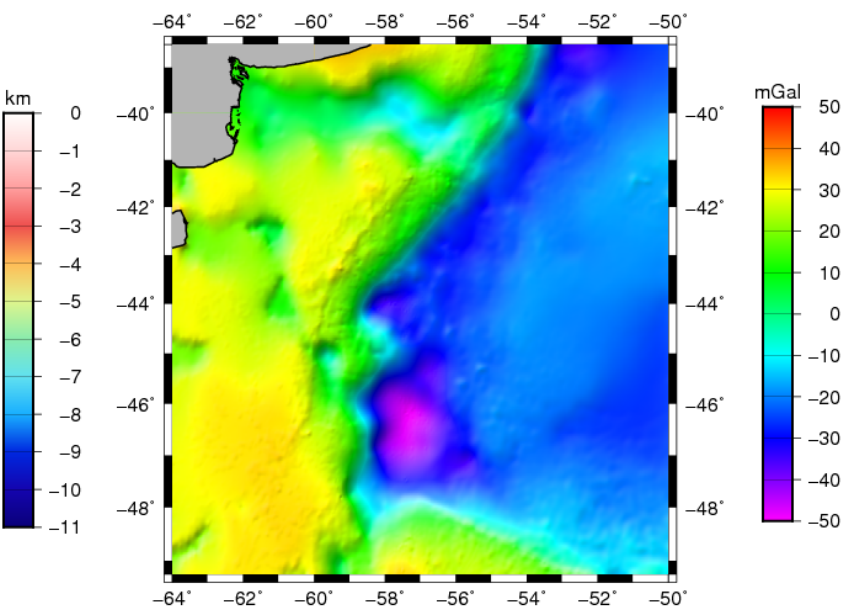

Figura 5.5. (a) Topografía (Profundidad del basamento) y (b) Contribución del efecto gravimétrico de la interfaz sedimentos-corteza.

(a)

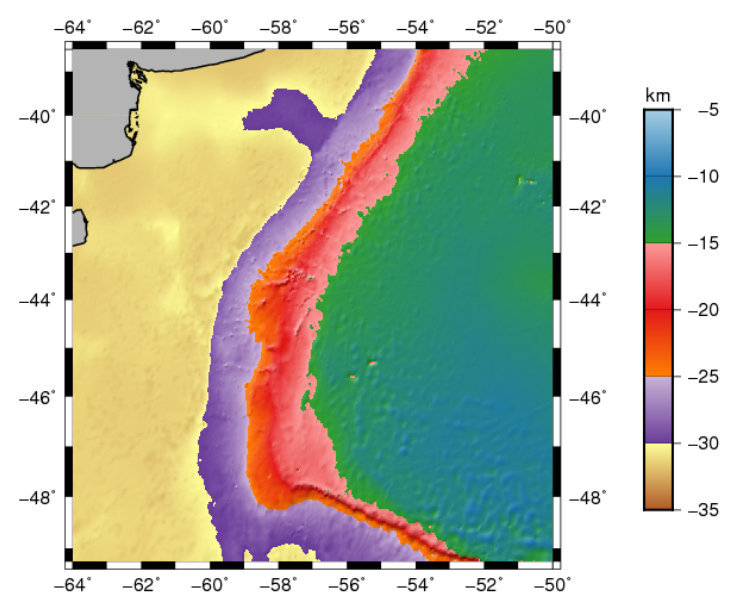

(b)

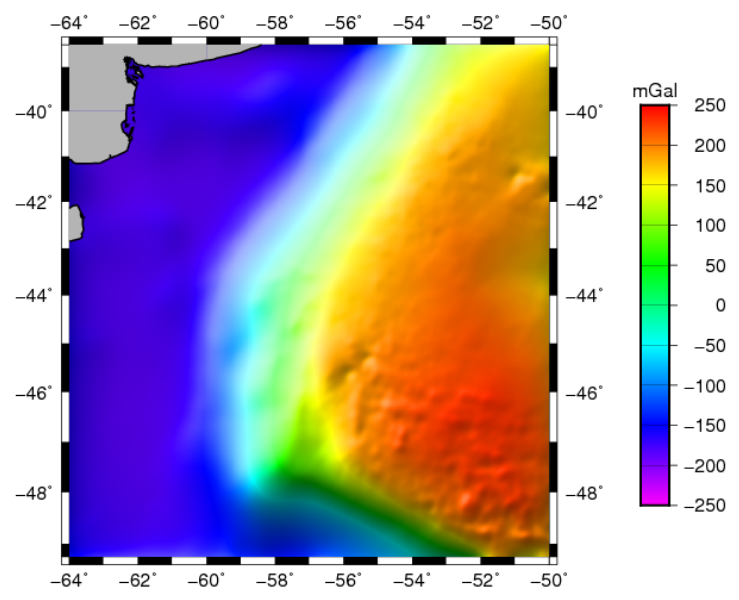

Figura 5.6. (a) Topografía (Moho) y (b) Contribución del efecto gravimétrico de la interfaz corteza-manto. 


\subsubsection{Análisis de densidades}

Para el presente trabajo de tesis se adoptaron los valores de densidad:

$\rho_{w}=1030 \mathrm{~kg} / \mathrm{m}^{3}$

$\rho_{s}=2600 \mathrm{~kg} / \mathrm{m}^{3}$

$\rho_{c}=2800 \mathrm{~kg} / \mathrm{m}^{3}$

$\rho_{m}=3330 \mathrm{~kg} / \mathrm{m}^{3}$

en los cuales el valor de la densidad de los sedimentos ha sido elegido a partir de la densidad de esta interfaz que minimiza la anomalía isostatica segun Airy calculada por Marta Ghidela con anterioridad al desarrollo de esta tesis.

\subsubsection{Topografía de las tres interfaces y sus contribuciones al efecto gravimétrico en la zona de estudio}

En las Figuras 5.4a, 5.5a y 5.6a se han graficado las topografías correspondientes a cada interfaz: batimetría, profundidad del basamento y Moho, respectivamente. Los efectos gravimétricos asociados a cada una de ellas (ws, sc y $\mathrm{cm}$ ) se presentan en las Figuras $5.4 \mathrm{~b}, 5.5 \mathrm{~b}$ y $5.6 \mathrm{~b}$, respectivamente. Puede observarse en las Figuras 5.4b, 5.5b y 5.6b que la contribución de la interfaz correspondiente es más suave mientras más profunda es la interfaz, como es de esperar, además se identifican los típicos efectos gravimétricos de la interfaz agua-sedimento (Fig. 5.4b) con un gradiente abrupto en la zona del talud y uno más suave de la interfaz corteza-manto (Fig. 5.6b) con grandes valores positivos en la zona marina profunda.

\subsubsection{Anomalía isostática residual y la influencia de los sedi- mentos en su cálculo}

Conociendo las limitaciones de aplicar el método basado en el desarrollo de Parker en la zona de estudio desarrolladas en el Capítulo 4, presentamos la anomalía isostática calculada con la expansión de Parker de grado 4 (ec. 5.1) utilizando la rutina gravfft de Joaquim Luis [57] que se observa en la Figura 5.7b. En la Figura 5.7a se ha graficado la anomalía de aire libre, considerada una anomalía isostática a primer orden. Notar que la isobata de $500 \mathrm{~m}$ coincide con la ubicación del efecto de borde típico en casi toda el área.

En la Figura 5.7b la anomalía isostática según Airy presenta una zona de valores negativos en la zona del talud, bordeando la isobata de $500 \mathrm{~m}$, en algunos lugares a veces muy intensos. Es en esta zona donde el análisis de datos sísmicos descriptos en el texto muestra la presencia de grabens en el basamento. En la cuenca del Colorado aparecen anomalías positivas que pueden indicar que la cuenca estaría en subsidencia. Las cuencas de Valdés y de Rawson se ven como señales positivas débiles. Justo en la zona donde las características del margen cambian aparecen un par de anomalías, una de ellas de forma casi circular negativa $\left(45^{\circ} \mathrm{S}, 59^{\circ} \mathrm{W}\right)$ y la otra triangular positiva $\left(44^{\circ} \mathrm{S}, 58^{\circ} \mathrm{W}\right)$ separadas aproximadamente por la zona de la discontinuidad del Colorado. Comparando con el mapa de espesor sedimentario la zona positiva coincide con un máximo (cuenca Argentina), que por ahora tentativamente podría interpretarse como una cuenca en subsidencia, pero merece realizarse un análisis más exhaustivo. Podría 


\section{Isostasia}

también ocurrir que en ese lugar hubiera un alto en el basamento o también podría tratarse de una intrusión en profundidad de material más denso.

Si observamos la Figura 5.7b junto a la Figura 5.3, se evidencia una correlación entre las zonas de máximo espesor sedimentario y los máximos de la anomalía isostática, asociados a la incorporación en el cálculo de la anomalía isostática de la contribución de la interfaz sedimentos-corteza. Esta observación nos hace pensar acerca de cuál es el papel que la inclusión de los sedimentos tiene en el cálculo de dicha anomalía.

Si calculamos un cross plot de la diferencia del cálculo de la anomalía isostática no teniendo en cuenta la presencia de los sedimentos y considerándolos versus el espesor sedimentario, confirmamos que existe una fuerte correlación entre ambas series de datos, cuyo coeficiente de correlación (ce) es de -0.7597 en promedio para toda la zona, lo cual es muy representativo. Si calculamos el coeficiente de correlación para ventanas de $2^{\circ} \times 2^{\circ}$ encontramos que el coeficiente de correlación varía entre valores muy cercanos, $-0.8906<$ ce $<-0.6749$ para toda la región.

En la Figura 5.7c se muestra el mapa de la diferencia entre el cálculo de la anomalía isostática sin considerar la presencia de los sedimentos y teniéndolos en cuenta. Aquí también advertimos que dicha diferencia esta correlacionada con la distribución de los espesores de los sedimentos (Fig. 5.3).

Resulta interesante observar en la Figura 5.7b que el máximo de la anomalía isostática en la zona de la cuenca del Colorado (aproximadamente en los $40^{\circ} \mathrm{S}$ de latitud) se manifiesta en la Figura 5.6a como una zona donde el manto es más somero que a sus alrededores, lo cual estaría de acuerdo con que la cuenca se encontraría aún en subsidencia [48]. Esto mismo puede observarse en la Figura 5.8c en donde además se tiene la coincidencia con los máximos de menor extensión areal que se encuentran hacia el oeste. Notar que la Figura 5.6a tiene una extensión en latitud mayor que la Figura 5.8c.

\subsubsection{Deflexión del Moho necesaria para anular la anomalía isostática}

La anomalía isostática refleja el estado de equilibrio en el que se encuentra la corteza, cuyo cambio de espesor representa el mecanismo de compensación de masas en este caso según el modelo de Airy. En la Figura 5.7 se observa que la región se halla parcialmente compensada pero no en la zona del talud. Podemos considerar el ejercicio de atribuir esta no-compensación a la superficie corteza-manto a través de una inversión. Esto lo haremos construyendo una nueva topografía del Moho, aquella que en teoría haría nula la anomalía isostática en superficie. Al ser un proceso de inversión, ya que a partir de datos de gravedad se va a obtener el relieve, el factor exponencial de la transformada de Fourier es positivo y esto hace que se amplifiquen las longitudes de onda corta, por lo que es necesario recortarlas aplicando un filtro pasa bajos a la anomalía isostática. En el proceso de suavizar la anomalía isostática observada, se aplica un filtro con longitud de onda de corte elegida por prueba y error, entre $800 \mathrm{~km}$ y $50 \mathrm{~km}$.

$$
C_{\text {Parker_inv }}=\frac{1}{2 \pi G\left(\rho_{m}-\rho_{c}\right)}
$$

Siendo $G$ la constante de gravitación universal, $\rho_{m}$ la densidad del manto y $\rho_{c}$ la densidad de la corteza. 
(a)

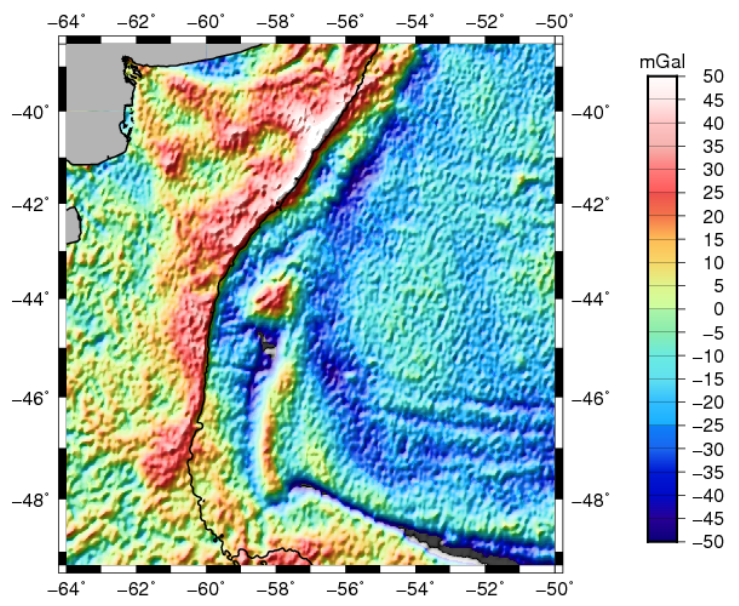

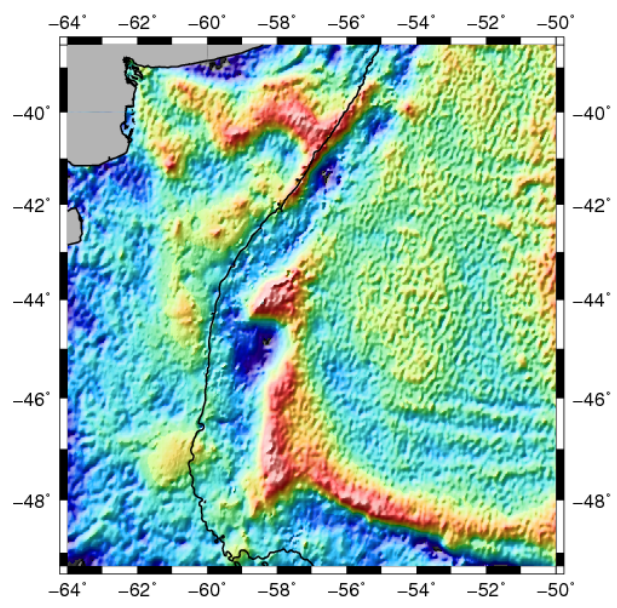

(b)

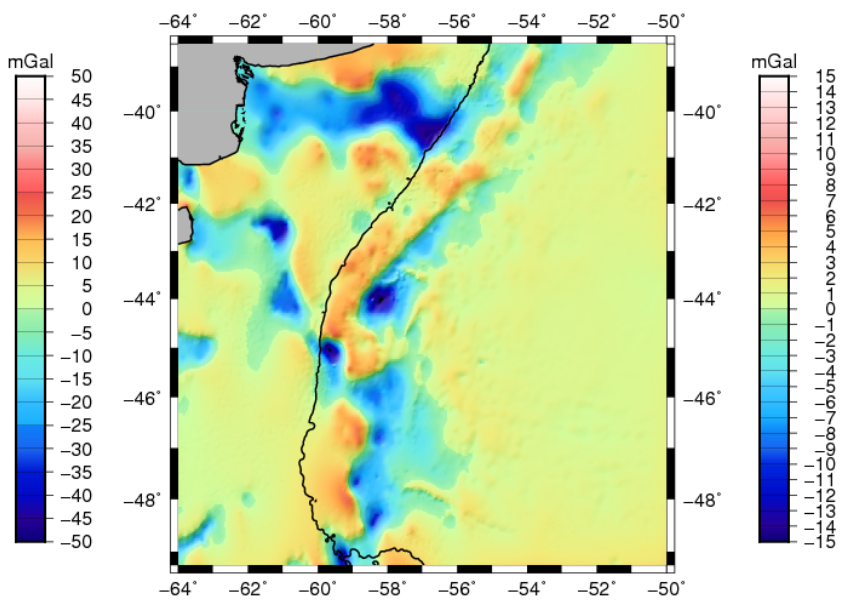

(c)

Figura 5.7. Anomalía de aire libre (a) arriba, anomalía isostática Parker $\mathrm{n}=4$ (b) abajo (izquierda) y diferencia de anomalía isostática Parker $\mathrm{n}=4$ sin sedimentos y con sedimentos (c) abajo (derecha). En las tres figuras se ha dibujado la isobata de $500 \mathrm{~m}$, en color negro, para referencia. 


\section{Isostasia}

Por lo que la ecuación 5.2 puede reescribirse como:

$$
C_{\text {Parker_inv }} \Delta G(k) \exp [2 \pi|k| d]=H(k)
$$

Utilizando la anomalía isostática en la ecuación 5.16 se invierte la topografía $H(k)$ que contiene la deflexión del Moho necesaria para anular dicha anomalía.

En este paso la anomalía isostática residual se atribuye a un relieve en la interfaz corteza- manto, es decir cuánto debería valer la contribución corteza-manto en el cálculo de la anomalía isostática para compensarla totalmente e idealmente obtener una anomalía isostática nula. Lo que intentamos hacer es ver qué forma tendría la interfaz corteza-manto y como sería su relieve para este caso supuesto. Surgen preguntas como: ¿El relieve del manto es muy abrupto?, ¿Es razonable la rugosidad que presenta o es inadmisible para esta interfaz?. Algunas respuestas pueden hallarse al contrastar en regiones con perfiles de refracción disponibles. En nuestro caso sólo tenemos acceso a un perfil digitalizado [30]. El cálculo de la superficie del Moho se obtiene:

1) a partir del conocimiento de la batimetría, el espesor sedimentario y el espesor de la corteza que llamamos Moho 1 (sección 5.2.1 y Fig. 5.6a).

2) utilizando la anomalía isostática hallada con grdfft obteniendo el Moho que llamamos Mohofin1 (Fig. 5.8a), es decir usando un término en la expansión de Parker,

3) utilizando la anomalía isostática hallada con gravfft, requiere más términos, obteniendo el Moho que llamamos Mohofin2 (Fig. 5.8b), notar que en los casos 2) y 3) solo consideramos un término en la inversión, pero en el caso de usar gravfft la anomalía isostática estimada se ha calculado con cuatro términos en la expansión de Parker en el cálculo directo.

4) Una mejor manera de invertir el campo gravimétrico para obtener la topografía de la superficie del Moho se logra utilizando más términos en la inversión como, por ejemplo, utilizando el método iterativo de Parker-Oldenburg [70,67] que ha sido programado en Matlab en la rutina 3Dinver.m por Gómez-Ortiz y Agarwal en 2005 [39]. El programa 3Dinver.m calcula una interfaz de densidad constante con geometría 3D utilizando datos de anomalía de gravedad y el método iterativo de Parker-Oldenburg, basado en el desarrollo de Parker que vincula la transformada de Fourier de la anomalía de gravedad y la suma de las transformadas de la interfaz topográfica (ec. 5.1), conocida su densidad y su profundidad media. El proceso iterativo termina cuando el error cuadrático medio (rms, por sus siglas en inglés) alcanza una cierta tolerancia que sirve como criterio de convergencia, además incorpora un filtro corta altos para mejorar la convergencia del proceso iterativo.

En el paso 4) estamos suponiendo sólo 2 interfaces: agua-corteza y corteza-manto. Hemos utilizado como datos de entrada a 3Dinver.m la anomalía de Bouguer atribuida a la interfaz corteza-manto (Moho), calculada con el programa FA2BOUG [34] para trabajar con un dato independiente de los cálculos que usan el desarrollo de Parker. Como el programa necesita una estimación de la profundidad media de la interfaz a invertir, utilizamos la estimada a partir del Moho1 (Fig. 5.6a).

En la Figura 5.8 se ha representado el Moho calculado inversamente con grdfft (Mohofin1 (a)), con gravfft (Mohofin2 (b)) y con 3Dinver.m (Moho3Dinv (c)). Los dos primeros representan la deflexión del Moho1 para compensar la anomalía isostática, por tanto son el resultado de un experimento. El tercero fue hecho con un método diferente y resulta también en un Moho que compensa casi totalmente la anomalía isostática, 
(a)

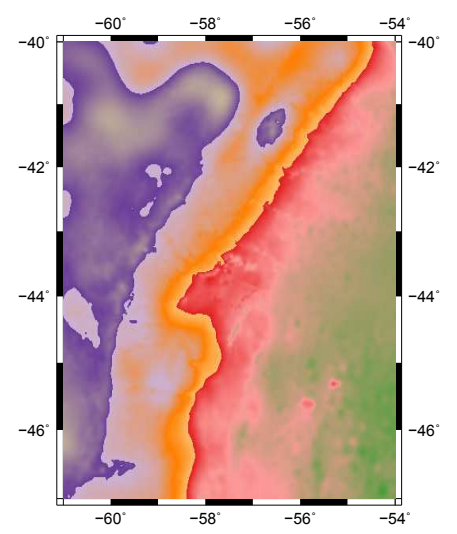

(b)

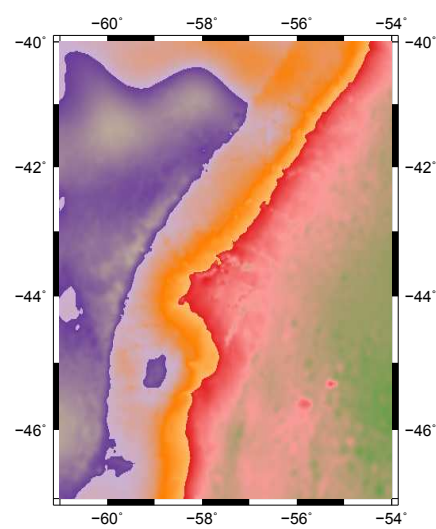

(c)

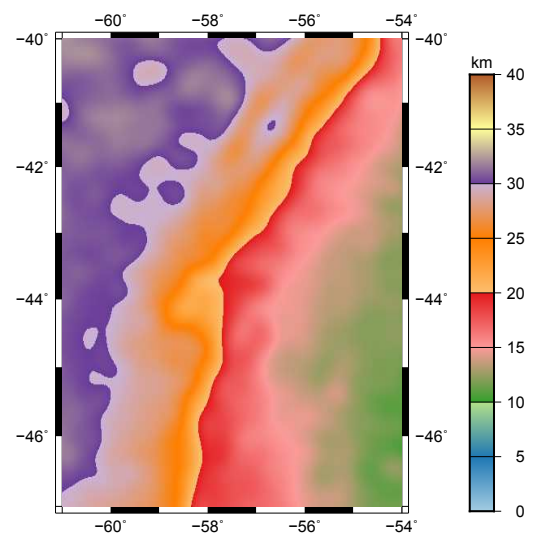

Figura 5.8. Relieve del Moho calculado de tres formas diferentes: que compensa la anomalía isostática (ver texto) (a) Mohofin1 y (b) Mohofin2 y el que se obtiene al invertir la anomalía de Bouguer (c) Moho3Dinv.

hace que la anomalía de aire libre calculada sea muy similar a la anomalía de aire libre observada, como se verá en la siguiente sección. El Moho obtenido a partir de la inversión con el programa 3Dinver.m necesitó 5 iteraciones para un criterio de corte de un rms de 0.02 entre una iteración y la siguiente.

La Figura 5.6a y las Figuras 5.8a, 5.8b y 5.8c representan el resultado de hipótesis isostáticas con base en el modelo de Airy y nos dan una estimación acerca de cómo es la superficie corteza-manto según un modelo de isostasia local de Airy (Moho1, Fig. 5.6a), cómo es si se le atribuye ser la generadora de la anomalía residual (Mohofin1 y Mohofin2, Fig. 5.8a y 5.8b), respectivamente y cómo es para un modelo de dos capas y con un método de inversión más preciso (Moho3Dinv, Fig. 5.8c), pero para conocer cuan buenas son algunas de esas estimaciones necesitamos comparar con un dato más veraz de estas interfaces en profundidad como el que da la refracción sísmica. Para esto se dispone de un perfil de refracción sísmica que ha sido digitalizado del publicado por Franke [30], que veremos en la sección 5.3.6.

En las Figuras 5.8a, 5.8b y 5.8c se observa el carácter morfológico del margen y el cambio abrupto de profundidades del Moho coincidentes con la región del talud. Para visualizar el comportamiento de cada una de estas superficies al atravesar este gradiente de profundidades, se toman perfiles aproximadamente perpendiculares a la dirección del margen (Fig. 5.9), donde puede verse que los perfiles del Moho: Mohofin1 y Mohofin2 presentan una rugosidad local abrupta que los hace parecer inadecuados para la interfaz corteza-manto, como se observa en las Figuras 5.9a, 5.9b, 5.9c y 5.9d.

A partir del Moho invertido con las rutinas grdfft y gravfft (pasos 2) y 3)) pueden recalcularse la anomalía de gravedad de esta interfaz y se espera que la anomalía isostática a posteriori sea cercana a cero (Fig. $5.11 \mathrm{a}, 5.11 \mathrm{~b}, 5.11 \mathrm{c}$ y $5.11 \mathrm{~d}$ ), ya que el experimento consiste en atribuir la anomalía isostática residual a un cambio en la topografía de la interfaz corteza-manto. Asimismo, también se obtiene una anomalía isostática muy cercana a cero para los cálculos derivados del método iterativo. Cabe tener en cuenta que ésta experimentación numérica no pretende hallar la anomalía isostática real sino 
(a)
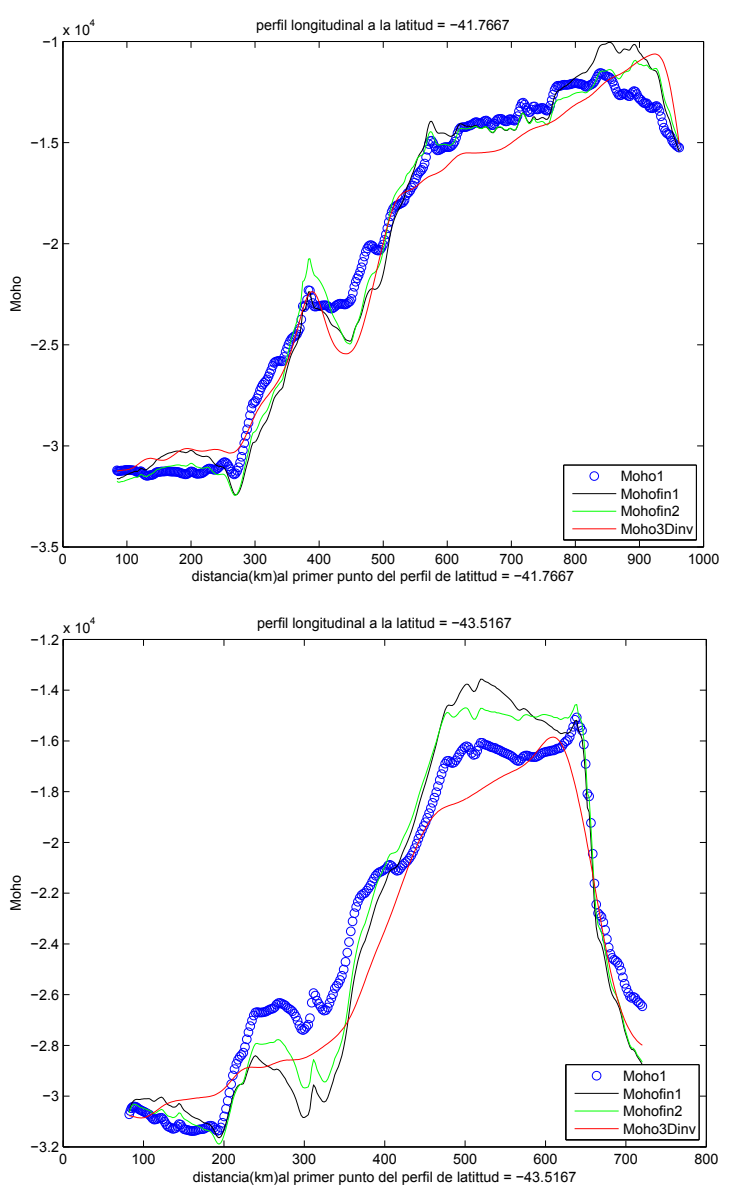

(c) (b)
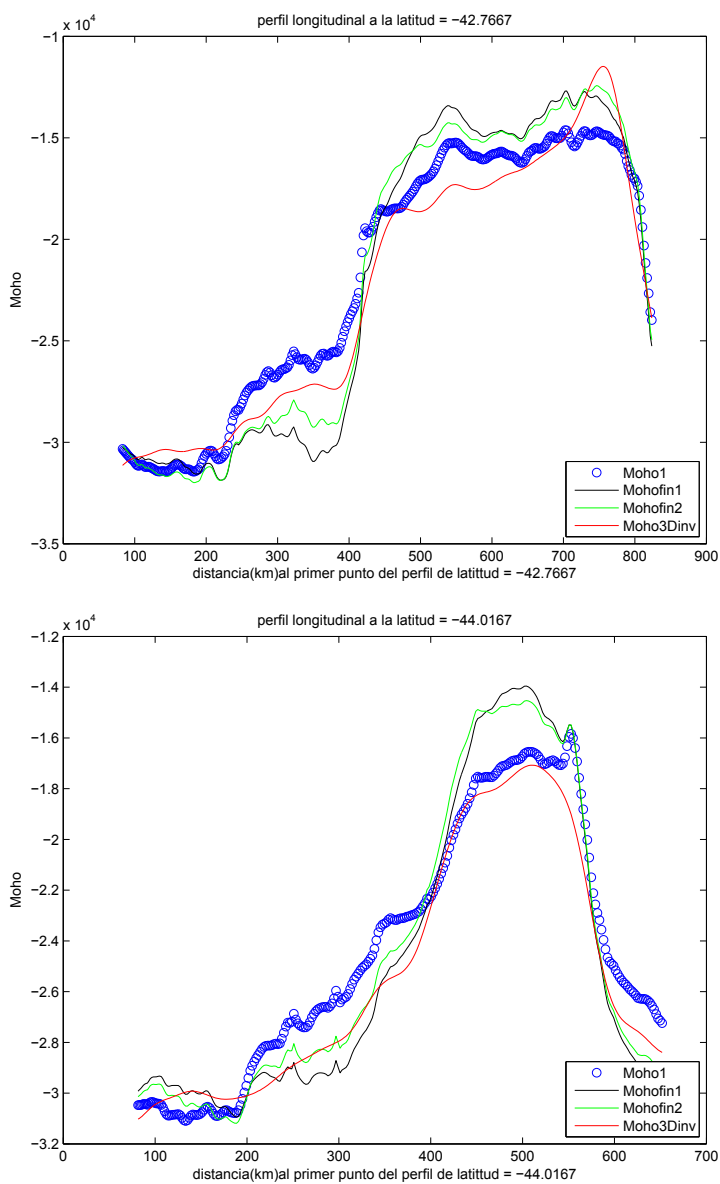

(d)

Figura 5.9. Relieve del Moho que compensa la anomalía isostática, calculado de tres formas diferentes a distintas latitudes (ver texto) -41.77 (arriba, izquierda)(a) -42.77 (arriba, derecha) (b). -43.52 (abajo, izquierda) (c). - 44.02 (abajo, derecha) (d). 
(a)
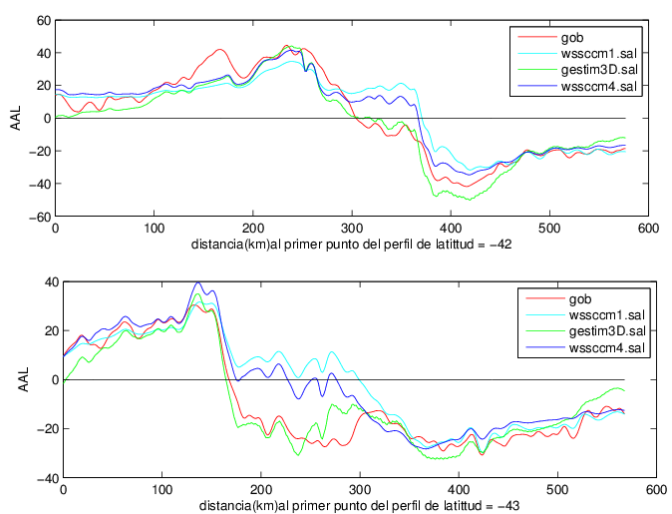

(c) (b)
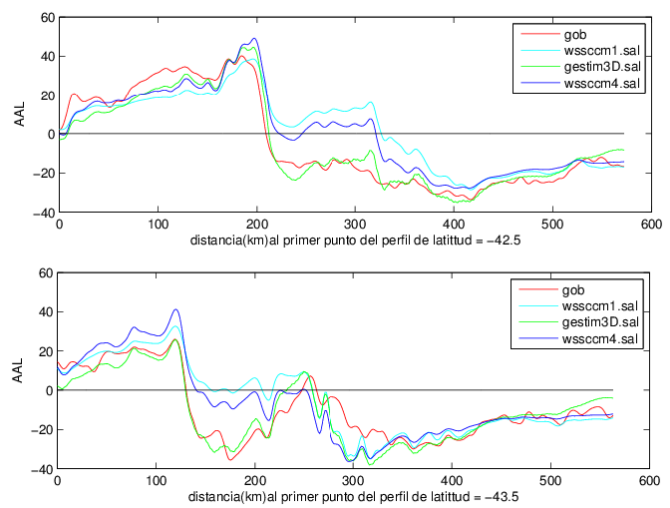

(d)

Figura 5.10. Perfiles a $42^{\circ} \mathrm{S}(\mathrm{a}), 42.5^{\circ} \mathrm{S}$ (b), $43^{\circ} \mathrm{S}$ (c) y $43.5^{\circ} \mathrm{S}$ (d) de latitud, de la anomalía de gravedad de aire libre observada (gob) y estimada con 3 interfaces (wssccm1 (grdfft), wssccm4 (gravfft) y 3Dinv(gestim3D). Notar que wssccm1 y wssccm4 fueron calculadas con el método de tres interfaces usando un Moho obtenido por compensación hidrostática de masas, mientras que gestim3D fue calculada de un Moho derivado de invertir la anomalía de Bouguer. Esto explicaría las diferencias grandes que se observan por ejemplo entre 300 y $400 \mathrm{~km}$ para el perfil a los -42 y entre 180 y $300 \mathrm{~km}$ para el perfil a los -43 . 
(a)

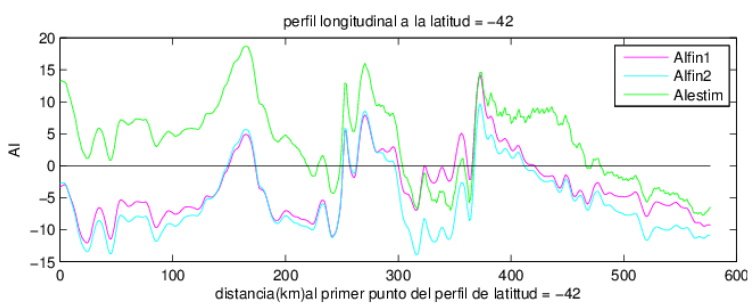

perfil longitudinal a la latitud $=-43$

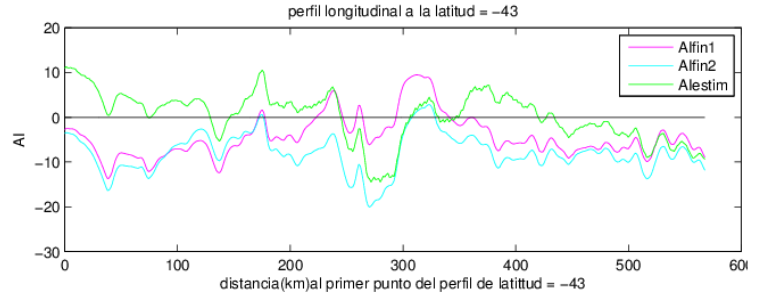

(c) (b)
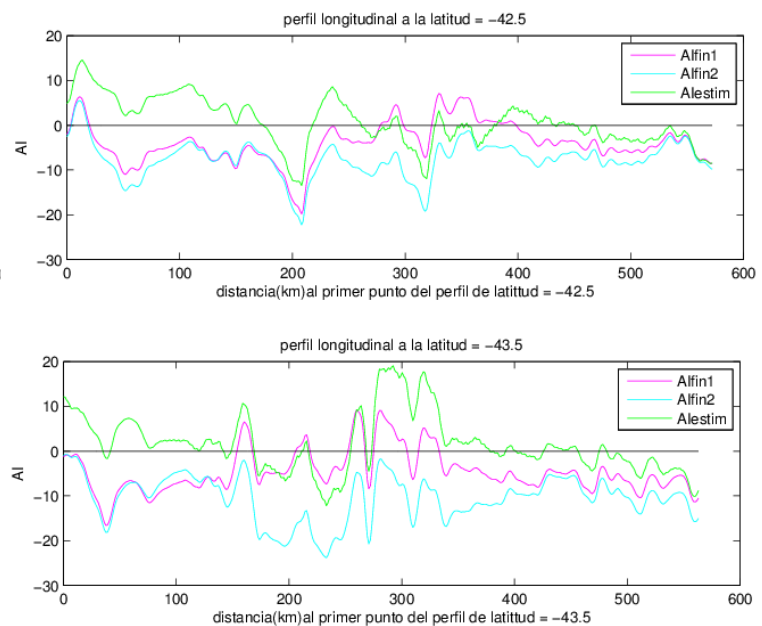

(d)

Figura 5.11. Perfiles a $42^{\circ} \mathrm{S}(\mathrm{a}), 42.5^{\circ} \mathrm{S}$ (b), $43^{\circ} \mathrm{S}$ (c) y $43.5^{\circ} \mathrm{S}$ (d) de latitud, de la anomalía isostática calculada a partir del Mohofin1 (AIfin1), Mohofin2 (AIfin2) y a partir del Moho3Dinv (isoestim).

probar y controlar el funcionamiento de diferentes métodos de cálculo.

\subsubsection{Comparación de anomalías de aire libre}

Para observar las diferencias entre los métodos de inversión graficamos las anomalías de aire libre en perfiles longitudinales (Fig. 5.10) a lo largo del margen. En ellos puede verse cómo la interfaz invertida con el método iterativo (gestim3D), es capaz de representar la anomalía de gravedad calculada acercándosele estrechamente a la observada. La anomalía de aire libre con el método de las tres interfaces se calcula sumando la contribución de los efectos gravimetricos de las tres interfaces (ws+sc+cm). Si el cálculo se realiza con grdfft llamamos wssccm1, donde el 1 representa el hecho de que se calcula para el desarrollo de Parker con $n=1$, si se quiere calcular para otro grado diferente se utiliza gravfft y llamamos a la estimación wssccm 4 , donde el 4 representa $n=4$. Con el programa 3Dinv.m (4) de la sección 5.3.4) puede recalcularse la anomalía debida a la interfaz corteza-manto invertida (anomalía de Bouguer estimada) que puede utilizarse para obtener la anomalía de aire libre estimada (gestim3D en las Fig. 5.10a, 5.10b, 5.10c y 5.10d). El término correspondiente a la contribución agua-corteza (wc) es aquí calculado con gravfft, por lo que gestim3D representa una combinación entre cálculos de 3Dinv.m y de gravfft y la obtenemos despejando de la ecuación 4.3 del Capítulo anterior:

$$
\text { gestim } 3 D \simeq \Delta \tilde{g}_{B}+w_{c}-w_{\text {losa }}
$$

donde $\Delta \tilde{g}_{B}$ representa la anomalía de Bouguer estimada a partir del Moho3Dinv.

En la Figura 5.10 se presentan una serie de perfiles longitudinales donde se muestra la anomalía de aire libre observada (gob), la anomalía de aire libre calculada en forma directa con grdfft (wssccm $1=w s+s c+c m, n=1)$ y con gravfft $(w s s c c m 4=w s+s c+c m$, $n=4$ ), y la anomalía de aire libre obtenida a partir de la interfaz invertida con 3Dinv.m 


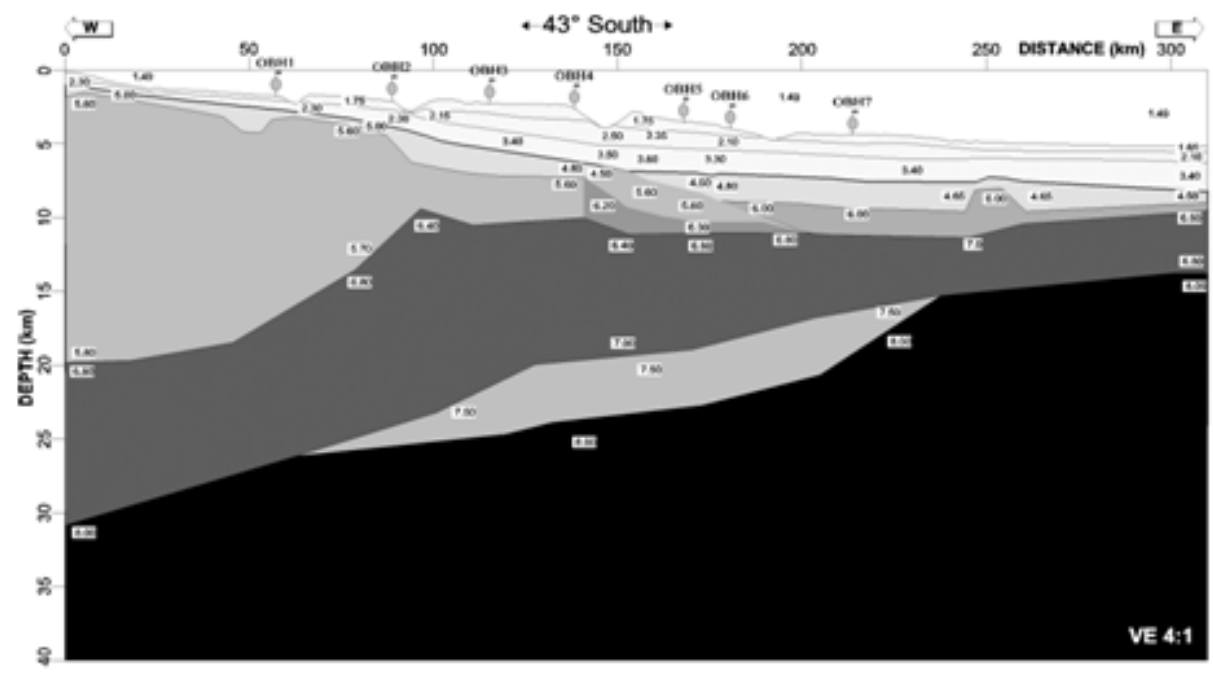

Figura 5.12. Perfil EO de refracción de la campaña BGR98 a la latitud -43.5 [30], interfaces digitalizadas (batyFranke, Mohod-Franke, MohouFranke.

(gestim3D) y la ecuación 4.3 del Capítulo anterior. Puede observarse como la anomalía calculada gestim3D aproxima muy bien a la anomalía observada como era de esperar a modo de comprobación del buen uso del método iterativo al invertir la superficie Moho, mientras que las otras estimaciones son cálculos de la anomalía suma de las tres interfaces para estimar la anomalía de la cual obtenemos la anomalía isostática.

\subsubsection{Comparación con un perfil de refracción}

En la Figura 5.13 se han graficado las interfaces modeladas por Franke y colaboradores (2012) [30] a partir de un perfil de refracción a la latitud $43.5^{\circ} \mathrm{S}$ (Fig. 5.12). Se muestran la batimetría, la profundidad del basamento, y el Moho digitalizado de dicho perfil (baty-Franke), (prof-bas-Franke) y (Moho-Franke), respectivamente; los Mohos Moho1, Mohofin2 y Moho3Dinv, respectivamente; la profundidad del basamento pofbas1 y la batimetria del modelo de Sandwell y Smith batyS\&S

Comparando las interfaces obtenidas con las del perfil de refracción, se puede apreciar que nuestras estimaciones de la interfaz corteza-manto coinciden muy bien con la de dicho perfil, excepto en la zona de la presencia de una capa (AV) de alta velocidad (> $7.0 \mathrm{~km} / \mathrm{s})$, característica de los márgenes volcánicos, ubicada en la corteza media e inferior, debajo de los reflectores sísmicos intra-basamentales que inclinan hacia el mar (SDRS) [66]. Debajo de la capa AV, entre lo que sería el Moho normal (Mohou_Franke, en Fig. 5.13) y el manto, hay un cuerpo $(\mathrm{AV}+)$ de velocidad un poco más alta, 7.5 $\mathrm{km} / \mathrm{s}$ entre Mohou_Franke y Mohod_Franke de aproximadamente 200km de extension lateral y $5 \mathrm{~km}$ de alto en su parte media. Nuestras determinaciones del Moho coinciden con la de la interfaz entre las capas AV + y el manto para casi la totalidad de los perfiles y atraviesan el cuerpo $(\mathrm{AV}+)$ para distancias al primer punto del perfil de entre 450 $\mathrm{km}$ y $550 \mathrm{~km}$. 


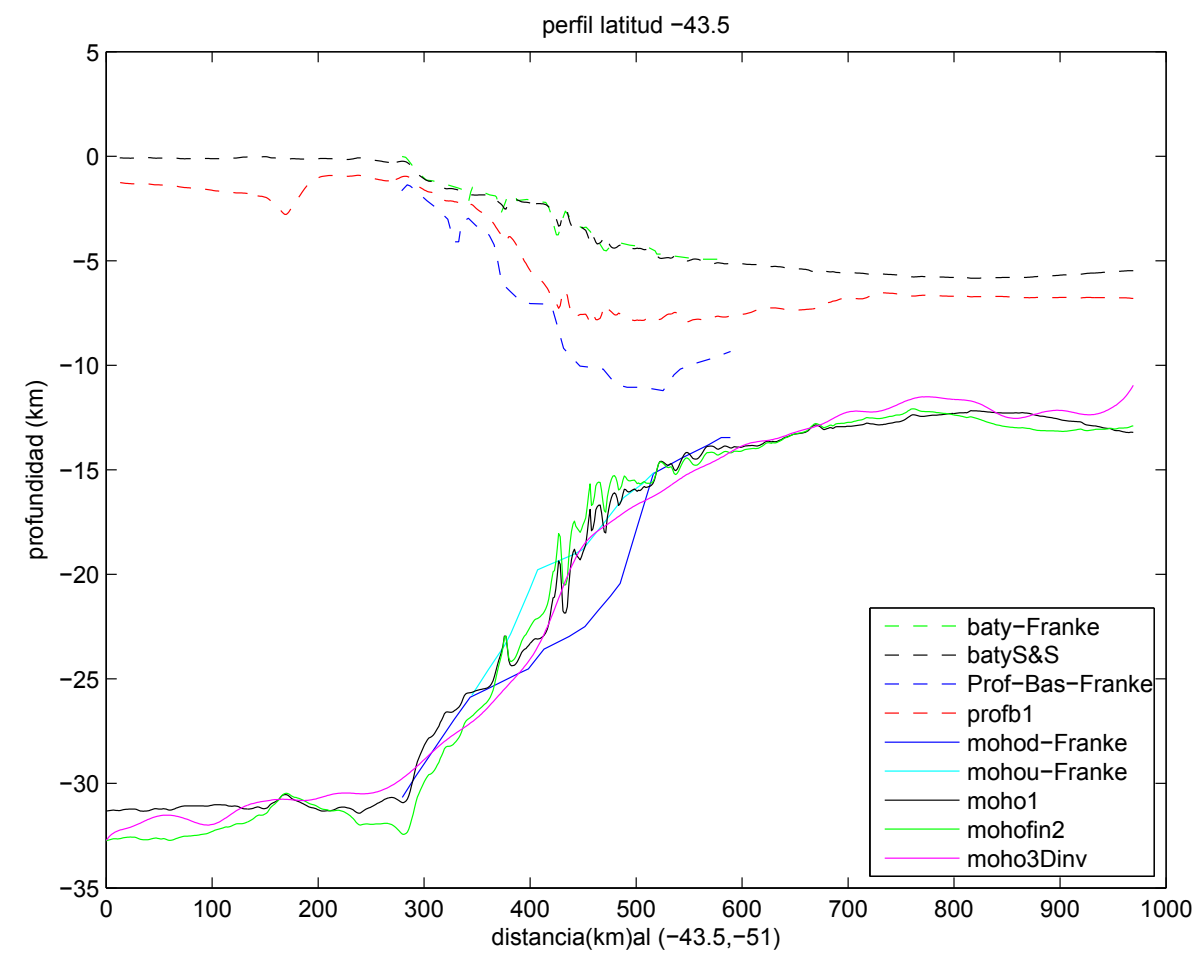

Figura 5.13. Perfiles longitudinales $43^{\circ} \mathrm{S}$ digitalizado de Franke y de las interfaces profb1: profundidad del basamento, batyS\&S: batimetría [79], Moho1 (sección 5.3.4 1)), Mohofin2 (sección 5.3.4 2)), Moho 3D (sección 5.3 .4 3)). Las líneas Mohou-Franke y Mohod-Franke encierran la capa de alta velocidad (AV+,aproximadamente entre los $350 \mathrm{~km}$ y los $550 \mathrm{~km}$ ). 


\subsection{Isostasia Flexural}

Vening Meinesz [91] consideraba a la topografía como una carga en la superficie de la corteza, la que causaría un combamiento a la misma. Si la carga fuese grande, las fuerzas verticales hacia abajo en una corteza rígida dentro de un sustrato dúctil causarían una raíz de baja densidad similar a la predicha por el modelo de Airy, salvo que dicha raíz sería más amplia debido a que la carga esta soportada en parte por la rigidez de la corteza. Walcott fue uno de los primeros en examinar la evidencia de que diferentes cargas en la litosfera producían una flexura, algunas de las cargas que él analizó son lagos glaciares [94], sedimentos [95] e islas oceánicas [93]. El comparó modelos de placas sencillas con la topografía observada y anomalías de gravedad en las regiones de estas cargas, para estimar la rigidez flexural, en un amplio rango de arreglos tectónicos. Su investigación se basó en uno de los pilares de la tectónica de placas: la idea de que las placas se comportan como rígidas a escala de tiempo geológicas. Walcott mostró que el espesor elástico (determinado por la rigidez flexural) de la litosfera en las regiones de grandes cargas, varía entre 5 y $114 \mathrm{~km}$ y estaba de acuerdo en que existía una correlación entre el espesor elástico y la edad: valores grandes de espesor elástico se relacionan con cargas de corta duración (como las de lagos glaciares), mientras que para valores pequeños se asocian con cargas de larga duración (como las islas oceánicas)[103].

\subsubsection{Aplicación de la teoría de flexión de placas a la litósfera de la Tierra}

La deflexión de una placa en dos dimensiones debido a una carga lineal presionando en su superficie, ha sido desarrollada por Turcotte y Schubert (1982) [89] Apéndice A. Cuando se aplica la ecuación general de la deflexión de una placa $D \frac{d^{4} w}{d x^{4}}=q(x)-P \frac{d^{2} w}{d x^{2}}$ (ec. A.14 del Apéndice A, [89]) para determinar la deflexión de la litosfera de debido a una carga aplicada, debemos incluir en $q(x)$ la fuerza de restauración hidrostática causada por el reemplazo de rocas del manto por material de una densidad más baja en una columna vertical.

En el caso de la litosfera oceánica, el agua rellena "el espacio vacante" que dejan las rocas del manto al moverse por la litosfera deflectada, como se ve en Figura 5.14.

La parte superior de la Figura 5.14 muestra una placa listosférica de espesor $l$ y densidad $\rho_{l}$ flotando en el manto "fluido". El agua de densidad $\rho_{w}$ de espesor $h_{w}$ yace sobre la litosfera oceánica. Supongamos que una carga aplicada deflecta la litosfera hacia abajo una distancia $w$ y el agua rellena el espacio sobre la placa, como puede verse en la parte inferior de la Figura 5.14. El peso por unidad de área de la columna vertical que se extiende desde la base de la litosfera a la superficie es:

$$
\rho_{w} g\left(h_{w}+w\right)+\rho_{l} g l .
$$

La presión a la profundidad $h_{w}+l+w$ en los alrededores del manto donde la placa no está deflectada es:

$$
\rho_{w} g h_{w}+\rho_{l} g l+\rho_{m} g w .
$$

Hay una fuerza hidrostática por unidad de área, hacia arriba:

$$
\rho_{w} g h_{w}+\rho_{l} g l+\rho_{m} g w-\left\{\rho_{w} g\left(h_{w}+w\right)+\rho_{l} g l\right\}=\left(\rho_{m}-\rho_{w}\right) g w
$$



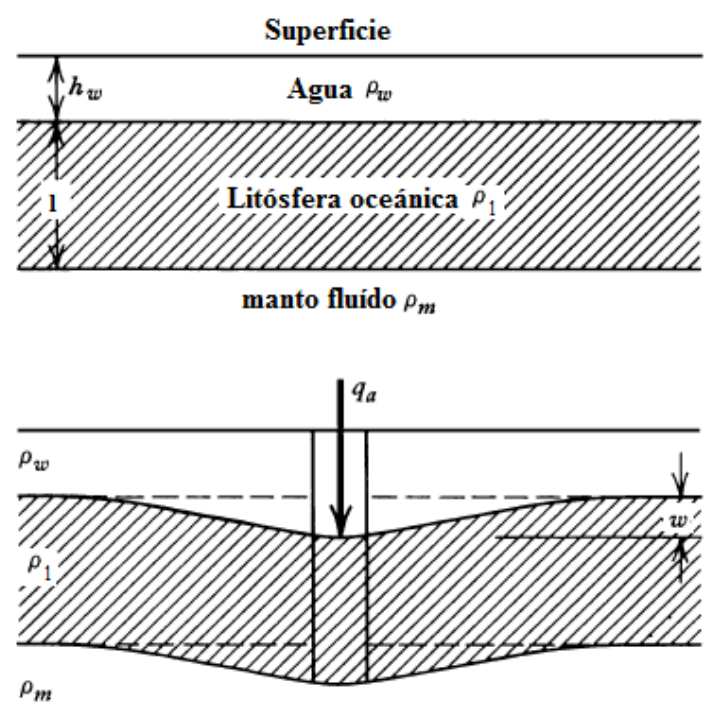

Figura 5.14. Modelo para el cálculo de la fuerza restitutiva en las placas litosféricas deflectadas por una carga aplicada $q_{a}$ para el caso oceánico (Turcotte \& Schubert) [89].

la que tiende a restablecer la deflexión de la litosfera a la configuración original. Donde $\rho_{m}$ es la densidad del material debajo de la placa y $\rho_{w}$ es la densidad del material encima de la placa.

La fuerza restitutiva por unidad de área es equivalente a la fuerza que resulta de reemplazar las rocas del manto de espesor $w$ y densidad $\rho_{m}$. La fuerza neta por unidad de área actuante en la placa litosférica es:

$$
q=q_{a}-\left(\rho_{m}-\rho_{w}\right) g w
$$

donde $q_{a}$ es la carga aplicada en la parte superior de la litósfera. La ec. A.14 para la deflexión de la litósfera oceánica queda:

$$
D \frac{d^{4} w}{d x^{4}}+P \frac{d^{2} w}{d x^{2}}+\left(\rho_{m}-\rho_{w}\right) g w=q_{a}(x)[89] .
$$

Para el caso de una placa litosférica deformada bajo una carga sedimentaria se tiene:

$$
q_{a}(x)=\left(\rho_{s}-\rho_{w}\right) g h(x)[20]
$$

donde $\mathrm{h}(\mathrm{x})$ es la altura de carga.

\subsubsection{Diagnóstico del ancho de banda de la flexura}

La litosfera responde a las cargas geológicas a largo plazo no localmente como predicen los modelos de Airy y Pratt, sino regionalmente por flexura en una amplia región. En ese sentido la litosfera se comporta como un filtro que suprime la deformación de corta longitud de onda asociada con los modelos de isostasia local y deja pasar la deformación de larga longitud de onda asociada con la flexura. Tal filtro se caracteriza 
por una entrada que es la carga y la salida que es la flexura, es lineal e invariante, es decir si una carga $h_{1}(x)$ produce una deflexión $w_{1}(x)$ y otra carga $h_{2}(x)$ produce una deflexión $w_{2}(x)$ entonces la carga $h_{1}(x)+h_{2}(x)$ produce una deflexión $w_{1}(x)+w_{2}(x)$. Una propiedad de filtros de este tipo es que cuando se somete a una carga periódica, la salida también es periódica [103]. Considerando el caso oceánico de una placa elástica que yace sobre un sustrato dúctil suponiendo que no hay esfuerzos laterales $(P=0)$ y sometida a una carga periódica:

$$
D \frac{\partial w^{4}}{\partial x^{4}}+\left(\rho_{m}-\rho_{\text {relleno }}\right) w g=\left(\rho_{c}-\rho_{w}\right) g h \cos (k x)
$$

donde $D$ es la rigidez flexural, $w$ es la flexura, $x$ es la distancia horizontal, $\rho_{m}$ y $\rho_{\text {relleno }}$ son las densidades del manto y del material que rellena la depresión de la flexura, respectivamente, $h$ el pico de la amplitud de la carga y $k$ el número de onda de la carga en la dirección de $\mathrm{x}\left(k=2 \pi / \lambda_{w} ; \lambda_{w}\right.$ es la longitud de onda $), \rho_{c} \mathrm{y} \rho_{w}$ son las densidades de la carga y del medio que desplaza la carga (agua o aire respectivamente).

La solución de ecuación 5.23 es periódica y dada por:

$$
w=\frac{\left(\rho_{c}-\rho_{w}\right) h \cos (k x)}{\left(\rho_{m}-\rho_{\text {relleno }}\right)}\left[\frac{D k^{4}}{\left(\rho_{m}-\rho_{\text {relleno }}\right) g}+1\right]^{-1}
$$

donde si se hace tender la rigidez a cero $(D \rightarrow 0)$, lo que se correspondería con una placa débil, la solución queda:

$$
w \rightarrow \frac{\left(\rho_{c}-\rho_{w}\right) h \cos (k x)}{\left(\rho_{m}-\rho_{\text {relleno }}\right)}
$$

para la cual se obtiene la respuesta isostática de Airy a la carga periódica si $\rho_{\text {relleno }} \rightarrow \rho_{c}$, además podemos observar que si $D \rightarrow \infty$ (la placa es totalmente rígida) por lo que:

$$
w \rightarrow 0
$$

que es la respuesta de Bouguer.

En síntesis si la placa es débil, su respuesta a las cargas es local y aproximadamente la del modelo de Airy. Si la placa es rígida, no hay respuesta, que es el caso de Bouguer. Cuando la placa tiene alguna rigidez intermedia finita, su respuesta está dada por la flexura.

Si consideramos la entrada y salida de un sistema, podríamos escribir el parámetro $\Phi_{e}(k)$, que modifica la respuesta de Airy (Entrada) a la que produce la flexura (Salida) como:

$$
\begin{array}{r}
\Phi_{e}=\frac{\text { Salida }}{\text { Entrada }} \\
\text { Entrada }=\frac{\left(\rho_{c}-\rho_{w}\right) \text { hcos }(k x)}{\left(\rho_{m}-\rho_{\text {relleno }}\right)} \\
\text { Salida }=\frac{\left(\rho_{c}-\rho_{w}\right) h \cos (k x)}{\left(\rho_{m}-\rho_{\text {relleno }}\right)}\left[\frac{d k^{4}}{\left(\rho_{m}-\rho_{\text {relleno }}\right) g}+1\right]^{-1} \\
\therefore \Phi_{e}=\left[\frac{D k^{4}}{\left(\rho_{m}-\rho_{\text {relleno }}\right) g}+1\right]^{-1}
\end{array}
$$




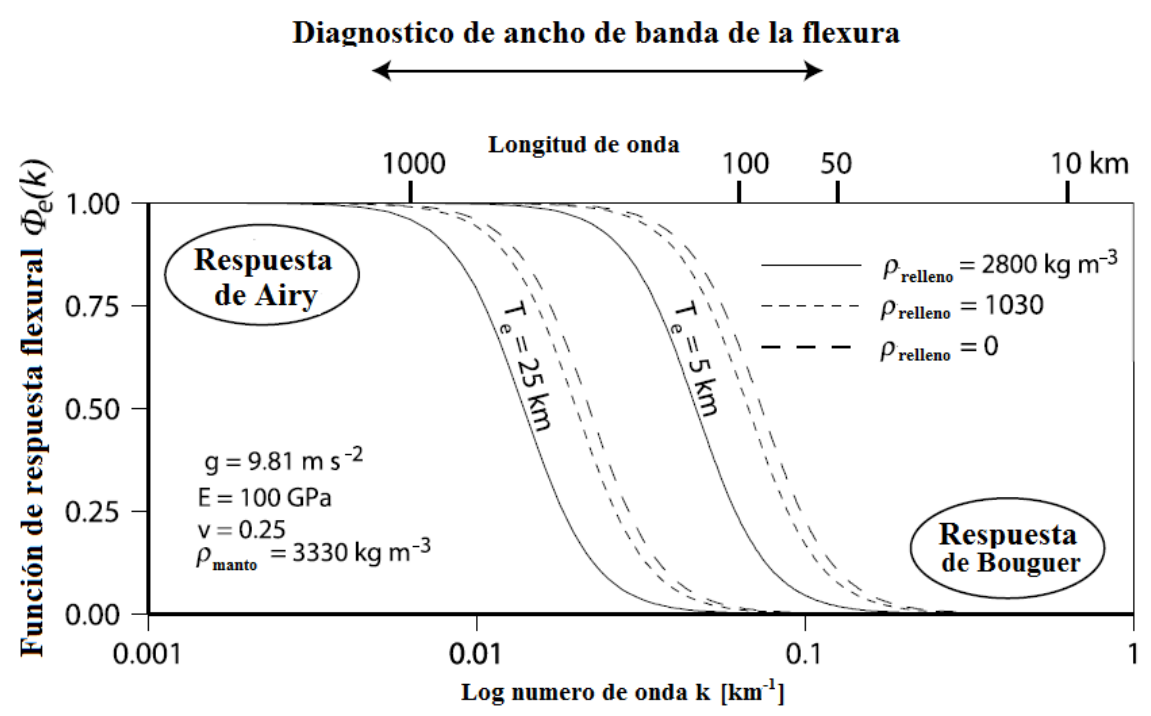

Figura 5.15. Función de respuesta flexural $\phi(k)$, modificado de Watts 2001 [103].

En la Figura 5.15 puede observarse la dependencia de $\Phi_{e}$ con $k$ para un rango de densidades de relleno. Se muestra un set de curvas: para una placa débil $\left(T_{e}=5 \mathrm{~km}\right)$ y otra para una más rígida $\left(T_{e}=25 \mathrm{~km}\right)$. Podemos observar que para números de onda pequeños, longitudes de onda de carga largas, la placa se comporta como débil dando una respuesta de tipo Airy. En contraste para grandes números de onda, cortas longitudes de onda de carga, la placa se comporta como rígida. Este comportamiento se verifica al considerar los valores límites de $k$. Si $k \rightarrow 0$ :

$$
w \rightarrow \frac{\left(\rho_{c}-\rho_{w}\right) h}{\left(\rho_{m}-\rho_{\text {relleno }}\right)}
$$

que es la respuesta de Airy si $\rho_{\text {relleno }} \rightarrow \rho_{c}$. Similarmente, si $k \rightarrow \infty, w \rightarrow 0$ que es la respuesta de Bouguer. Para números de onda intermedios $(0.001<k<0.1)$ la respuesta es la flexural. Watts [103] llamó a este ancho de banda diagnóstico de ancho de banda de la flexura.

La litosfera se comporta como un filtro pasabajos entre la carga y la deformación flexural asociada, siendo el corte en frecuencias, determinado por la rigidez de la placa (y por ende por su $T_{e}$ ).

La ecuación 5.24 da la respuesta flexural para una carga de una particular longitud de onda. Si podemos separar a la carga en de sus componentes espectrales individuales, entonces la ecuación puede usarse para calcular la respuesta de cualquier carga en dos dimensiones de forma arbitraria. Sea la ecuación 5.23 para el caso de una función de carga arbitraria $\mathrm{h}(\mathrm{x})$ y $\rho_{l}$ es la densidad de carga:

$$
D \frac{d^{4} w}{d x^{4}}+\left(\rho_{m}-\rho_{\text {relleno }}\right) g w=\left(\rho_{l}-\rho_{w}\right) g h(x) .
$$

La solución de ésta ecuación es más eficientemente calculada en el dominio de la frecuencia por lo que aplicamos transformada de Fourier en ambos miembros:

$$
D k^{4} \mathbf{W}(k)+\left(\rho_{m}-\rho_{\text {relleno }}\right) \mathbf{W}(k) g=\left(\rho_{l}-\rho_{w}\right) \mathbf{H}(k) g
$$


por lo que puede despejarse la deflexión como:

$$
\mathbf{W}(k)=\Phi_{e}(k) \frac{\left(\rho_{l}-\rho_{w}\right)}{\left(\rho_{m}-\rho_{\text {relleno }}\right)} \mathbf{H}(k)
$$

donde las variables en mayúscula y negrita representan las transformada de las minúsculas y $k=\frac{2 \pi}{\lambda}$ es el numero de onda en la dirección de x y $\lambda$ es la longitud de onda.

La variación espacial de la flexura debido a una carga en dos dimensiones de forma arbitraria se obtiene primero tomando la transformada de Fourier de la carga, multiplicando por un parámetro y un término de densidad y luego aplicando la transformada inversa.

\subsection{Conclusiones del Capítulo 5}

1) En la primera parte de éste Capítulo se investigó el mecanismo de compensación de tipo Airy con un esquema de tres interfaces utilizando las metodologías que se describen a continuación :

- en sentido directo con diferentes aproximaciones del desarrollo de Parker (utilizando los programas grdfft [106] y gravfft [57]), que permiten obtener de manera simple una anomalía de aire libre, que extraída de la anomalía de aire libre observada da una primera estimación de la anomalía isostática que depende fuertemente de la contribución de los sedimentos.

- en sentido inverso, determinando la deflexión del Moho necesaria para compensar la anomalía isostática calculada en el sentido directo, experimento que no deja de ser una suposición simple para probar los métodos numéricos.

2) la interfaz corteza-manto (Moho) calculada con el programa 3Dinver.m [39] representa una superficie físicamente más aceptable de acuerdo a la rugosidad que presenta. En la comparación con el único perfil de refracción de que disponemos para hacer la comprobación de este cálculo, la interfaz "Moho" invertida con el método iterativo (Moho3D) presenta un buen ajuste con el Moho modelado en dicho perfil.

3) Los programas grdfft y gravfft son mucho más simples y rápidos para la incorporación de carga sedimentaria en el tratamiento de zonas oceánicas amplias (recordar que con 3Dinv se usa la anomalía de Bouguer y dos interfaces: agua-corteza y corteza-manto). 4) Existe una marcada correlación entre los máximos de la diferencia de anomalía isostática, no teniendo en cuenta el efecto de la contribución de los sedimentos y sí teniéndolos en cuenta, y el espesor sedimentario. Se considera crítico contar con una mejor estimación del dato de espesor sedimentario necesario para la inclusión de la contribución de este efecto para una mejor interpretación de la anomalía isostática del margen continental pasivo.

5) En la segunda parte del Capítulo se estudiaron conceptos de isostasia flexural. Si consideramos a la litosfera con una cierta rigidez constante y que bajo las aproximaciones de placa delgada responde a las cargas con un comportamiento flexural, la litosfera se comporta como un filtro pasabajos entre la carga y la deformación flexural asociada, siendo el corte en frecuencias determinados por la rigidez de la placa (y por ende por 
su $\left.T_{e}\right)$.

El método flexural se implementará en el próximo Capítulo para la aplicación de las técnicas del Modelado de Gravedad Orientado por los Procesos (POGM). 


\section{Capítulo 6}

\section{Backstripping y Modelado de Gravedad Orientado por los Procesos}

En éste Capítulo tratamos el Modelado de Gravedad Orientado por los Procesos [102] (POGM, por sus siglas en inglés) que combina la técnica backstripping [86, 100] con técnicas de modelado de gravedad. El backtripping es una técnica cuantitativa basada en la remoción de la carga desde el basamento, que se aplica bajo las hipótesis de isostasia de Airy o flexural. Asume que se conoce la carga sobre la corteza producida por el agua y los sedimentos. A partir de la ecuación del backstripping [103] es posible determinar la subsidencia tectónica directamente desde datos estratigráficos. Con la técnica backstripping podemos estimar la profundidad del basamento en ausencia de sedimentos, es decir la paleobatimetría en el tiempo del rift, la cual hemos calculado teniendo en cuenta todo el espesor de sedimentos sin diferenciación de edades. Para el caso de backstripping flexural se determina un parámetro apropiado en el espacio de los números de onda, que modifica la respuesta de Airy produciendo la respuesta flexural en el dominio de Fourier. El POGM utiliza información sísmica del espesor sedimentario y del volcanismo presente (SDRs, subplacado, etc.) en la configuración del margen actual, para estimar la anomalía de gravedad que está asociada a los procesos: rifting, sedimentación y magmatismo. El objetivo de POGM es discriminar la contribución de los diferentes procesos geológicos a la anomalía observada y el del backstripping es determinar la geometría del margen al tiempo del rift.

\subsection{Cuencas de tipo rift}

Muchas cuencas sedimentarias se generan por estructuras de tipo rift como half-graben, fallas directas y de la corteza continental como lo sugieren perfiles de sísmica de refracción y reflexión. Es por ello que los rifts son importantes precursores en el desarrollo de cuencas[103]. Una cuenca oceánica es quizás el tipo más obvio de cuenca, que ha sido generada por un rift. Estas pueden describirse como depresiones ocupadas por agua que se forman por contracción termal de la corteza oceánica, cuando ésta se enfría, incrementando su densidad y hundiéndose al alejarse de la cresta de la dorsal. El modelo de enfriamiento de placas provee una buena descripción de esta subsidencia. Los 
sedimentos que se acumulan son escasos comparados con la gran acumulación que se encuentra ahora por debajo de aguas profundas en márgenes continentales.

La flexura es un contribuyente importante de la "arquitectura" de continentes y rift oceánicos lo que da un factor de control en el desarrollo de estratigrafía post-rift de márgenes continentales. El problema que se presenta es que muchos de los sedimentos que se acumulan en el margen se depositaron en aguas relativamente someras, esto significa que la carga de los sedimentos en las depresiones ocupadas por agua no puede ser sólo la causa de la subsidencia y por ello deben investigarse otros factores (ver Cap. 2) [103].

Argumentos isostáticos sugieren que independientemente de la causa de la subsidencia, la carga de sedimentos y de agua serían los procesos que más contribuyen al desarrollo de la cuenca de rift. El rol que juega la carga es más pequeño para los sedimentos no consolidados que para los consolidados. Sin embargo, si se desarrolla una técnica para corregir los registros de estratigrafía de los efectos de la sedimentación y la carga de agua se tendrá una herramienta para entender mejor cómo ha sido la subsidencia y por lo tanto el origen de la cuenca de tipo rift.

\subsubsection{Backstripping}

Sleep (1971) [83] realizó uno de los primeros intentos de analizar la historia de la subsidencia de cuencas de rift al graficar la subsidencia en un número de pozos en New Jersey normalizados con respecto a la profundidad de un simple horizonte estratigráfico. Aunque la mayoría de los perfiles analizados por Sleep se localizaban en la depresión flexural en los flancos de la principal carga sedimentaria, él demostró que la subsidencia de cuencas de tipo rift se produce en forma exponencial como en una dorsal centro oceánica. Esta similitud lo llevó a sugerir que los márgenes pasivos se originan por calentamiento de la litosfera en el tiempo del rift [103].

Para normalizar la subsidencia de una cuenca de rift con respecto a un horizonte estratigráfico particular, Sleep (1971) [83] fue capaz de seguir el proceso de acumulación de sedimentos en una cuenca en el tiempo geológico. La acumulación de sedimentos no sólo es consecuencia de procesos tectónicos que son responsables de la formación de la cuenca, sino de los efectos de la carga del agua y de los sedimentos.

Watts y Ryan (1976) [96] desarrollaron una técnica para "corregir" el registro estratigráfico por los efectos del agua y la carga de sedimentos y aislar de esta manera las fuerzas desviatorias tectónicas que fueron responsables de la subsidencia de la cuenca de rift. Para restaurar el espesor sedimentario al tiempo de la depositación, tuvieron en cuenta la compactación y los cambios en el nivel del agua, y la compensación isostática antes de la carga, este trabajo ayudó a determinar la profundidad que tendría el basamento en ausencia de agua y carga de sedimentos. Ellos se refieren a esta técnica como backstripping.

La técnica backstripping [96, 85, 103], que se basa en remover la carga desde el basamento, presupone que conocemos como es la carga ejercida sobre la corteza por el agua y los sedimentos, lo que por supuesto no se conoce. Fuera de esta limitación, la técnica ha probado ser un método útil para el análisis de datos estratigráficos. El objetivo del backstripping es aislar la parte de la subsidencia del margen causada por factores 

a) Observado
b) Decompactado
c) Backsstripping

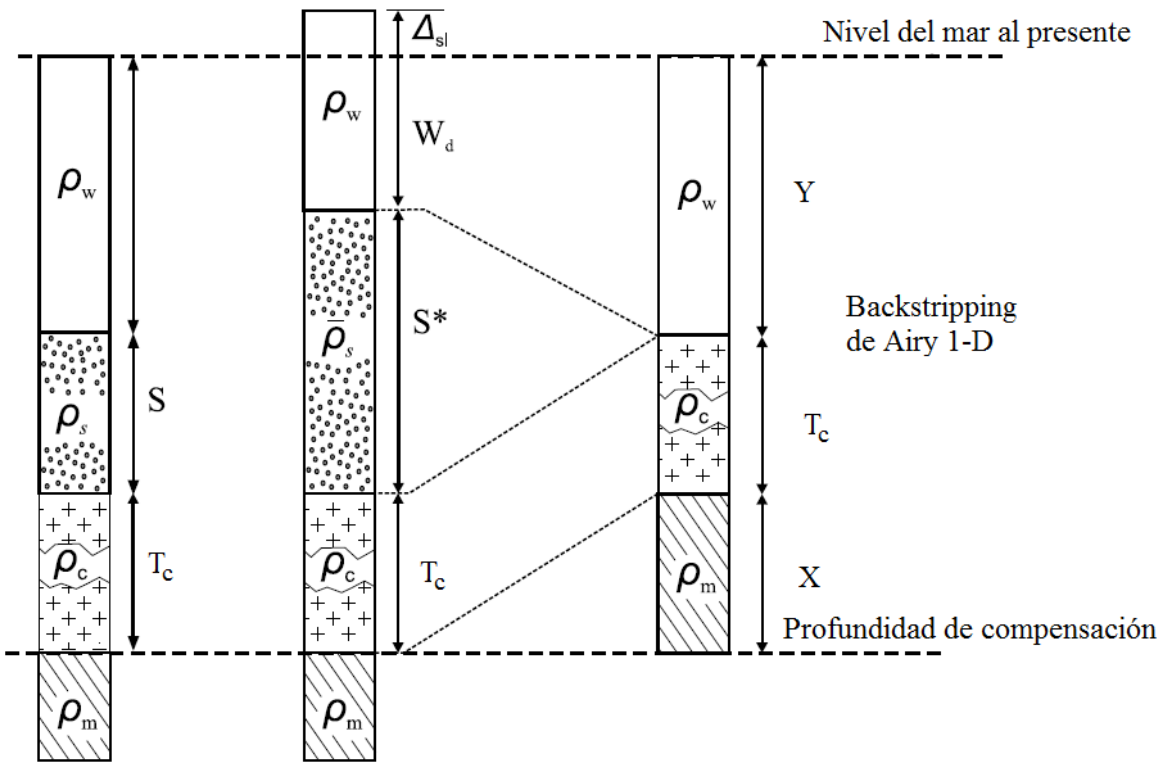

Figura 6.1. Los principios del backstripping tipo Airy, modificado de Cuna [20].

sedimentarios de otros factores tectónicos [97].

El backstripping puede calcularse en una localización puntual (1D) siendo utilizado para derivar curvas de subsidencia tectónica en pozos, donde se tiene información de la estratigrafía y del paleoambiente durante la historia del margen. El backstripping 1D asume que la carga de los sedimentos está localmente compensada, es decir según Airy. Los datos de pozos generalmente pertenecen a compañías petroleras y están fuera del alcance de esta tesis.

El backstripping de sedimentos puede calcularse a lo largo de perfiles (2D) o para un área si se utilizan grillas (3D), en cuyo caso se utiliza un modelo de isostasia regional (flexural) más que local. En general, solo se tiene información detallada de estratigrafía y paleoambientes en el caso 1D, por lo que de ser posible es deseable integrar varias de éstas técnicas [20].

El primer paso en el backstripping 1D es descompactar la columna de sedimentos y reconstruir su espesor y densidad original, al tiempo de la depositación (Fig. 6.1a y 6.1b). Para ello se utilizan curvas empíricas que se derivan de relaciones que tienen en cuenta los efectos de la compactación mecánica con el soterramiento en profundidad de los sedimentos.

Para tener en cuenta el efecto de la compactación en el espesor de sedimentos y la densidad, se asume un modelo simple en el que la compactación es un proceso mecánico irreversible que involucra la expulsión del agua desde los poros de los sedimentos. En otras palabras, los efectos de una alteración química o diagénesis de los sedimentos no se tienen en cuenta. Podemos escribir [103]:

$$
\begin{gathered}
S^{*}=\frac{S\left(1-\phi_{s}\right)}{\left(1-\phi_{s}^{*}\right)} \\
\bar{\rho}_{s}=\rho_{w} \phi_{s}^{*}+\rho_{g}\left(1-\phi_{s}^{*}\right)
\end{gathered}
$$


donde $S$ es el espesor de la capa compactada (el espesor al presente), $S^{*}$ es el espesor de la capa descompactada, $\phi_{s}$ es la porosidad de la capa compactada, $\phi_{s}^{*}$ es la porosidad de la capa descompactada, $\rho_{g}$ es la densidad de los granos de los sedimentos y $\bar{\rho}_{s}$ es la densidad promedio descompactada de una capa sedimentaria particular.

Las ecuaciones 6.1 y 6.2 muestran que el cálculo del espesor y densidad en el tiempo de la depositación requieren una hipótesis acerca de la porosidad de la capa, previo a la compactación. Una aproximación es construir una curva de densidad en profundidad desde curvas "empíricas" basadas en un simple perfil sónico de un pozo. La porosidad de cualquier capa descompactada puede estimarse por "corrimiento" del espesor al presente en la curva de porosidad-densidad. En forma alternativa funciones de porosidad-densidad se asumen basadas en compilaciones de datos de litologías individuales de toda la cuenca [103].

Recuperar la profundidad del basamento en ausencia de la carga de los sedimentos y el agua es un problema de balance de presiones en la base de las columnas descompactada y a la que se le ha aplicado el backstripping (Fig. 6.1b y 6.1c) por lo que:

$$
\rho_{w} g W_{d}+\bar{\rho}_{s} g S^{*}+\rho_{c} g T_{c}=Y \rho_{w} g+\rho_{c} g T_{c}+X \rho_{m} g
$$

donde $W_{d}$, e $Y$ son la profundidad del agua al tiempo de la depositación y la subsidencia tectónica, respectivamente. $T_{c}$ y $\rho_{c}$ son el espesor medio de corteza y la densidad de la corteza, respectivamente, (ambos asumidos como constantes durante la carga y descarga), y $\rho_{w}, \rho_{m}$ son las densidades del agua y manto, respectivamente.

Desde la Fig. 6.1 puede despejarse la altura del manto elevado por encima de la profundidad de compensación:

$$
X=W_{d}+S^{*}+T_{c}-\left(Y+\Delta_{s l}+T_{c}\right)
$$

donde $\Delta_{s l}$ es la altura del valor medio sobre o bajo la superficie de referencia. Haciendo la sustitución y resolviendo [103] para Y, la ecuación 6.3 se convierte en:

$$
Y=W_{d}+S^{*}\left[\frac{\rho_{m}-\bar{\rho}_{s}}{\rho_{m}-\rho_{w}}\right]-\Delta_{s l} \frac{\rho_{m}}{\left(\rho_{m}-\rho_{w}\right)}
$$

La ecuación 6.5 es conocida como "ecuación de backstripping", a partir de la cual puede determinarse la subsidencia tectónica $Y$ [85] directamente desde datos estratigráficos. El primer término en la ecuación es la profundidad del agua, el segundo término está asociado a la carga de los sedimentos, y el tercer término está asociado a la carga del nivel del mar [103].

El término de la profundidad del agua se refiere a la profundidad de la depositación de una unidad estratigráfica particular. Para una unidad depositada en el presente, esto es simplemente la profundidad del agua. Por suerte hay ciertos "indicadores de profundidad" en los que se incluyen fósiles y material geoquímico.

El término de la "carga de sedimentos", describe la manera en que la carga de sedimentos deforma la corteza y el manto. Esto incluye un término de espesor y uno de densidad [103], sin embargo no son cantidades bien determinadas en la actualidad. Esto se debe a que los sedimentos son modificados por procesos como la compactación, la diagénesis, sobrepresión, y otros.

En la práctica, el backstripping se calcula para un tiempo durante la evolución de la 
cuenca y esto involucra remover más de una unidad estratigráfica. El punto importante es que se requiere el espesor total, $S^{*}$, y la densidad promedio, $\bar{\rho}_{s}$, de la secuencia a restaurar en algún tiempo particular. Una cuenca sedimentaria representa la acumulación de más de una unidad, por lo que es necesario hacer el backstripping de todas las unidades para recuperar la subsidencia tectónica (Y) a diferentes tiempos durante la evolución de la cuenca. Para ello el espesor y la porosidad de todas las capas deben recalcularse en cada paso, decompactando las unidades más jóvenes y compactando las más viejas (ver Fig. 6.2).

Para un tiempo particular (Tiempo 1, Tiempo 2 en la Figura 6.2) la densidad promedio de la secuencia se obtiene sumando la masa de todas las unidades restauradas y divididas por el espesor total [85]:

$$
\bar{\rho}_{s}=\frac{\sum_{i=1}^{n}\left[\rho_{w} \phi_{s i}^{*}+\rho_{g i}\left(1-\phi_{s i}^{*}\right)\right] S_{i}}{S^{*}}
$$

donde i es la iésima capa y $\mathrm{n}$ el número total de capas estratigráficas en un tiempo particular, $\bar{\rho}_{s}$ puede substituirse en la ecuación 6.5 y calcular la subsidencia o el levantamiento para algún tiempo particular ( $Y_{i}$ en la Figura 6.2).

En la Figura 6.2b se muestra la curva de subsidencia tectónica de una cuenca de tipo rift, en la que se observa un evento rápido (syn-rift) seguido de un largo período (de 50 a $100 \mathrm{Ma}$ ) de una subsidencia tipo exponencial (post-rift) [20].

La técnica de backstripping descripta está basada en el modelo de Airy. Cuando se aplica a datos provenientes de un perfil de pozo es probable que resulte más exacta (1D). Sin embargo su alcance es limitado, dado que no provee información de la subsidencia tectónica o levantamiento más allá de las cercanías del pozo. Una solución para este problema es el backstripping flexural. En esta forma de backstripping, los datos de espesor sedimentario, determinados de un perfil de reflexión sísmica, se usan para determinar la subsidencia tectónica a lo largo de un perfil.

El backstripping flexural sigue un procedimiento similar al de Airy excepto que los sedimentos están ahora flexuralmente descargados desde la litosfera. Considerando el caso especial de la deflexión de una placa elástica sobre un sustrato dúctil causada por una carga periódica en la que a) la longitud de onda de la carga es infinitamente larga $\left(k \rightarrow 0\right.$ cargas anchas, ec. 5.28 del Cap. 5) y b) en donde la densidad de la corteza $\left(\rho_{c}\right)$ es reemplazada por la densidad de carga $\left(\rho_{l}\right)$, se tiene:

$$
w \rightarrow \frac{\left(\rho_{l}-\rho_{w}\right) h}{\left(\rho_{m}-\rho_{\text {relleno }}\right)}
$$

donde $h$ es la altura de la carga, $w$ es la flexura, $\rho_{l}$ es la densidad de la carga, $\rho_{w}$ es la densidad del material desplazado por la carga, $\rho_{\text {relleno }}$ es la densidad del material de relleno de la flexura y $\rho_{m}$ es la densidad del manto.

Reemplazando en la ec. $6.7 S^{*}$, que es el espesor total de sedimentos para un tiempo particular, $\rho_{l}$ por $\rho_{s}$ (la densidad de la secuencia) y asumiendo que $\rho_{\text {relleno }}$ es $\rho_{w}$ en vez de $\rho_{s}$.

la ecuación 6.7 queda

$$
w \rightarrow \frac{\left(\rho_{s}-\rho_{w}\right) S^{*}}{\left(\rho_{m}-\rho_{w}\right)}
$$


a)

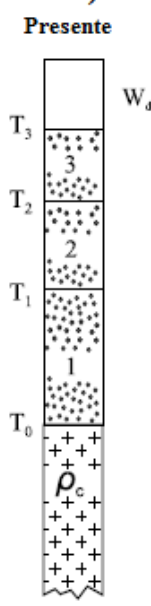

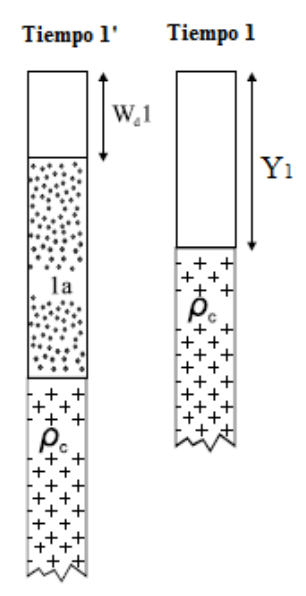

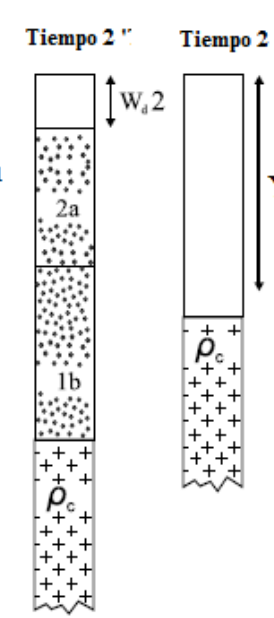

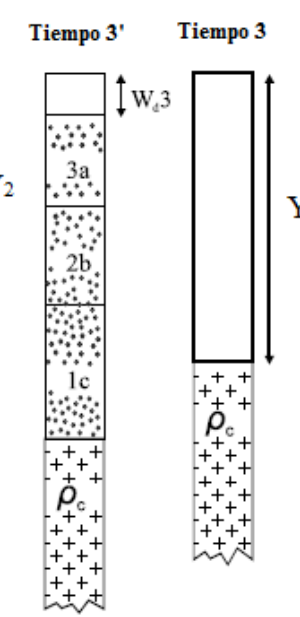

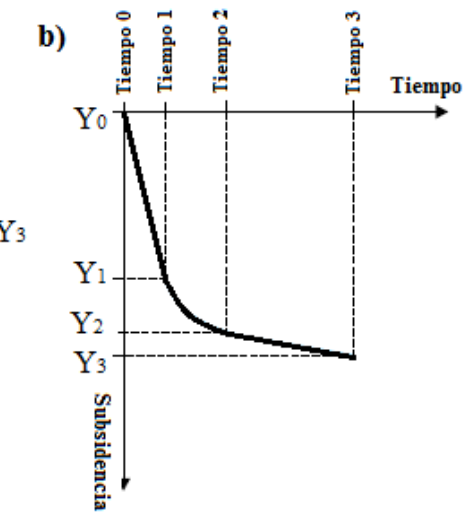

Figura 6.2. Backstripping multicapa. En a) la primera columna representa lo observado (al presente) totalmente cargado por una secuencia de 3 unidades. Al Tiempo1', la Unidad 1 es descompactada a su posición original y su densidad (ec. 6.1 y 6.2). Al Tiempo 1 se restablece isostáticamente la profundidad del basamento en ausencia de la Unidad 1 (backstripping, ec. 6.5), recobrando la subsidencia Y1 al tiempo paso 1. Al Tiempo 2' el espesor de la Unidad 2 es decompactado y la Unidad 1 es compactada de acuerdo a la nueva profundidad. Al Tiempo 2 la Unidad 2 es descompactada totalmente y la Unidad 1 es compactada parcialmente, luego se aplica el backstripping recobrando la subsidencia Y2 al tiempo 2. Este procedimiento es repetido para la capa 3 . En b) se dibuja la correspondiente curva de subsidencia Y, modificado de Cuna [20]. 


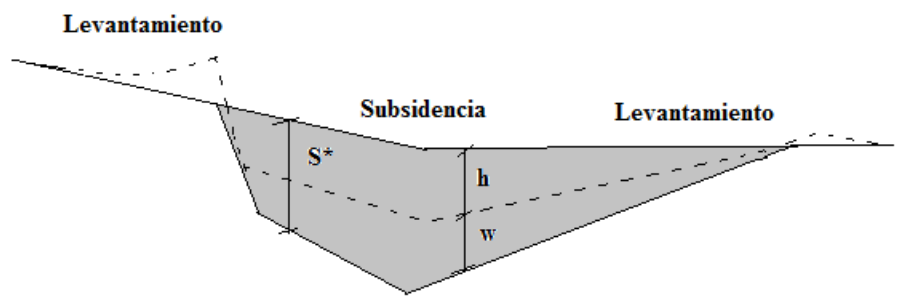

Figura 6.3. Los principios del backstripping flexural. La región sombreada muestra la cuña sedimentaria. La línea de guiones intermitentes representa la posición que tendría el basamento en ausencia de la carga de sedimentos. Modificado de Watts (2001) [103].

siendo $S^{*}=w+h$ (Fig. 6.3) se sigue:

$$
h \rightarrow \frac{\left(\rho_{m}-\rho_{s}\right) S^{*}}{\left(\rho_{m}-\rho_{w}\right)}
$$

que es el backstripping de Airy, la ecuación 6.5 con la profundidad del agua igual a 0 y sin cambios del nivel del mar.

El backstripping flexural calcula en primer lugar la flexura debido a la carga total de sedimentos con $\rho_{\text {relleno }}=\rho_{w}$ y luego se resta la flexura resultante, $w$, del espesor observado, $S^{*}$, para obtener la subsidencia tectónica y el levantamiento. La subsidencia tectónica es la parte de la subsidencia de un margen no causada por el peso de los sedimentos y carga de agua a través del tiempo [97].

Se aplica transformada de Fourier a la ecuación 6.8:

$$
\mathbf{W}(k)=\frac{\left(\rho_{s}-\rho_{w}\right) \mathbf{S}^{*}(k)}{\left(\rho_{m}-\rho_{w}\right)} \phi_{e}^{\prime \prime}
$$

donde $\mathbf{W}(k)$ es la transformada de Fourier de $w(x), \mathbf{S}^{*}(k)$ es la transformada de Fourier del espesor $S^{*}(x)$ y $\phi_{e}^{\prime \prime}=\left[\frac{D K^{4}}{\left(\rho_{m}-\rho_{w}\right) g}+1\right]^{-1}$ es el parámetro flexural que multiplicado por la respuesta de Airy da la flexura. El backstripping $h(x)$ (ver Fig. 6.3) puede calcularse con

$$
h(x)=S^{*}-w(x)
$$

En la práctica, el backstripping se calcula capa por capa con la opción de asignarles densidades y un $T_{e}$ diferentes. El backstripping flexural para un tiempo en particular, se obtiene sumando los efectos de cada capa, empezando por la primer capa.

\subsubsection{Subsidencia tectónica y Factor de estiramiento}

\section{Modelo de cuenca de rift de McKenzie}

La subsidencia tectónica (TS, por sus siglas en inglés) en zonas extensionales, como son las cuencas de rift intracontinentales o los márgenes continentales pasivos, se describe como el desplazamiento vertical del basamento causado por re-equilibrios isostáticos y termales de la litosfera continental estirada $[83,59,85]$. De acuerdo al modelo de rift de 
McKenzie [59], la historia de subsidencia/levantamiento de una cuenca de rift sedimentaria se compone de dos etapas. La primera etapa corresponde a un rápido movimiento ascendente/descendente del basamento, que resulta de un re-equilibrio isostático de las masas en la región de la cuenca producto del rift. La segunda etapa consiste de un patrón de subsidencia asociado con la relajación termal de la litosfera, que progresivamente recobra el espesor de equilibrio (es decir el de la pre-extensión). Este esquema de formación y evolución de la cuenca de rift se muestra en la Figura 6.4 [20].

A pesar de no considerar otros efectos de los procesos de rift como son mezcla parcial con el manto y migración lateral de las capas débiles de la corteza inferior, el modelo tipo McKenzie explica muchas de las observaciones relacionadas con la formación y evolución de las cuencas sedimentarias de tipo rift, como la subsidencia y estructuras termales, espesor sedimentario y grandes patrones estratigráficos (ej. [59, 98]). Además, dichas observaciones permiten establecer una relación simple entre la cantidad de estiramiento $\beta$, la TS y el flujo de calor observado en una cuenca de rift [59].

Considerando algun espesor inicial de corteza, $T_{c}$, y de la litosfera, $L$, un gradiente geotermal de la litosfera (que incluye a la corteza) en estado estacionario y un manto sublitosférico isotermal [103], si la temperatura de la superficie de la litosfera es $0^{\circ} \mathrm{C}$ y la temperatura en su base es $t_{m}$ entonces la temperatura promedio de la base de la corteza está dada por [103]:

$$
\frac{t_{m} T_{c}}{2 L}
$$

similarmente, la temperatura promedio en el manto sub cortical es [103]:

$$
\frac{\left(\left(t_{m} T_{c}\right) / L\right)+t_{m}}{2}=\frac{t_{m}\left(T_{c}+L\right)}{2 L}
$$

La subsidencia inicial o isostática de una cuenca de rift puede calcularse por balance de masas de las columnas de la litósfera normal y adelgazada bajo un dado nivel de referencia (ver Figura 6.4a y b, respectivamente). La presión en la base de la litósfera antes de que el estiramiento haya ocurrido $P_{a n t}$ es dado por:

$$
P_{\text {ant }}=T_{c} \bar{\rho}_{c} g+\left(L-T_{c}\right) \bar{\rho}_{m} g
$$

donde L y $T_{c}$ son el espesor de la litósfera sin estirar y la corteza , respectivamente. Las densidades del manto y la corteza, $\bar{\rho}_{m}$ y $\bar{\rho}_{c}$ son valores promedio que dependen de la temperatura y son dados por[20]:

$$
\begin{gathered}
\bar{\rho}_{c}=\rho_{0 c}\left[1-\alpha \frac{t_{m} T_{c}}{2 L}\right] \\
\bar{\rho}_{m}=\rho_{0 m}\left[1-\alpha \frac{t_{m}}{2}\left(1+\frac{T_{c}}{L}\right)\right]
\end{gathered}
$$

donde $\rho_{0 c} \mathrm{y} \rho_{0 m}$ son las densidades de la corteza y el manto a $0^{\circ} \mathrm{C}$, respectivamente, $\alpha$ es el coeficiente volumétrico de expansión termal.

Inmediatamente después de que el rift se ha creado, la presión al nivel de referencia de la litosfera adelgazada, $P_{\text {des }}$ es dado por (Figura 6.4b),

$$
P_{\text {des }}=S_{i} \rho_{w} g+\frac{T_{c}}{\beta} \bar{\rho}_{c} g+\left(\frac{L-T_{c}}{\beta}\right) \bar{\rho}_{m} g+\left(L-S_{i}-\frac{L}{\beta}\right) \rho_{a} g
$$



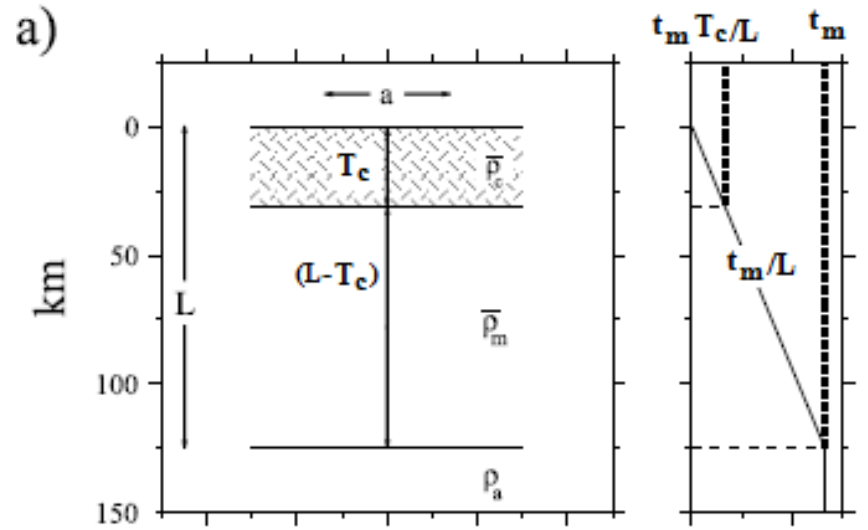

b)
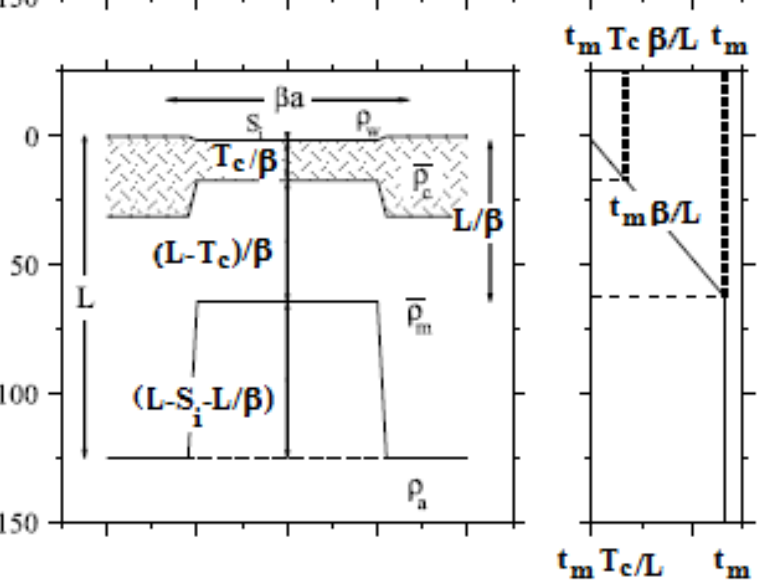

c)
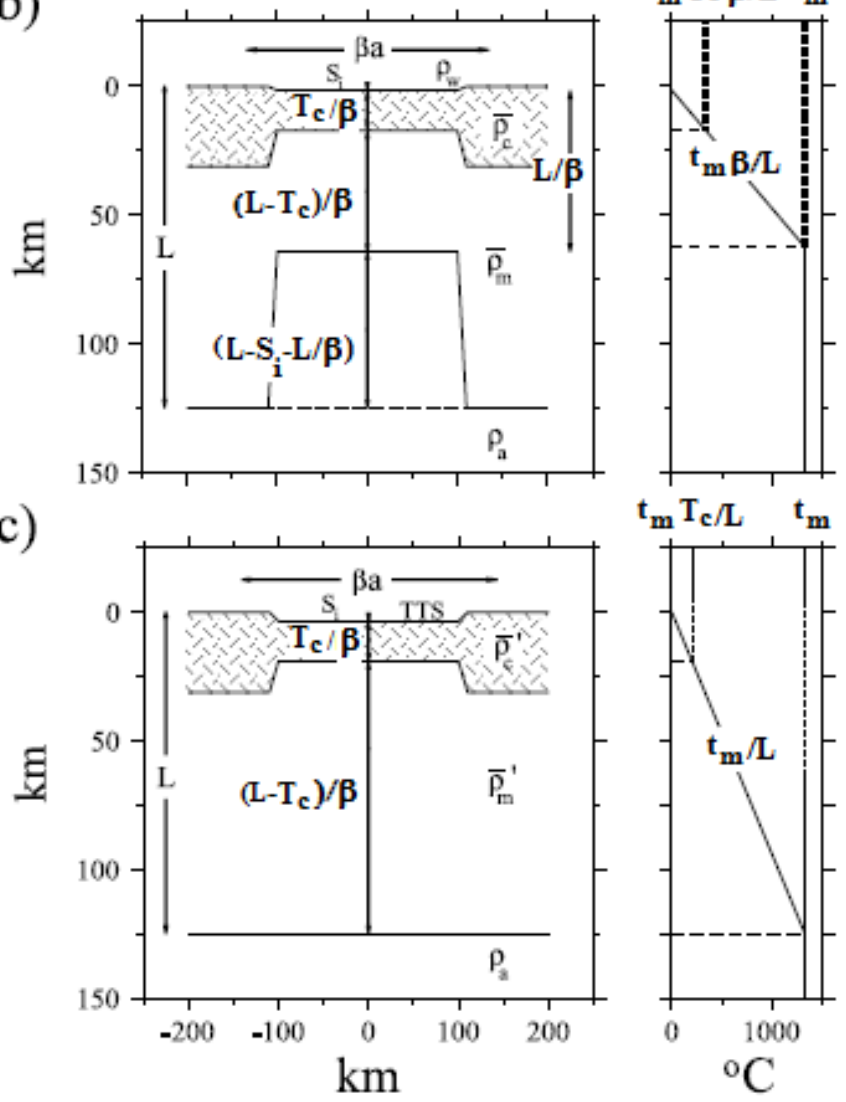

Figura 6.4. Dos etapas del modelo propuesto por McKenzie [59] para explicar la formación y evolución de cuencas de rift: subsidencia (izquierda) y estructura termal de la litosfera (derecha) a) litosfera pre estiramiento. b) Despúes de un evento de rift instantáneo. c) Después de la relajación termal de la litosfera y recupero del gradiente de la temperatura inicial. $T_{c}$ espesor inicial de corteza, L espesor de la litosfera, $t_{m}$ es la temperatura de la base de la litosfera, $S_{i}$ es la subsidencia inicial de la cuenca, $S_{f}$ es la subsidencia final de la cuenca, $\beta$ es el factor de estiramiento, TTS subsidencia tectónica total, $\rho_{m}$ y $\rho_{c}$ son valores promedio de densidades del manto y de la corteza previos e inmediatamente posteriores al estiramiento, $\rho_{m}^{\prime} \mathrm{y}$ $\rho_{c}^{\prime}$ son valores promedio de densidades del manto y de la corteza posteriores al estiramiento cuando se han alcanzado condiciones de equilibrio. Modificado de Cuna [20]. 
donde $\rho_{a}$ es la densidad de la astenósfera y $S_{i}$ es la subsidencia inicial de la cuenca. Hemos considerado aquí el caso de estiramiento instantáneo donde las densidades promedio de la corteza y manto litosférico son similares antes y después del estiramiento. Igualando las ecuaciones 6.14 y 6.16 se llega a:

$$
S_{i}=L\left(1-\frac{1}{\beta}\right) \frac{\left[\left(\rho_{0 m}-\rho_{0 c}\right) \frac{T_{c}}{L}\left(1-\frac{\alpha t_{m} T_{c}}{2 L}\right)-\frac{\rho_{0 m} \alpha t_{m}}{2}\right]}{\left[\rho_{0 m}\left(1-\alpha t_{m}\right)-\rho_{w}\right]}
$$

Después de un largo periodo de tiempo, el gradiente de la anomalía termal generado por el estiramiento se relajará (Figura 6.4c). Dado que la densidad media de la astenosfera es menor que la de la litosfera $\left(\rho_{a}=\rho_{0 m}\left[1-\alpha t_{m}\right]\right)[20]$, la relajación termal resultará en una mayor subsidencia de la cuenca. La presión en el nivel de referencia luego de que la litosofera ha recobrado su espesor de equilibrio $P_{e q}$ es dada por,

$$
P_{e q}=S_{f} \rho_{w} g+\frac{T_{c}}{\beta} \bar{\rho}_{c}^{\prime} g+\left(L-S_{f}-\frac{T_{c}}{\beta}\right) \bar{\rho}_{m}^{\prime} g
$$

donde $S_{f}$ es la subsidencia final de la cuenca, o subsidencia tectónica total (TTS, por sus siglas en inglés), que resulta del estiramiento y la relajación termal.

De la Figura 6.4c es evidente que la geoterma inicial antes de que el estiramiento haya ocurrido se restablece. Sin embargo, las proporciones relativas de la corteza y el manto en la litosfera son ahora diferentes, consecuentemente, las expresiones para las densidades promedio de la corteza y del manto se han alterado [20],

$$
\begin{gathered}
\bar{\rho}_{c}^{\prime}=\rho_{0 c}\left[1-\alpha \frac{t_{m} T_{c}}{2 \beta L}\right] \\
\bar{\rho}_{m}^{\prime}=\rho_{0 m}\left[1-\alpha \frac{t_{m}}{2}\left(1+\frac{T_{c}}{\beta L}\right)\right]
\end{gathered}
$$

Igualando las ecuaciones 6.14 con 6.18 y resolviendo para TTS se encuentra,

$$
T T S=\frac{L\left(\bar{\rho}_{m}^{\prime}-\rho_{m}\right)+T_{c}\left(\rho_{m}+\frac{\bar{\rho}_{c}^{\prime}}{\beta}-\frac{\bar{\rho}_{m}^{\prime}}{\beta}-\rho_{c}\right)}{\left(\bar{\rho}_{m}^{\prime}-\rho_{w}\right)}
$$

El modelo de McKenzie permite explicar la formación de un rift de manera simple: después de un evento de rift instantáneo, se produce la relajación termal de la litosfera, y posteriormente se vuelve al equilibrio inicial. Este proceso ha creado una "cubeta" con una subsidencia tectónica total y una elevación del manto por compensación isostática. En este trabajo de tesis explicamos el modelo, pero no lo utilizamos porque partimos de la cuenca ya desarrollada y la descargamos con el método de backstripping, que permite obtener una estimación de la subsidencia tectónica total.

\section{Factor de estiramiento $\beta$, según Airy}

Una definición sencilla del factor de estiramiento o factor $\beta$ es decir que es el cociente entre el grosor de la corteza inicial sobre el grosor de la corteza adelgazada. Un factor $\beta$ de 1.3 generará una subsidencia de $3 \mathrm{~km}$. Para un proceso de rifting completo (Formación de corteza oceánica y cuenca oceánica) el factor $\beta$ tiende a infinito 
(www.le.ac.uk/geology/art/gl209/lecture4/lecture4.html).

En una cuenca de tipo rift el valor de $\beta$ da la cantidad de adelgazamiento y, por lo tanto, la cantidad de calor que se ha generado, lo que permite estimar el espesor de la corteza. A modo de ejemplo, el $\beta$ que mejor ajusta a los datos del backstripping en función del tiempo puede usarse para estimar el adelgazamiento de la corteza y la estructura de la corteza.

La estructura de la corteza puede estimarse desde el backstripping, sin embargo debemos hacer alguna consideración acerca del estado de isostasia durante el proceso de rift [103]. De acuerdo a Airy, el backstripping será localmente compensado por variaciones en el espesor de la corteza. Por lo tanto regiones donde hay subsidencia tectónica se asociarán con regiones de corteza delgada y donde hay levantamiento tectónico con regiones de corteza de gran espesor. Podemos estimar la cantidad de adelgazamiento o engrosamiento considerando la presión en la base de una columna antes y después del adelgazamiento (ver Fig. 6.5).

$$
T_{c} \rho_{c} g=Y \rho_{w} g+T_{s} \rho_{c} g+x \rho_{m} g
$$

donde

$$
x=T_{c}-T_{s}-Y
$$

Combinando las ecuaciones obtenemos:

$$
T_{s}=T_{c}-Y \frac{\left(\rho_{m}-\rho_{w}\right)}{\left(\rho_{m}-\rho_{c}\right)}
$$

por lo que

$$
\begin{gathered}
\beta=\frac{T_{c}}{T_{s}} \\
\beta=\frac{\left(\rho_{m}-\rho_{c}\right) T_{c}}{\left(\rho_{m}-\rho_{c}\right) T_{c}-Y\left(\rho_{m}-\rho_{w}\right)}
\end{gathered}
$$

siendo Y la TTS. En la perspectiva de esta tesis, y del POGM en general $[99,86]$ la TTS corresponde a la posición del basamento en ausencia de cargas de superficie (ej. sedimentarias, volcánicas) y de sub superficie (ej. subplacado) [20]. Por lo que una vez que se ha aplicado el backstripping a éstas cargas, la ecuación 6.25 puede usarse para recobrar la geometría de la cuenca al tiempo del rift que es definida por la TTS y el backstripping del Moho.

Se ha asumido que cuando la corteza sufre extensión se genera una depresión ocupada por agua que se compensa por el adelgazamiento de la corteza, como predice el modelo de Airy. El problema con esta suposición es que se ignora la resistencia finita de la litósfera. Varios investigadores [11] han señalado que zonas rígidas de la litosfera podrían modificar significativamente la estructura de una corteza extendida. Esto se debe a que la respuesta de una corteza sometida a extensión no es justamente un problema termal sino mecánico.

Se conoce a partir de resultados de experimentos mecánicos con rocas [103] que el esfuerzo que soportan los materiales que forman la corteza y el manto crece y luego decrece con la profundidad. Habrá una profundidad en la que el esfuerzo sea máximo en la litosfera y depende del gradiente geotermal, el espesor inicial de corteza y la velocidad de deformación. Cuando la litosfera es sometida a extensión, esperamos que las zonas 


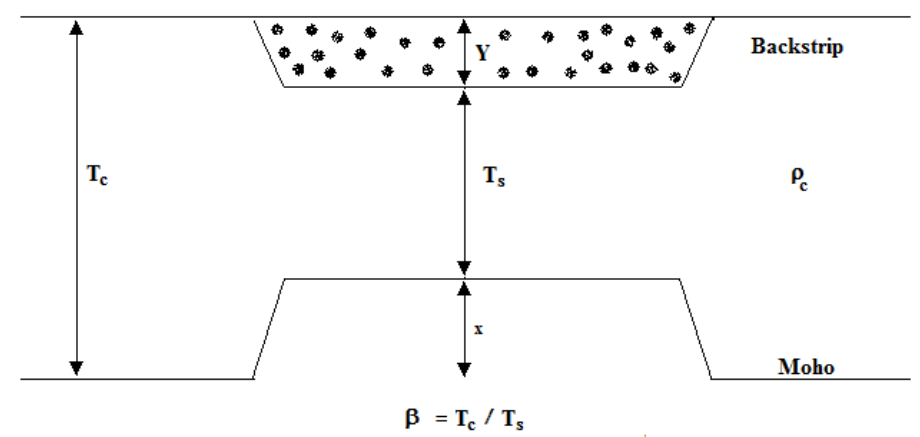

Figura 6.5. Relación entre el estiramiento y el espesor de la corteza.

de esfuerzo máximo actúen como zonas de resistencia que particionan verticalmente la deformación dentro de niveles de diferente profundidad en la corteza y el manto [103].

\subsubsection{Subplacado magmático}

Muchas cuencas de tipo rift están asociadas con magmatismo, quizás el mejor ejemplo lo constituyan las cuencas llamadas márgenes volcánicos del atlántico norte que han sido influenciadas por plumas. El magmatismo se expresa en perfiles de sísmica de reflexión [43] como una serie de reflectores sísmicos que inclinan hacia el mar: Seaward Dipping Reflectors (SDRs) y en datos de sísmica de refracción [28, 109, 50] como una inusual velocidad de onda $\mathrm{P}$ alta en cuerpos de la corteza inferior.

Los SRDs típicamente muestran un patrón de reflector que es dominado por un engrosamiento en profundidad. El patrón se asemeja a la geometría clinoform de grandes sistemas de ríos progradantes [103]. El analisis de los datos del Proyecto de pozos oceánicos (ODP) (ej. Lebombo, Mozambique $[19,103])$ sugiere que los SDRs comprenden gruesas secuencias basálticas [103]. De acuerdo a Mutter et al. [63, 103], los basaltos extrusivos representan lavas sub-aéreas. Si esto se considera correcto, la geometría sigmoidal sería probablemente una combinación de los efectos de la contracción termal y la flexura de la litosfera subplacada por la carga volcánica, cuando el margen se separa de la fuente principal del magma de la dorsal oceánica.

La estructura de velocidad de onda $\mathrm{P}$ de los cuerpos de la corteza inferior, que no han sido perforados, sugiere que están compuestos de material magmático subplacado en la corteza. El origen del material magmático no se conoce, pero muchos investigadores han sugerido [103] que se genera a gran profundidad en el manto, que ha ascendido debido a su flotabilidad (relativa diferencia de densidad entre el magma ascendente y las rocas de los alrrededores) y que ha quedado atrapado cerca del Moho. El subplacado magmático puede causar el engrosamiento de la corteza cuando el magma se enfría. Xenolitos de material del manto puede darnos información sobre la última fuente del magma y revelar heterogeneidades de la mezcla de magma y asimilación de magmas en profundidad [6]. Donde se tiene perfiles sísmicos, la corteza entre los SDRs y los cuerpos de la corteza inferior es extremadamente delgada, y al menos localmente se atribuye al estiramiento de la corteza continental.

Las primeras pruebas de la presencia de material subplacado por debajo del Moho continental estaban basadas en la adquisición de datos de sísmica y gravedad de ba- 


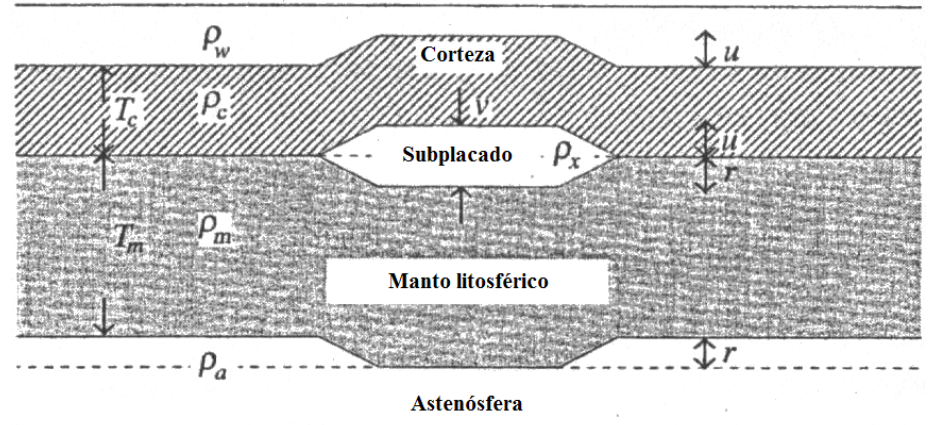

Figura 6.6. Modelo simple de subplacado magmático de la corteza de espesor uniforme. Modificado de Watts [103].

ja resolución y los resultados estaban en general de acuerdo con modelos de grandes capas continuas de material subplacado [28]. Recientes experimentos sísmicos [31, 32] de mayor resolución han mejorado la estructura de imágenes de material subplacado e intrusiones maficas en la corteza continental y han posibilitado el avance en la comprensión general de los procesos involucrados [88]. El backstripping en margenes volcánicos muestra que la subsidencia tectónica tiene generalmente una forma similar a la de los márgenes no volcánicos, aunque más pequeña en magnitud [15]. Esto es totalmente esperable dado que los SDRs y el subplacado engrosan la corteza y causan un levantamiento.

El subplacado perturba el estado de la isostasia en la región. Podemos estimar la cantidad de levantamiento que resultaría del equilibrio comparando columnas de corteza subplacadas con las que no lo están [103].

$$
T_{c} \rho_{c}+u \rho_{w}+T_{m} \rho_{m}+\rho_{a} r=T_{c} \rho_{c}+\nu \rho_{x}+T_{m} \rho_{m}
$$

donde $u$ es el levantamiento, $\nu$ es el espesor del subplacado, $r=\nu-u$, $T_{c}$ es el espesor de la corteza y $T_{m}$ es el espesor de la litosfera sub cortical (ver Fig. 6.6).

$$
\begin{gathered}
u \rho_{w}+\rho_{a} r=\nu \rho_{x} \\
u \rho_{w}+\rho_{a}(\nu-u)=\nu \rho_{x} \\
u=\nu \frac{\left(\rho_{a}-\rho_{x}\right)}{\left(\rho_{a}-\rho_{w}\right)}
\end{gathered}
$$

Se observa en la ecuación 6.27 que si $\nu=15 \mathrm{~km}, \rho_{a}=3200 \mathrm{kgm}^{-3}$ y $\rho_{w}=1030 \mathrm{kgm}^{-3}$ se obtiene un $\mathrm{u}=2.41 \mathrm{~km}$.

La ecuación 6.29 asume una repuesta de la corteza tipo Airy al subplacado. Podemos calcular los efectos flexurales definiendo el parámetro número de onda apropiado que modifica la respuesta de Airy al que produce la flexura:

$$
\mathbf{U}(k)=\mathbf{V}(k) \frac{\left(\rho_{a}-\rho_{x}\right)}{\left(\rho_{a}-\rho_{w}\right)} \phi_{e}(k)
$$

donde $\mathbf{U}(k)$ y $\mathbf{V}(k)$ son la transformada de Fourier del levantamiento y del espesor del subplacado, respectivamente, y $\phi_{e}$ el parámetro número de onda, que es dado por:

$$
\phi_{e}(k)=\left[1+\frac{D k^{4}}{\left(\rho_{a}-\rho_{w}\right) g}\right]^{-1}
$$


El subplacado magmatico implica una redistribución de masas que debería estar asociada con las anomalías de gravedad. Por ejemplo, el subplacado en el talud continental sin resistencia a la flexión ensancha el bajo del efecto de borde típico de la anomalía de aire libre y reduce su amplitud [101]. Si la corteza tiene rigidez, el bajo se estrecha y la amplitud se incrementa [102]. La anomalía del subplacado depende fuertemente de la resistencia de la litosfera. En un margen débil $\left(T_{e}=0 \mathrm{~km}\right)$ el subplacado reduce la amplitud del "alto" y los flancos "bajos" e incrementa su longitud de onda. En un margen fuerte $\left(T_{e}=20 \mathrm{~km}\right)$ ocurre el efecto opuesto el subplacado incrementa la amplitud del alto y bajos y decrece su longitud de onda [102]. La extensión del subplacado debería revelarse en la anomalía isostática de Airy, además de identificarse en la corteza con alta rigidez flexural.

Respecto a la extensión del subplacado en el margen de rift, algunos márgenes, especialmente los del norte del Atlántico han sido influenciados por la pluma de Islandia y son fuertemente magmáticos. Esto queda revelado por el escaso sedimento que cubre a éste tipo de márgenes. Otros, sin embargo tienen abundante magmatismo y son caracterizados por espesas secuencias sedimentarias [103].

\subsection{Modelado de Gravedad Orientado por los Pro- cesos}

Una vez determinado el backstripping se puede estimar el espesor de la corteza sometida a extensión. El principal problema es que no conocemos el verdadero valor del espesor elástico $\left(T_{e}\right)$ para usar en el backstripping de sedimentos y poder restablecer la configuración de la corteza al tiempo del rift.

Un parámetro que puede usarse para restringir los valores de $T_{e}$ es la anomalía de gravedad. La anomalía de gravedad en un rift es sensible a la estructura de la corteza inicial y a procesos como sedimentación, subplacado magmático y erosión. Calculando la anomalía de gravedad causada por la estructura inicial del rift y la asociada a los procesos modificadores y comparando con la anomalía observada, se obtiene un procedimiento que puede restringir los valores de $T_{e}$. Watts [102] sugirió llamar a este procedimiento "procesos orientados", que es una técnica basada en tres pasos:

1) Se obtiene la anomalía de gravedad debida al backstripping, de una cuenca ocupada por agua y se calcula su compensación. Esta anomalía se conoce como anomalía de rift.

2) Se obtiene la anomalía de gravedad debida a la carga de sedimentos y se calcula su compensación. Esta anomalía es conocida como anomalía de sedimentación. La diferencia entre el resultado del backstripping ocupado por agua y la topografía al presente da la carga de los sedimentos, la anomalía de los sedimentos se calcula desde la carga de los sedimentos.

3) El paso final es calcular la anomalía suma debida al rift y a la sedimentación y compararla con la anomalía observada sobre la cuenca de rift.

Los márgenes continentales pueden ser segmentados con respecto a su rigidez flexural, lo que puede en parte, explicar las variaciones de las anomalías de gravedad que se han observado en algunos márgenes (ej: Gabon/Angola; [100]).

El Modelado de Gravedad Orientados por los Procesos [102, 103] combina la técnica 
backstripping (sección 6.1.1) con técnicas de modelado de gravedad. El backstripping es una técnica cuantitativa basada en la remoción de carga desde el basamento, aplicada bajo la hipótesis de Airy o flexural, que asume que se conoce la carga producida por el agua y por los sedimentos. De la ecuación del backstripping ([103]), ecuación 6.5 es posible determinar la subsidencia tectónica directamente desde datos estratigráficos. Dicha ecuación consta de tres términos: el primer término es la profundidad del agua, el segundo representa la carga de los sedimentos y el tercero es la carga del nivel del mar.

Inicialmente aplicamos la técnica backstripping a perfiles, con ésta técnica podemos estimar la profundidad del basamento en ausencia de sedimentos, es decir la paleobatimetría en el tiempo del rift, la cual ha sido calculada teniendo en cuenta todo el espesor de los sedimentos sin diferenciación de edades. Para el caso del backstripping flexural se determina el parámetro apropiado en el espacio de los números de onda, que modifica la respuesta de Airy produciendo la respuesta flexural, resultante de una ecuación equivalente a la ecuación 6.5 en el dominio de Fourier.

El POGM utiliza información sísmica del espesor sedimentario y del volcanismo presente (SDRs, suplacado, ect.) de la configuración del margen actual, para estimar la anomalía de gravedad que está asociada a los procesos: sedimentación y magmatismo (no considerado en ésta tesis). El objetivo del POGM es discriminar la contribución de los diferentes procesos geológicos a la anomalía observada y el del backstripping determinar la geometría del margen al tiempo del rift.

Podemos resumir los pasos para aplicar el POGM:

- 1) Se aplica el backstripping a la carga de sedimentos para determinar el basamento al tiempo del rift.

- 2) Se calcula el factor de estiramiento $\beta$, según Airy, para restablecer la geometría del Moho al tiempo del rift.

- 3) Se calcula la anomalía de gravedad asociada con el rift. Para algunos perfiles dicha anomalía muestra el típico efecto de borde con un alto sobre la plataforma continental y un bajo en la región del talud y para otros revela complejidades que no siguen ese patrón.

- 4) Se restablece la carga de los sedimentos al margen y se calcula la flexura del basamento para la estructura de espesores elásticos $\left(T_{e}\right)$ utilizada en el paso 1) y por consiguiente la del Moho.

- 5) Se calcula la anomalía asociada con los procesos de la sedimentación: carga de los sedimentos y flexura del basamento y del Moho.

- 6) Finalmente se obtiene la anomalía suma (anomalía calculada) dada por la adición de las anomalías del rift y la de la sedimentación y se compara con la anomalía observada (go), lo que representa la principal restricción para el parámetro $T_{e}$. Aqui debiera también calcularse la anomalía del suplacado como componentes de la anomalia suma pero queda fuera del aclanze de esta tesis. 


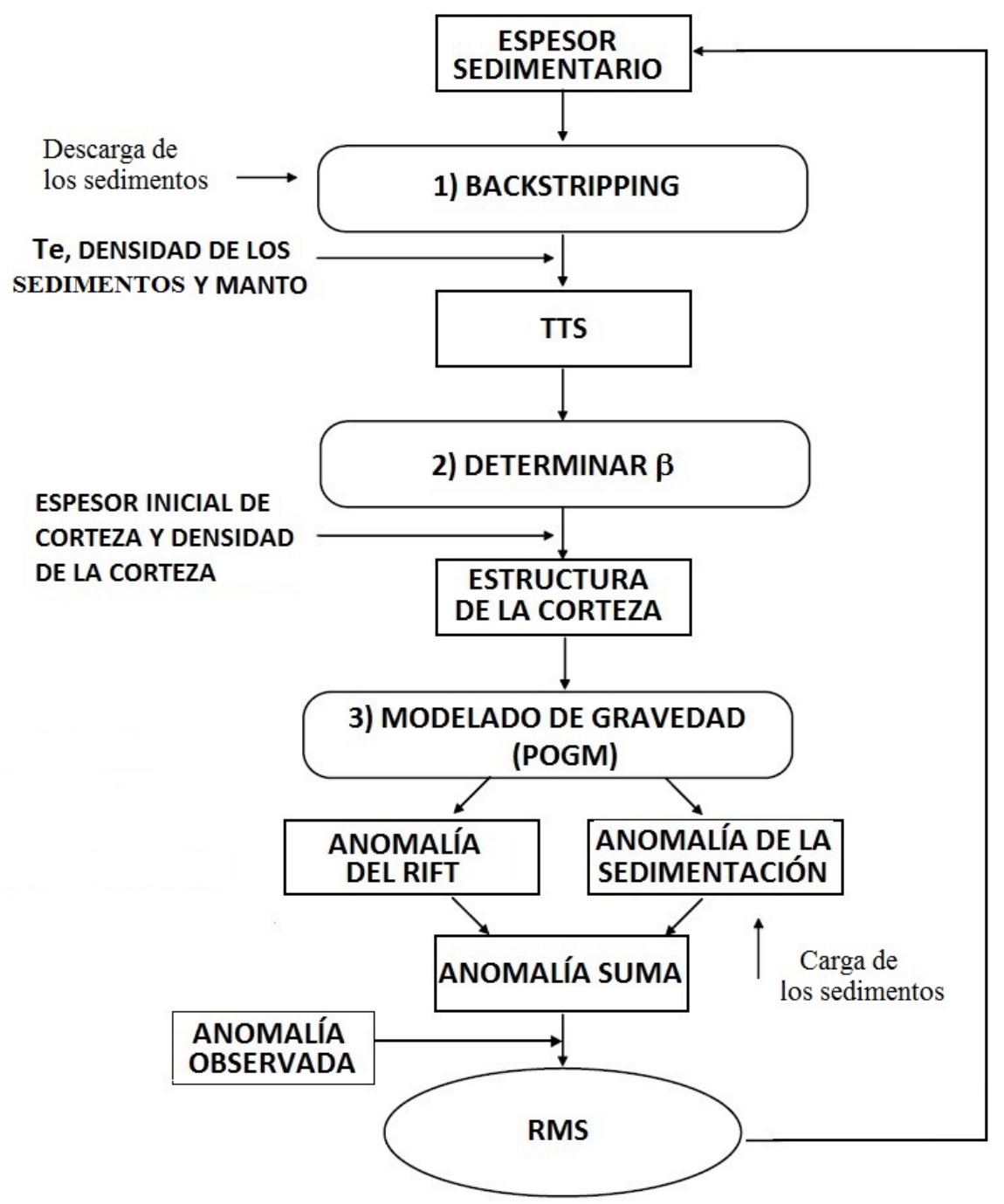

Figura 6.7. Diagrama de flujo para aplicar el POGM adaptado de Cuna [20] y Stewart et al. [86].

\subsection{Aplicación de las técnicas Backstripping y POGM}

Para la aplicación de la técnica backstripping se ignoran los efectos de la carga de agua debido a cambios del nivel del mar. Siguiendo los pasos descriptos en la sección 6.2, se generó un programa de computación que permite aplicar las técnicas backstripping y POGM en la región del margen. Para el caso de perfiles 2D, puede observarse en la Figura 6.7 un diagrama de flujo con los principales pasos: El programa de computación creado, tiene por objeto automatizar el cálculo del backstripping y el POGM a perfiles (2D) y luego extender a grillas (3D). ho al tiempo del rift. Una vez que contamos con las topografías de la paleobatimetría y el paleoMoho ensayamos aplicar la carga sediemntaria y modelar la flexura de la corteza y del Moho que nos darían la ubicación de estas topografías en la actualidad.

Las interfaces y sus contribuciones gravitacionales de un modelo sintético pueden observarse en la Figura 6.8 . 

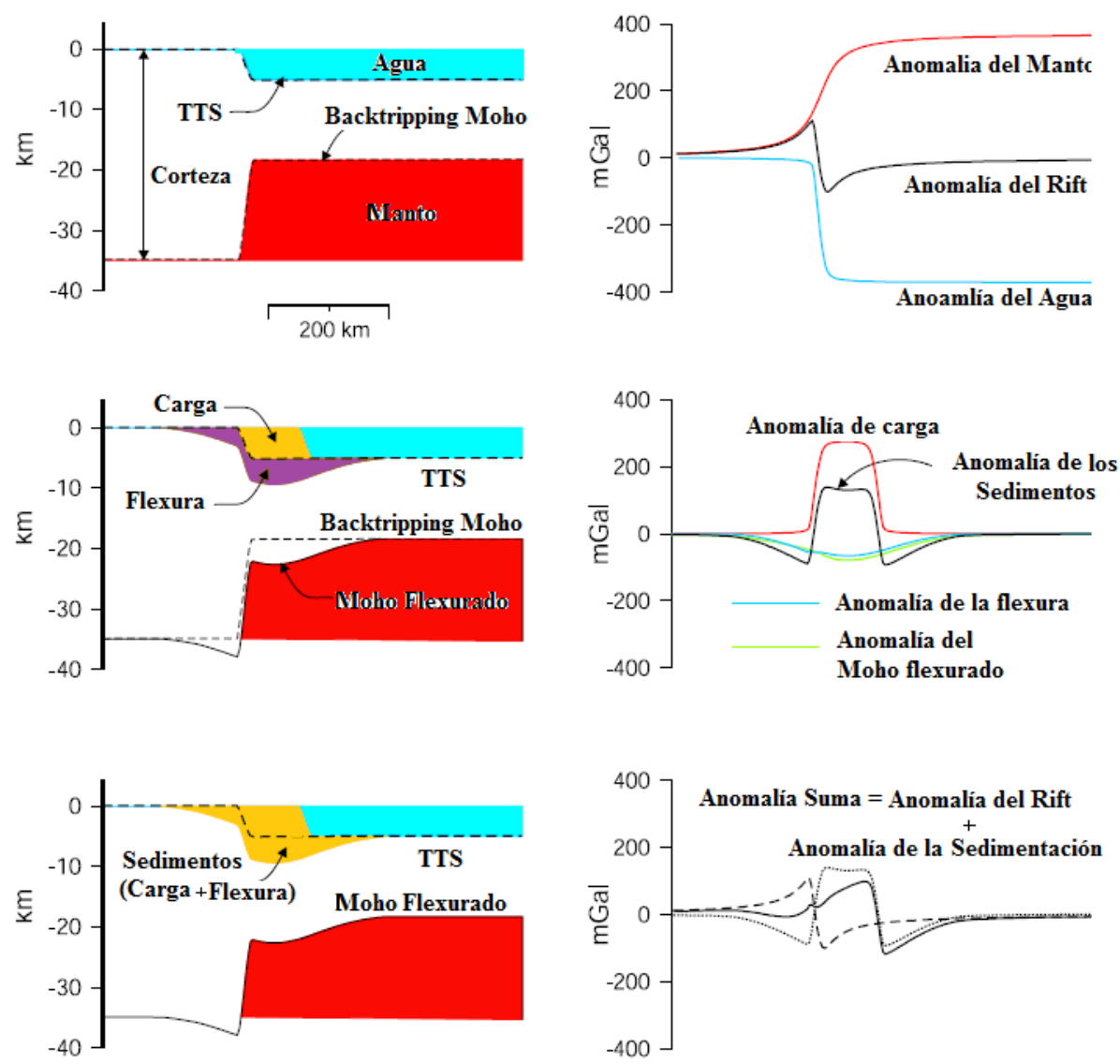

Figura 6.8. Modelado de Gravedad Orientado por los Procesos: diagrama sintético. Modificado de Cuna [20]. 


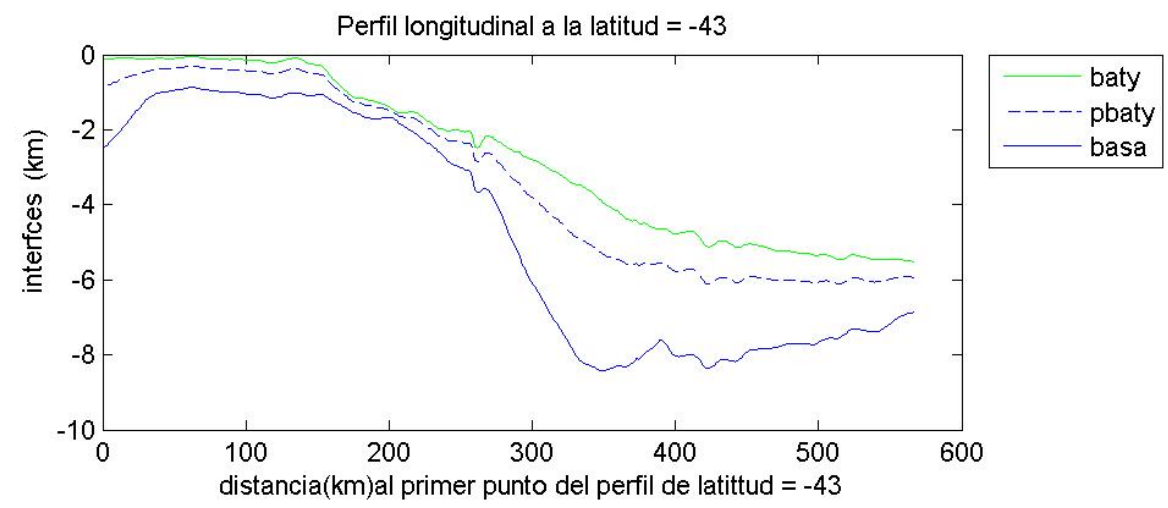

Figura 6.9. Backtripping para el caso perfil $43^{\circ} \mathrm{S}$ y un $T_{e}=10 \mathrm{~km}$. baty: batimetría, pbaty: paleobatimetría y basa: basamento.

\subsubsection{Aplicación 2D}

\section{1) Aplicar el backstripping a la carga de sedimentos}

Se generan arbitrariamente una serie de perfiles longitudinales separados por un dado espaciamiento en latitud a elección cubriendo todo el margen, luego se interpola para cada perfil en las grillas de batimetría, espesor sedimentario (compilado a partir de las fuentes mencionadas anteriormente) y anomalía de gravedad observada. Para resolver la ecuación del backstripping en el dominio de la frecuencia se consideró apropiado extender a la derecha los perfiles que están expresados en distancia al primer punto del perfil de oeste a este. Para resolver la ecuación del backstripping es necesario construir el parámetro número de onda k.

Se calcula el parámetro o filtro $\phi_{k}$ y se resuelve la ecuación del backstripping flexural para una serie de espesores elásticos en el que para el caso $T_{e}=0$ se tiene el caso del backtripping de Airy.

Hemos considerado una sola capa de sedimentos de una densidad promedio dado que contamos con una grilla de espesor sediemntario sin difernciacion de litología y no hemos descompactado la capa (estamos balanceando la columna a) con la c) de la Figura 6.1.

Se resuelve la ecuación:

$$
\begin{gathered}
\mathbf{W}(k)=\frac{\left(\rho_{s}-\rho_{w}\right) \mathbf{S}^{*}(k)}{\left(\rho_{m}-\rho_{w}\right)} \Phi_{e} \\
\Phi_{e}=\left[\frac{D k^{4}}{\left(\rho_{m}-\rho_{w}\right) g}+1\right]^{-1}
\end{gathered}
$$

donde en $\Phi_{e}, \rho_{\text {relleno }}$ es $\rho_{w}$ que indica que al descargar flexuralmente rellenamos el espacio vacante con agua. En la Figura 6.9 puede observarse el resultado de aplicar la técnica backstripping para obtener la paleobatimetría como la suma del backstripping y la batimetría actual para un espesor elastico de $T_{e}=10 \mathrm{~km}$. 


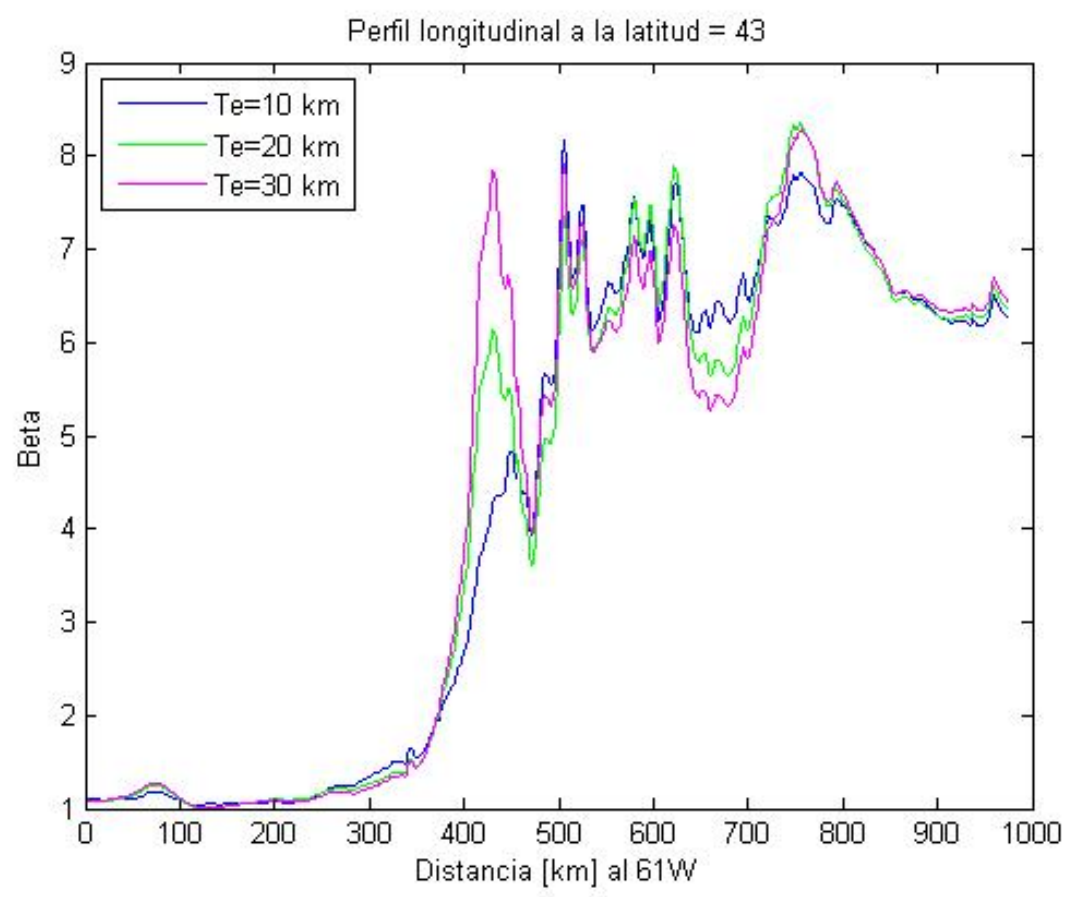

Figura 6.10. Factor de estiramiento $\beta$ para el caso perfil $43^{\circ} \mathrm{S}$.

\section{2) Calcular el factor de estiramiento $\beta$, según Airy}

Una vez obtenido el backstripping puede reconstruirse la paleobatimetría sumando el backstripping a la batimetría actual. En este paso en el que hemos reconstruído una estimación de la paleobatimetría al tiempo del rift se considera aplicar equilibrio isostático según Airy con una corteza de espesor normal de $32 \mathrm{~km}$, para calcular el parámetro $\beta$ (según Airy, Fig. 6.10) que nos permite encontrar la estructura de la corteza y por consiguiente la topografía del Moho al tiempo del rift. Una vez que contamos con las topografías de la paleobatimetría y el paleoMoho ensayamos aplicar la carga sedimentaria $(h(x)$, el backstripping o función de carga, la altura entre la paleobatimetría y la batimetría actual) y modelar la flexura de la corteza y del Moho que nos darían la ubicación de estas topografías en la actualidad (ver Fig. 6.3).

\section{3) Calcular la anomalía de gravedad asociada con el rift}

Para calcular la anomalía del rift contamos con dos superficies agua-corteza (paleobatimetría), corteza-manto (paleoMoho). Calculamos la gravedad (a partir de la ec. B.8 del Apéndice B ) asociada con dichos contrastes para aislar y obtener la anomalía del rift.

En la Figura 6.11 observamos un fuerte efecto de borde a la derecha, una zona de transición con tres máximos y un mínimo a la izquierda debido a una variación del espesor de la corteza continental que proviene de la cuenca de Rawson donde hay acortamiento cortical. Esto puede observarse también en los cálculos 3D. 


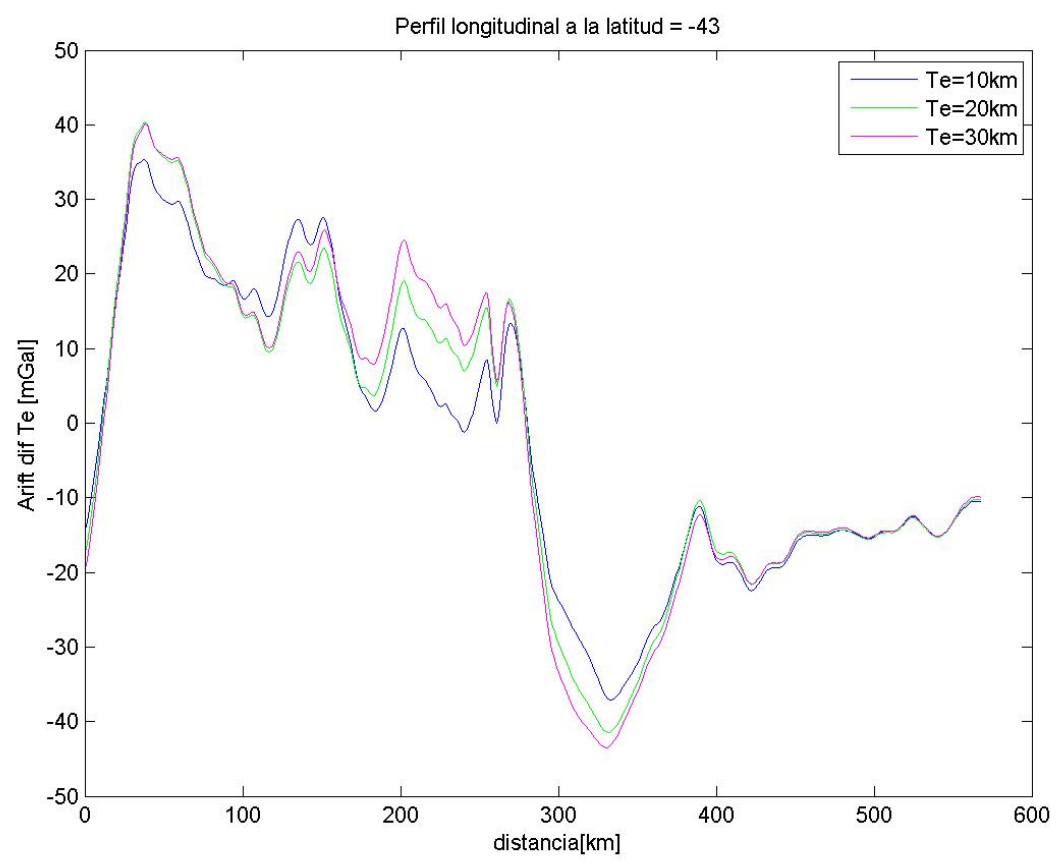

Figura 6.11. Anomalía del rift para el caso perfil $43^{\circ} \mathrm{S}$.

\section{4) Restablecer la carga de los sedimentos, calcular la flexura del basamento y del Moho}

$\mathrm{Al}$ considerar la flexura generada en la corteza y el manto por la carga de los sedimentos estamos, de alguna manera, perturbando el modelo de rift y esa perturbación puede identificarse en tres capas: la primera de ellas corresponde a una capa limitada en su base por la paleobatimetría y en su tope por la batimetría actual con contraste de densidad $(+)$ sedimento-agua, la segunda cuya base es el basamento y su tope es la paleobatimetría con contraste de densidad (-) sedimento-corteza, y la tercera tiene como base el Moho actual y tope el paleo-Moho con contraste de densidad cortezamanto (-). Son las mismas interfaces que las utilizadas en el modelo sintético (Fig. 6.8). Se resuelve la ecuación:

$$
\begin{gathered}
\mathbf{W}(k)=\frac{\left(\rho_{s}-\rho_{w}\right) \mathbf{H}(k)}{\left(\rho_{m}-\rho_{s}\right)} \Phi_{e} \\
\Phi_{e}=\left[\frac{D k^{4}}{\left(\rho_{m}-\rho_{s}\right) g}+1\right]^{-1}
\end{gathered}
$$

donde en $\Phi_{e}, \rho_{\text {relleno }}$ es $\rho_{s}$ que indica que se carga flexuralmente rellenando el espacio vacante con sedimentos y $\mathbf{H}(\mathrm{k})$ es la transformada de Fourier de $\mathrm{h}(\mathrm{x})$, la altura entre la paleobatimetría y la batimetría actual como se observa en la parte superior de la Figura 6.12.

\section{5) Calcular la anomalía asociada a la sedimentación}

Para calcular la anomalía de la sedimentación sumamos las contribuciones de las tres interfaces arriba descriptas. Las interfaces y sus contribuciones gravitacionales de un 

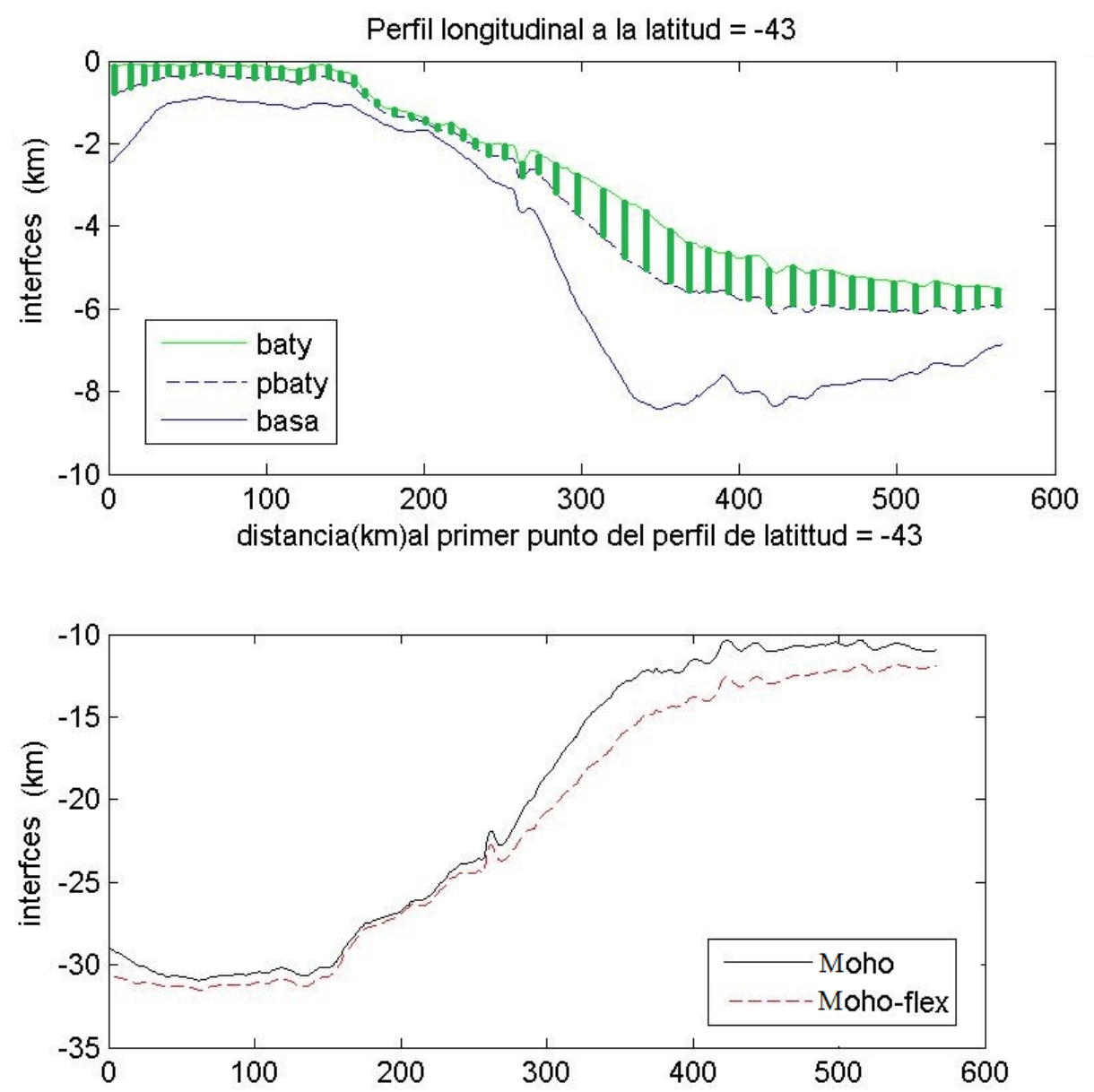

Figura 6.12. Carga de los sedimentos para el caso perfil $43^{\circ} \mathrm{S}$ y $T_{e}=10 \mathrm{~km}$, donde las líneas verticales verdes representan la altura de carga. baty: batimetría, pbaty: paleobatimetría y basa:basamento (arriba), moho-flex: Moho flexionado (abajo). Las líneas verdes verticales representan la altura de carga. 


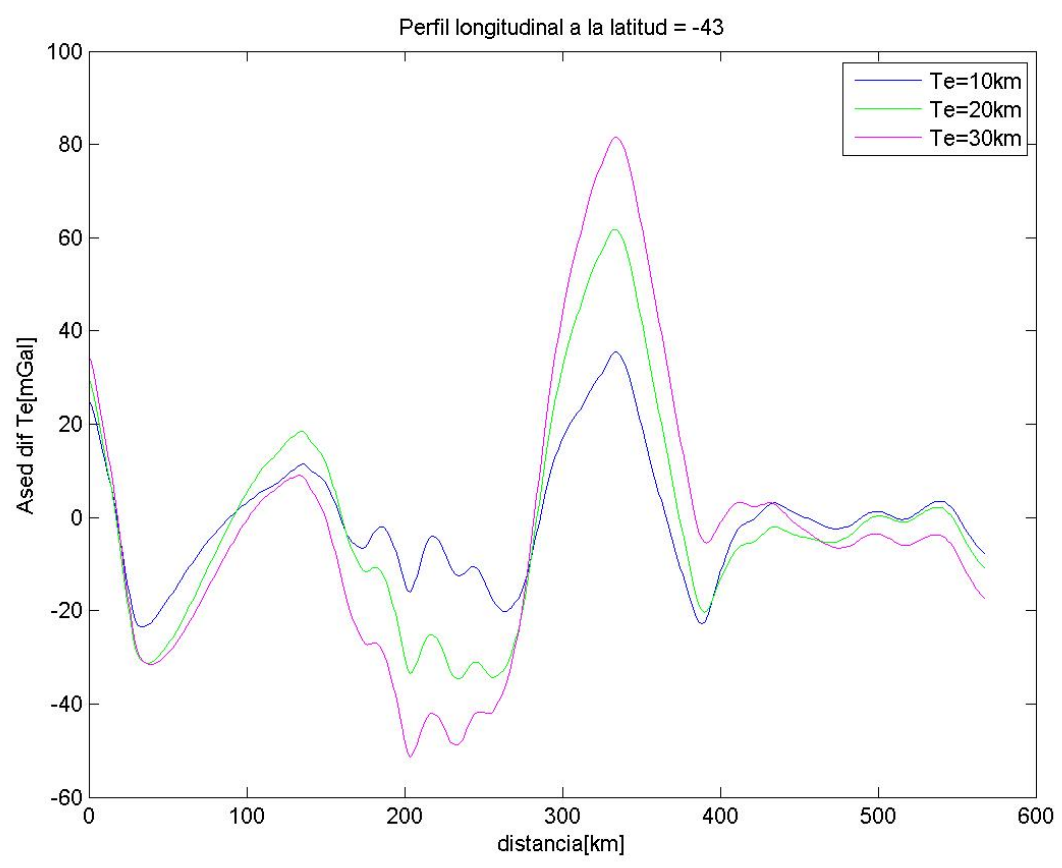

Figura 6.13. Anomalía de la sedimentación para el caso perfil $43^{\circ} \mathrm{S}$.

modelo sintético pueden visualizarse en la Figura 6.8. La forma ancha de la anomalía de la sedimentación está asociada con el alto central y dos lóbulos laterales. El "alto" es debido a que los sedimentos son más densos que el agua que desplaza la carga, mientras que el "bajo" es el resultado del desplazamiento de la relativamente baja densidad de la corteza dentro de un manto denso. En conjunto esto define la anomalía de la sedimentación (Fig. 6.13). Ésta depende fuertemente del $T_{e}$ de la litosfera. La carga de los sedimentos de un margen rígido $\left(T_{e}=35 \mathrm{~km}\right)([102]$ por ej. produce una anomalía de gran amplitud en el "alto" y "bajos" de menor amplitud. En contraste la misma carga cuando es depositada en un margen débil $\left(T_{e}=5 \mathrm{~km}\right)[102]$ resulta en dos altos sobre la forma de ancho original. El resultado neto de la anomalía de la sedimentación es modificar la anomalía del rift a través de una migración hacia el este del "alto" y los "bajos" respecto de la pendiente del talud original (la migración es más obvia para el caso de márgenes rígidos que débiles) [102].

\section{6) Obtener la anomalía suma, comparar con la anomalía observada y res- tringir el $T_{e}$}

Una vez estimadas la anomalía del rift y la de la sedimentación se genera la anomalía suma con la adición de ambas (Fig. 6.14). Dicha anomalía es una anomalía de aire libre estimada para el correspondiente $T_{e}$ que se comparará con la anomalía observada. La elección del $T_{e}$ vendrá dada por el mínimo del rms (error medio cuadrático) de la diferencia entre las anomalía suma y la observada.

En la Tabla 6.1 se muestra el resultado de la aplicación de la técnica POGM para perfiles longitudinales para distintos datos de espesor sedimentario: Secombi que describimos oportunamente en el Capítulo 5 y SedcombiCO, que es una compilación que incluye 


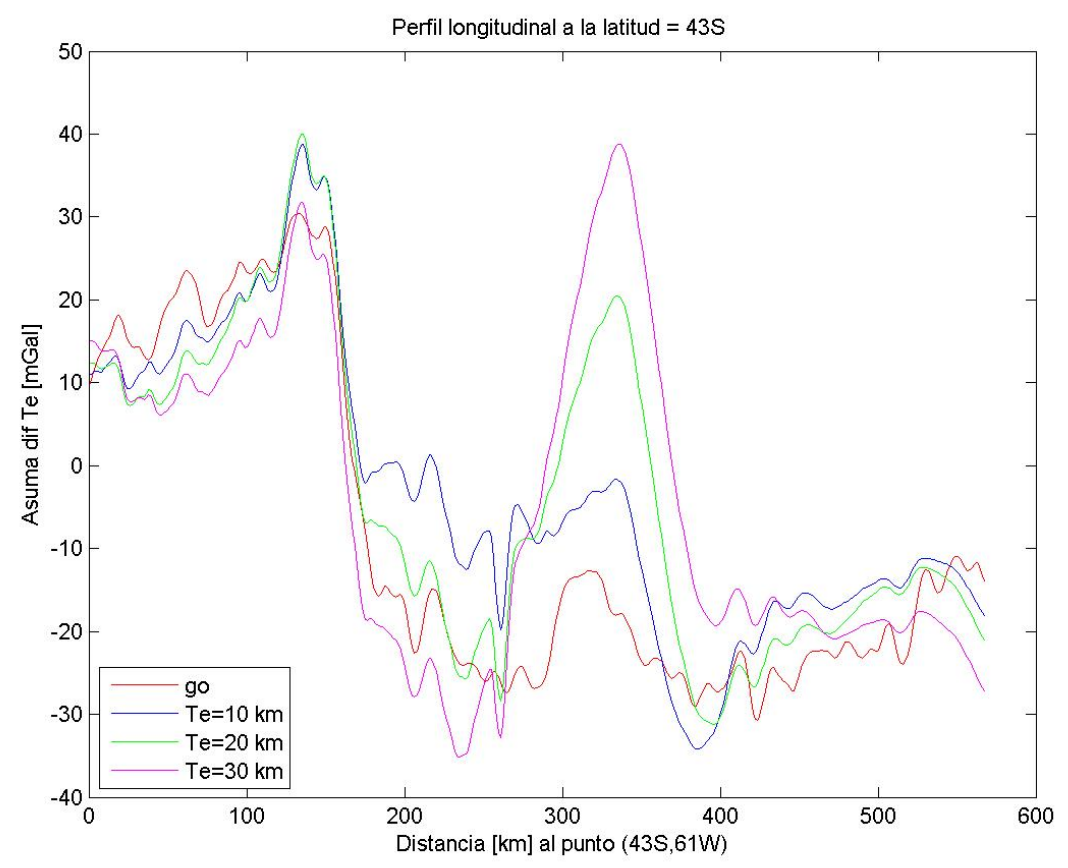

Figura 6.14. Anomalía suma para el caso perfil $43^{\circ} \mathrm{S}$.

datos de cruceros más modernos en la zona oceánica (COPLA) y datos de sedcombi en la zona de plataforma, una grilla en la zona oceánica fue cedida por representantes del Comité Nacional del Límite de la Plataforma Continental Argentina (COPLA) para este estudio y se generó una grilla combinada entre los datos de COPLA y la grilla Sedcombi que denominamos sedcombiCO. Los valores de rms de la Tabla 6.1 han sido seleccionados por el mínimo de rms para cada perfil para un $T_{e}$ variable de entre 0 y $30 \mathrm{~km}$. Notamos, que hay mayor variabilidad de los rms mínimos para el caso del espesor Sedcombi que para el de SedcombiCO. Pero al analizar los rms de cada perfil individualmente se ve que el rms mínimo difiere matemáticamente muy poco de los rms para otros $T_{e}$, por lo que no basta el criterio del rms mínimo si no que hay que hacer una inspección visual de la anomalía suma con la anomalía observada para ayudar a este criterio permitiéndo asignar un valor de $T_{e}$ adecuado. La tendencia general de disminución del $T_{e}$ hacia el norte estaría de acuerdo con el hecho de que la corteza oceánica es más joven dado que el margen se abrió de sur a norte [76].

En la Figura 6.15 se muestra la anomalía suma, resultado de la aplicación de la técnica POGM calculada para los datos de espesor sedimentario Sedcombi y SedcombiCO. Puede observarse en la Figura 6.15 al comparar el set de curvas con uno u otro dato la sensibilidad del cálculo al cambio del dato de espesor sedimentario, por lo que es deseable que este dato sea lo más fidedigno posible.

\subsubsection{Aplicación 3D}

Los pasos 1) al 6) pueden extenderse al caso en 3D. En primer lugar podemos aproximar el campo en 3D a través de agrupar perfiles 2D longitudinales separados por un espaciamiento latitudinal de 1'. Elegimos este espaciamiento para mantener la resolu- 


\begin{tabular}{ccccc}
\hline latitud & $T_{e}$ & rms & $T_{e}$ & rms \\
\hline-48 & 10 & 10.93 & 15 & 10.56 \\
-47 & 20 & 15.23 & 25 & 14.04 \\
-46 & 20 & 11.26 & 20 & 12.1 \\
-45 & 5 & 14.14 & 25 & 12.8 \\
-44 & 15 & 9.37 & 25 & 7.046 \\
-43.5 & 20 & 7.66 & 15 & 8.48 \\
-43 & 15 & 9.49 & 15 & 9.24 \\
-42 & 5 & 10.93 & 15 & 9.99 \\
-41 & 20 & 9.96 & 15 & 10.44 \\
-40 & 5 & 14.96 & 15 & 15.45 \\
-39 & 5 & 14.54 & 15 & 11.08 \\
\hline \hline grav 18.1 & \multicolumn{3}{c}{ sedcombi } & sedcombiCO
\end{tabular}

Tabla 6.1. rms del POGM para perfiles con diferentes valores de $T_{e}$ y a diferentes latitudes para dos datos de espesor sedimentario: Sedcombi y SedcombiCO

(a)

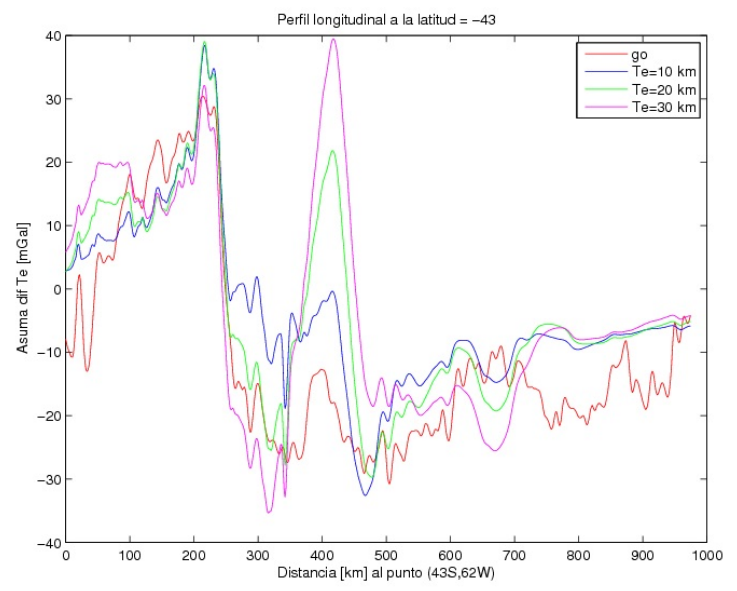

(b)

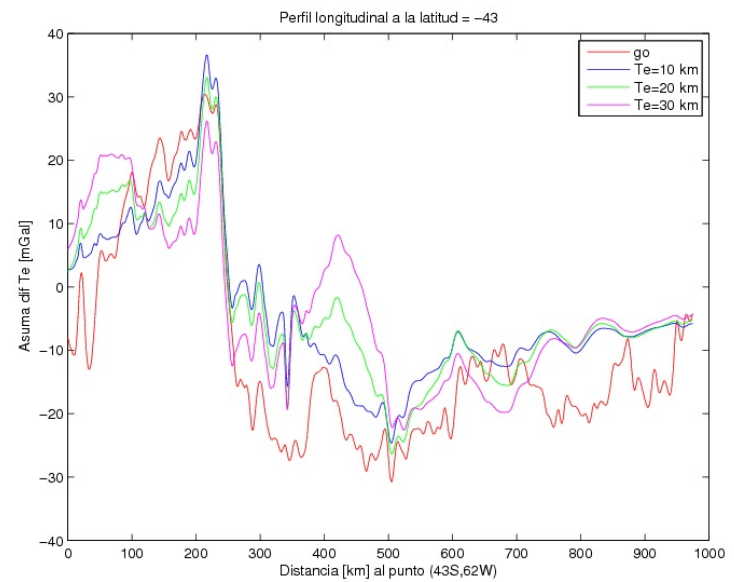

Figura 6.15. Anomalía suma para el caso perfil $43^{\circ} \mathrm{S}$ calculada para las grillas de espesor sedimentario Sedcombi (a) y SedcombiCO (b). 
(a)

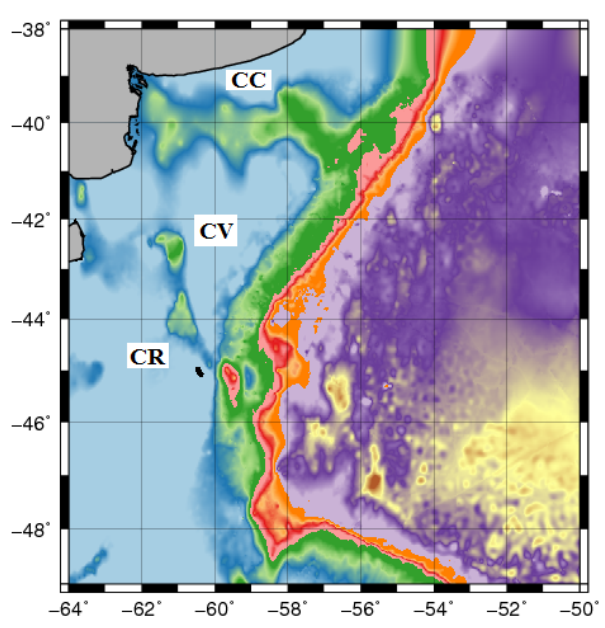

(b)

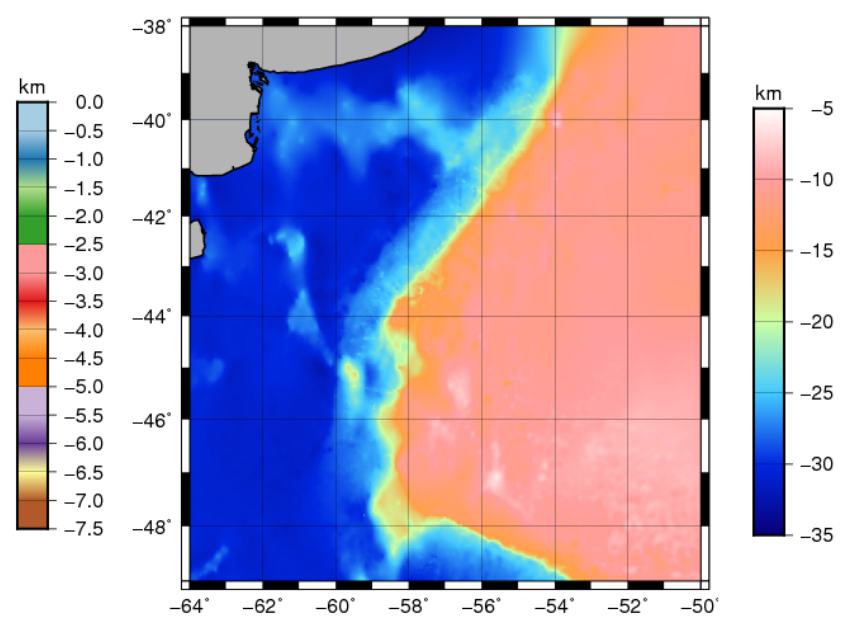

Figura 6.16. Una estimación $3 \mathrm{D}$ de la paleobatimetría al tiempo del rift (a) y del paleoMoho (b), a partir de perfiles 2D. CC: cuenca del Colorado, CV: cuenca de Valdés y CR: cuenca de Rawson.

ción espacial igual a la que tienen los datos observados. Luego podemos resolver todas las ecuaciones involucradas en los pasos 1) al 6) en 3D y comparar con la aproximación a partir de perfiles $2 \mathrm{D}$.

\section{1) Aplicar el backtripping a la carga de sedimentos - modo 3D}

En la Figura 6.16 se muestra en (a) el resultado de la técnica backstripping que nos permite visualizar una estimación de la paleobatimetría al tiempo del rift para las asunciones hechas y en (b) el paleo Moho. Pueden identificarse las cuencas del Colorado de Valdés y de Rawson y su compensación.

\section{2) Calcular el factor de estiramiento $\beta$, según Airy - modo 3D}

Un factor típico de valor de $\beta=2$ caracteriza cuencas sedimentarias intracontinentales. Un factor $\beta=5$ indica que puede producirse ruptura y la generación de nuevo océano [108].

Podemos decir que factores $\beta$ de hasta aproximadamente 5 representan en general corteza en extensión y existen en rifts en evolución. Para procesos de rift completos, con corteza oceánica y cuenca oceánica formadas, $\beta$ tiende a infinito. En nuestro caso la distribución areal de $\beta$ en la Fig. 6.17a muestra valores de $\beta$ cercanos a 2 en las cuencas del Colorado, Valdés y Rawson, mientras que la Fig. $6.17 \mathrm{~b}$ presenta valores de $\beta$ muy grandes en la zona oceánica profunda, especialmente en una región al sur de los $45^{\circ} \mathrm{S}$. Esto podría estar representando el hecho de que la extensión al crearse el océano fue aquí mucho mayor y más rápida, en concordancia con lo que sugieren los modelos tectónicos $[62,25,76]$.

Las cuencas de Colorado, Valdés y de Rawson aparecen en en los mapas de paleobatimetría al tiempo del rift y en el del factor de estiramiento $\beta$, en este último aparecen 
(a)

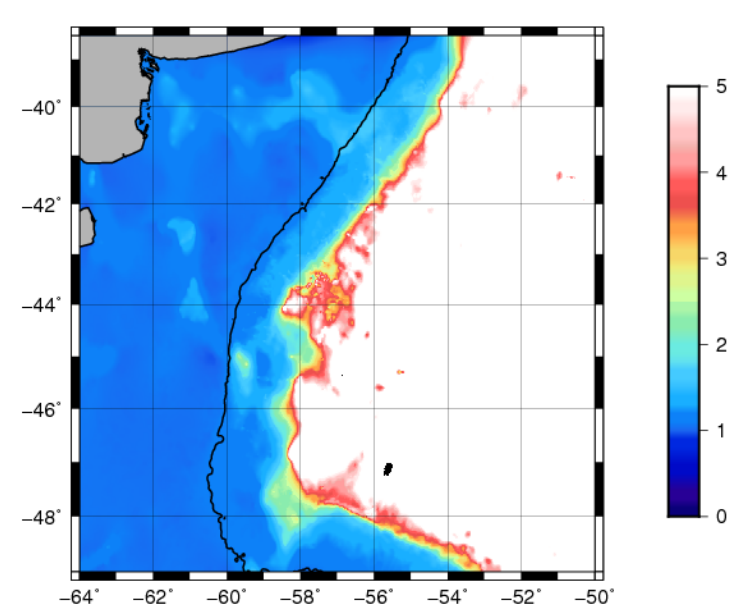

(b)

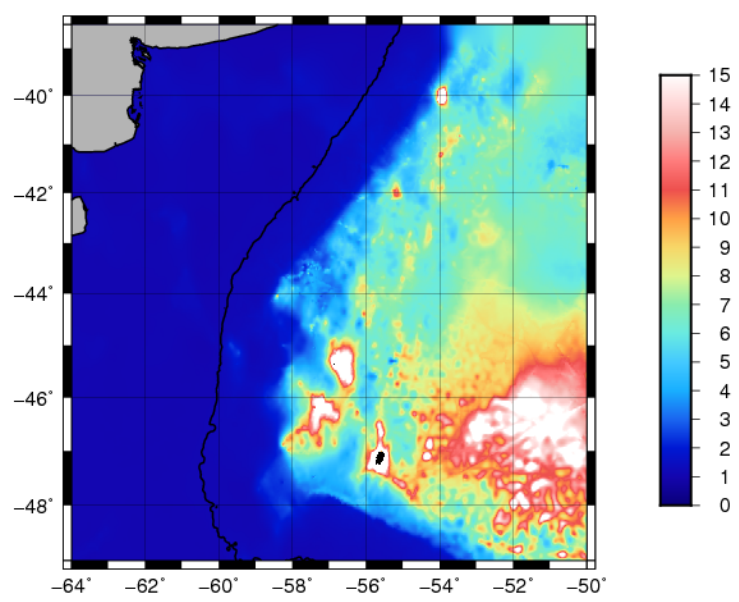

Figura 6.17. factor de estiramiento $\beta$ en a) enfatizando la zona de plataforma en b) la zona marina profunda. En ambas figuras se ha graficado en color negro la isobata de $500 \mathrm{~m}$ para referencia.

con un estiramiento mayor que el del entorno.

\section{3) Calcular la anomalía de gravedad asociada con el rift - modo 3D}

Lo primero que notamos es la diferente resolución entre la Figura 6.18a y 6.18b, donde la última no es simplemente un suavizado de la primera como se esperaría a priori por lo que pensamos que se trata de una cuestión numérica.

Analicemos más en detalle la Figura 6.18a: representa la anomalía de aire libre en el tiempo del rift (es decir, considerando a la sedimentación como un evento independiente, no simultáneo con el rift). Esto es una aproximación dado que hemos considerado a los sedimentos como una sola capa depositada luego del evento del rift. Se observa una alineación que comienza en las cuencas de Valdés y de Rawson y se continúa en una tercer posible cuenca lo que podría tratarse de un rift abortado o aulacógeno. Esto se evidencia en los mapas de paleobatimetría ( $6.16 \mathrm{a}$ ) y paleoMoho ( $6.16 \mathrm{~b}$ ) donde se puede observar que al estar el manto sustentando las cuencas esto da cuenta de un atenuamiento cortical. Puede pensarse en la unión de un punto triple entre el rift abortado y el rift que dio lugar al Atlántico.

\section{4) Restablecer la carga de los sedimentos, calcular la flexura del basamento y del Moho - modo 3D}

Se extiende a 3D las interfaces y se calcula la flexura del basamento que se aplica también al Moho. Puede observarse la flexura en la Figura 6.19 donde notamos una fuerte correlación con el espesor sedimentario (Fig. 5.3 del Capítulo 5), como cabía esperar. 
(a)

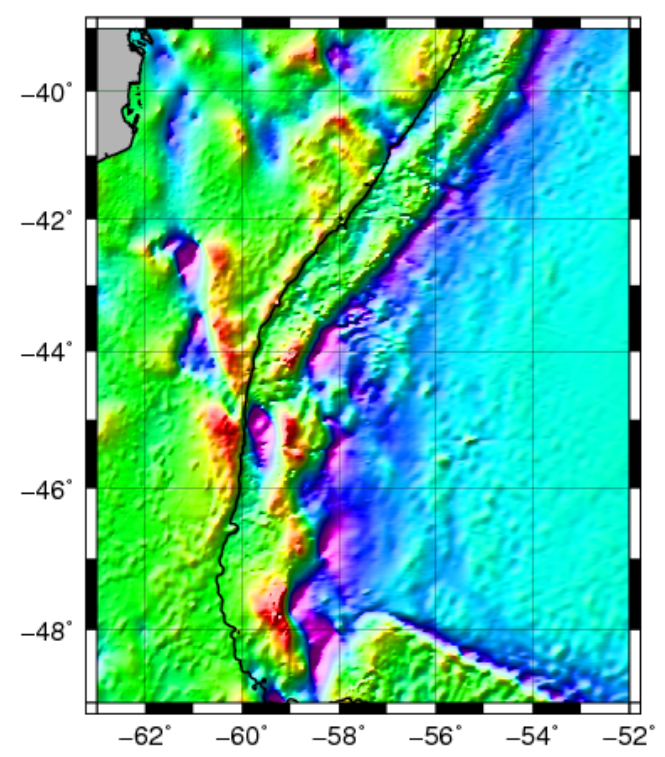

(b)

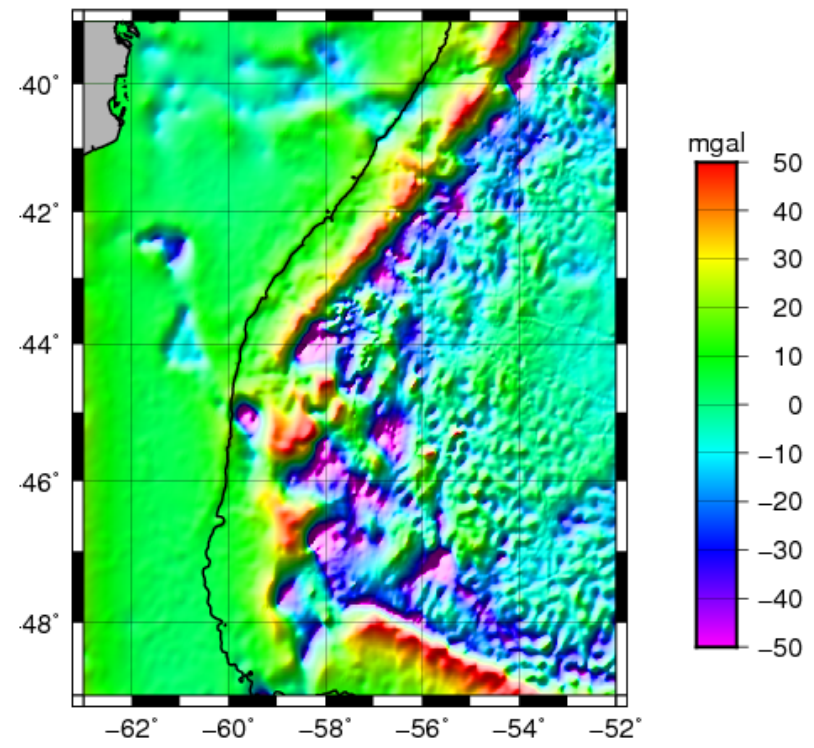

Figura 6.18. Anomalía del rift desde 2D (a) y desde 3D (b). En ambas figuras se ha graficado en color negro la isobata de $500 \mathrm{~m}$ para referencia.

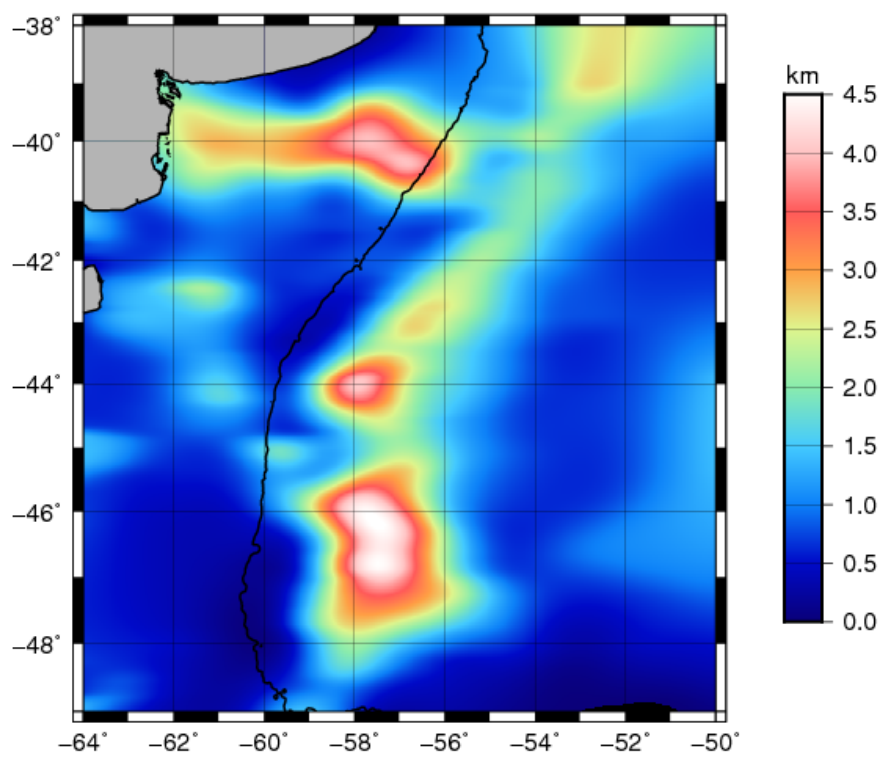

Figura 6.19. Flexura del basamento y del Moho. Se ha graficado en color negro la isobata de $500 \mathrm{~m}$ para referencia. 
(a)

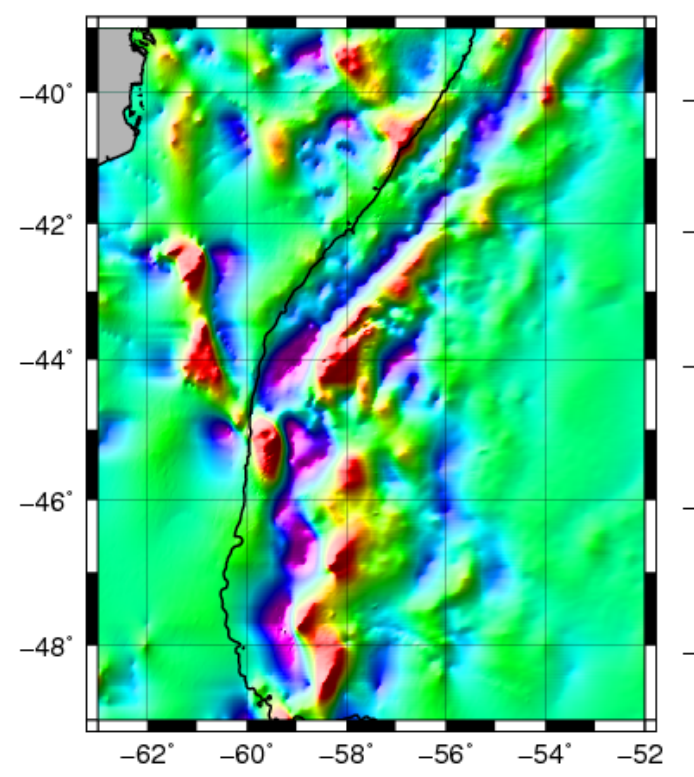

(b)

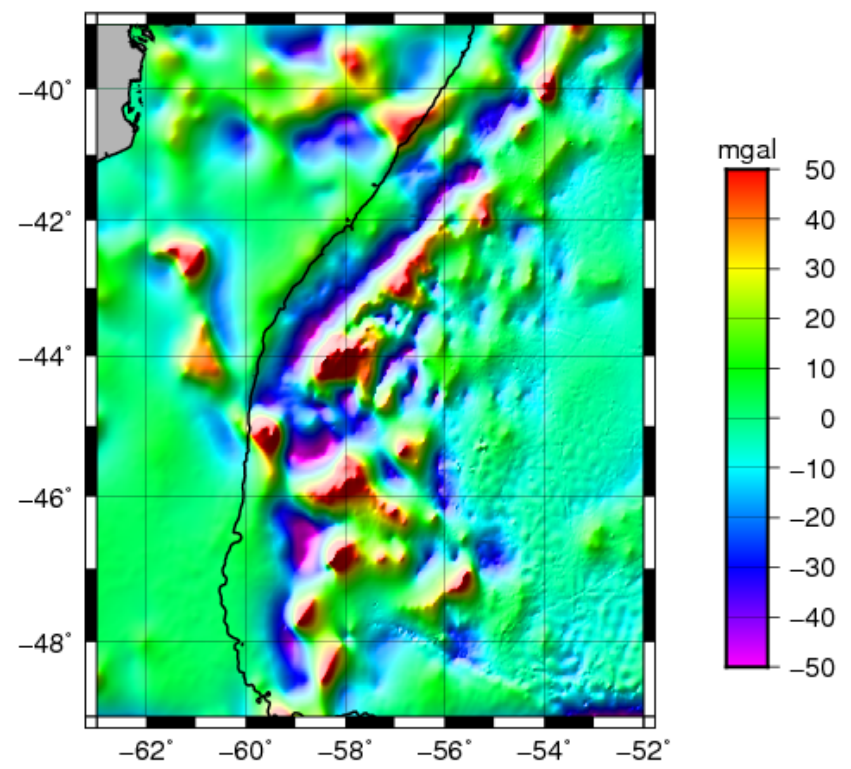

Figura 6.20. Anomalía de la sedimentación desde 2D (a) y desde 3D (b). En ambas figuras se ha graficado en color negro la isobata de $500 \mathrm{~m}$ para referencia.

\section{5) Calcular la anomalía asociada a la sedimentación - modo 3D}

Al ser el cálculo de la anomalía de la sedimentación un cálculo residual notamos que aquí la resolución se mantiene en ambas figuras 6.20a y 6.20b. Donde hay un máximo se traduce en un máximo de espesor sedimentario donde se ubica la carga. Donde hay mínimos son regiones de desplazamiento del manto y ubicación de la corteza en su reemplazo. En general se tiene un máximo acompañado de dos lóbulos laterales, esto representa una situación simétrica. Máximos con un mínimo más marcado que el otro hablarían de una situación asimétrica (asimetría de carga).

\section{6) Obtener la anomalía suma, comparar con la anomalía observada y res- tringir el $T_{e}$ - modo $3 \mathrm{D}$}

Notamos que la anomalía suma calculada con la aproximación 2D, Figura 6.21a, muestra el efecto de borde típico de los márgenes pasivos más marcado que el cálculo 3D (Figura 6.21b), y es la que más se asemeja al que presenta la anomalía observada Figura 3.1b del Capítulo 3, por lo que nuestra estimación 3D es mejor lograda a partir de la aproximación con perfiles 2D. Para restringir el $T_{e}$, elegimos aquel que minimiza la anomalía isostática. En la Figura 6.22a se representa el caso para $T_{e}=0 \mathrm{~km}$ (según Airy) y en la $6.22 \mathrm{~b}$ para $T_{e}=15 \mathrm{~km}$. Notamos, que $T_{e}=15 \mathrm{~km}$ es el que mejor minimiza la anomalía isostática (inspección visual) de entre varios valores de $T_{e}$ y el valor del rms también es mínimo como se observa en la Tabla 6.2.

La anomalía isostática calculada con el método flexural y minimizada para el caso $T_{e}=15 \mathrm{~km}$ además puede compararse con la obtenida con el método de Airy respecto del cual se observan rasgos suavizados. Se advierte que el método flexural es 
(a)

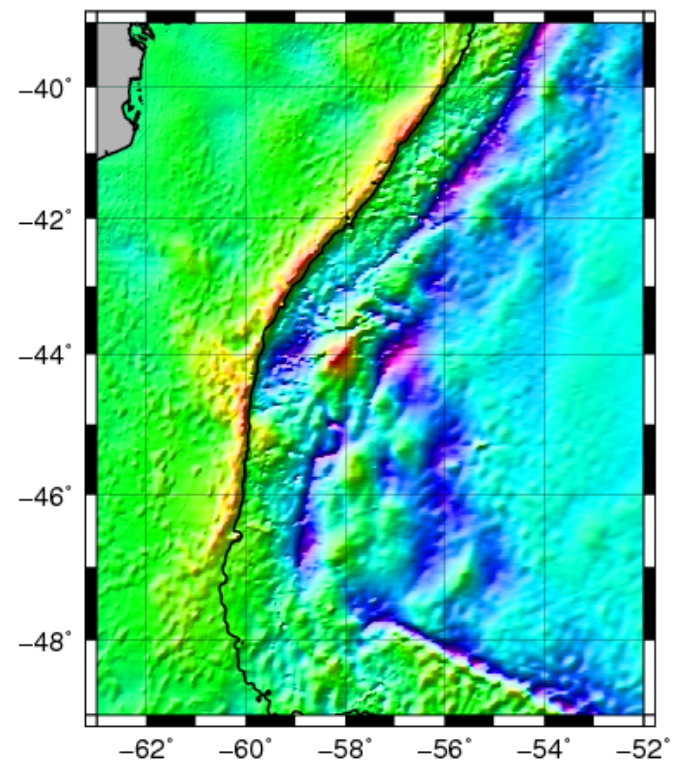

(b)

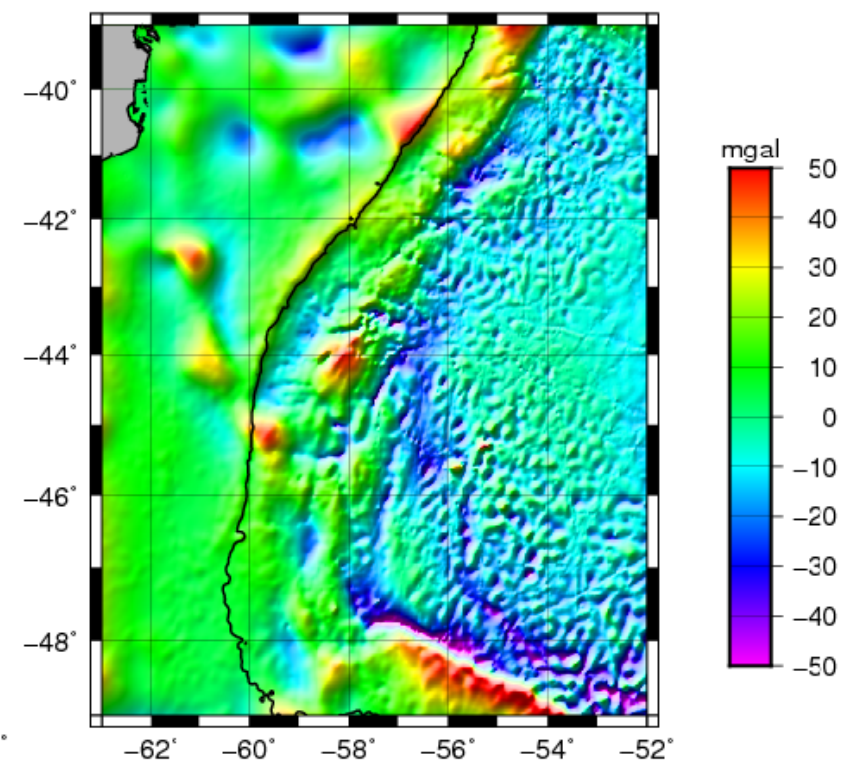

Figura 6.21. Anomalía suma desde 2D (a) y desde 3D (b). En ambas figuras se ha graficado en color negro la isobata de $500 \mathrm{~m}$ para referencia.

\begin{tabular}{ccc}
\hline Te & rms (3D) & rms (3D desde 2D) \\
\hline 0 & 17.13 & \\
5 & 16.94 & 12.43 \\
10 & 16.32 & 12.06 \\
15 & 15.89 & 11.85 \\
20 & 16.21 & 12.42 \\
25 & 17.31 & \\
\hline
\end{tabular}

Tabla 6.2. rms del POGM para grillas con diferentes valores de $T_{e}$ para las estimaciones: 3D y $3 \mathrm{D}$ a partir de 2D

sin dudas aquel con el que se obtiene la anomalía isostática mínima por lo que puede caracterizarse al margen con un espesor elástico de $T_{e}=15 \mathrm{~km}$.

\section{Relación con la segmentación y con las estructuras conocidas del margen}

En las Figuras 6.23 y 6.24 se muestran la anomalía del rift y la anomalía suma para el caso de la aproximación 2D. En la Figura 6.25 se muestran las anomalías isostáticas según Airy (Fig. 6.25a) y las calculadas con el POGM para un $T_{e}=15 \mathrm{~km}$ (Fig. 6.25b). En todas las figuras hemos superpuesto las estructuras digitalizadas de la segmentación del margen vista en el Capítulo 2.

En la Figura 6.23 es de notar que los grabens quedan encerrados entre el doble festón del efecto de borde la anomalía de aire libre e inmediatamente a la derecha del festón menos marcado quedan alineados paralelamente los SDRs. Además se observa que las características volcánicas del margen cambian al sur y al norte de la discontinuidad del 
(a)

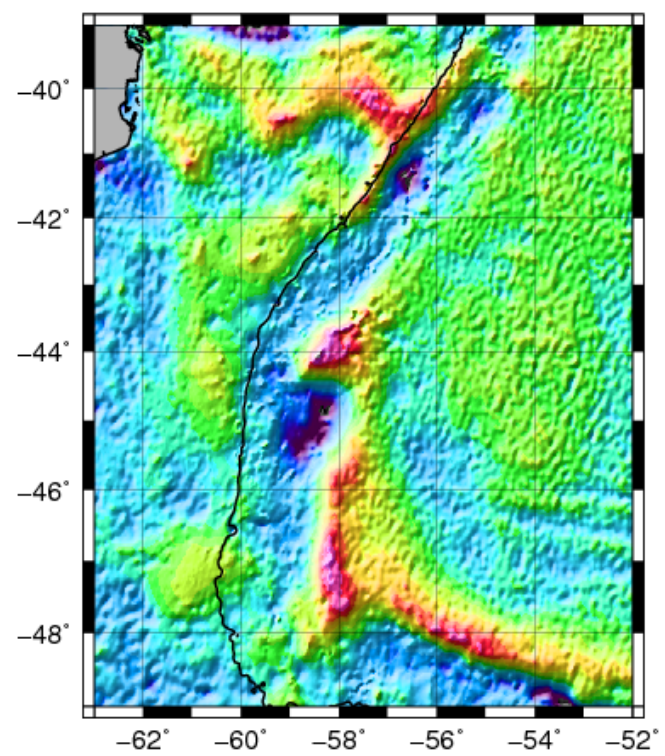

(b)

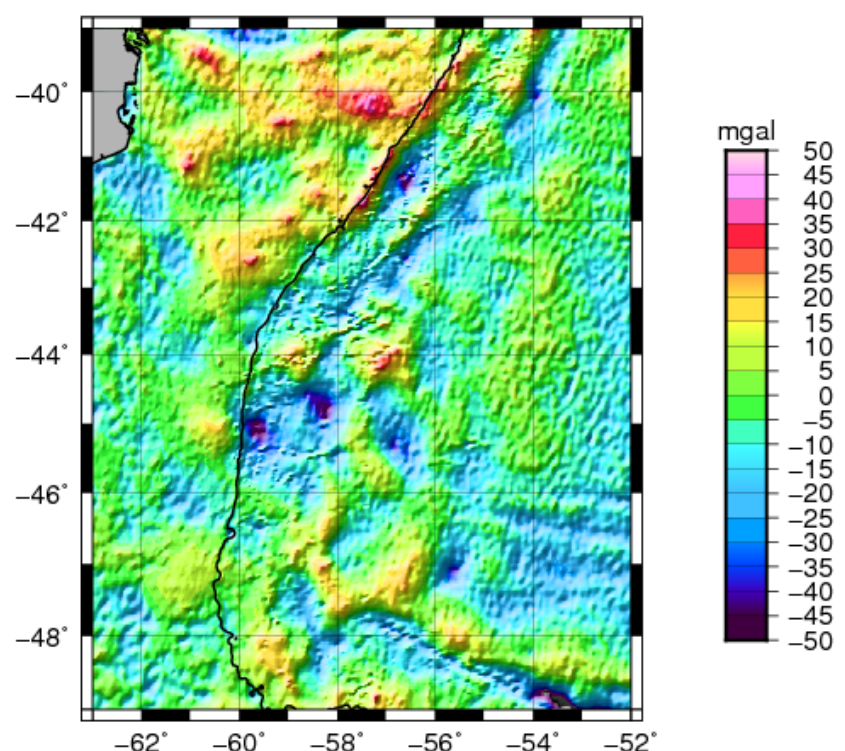

Figura 6.22. Anomalía isostática según Airy (a) y la calculada con POGM para $T_{e}=15 \mathrm{~km}(\mathrm{~b})$. En ambas figuras se ha graficado en color negro la isobata de $500 \mathrm{~m}$ para referencia.

Colorado o zona de transferencia del Colorado. En la Figura 6.24, también se observan los graben a derecha del efecto de borde de la anomalía de aire libre y luego las manifestaciones volcánicas alineadas con el cambio brusco de las anomalías de gravedad.

En la Figura 6.25a los graben quedan inmersos en una banda de valores negativos, dicha banda se atenúa un poco más en la Figura 6.25b. En la Figura 6.25a se observa un cambio brusco al norte y al sur de la discontinuidad del Colorado y una anomalía positiva en la zona de transferencia de Malvinas, en cambio en la Figura 6.25b estos rasgos se han atenuado.

En las Figuras 6.23 y 6.24 las cuñas volcánicas aparecen en zonas de depresiones gravimétricas por lo menos al norte de la discontinuidad del Colorado. Cabe aquí hacer notar que en nuestra aproximación no hemos calculado el efecto del subplacado magmático que puede existir asociado a los cuerpos de alta velocidad en general detectados por las líneas sísmicas debajo de los SDRs.

\subsection{Conclusiones del Capítulo 6}

Como resultado del análisis 2D se desprende que:

- El Te varía espacialmente pudiendo diferenciarse marcados sectores que podrían correlacionarse con la segmentación del margen.

- La anomalía suma sólo contiene los efectos de los procesos: rift y sedimentación. Puede adicionarse el efecto del magmatismo (subplacado magmático), para lo que se necesita conocer la disposición de los cuerpos de alta velocidad. 


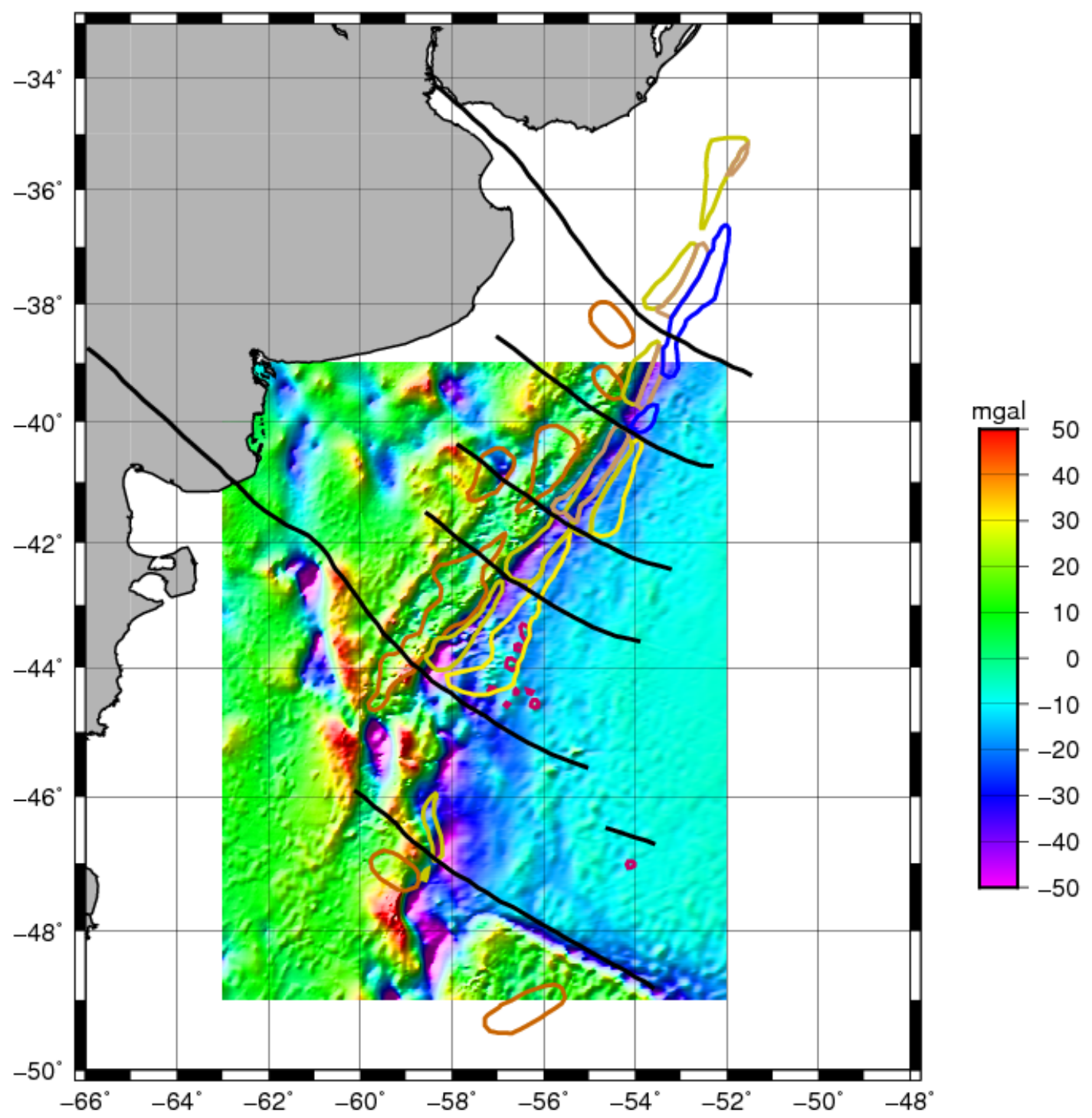

Figura 6.23. Anomalía del rift desde 2D, se han superpuesto las estructuras del mapa de segmentación del margen. Líneas marrón oscura representan pre-/syn rift grabens, líneas negras zonas de transferencia, líneas fucsia intrusivos, líneas verde claro son cuñas del mismo episodio 1, líneas marrón claro son cuñas del mismo episodio 2, líneas azul son depresiones del basamento oceánico y líneas amarillo son flujos de lavas basálticos. 


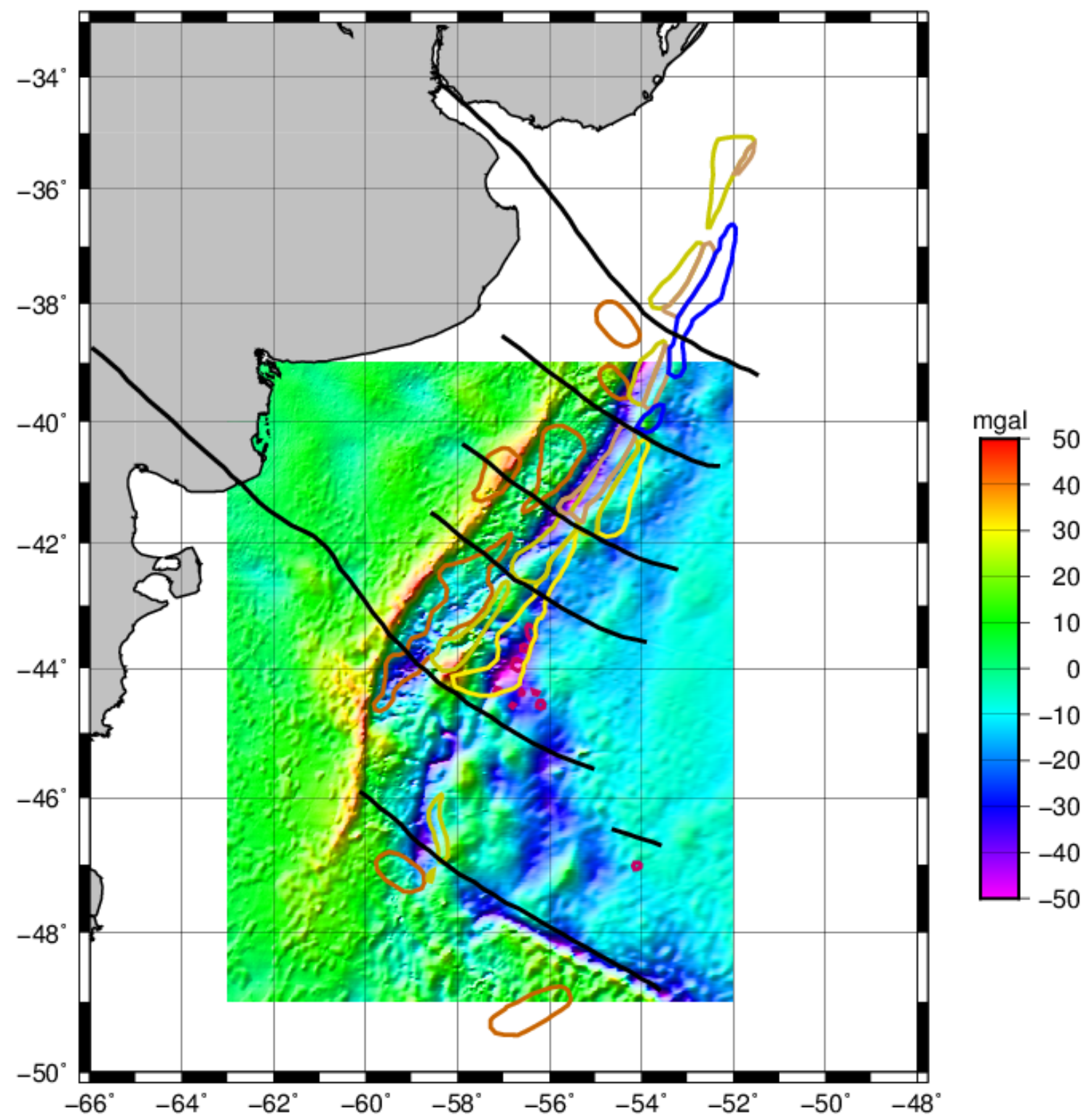

Figura 6.24. Anomalía suma desde 2D, se han superpuesto las estructuras del mapa de segmentación del margen. Líneas marrón oscura representan pre-/syn rift grabens, líneas negras zonas de transferencia, líneas fucsia intrusivos, líneas verde claro son cuñas del mismo episodio 1, líneas maroón claro son cuñas del mismo episodio 2, líneas azul son depresiones del basamento oceánico y líneas amarillo son flujos de lavas basálticos. 
(a)

(b)

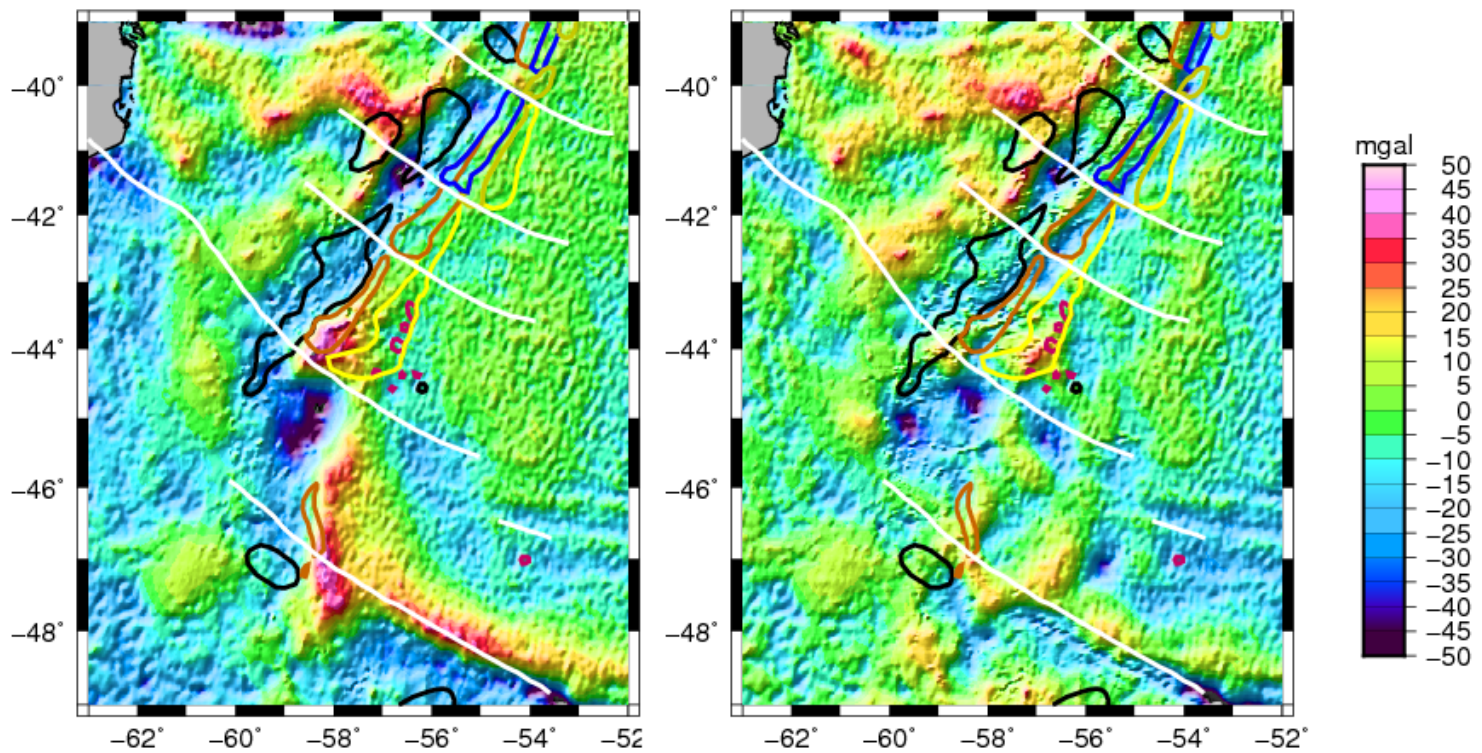

Figura 6.25. Anomalía isostática según Airy (a) y la calculada con POGM para $T_{e}=15 \mathrm{~km}$, se han superpuesto las estructuras de el mapa de segmentación del margen. Líneas negras representan pre-/syn rift grabens, líneas blancas zonas de transferencia, líneas fucsia intrusivos, líneas marrón oscuro son cuñas del mismo episodio 1, líneas azul son cuñas del mismo episodio 2, líneas verde claro son depresiones del basamento oceánico y líneas amarillo son flujos de lavas basálticos. 
- El POGM constituye la base necesaria para una interpretación que permite diferenciar la contribución que los diferentes procesos geológicos aportan a las anomalías de gravedad en zonas marinas.

Como resultado del análisis 3D surge que:

- La aproximación 3D a partir de 2D es comparable a la 3D e incluso tiene mayor resolución en la zona de plataforma, pero tiene una considerable lentitud en los cálculos.

- La alineación en la anomalía del rift de las cuencas de Valdés y de Rawson que se continúa en una posible tercer cuenca podría tratarse de un rift abortado o un aulacógeno. Esto puede comprobarse en los mapas paleobatimetría (Fig 6.16a) y paleoMoho (Fig 6.16b) como la evidencia de un atenuamiento cortical.

- La anomalía isostática muestra un fuerte residuo positivo en la zona de la cuenca del Colorado lo que podría indicar el hecho de que la cuenca se encuentre aun en subsidencia. 


\section{Capítulo 7}

\section{Conclusiones generales}

En esta tesis abordamos el desafío de la caracterización del sector volcánico del margen continental argentino a través del análisis de la isostasia. Para llevar a cabo este análisis, se necesitaron principalmente datos de gravimetría marina, batimetría y espesor sedimentario que adquirimos por convenios ya existentes, que son de dominio público o que fueron generados a partir de digitalizaciones de datos.

Sobre los datos de gravedad utilizados.

Analizamos datos de cruceros, creando una base de datos inicial para ser continuada en el futuro. Para el desarrollo de la tesis optamos por usar anomalías derivadas de altimetría satelital del modelo Sandwell \& Smith versión V18.1.

En un principio llevamos a cabo una evaluación previa de los datos disponibles, creando una base de datos de gravedad con datos de anomalía de gravedad marina medida a bordo de barcos, cuya primera etapa está completada parcialmente, como se explica en el texto de la tesis. Paralelamente comparamos grillas de anomalías de aire libre derivadas satelitalmente llegando a la conclusión de que los modelos DNSCO8GRA del DTU Space (National Space Institute) y la versión V18.1 de Sandwell \& Smith son los más adecuados para su utilización debido al uso de un mejor modelo geopotencial durante el procesamiento y principalmente, a los distintos procedimientos utilizados para su elaboración. Finalmente para los estudios que se hicieron a lo largo del desarrollo de la tesis se utilizaron las anomalías de aire libre derivadas de altimetría satelital de la versión V18.1 ya que poseen una amplia y total cobertura, además de que los resultados de la comparación realizada con datos de cruceros fueron más satisfactorios y que los modelos DNSO8GRA y DTU10, presentan un ruido latitudinal.

Resultados de estos análisis han sido publicados en un trabajo de una revista de circulación internacional [72] y se han expuesto en un congreso nacional en el año 2010.

Sobre los métodos de cálculo de las anomalías.

Experimentamos con diferentes métodos para calcular las anomalías de Bouguer, para poder luego, validar un método de cálculo de la anomalía isostática en zonas marinas. La anomalía de aire libre de un margen continental pasivo puede pensarse como la contribución de todas las masas y sus compensaciones en profundidad. Para calcularla, 
utilizamos un esquema de interfaces basado en el método de Parker. Este esquema puede incorporar las capas sedimentarias de manera mucho más simple que los métodos tradicionales y con mayor velocidad computacional, ya que buena parte de los cálculos puede desarrollarse en el dominio de los números de onda utilizando transformadas de Fourier. Para validar este método consideramos sólo las interfaces agua-corteza y corteza-manto y calculamos dos formas de obtener la anomalía de Bouguer. Una de ellas, considerando una adaptación del método de las interfaces y otra un método más tradicional que calcula la anomalía de Bouguer completa (Bullard A, B y C). Aunque la metodología de cálculo de la anomalía de Bouguer completa pueda considerarse robusta puede llegarse a un resultado similar con el esquema de las interfaces hasta orden 4. Las diferencias entre los métodos, el que calcula Bouguer completa y el que utiliza el desarrollo de Parker hasta orden 4, están altamente correlacionadas con la topografía. Observamos en perfiles que las mayores discrepancias entre los métodos se observan en las regiones donde el talud continental presenta variaciones locales de muy alta frecuencia, regiones donde el talud continental es muy pronunciado y las variaciones bruscas de pendiente no pueden ser bien representadas.

Resultados de estos análisis han sido publicados en una revista de circulación internacional [73]. Además se ha expuesto en un congreso nacional en el año 2012.

Una aproximación al cálculo de la anomalía isostática con el esquema de las interfaces se logra calculando la anomalía de aire libre con tres interfaces: agua-sedimento, sedimento-corteza y corteza-manto, y restándola a la anomalía de aire libre medida.

Sobre el tratamiento de la isostasia.

El análisis de la isostasia es fundamental para la interpretación gravimétrica del margen continental pasivo. Hemos comenzado analizando la anomalía isostática de Airy calculada con un esquema de tres interfaces y luego incorporamos el método flexural. Uno de los aspectos muy valiosos de esta tesis es que ha rescatado y valorado la originalidad que se encuentra en la aplicación de las técnicas de "backstripping", método que simula la descarga de los sedimentos conocidos, y en el Modelado de Gravedad Orientado por los Procesos (POGM) para evaluar la anomalía gravimétrica de aire libre en la zona de estudio utilizando todos los datos conocidos disponibles. De esta manera se obtiene una anomalía gravimétrica calculada que se sustrae de la anomalía de aire libre medida con más seguridad de que los rasgos residuales que persisten y que forman la anomalía isostática son en buen grado confiables como rasgos de masas no compensadas debido a fenómenos que es muy importante analizar.

Calculamos la anomalía isostática con el esquema de tres interfaces con compensación de Airy. Notamos que los resultados son fuertemente dependientes de los datos de espesor sedimentario, por lo que es deseable que dichos datos sean lo más fidedignos posible. En esta tesis trabajamos con datos de espesor sedimentario que provinieron de la contribución de tres fuentes obtenidas de mapas publicados y con otros datos contenidos en una grilla con información de cruceros.

Resultados de estos análisis se han enviado para su publicación en una revista de circulación internacional [74]. Además se ha expuesto en un congreso nacional en el año 2012.

Con posterioridad, se utilizó el concepto de isostasia flexural en la que se considera 
a la topografía como una carga que causa un combamiento de la litosfera que puede modelarse bajo la teoría de flexión de placas. Si consideramos a la litosfera con una cierta rigidez constante y que bajo las aproximaciones de placa delgada responde a las cargas con un comportamiento flexural, la litósfera se comporta como un filtro pasabajos entre la carga y la deformación flexural asociada, siendo el corte en frecuencias determinado por la rigidez de la placa (y por ende por su $T_{e}$,).

Con la técnica backstripping podemos estimar la profundidad del basamento en ausencia de sedimentos, es decir la paleobatimetría en el tiempo del rift. Para el caso de backstripping flexural se determina un parámetro apropiado en el espacio de los números de onda, que modifica la respuesta de Airy produciendo la respuesta flexural.

El POGM utiliza información sísmica del espesor sedimentario y del volcanismo presente (reflectores sísmicos que inclinan hacia el mar (SRDs), subplacado, etc.) de la configuración del margen actual, para estimar la anomalía de gravedad que está asociada a los procesos: rifting, sedimentación y magmatismo. El objetivo del POGM es discriminar la contribución que los diferentes procesos geológicos aportan a la anomalía observada y el del backstripping es determinar la geometría del margen al tiempo del rift.

Hemos implementado el método flexural para la aplicación de las técnicas mencionadas, de manera que puede disponerse de las respuestas gravimétricas de Airy (hidrostática) y flexural, que contempla las propiedades elásticas de la litosfera terrestre. Por ahora calculamos el backstripping removiendo los sedimentos en una sola etapa, pero tenemos presentes las limitaciones que esto implica. Asimismo, tampoco hemos aún incorporado el cálculo del subplacado magmático. Merece hacerse notar que los cálculos para estas técnicas son complejos y consideramos que aun con estas limitaciones hemos logrado un importante avance.

Los resultados de los análisis del POGM se han expuesto en dos congresos nacionales en el año 2014 y se planea hacer una publicación en una revista de circulación internacional.

Sobre los resultados de la anomalía isostática y el POGM.

Analizamos los resultados del cálculo de la anomalía isostática según Airy y de la anomalía isostática derivada luego de la aplicación de la técnica POGM.

La anomalía isostática según Airy presenta una zona de valores negativos en la zona del talud en algunos lugares a veces muy intensos. Es en esta zona donde el análisis de datos sísmicos descriptos en el texto muestra la presencia de grabens en el basamento. En la cuenca del Colorado aparecen anomalías positivas que pueden indicar que la cuenca estaría en subsidencia. Las cuencas de Valdés y de Rawson se ven como señales positivas débiles. Justo en la zona donde las características del margen cambian aparecen un par de anomalías, una de ellas de forma casi circular negativa y la otra triangular positiva aproximadamente en la zona de la discontinuidad del Colorado. Comparando con el mapa de espesor sedimentario la zona positiva coincide con un máximo (cuenca Argentina), que por ahora tentativamente podría interpretarse como una cuenca en subsidencia, pero merece realizarse un análisis más exhaustivo. Podría también ocurrir que en ese lugar hubiera un alto en el basamento o también podría tratarse de una intrusión en profundidad de material más denso o que la cuenca por 
su tamaño pequeño esté en equilibrio isostático según el modelo flexural.

Aplicamos la técnica POGM a perfiles en los que basándonos en el criterio del rms mínimo (entre la anomalía suma y la anomalía observada o medida) encontramos diferentes valores de $T_{e}$ a lo largo del margen. Este análisis se ha hecho hasta ahora con perfiles longitudinales. Ya que del análisis de cada perfil se obtiene un valor de $T_{e}$, reconocemos la conveniencia de analizar perfiles aproximadamente perpendiculares al margen para ver si estas variaciones pueden correlacionarse con la segmentación del margen, pero lo haremos con posterioridad.

Utilizando el resultado de aplicar la técnica a perfiles equiespaciados muy próximos podemos reconstruir la información en 3 dimensiones y luego compararla con la que resulta de resolver las ecuaciones en 3 dimensiones directamente. Al hacer esta comparación hemos encontrado que la anomalía suma calculada con la aproximación 3D a partir de perfiles 2D arroja mejores resultados, siendo una buena manera de representar ésta técnica en 3D. La anomalía de aire libre calculada (anomalía suma) puede ser discretizada según sea el proceso involucrado, pero en esta tesis hemos calculado la anomalía del rift y la anomalía de la sedimentación como componentes de la anomalía suma. Como rasgos a destacar en la anomalía del rift puede observarse una alineación entre las cuencas de Valdés, Rawson y una posible tercer cuenca. Dicha alineación podría tratarse de un aulacógeno o rift abortado. Al comparar la anomalía suma con la anomalía de aire libre observada se obtiene una anomalía isostática que resulta ser mínima según inspección visual y criterio de rms para un $T_{e}=15 \mathrm{~km}$. Si esta anomalía se compara con la obtenida según Airy se observa que la derivada a partir de las técnica POGM es mínima y que en ella se puede reconocer como rasgo importante que aun persiste un fuerte residuo positivo en la zona de la cuenca del Colorado que podría estar de acuerdo con la idea de que la cuenca se encuentre aun en subsidencia según fuera indicado por Introcaso et al. (1984) con anterioridad [48].

Los efectos de las anomalías circular y triangular que se observaban en la anomalía isostática de Airy se han atenuado en gran medida con el nuevo método, lo cual indicaría la eficacia del método flexural .

\section{Sobre los cálculos.}

La implementación de estos métodos se ha hecho casi totalmente con programas de cálculo propios, desarrollados en el transcurso de la tesis. La excepción han sido rutinas del paquete de programas GMT y dos programas publicados que se usaron básicamente para análisis de métodos (FA2BOUG, 3Dinv).

\section{Sobre el método gravimétrico.}

Nuestros modelos se basan en los métodos potenciales, por lo que hay una ambigüedad inherente en los resultados. Creemos que al considerar la técnica combinada de backstripping y el Modelado de Gravedad Orientado por los Procesos, se reduce esta ambigüedad principalmente por tener en cuenta la manera en que los sedimentos flexionan la corteza.

En recientes publicaciones (Zalán et al. [110, 111]), se discute la interpretación de secciones de sísmica 2D ultra profundas que permiten tener una imagen de los márgenes 
con mucho detalle que posibilita identificar los SDRs, el subplacado magmático y otros aspectos. El tratamiento que hemos hecho de los datos de gravedad en esta tesis no contiene todo el detalle que puede obtenerse a partir de esta sísmica adquirida con tecnología muy moderna. Los datos de sísmica de esta naturaleza no están disponibles, y además sería demasiado costoso que pudiera con ella cubrirse parte o toda la extensión del margen continental argentino. El grado de complejidad y detalle de esta sísmica no puede lograrse con datos de gravedad pero éstos tienen a su favor la ventaja de que son de público y libre acceso y que pueden analizarse en una gran área, como hemos hecho a lo largo de toda la Tesis. 



\section{Apéndice A}

\section{A.1 Flexura de Placas en dos dimensiones}

Según Turcotte y Schubert [89] la tectónica de placas implica que las rocas cerca de la superficie son rígidas y por lo tanto tienen un comportamiento elástico a escala de tiempo geológico. Las delgadas placas de la superficie constituyen la litosfera que flota en un manto relativamente dúctil. Las placas están sujetas a una variedad de cargas como por ejemplo debidas a los volcanes o montañas marinas que fuerzan a la litosfera a combarse bajo su peso. El relativo combamiento o flexura de la litosfera observada nos permite conocer las cargas en superficie, con lo que podemos deducir las propiedades elásticas de las placas y su espesor. Lo primero que tenemos que desarrollar es la teoría de la flexura de placas en respuesta a las fuerzas y torques aplicados. Hay muchas aplicaciones geológicas que pueden ser abordadas con esta teoría que pueden ayudarnos a entender por ej.: sistemas de plegamientos en cadenas montañosas y modelarlos como deformaciones de placas elásticas sujetas a fuerzas compresivas horizontales o modelar levantamientos de estratos sobre intrusiones ígneas, entre otros[89].

Considerando una placa delgada de material elástico. Si la placa es lo suficientemente delgada, podemos aproximar las fuerzas actuantes en y dentro de ella como sigue: Sea $w(x)$ el desplazamiento de la placa desde la horizontal. Las fuerzas y momentos actuando en un pequeño segmento de la placa, como se ilustra en la Figura ( A.1), donde $q$ es la fuerza por unidad de longitud actuando en la placa, $V$ es la fuerza de cizalla actuando en los bordes de la porción de placa (esfuerzo de cizalla integrado en el borde), $P$ es la presión en los bordes (esfuerzo normal integrado; debe estar en equilibrio debido a que no hay movimientos de la placa de izquierda o derecha), $M$ es el momento actuando en el borde de la porción de placa (momento del esfuerzo normal). Si se plantea el equilibrio de las fuerzas en la dirección vertical, se tiene:

$$
\begin{gathered}
q(x) d x+d V=0 \\
\frac{d V}{d x}=-q
\end{gathered}
$$

El equilibrio de las fuerzas en la dirección horizontal $(x)$ se da debido a que $P$ es constante. Sumando los momentos actuantes en el segmento de la placa encontramos:

$$
\begin{gathered}
d M-P d w=V d x \\
\frac{d M}{d x}=V+P \frac{d w}{d x}
\end{gathered}
$$




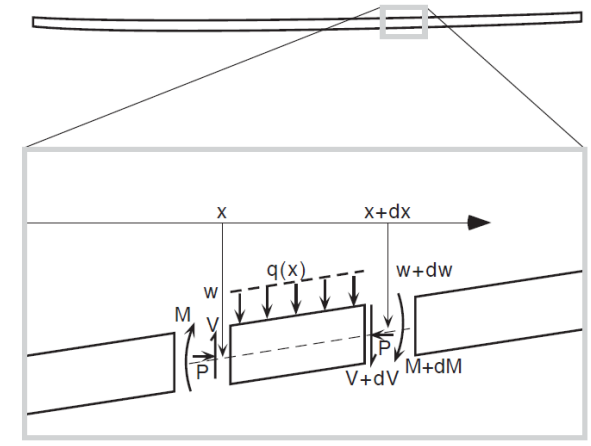

Figura A.1. Fuerzas y torques en una sección pequeña de una placa flexionada, http://www.colorado.edu/geolsci/courses/GEOL5690/FlexureNotes.pdf (Turcotte \& Schubert) [89].

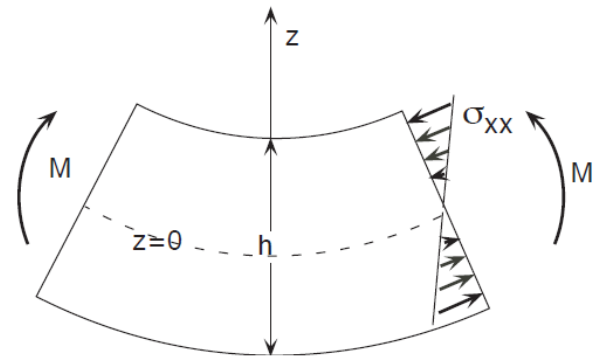

Figura A.2. Esfuerzo normal en una sección transversal de una placa elástica curvada (Turcotte \& Schubert) [89].

Podemos eliminar $V$ combinando las ecuaciones A.1 y A.2 para obtener:

$$
\frac{d^{2} M}{d x^{2}}=-q+P \frac{d^{2} w}{d x^{2}}
$$

Si analizamos a $M$ más en detalle, encontramos que esta relacionado con $z$ como se observa en la Figura A.2.

En primer lugar consideremos el esfuerzo actuante en el borde de la placa (estos son esfuerzos desviatorios; hemos substraído el esfuerzo medio $P / h$ ). El momento total resulta:

$$
M=\int_{\frac{-h}{2}}^{\frac{h}{2}} \sigma_{x x} z d z
$$

Considerando la placa en su estado original y comparando con el de la Figura A.2 podemos ver que existe un acortamiento en la parte superior y un estiramiento en la parte inferior. La línea de puntos representa una superficie neutral, la cual se encuentra no deformada por la flexión. A partir de la ley de Hook que relaciona linealmente esfuerzos y deformaciones para un medio elástico e isótropo se tiene:

$$
\begin{aligned}
& \varepsilon_{x x}=\frac{1}{E}\left(\sigma_{x x}-\nu \sigma_{y y}\right) \\
& \varepsilon_{y y}=\frac{1}{E}\left(\sigma_{y y}-\nu \sigma_{x x}\right)
\end{aligned}
$$




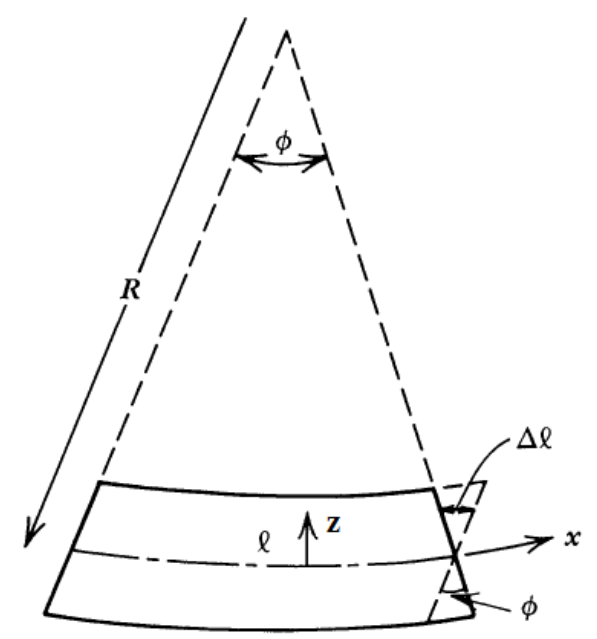

Figura A.3. Extensión y contracción longitudinal a una distancia $z$ desde la mitad del plano de la placa (Turcotte \& Schubert) [89].

Donde $E$ es el módulo de Young (del orden de $70 \mathrm{GPa}$ para la corteza), y $\nu$ es el radio de Poisson (del orden de 0.25 para la corteza) e $y$ es perpendicular a $x$ en el plano horizontal, esto es, $\varepsilon_{y y}=0$. Podemos combinar éstas ecuaciones para obtener:

$$
\sigma_{x x}=\frac{E}{\left(1-\nu^{2}\right)} \varepsilon_{x x}
$$

ésta puede reemplazarse en la ec. A.4 para dar:

$$
M=\frac{E}{\left(1-\nu^{2}\right)} \int_{\frac{-h}{2}}^{\frac{h}{2}} \varepsilon_{x x} z d z
$$

Las deformaciones están geométricamente relacionadas con el radio de curvatura de la placa, $R$, asumiendo un radio de curvatura extremadamente largo:

$$
\varepsilon_{x x}=-\frac{\Delta l}{l}=\frac{z}{R}
$$

Con ayuda de la geometría (Fig. A.4) podemos encontrar que el ángulo $\phi$ subtendido por la longitud $l$ (aproximadamente $d x$ ) es:

$$
\phi=d \alpha=\frac{d \alpha}{d x} d x=\frac{d}{d x}\left(-\frac{d w}{d x}\right) d x=-\frac{d^{2} w}{d x^{2}} d x,
$$

siendo $\phi=l / R$ podemos encontrar que:

$$
\frac{1}{R}=\frac{\phi}{l} \approx \frac{\phi}{d x}=-\frac{d^{2} w}{d x^{2}}
$$

Haciendo la substitución en la ec. ( A.8) se tiene:

$$
\varepsilon_{x x}=-z \frac{d^{2} w}{d x^{2}}
$$




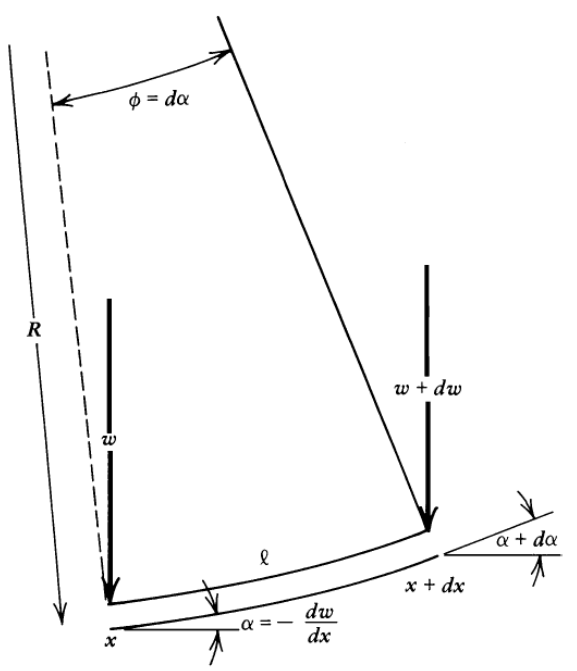

Figura A.4. Esquema ilustrativo de la relación geométrica de la placa flexionada (Turcotte \& Schubert) [89].

introduciendo ésta en la ecuación A.7, el momento flexural puede escribirse como:

$$
\begin{aligned}
M & =\frac{-E}{\left(1-\nu^{2}\right)} \frac{d^{2} w}{d x^{2}} \int_{\frac{-h}{2}}^{\frac{h}{2}} z^{2} d z \\
= & \frac{-E}{\left(1-\nu^{2}\right)} \frac{d^{2} w}{d x^{2}}\left(\frac{z^{3}}{3}\right)_{-\frac{h}{2}}^{\frac{h}{2}} \\
& =\frac{-E h^{3}}{12\left(1-\nu^{2}\right)} \frac{d^{2} w}{d x^{2}} .
\end{aligned}
$$

El coeficiente de $-\frac{d^{2} w}{d x^{2}}$ en el lado derecho de la ecuación es llamado rigidez flexural $D$ de la placa, reemplazando $h$ por $T_{e}$ (espesor elástico de la placa) se obtiene una expresión que relaciona la rigidez flexural con los parámetros elásticos

$$
D \equiv \frac{E T_{e}^{3}}{12\left(1-\nu^{2}\right)}
$$

Reemplazando esta última en la ecuación A.3

$$
D \frac{d^{4} w}{d x^{4}}=q(x)-P \frac{d^{2} w}{d x^{2}} .
$$

Esta ecuación para la deflexión de una placa puede resolverse para un número simple de casos y aplicar los resultados a la deformación de estratos y flexura de la litosfera. En el contexto de una placa elástica sobre un manto dúctil, esta ecuación debe ser modificada teniendo en cuenta una fuerza restitutiva (empuje) que actúa hacia arriba en la placa deflectada.

Cuando una carga aplicada flexiona la placa, la región deflectada es ocupada con agua, como en el caso de litosfera oceánica o por una cuenca continental sin sedimentos o con sedimentos, como la mayoría de las cuencas adyacentes interiores sometidas a erosión. El material de relleno tiene una densidad menor que la del manto, el cual está siendo 
reemplazado. La magnitud de la fuerza restauradora en la base de la placa deflectada puede estimarse considerando el balance de presión $(\rho g h)$ bajo la región de máxima deflexión y bajo la región que no se deflecta. Esta fuerza restauradora es $\rho g w$ y la fuerza vertical neta actuante resulta de la diferencia entre la carga aplicada y la fuerza hidrostática restitutiva.

La ecuación A.14 puede expresarse como:

$$
D \frac{d^{4} w}{d x^{4}}+P \frac{d^{2} w}{d x^{2}}+\Delta \rho g w=q_{a}(x) .
$$

donde $\Delta \rho$ es $\left(\rho_{m}-\rho_{w}\right)$ para una cuenca solo rellenada con agua y $\Delta \rho$ es $\left(\rho_{m}-\rho_{s}\right)$ para una cuenca totalmente rellena de sedimentos. La litósfera tiene una respuesta flexural de acuerdo a la distribución espacial de la carga.

Si la longitud de onda de la carga es suficientemente corta, la deflexión vertical de la litosfera es pequeña y la litosfera puede considerarse con rigidez infinita para la carga a éstas escalas. Si la longitud de onda de la carga es suficientemente grande hay una respuesta isostática efectiva tendiente a alcanzar el equilibrio hidrostático y la litosfera parece no tener rigidez. El grado de compensación de la carga topográfica es el radio de la deflexión de la litosfera que tiene un máximo o deflexión hidrostática. 



\section{Apéndice B}

\section{B.1 Cálculo de anomalías de gravedad 2D}

Estructuras geológicas que están alargadas en una dimensión, como zonas de fracturas, fallas, dikes, zonas de rift, anticlinales están a veces alineados en una dirección horizontal y las anomalías de gravedad y magnéticas que generan están también alineadas en una dirección. Si las anomalías son lo suficientemente "lineales' 'es posible considerar las fuentes gravitacionales y magnéticas como completamente invariantes en la dirección paralela a la dirección elongada. Si el eje es paralelo a la dirección de invarianza, el cuerpo se dice que es bidimencional o 2D. Un cuerpo que sea 20 veces mas largo que ancho puede considerarse en 2D [9].

Las fuentes 2D son mas faciles de conceptualizar y consideradas más fáciles de modelar que los 3D, lo cual es una ventaja cuando las situaciones geológicas lo permiten. Un camino útil de aproximar situaciones geológicas es reemplazar la forma de la sección transversal del cuerpo por polígonos simples.

El método aquí descripto sigue el paper de Hubbert [47] pero Talwani, Worzel y Landisman [87] son los primeros en presentar el método con un algoritmo adaptado para computadoras.

La siguiente derivación provee el mismo resultado que Talwani et al. [87] por un camino diferente [9]. El potencial gravitacional de un cuerpo en dos dimensiones con una densidad volumétrica $\rho(x, z)$ puede escribirse como:

$$
U=2 G \int_{S} \rho(S) \log \frac{1}{r} d S
$$

donde la integración es sobre una superficie de sección transversal S y donde $\mathrm{r}$ es la distancia entre el punto de calculo y un elemento del cuerpo dado por:

$$
r=\sqrt{\left(x-x^{\prime}\right)^{2}+\left(z-z^{\prime}\right)^{2}}
$$

Para simplificar en la Figura B.1 movemos el punto de observación al origen y suponemos que la densidad del cuerpo sea constante. La atracción vertical de la gravedad es dada por:

$$
g(P)=\frac{\partial U}{\partial z}=2 G \rho \iint \frac{z^{\prime} d x^{\prime} d z^{\prime}}{x^{\prime 2}+z^{\prime 2}}
$$

haciendo la integración sobre $x^{\prime}$ resulta:

$$
g=2 G \rho \int\left[\arctan \frac{x_{2}^{\prime}}{z^{\prime}}-\arctan \frac{x_{1}^{\prime}}{z^{\prime}}\right] d z^{\prime}
$$


B.
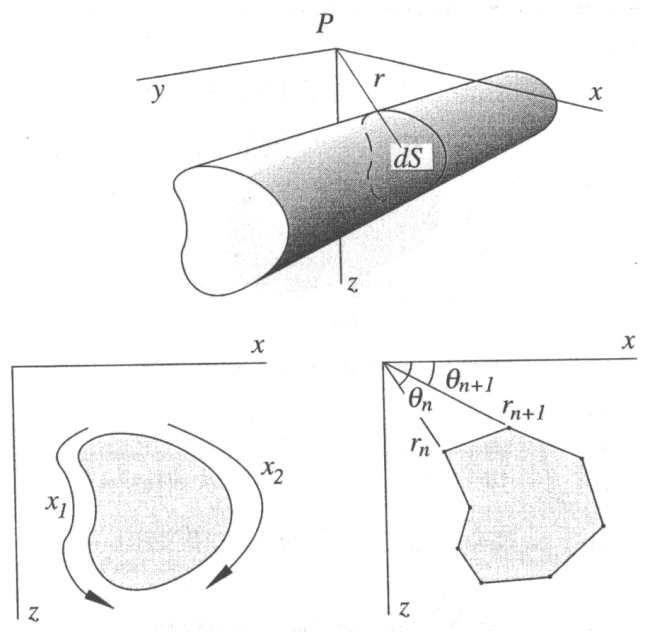

Figura B.1. Aproximación de un cuerpo bidimensional por un polígono de $\mathrm{N}$ lados, (modificado de [9]).

donde $x_{1}^{\prime}$ y $x_{2}^{\prime}$ son ambas funciones de $z^{\prime}$ y como se ven en la Figura B.1, representan caminos diferentes alrededor del perímetro de la sección transversal. Estos dos caminos al tomarse conjuntamente y considerando el cambio de signo, la integración en un circuito cerrado alrededor del perímetro es:

$$
g=2 G \rho \int \arctan \frac{x^{\prime}}{z^{\prime}} d z^{\prime}
$$

Ahora reemplazamos el perímetro con un polígono de $\mathrm{N}$ lados, por lo que la integral se convierte en:

$$
g=2 G \rho \sum_{n=1}^{N} \int_{z_{n}}^{z_{n+1}} \arctan \frac{x^{\prime}}{z^{\prime}} d z^{\prime}
$$

donde $z_{n}$ y $z_{n+1}$ son las coordenadas $z$ de dos puntos del lado $n$. Antes de continuar, necesitamos una expresión de x' en términos de z' y esto es dado por la ecuación de una recta:

$$
x^{\prime}=\alpha_{n} z^{\prime}+\beta_{n}
$$

donde

$$
\begin{aligned}
\alpha_{n} & =\frac{x_{n+1}-x_{n}}{z_{n+1}-z n} \\
\beta_{n} & =x_{n}-\alpha_{n} z_{n}
\end{aligned}
$$

Sustituyendo la ecuación B.7 en la ecuación B.6 y resolviendo la integral se obtiene:

$$
g=2 G \rho \sum_{n=1}^{N} \frac{\beta_{n}}{1+\alpha_{n}^{2}}\left[\log \frac{r_{n+1}}{r_{n}}-\alpha_{n}\left(\theta_{n+1}-\theta_{n}\right)\right]
$$

$r_{n}$ y $\theta_{n}$ están definidos como se ve en la Figura B.1, más detalles en Blakely, 1996 [9]. 


\section{Apéndice $\mathrm{C}$}

\section{C.1 Rutinas de GMT}

La rutina grdfft está dentro del paquete de software libre GMT (Generic Mapping Tools, 2007, (http://gmt.soest.hawaii.edu/ )), el cual consta de un manual con especificaciones de lo que se calcula en esta rutina como así también ejemplos de aplicación. A medida que se actualiza el software algunas opciones para su aplicación pueden modificarse. Describimos aquí las rutinas grdfft para la versión 4.5.6 y luego gravfft que fue obtenida desde la página de Joaquim Luis (http://w3.ualg.pt/ jluis/mirone/main.html), ya que al momento de usarla no estaba oficialmente incluida en el paquete de GMT, actualmente lo está (Generic Mapping Tools, 2013) y si estaba incluida en la versión de GMT mantenida por Joaquin Luis,

grdfft : lleva a cabo operaciones en grillas en el dominio de los números de onda (frecuencia). Lo aplicamos con la siguiente línea de comandos:

grdfft batimetría.grd -GWS.grd -S\$parker -C\$topoavg1 -L -M -V

donde: batimetria.grd es la grilla de la topografía

WS.grd es el nombre de la grilla de salida que contiene la contribución gravitacional de la interfaz calculada.

\$parker es una variable que contiene $2^{*} \mathrm{PI}^{*} \mathrm{G}^{*}$ (rload-rwater)*1.e5; el factor e5 es para pasar a mgal, rload es la densidad de los sedimentos y rwater es la densidad del agua. \$topoavg1 es el valor medio de la topografía para la "continuación hacia arriba" con la transformada de Fourier

-L es una opción que deja la serie como esta no restar ninguna tendencia.

-M elegir esta opción si su grilla es geográfica

-V opción para que cada vez que se ejecuta enumere por pantalla lo que se va ejecutando.

gravfft: Calcula la atracción gravitacional de superficies 3D en el dominio de los numeros de onda (o frecuencia).

gravfft topochichg11.grd -D $\$$ contr_dens -GWS2.grd -E $\$ \mathrm{n} \_\mathrm{p} \_$exp $-\mathrm{M}-\mathrm{V}$

donde: topochichg11.grd es la grilla de topografía.

WS2.grd es el nombre de la grilla de salida que contiene la contribución gravitacional de la interfaz calculada. 
C.

\$contr_dens= es el contraste de densidad.

$\$ \mathrm{n} \_\mathrm{p} \_$exp $=$es el número de términos que se usa en el desarrollo de parker.

-M elegir esta opción si su grilla es geográfica.

-V opción para que cada vez que se ejecuta el script liste por pantalla lo que se va ejecutando. 


\title{
Apéndice D
}

\author{
D.1 Perfiles de la anomalía suma para las grillas de \\ espesor Sedcombi y SedcombiCo
}


(a)

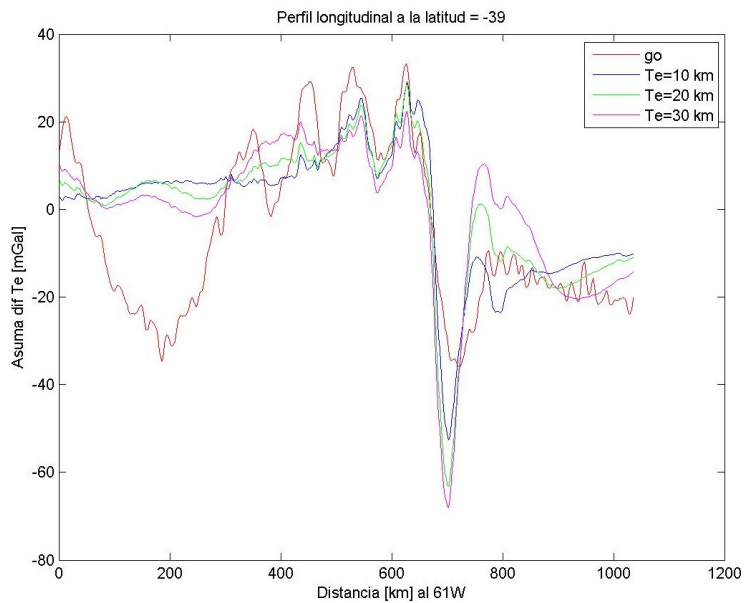

(b)

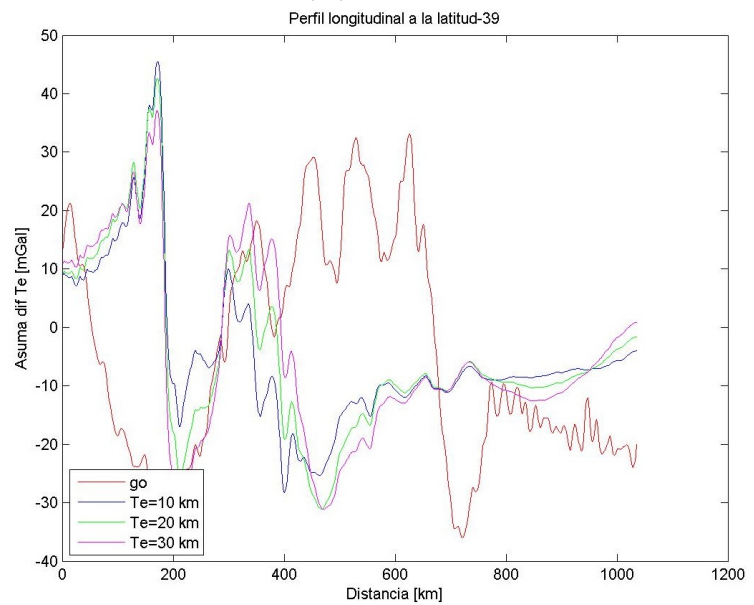

Figura D.1. Anomalía suma para el caso del perfil $39^{\circ} \mathrm{S}$ calculada para las grillas de espesor sedimentario Sedcombi (a) y SedcombiCo (b).

(a)

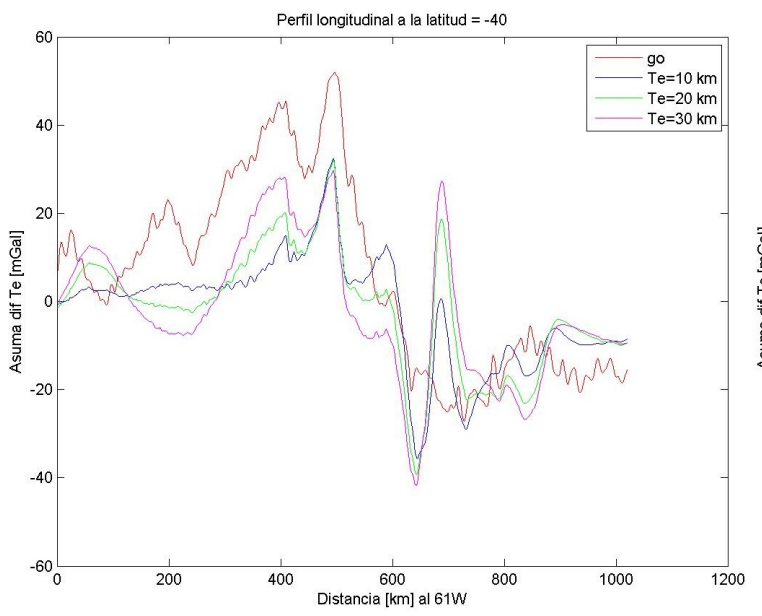

(b)

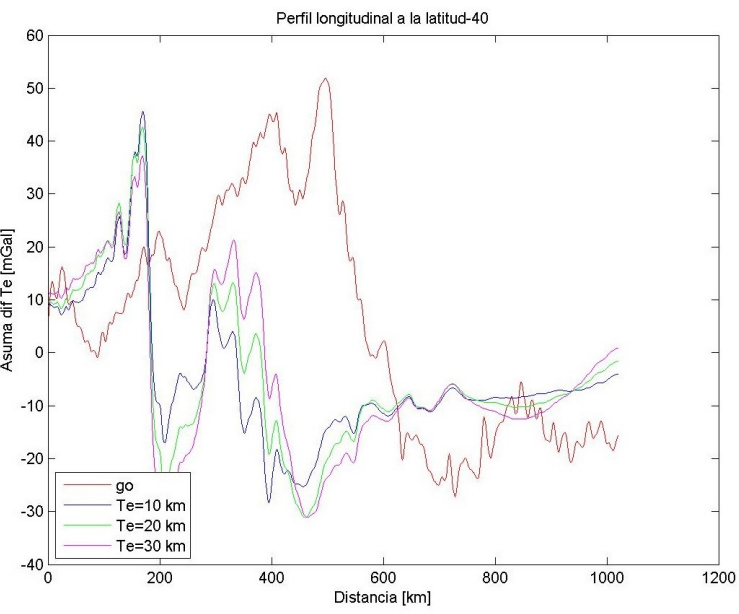

Figura D.2. Anomalía suma para el caso del perfil $40^{\circ} \mathrm{S}$ calculada para las grillas de espesor sedimentario Sedcombi (a) y SedcombiCo (b). 
(a)

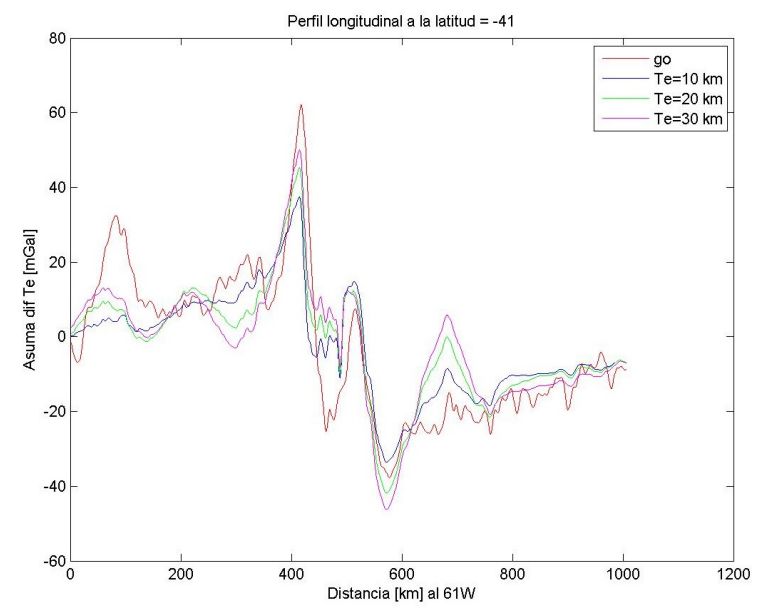

(b)

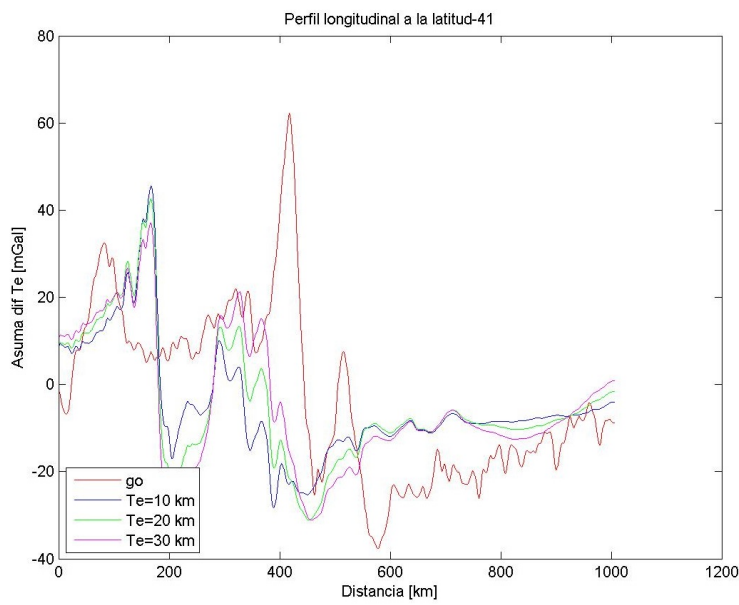

Figura D.3. Anomalía suma para el caso del perfil $41^{\circ} \mathrm{S}$ calculada para las grillas de espesor sedimentario Sedcombi (a) y SedcombiCo (b).

(a)

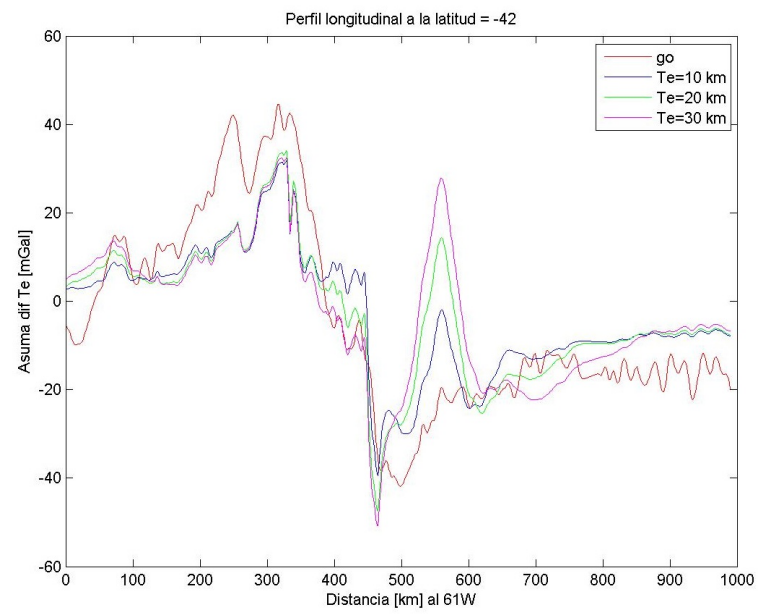

(b)

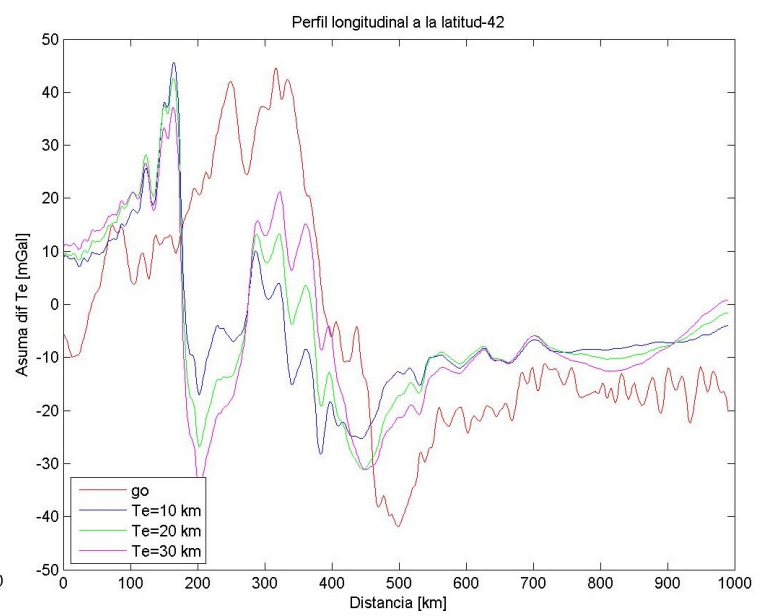

Figura D.4. Anomalía suma para el caso del perfil $42^{\circ} \mathrm{S}$ calculada para las grillas de espesor sedimentario Sedcombi (a) y SedcombiCo (b). 
D.

(a)

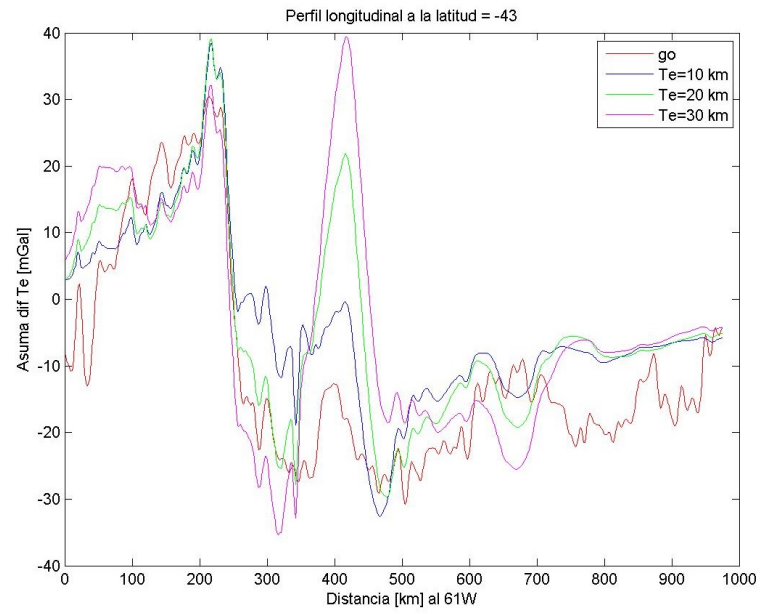

(b)

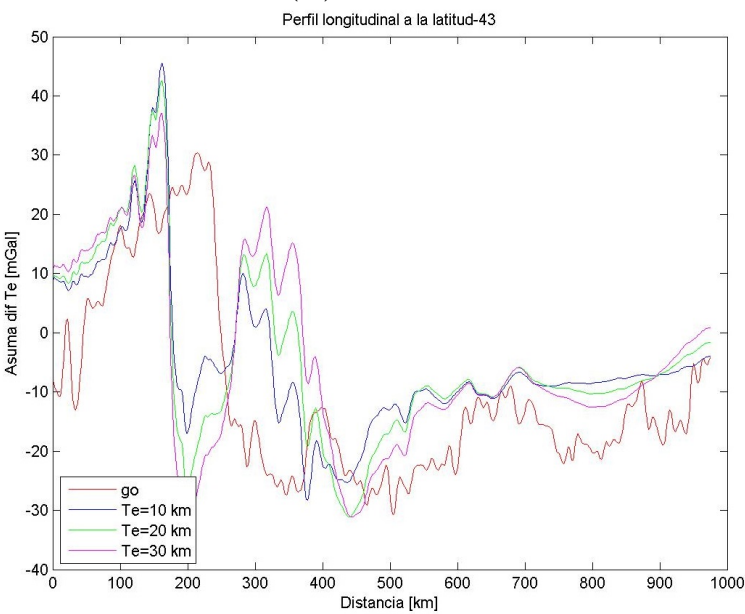

Figura D.5. Anomalía suma para el caso del perfil $43^{\circ} \mathrm{S}$ calculada para las grillas de espesor sedimentario Sedcombi (a) y SedcombiCo (b).

(a)

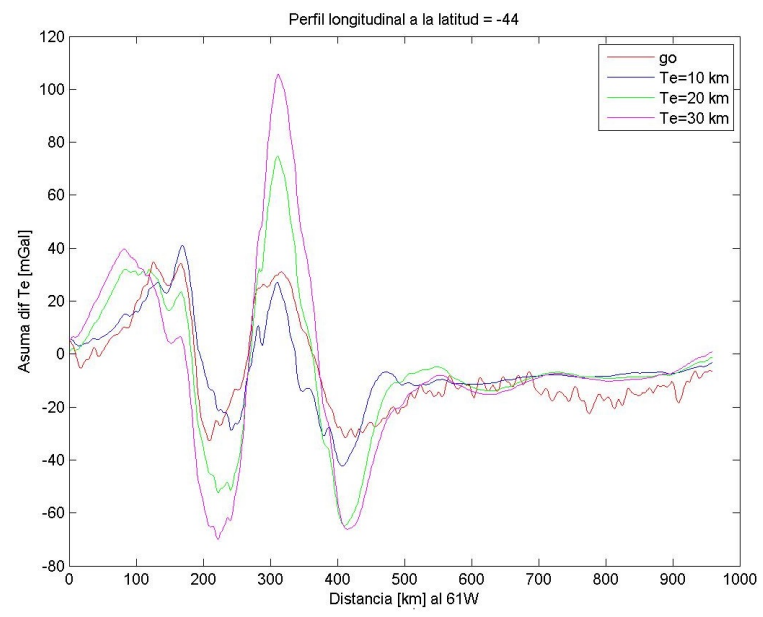

(b)

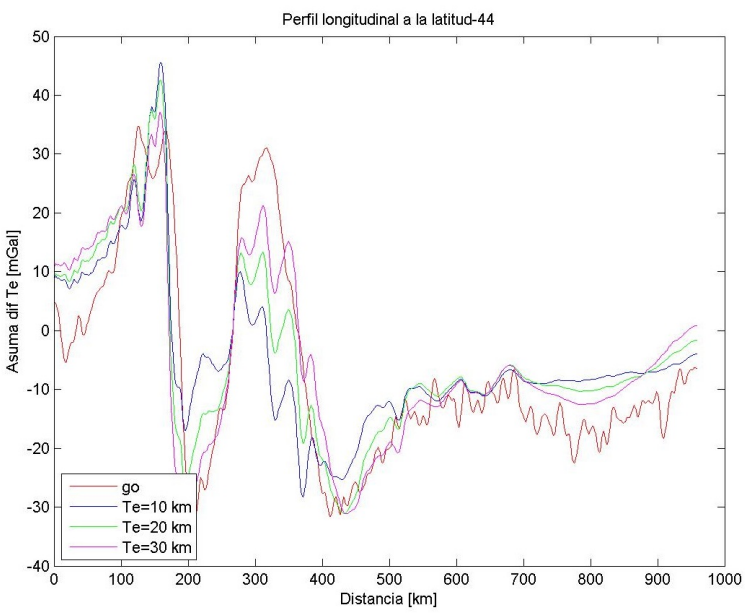

Figura D.6. Anomalía suma para el caso del perfil $44^{\circ} \mathrm{S}$ calculada para las grillas de espesor sedimentario Sedcombi (a) y SedcombiCo (b). 
(a)

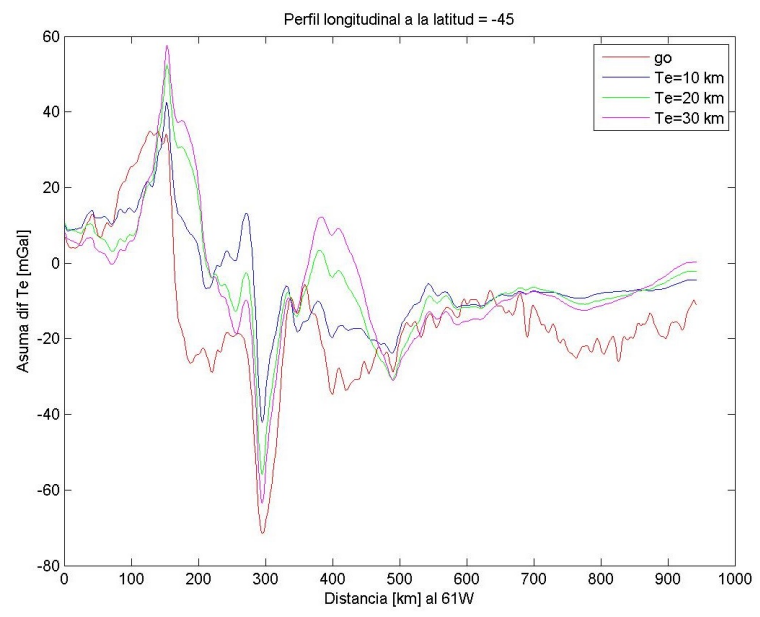

(b)

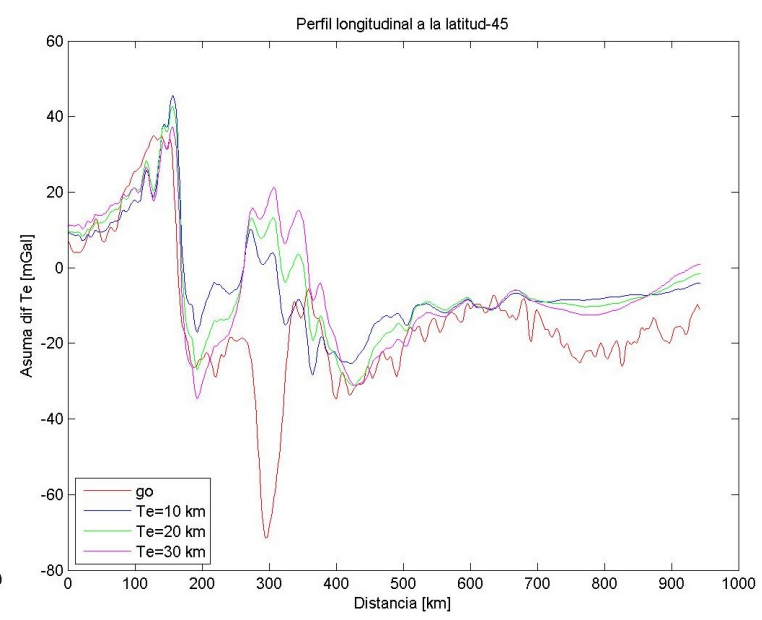

Figura D.7. Anomalía suma para el caso del perfil $45^{\circ} \mathrm{S}$ calculada para las grillas de espesor sedimentario Sedcombi (a) y SedcombiCo (b).

(a)

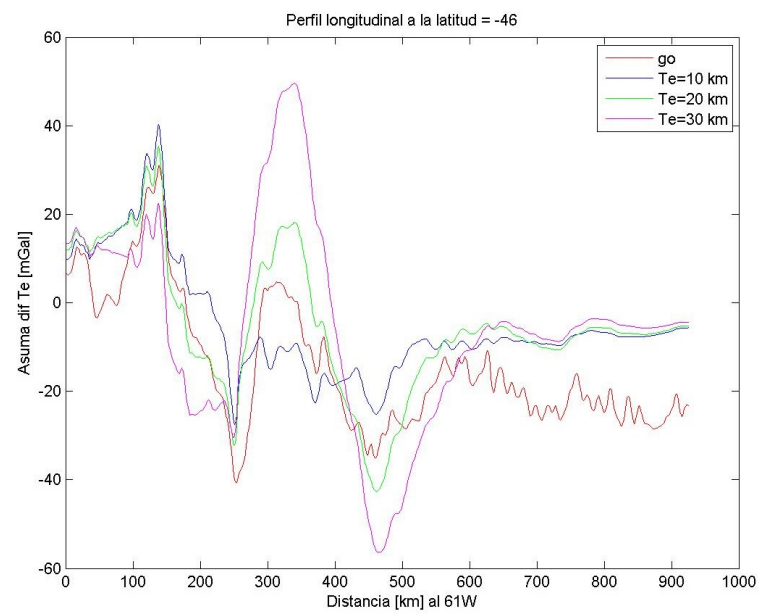

(b)

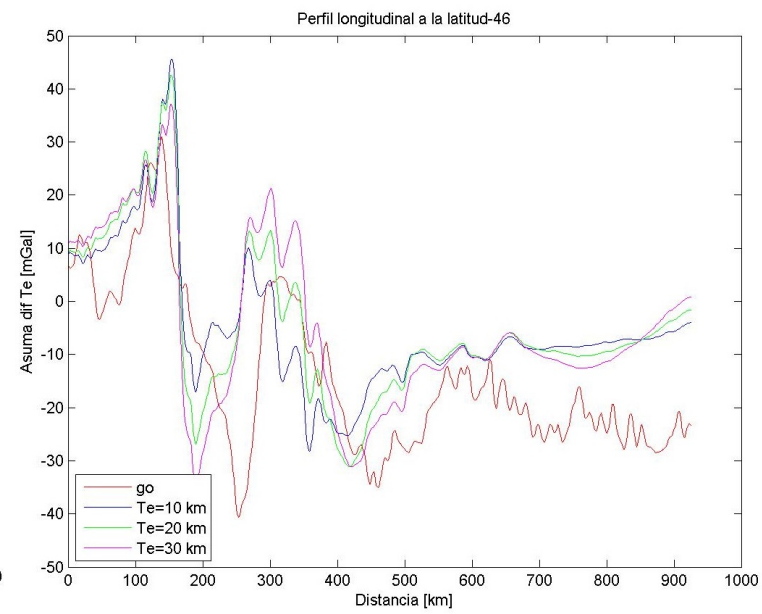

Figura D.8. Anomalía suma para el caso del perfil $46^{\circ} \mathrm{S}$ calculada para las grillas de espesor sedimentario Sedcombi (a) y SedcombiCo (b). 
D.

(a)

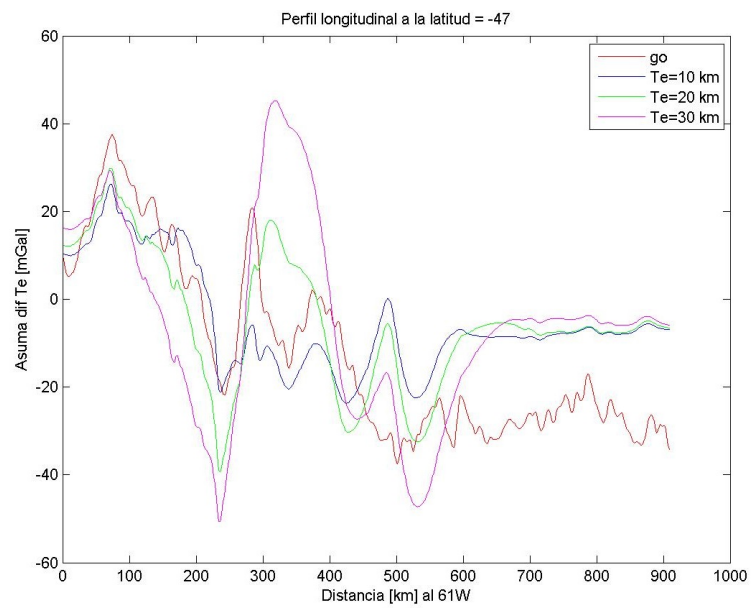

(b)

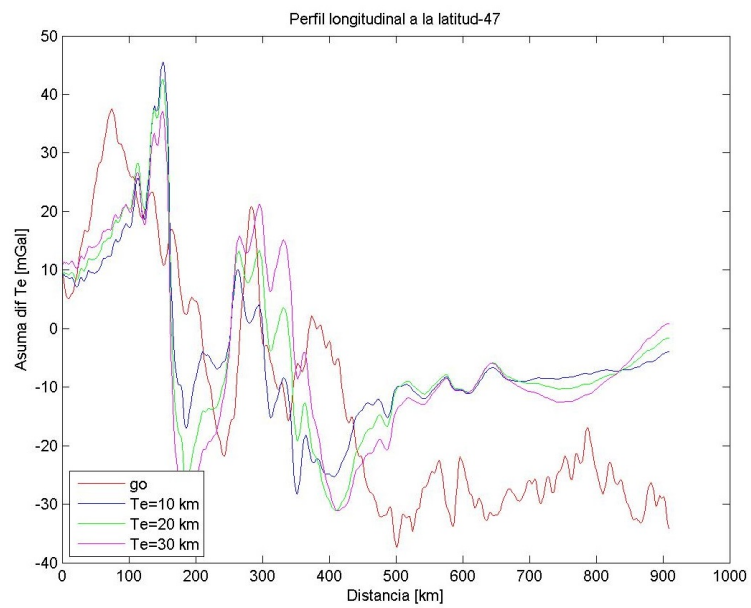

Figura D.9. Anomalía suma para el caso del perfil $47^{\circ} \mathrm{S}$ calculada para las grillas de espesor sedimentario Sedcombi (a) y SedcombiCo (b).

(a)

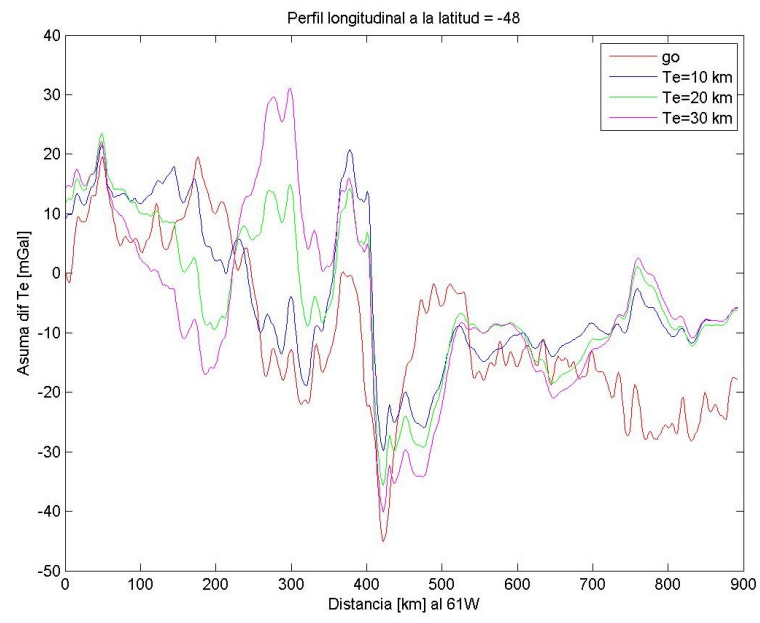

(b)

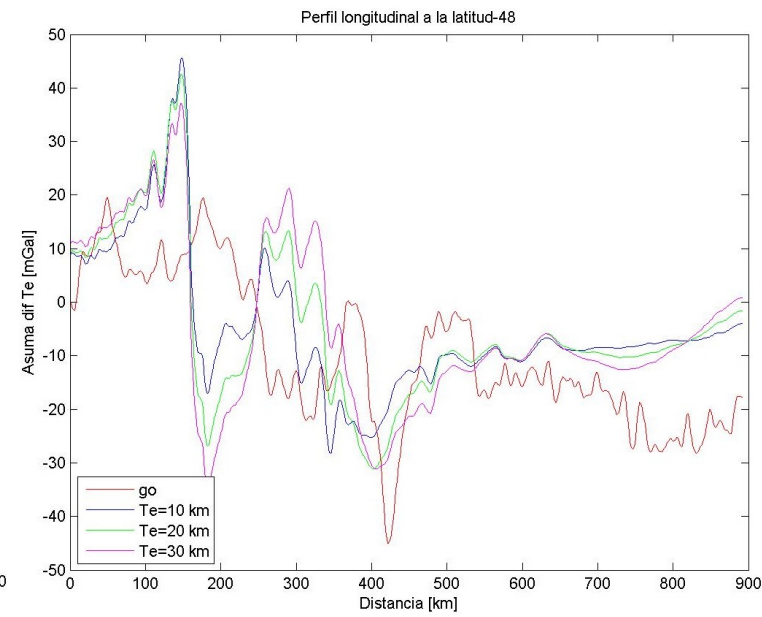

Figura D.10. Anomalía suma para el caso del perfil $48^{\circ} \mathrm{S}$ calculada para las grillas de espesor sedimentario Sedcombi (a) y SedcombiCo (b). 


\section{Bibliografía}

[1] Allen, P. A. and Allen, J. R., 1990. Basin Analysis. Principles and applications, Blackwell Science Ltd.

[2] Andersen, O. and Knudsen, P., 1998, The DNSC08 global mean sea surface and Bathymetry. Journal of Geophysical Research, 103n, C4, p. 8129-8137.

[3] Andersen, O. and Knudsen, P., 2008. Recent development in high-resolution global altimetric gravity field modeling. The leading edge, 2010.

[4] Andersen, O., Knudsen, P., Berry, P. and Kenyon, S., 2008, The DNSC08 ocean wide altimetry derived gravity field. Presentado EGU-2008,Viena, Austria, abril, 2008 .

[5] Andersen, O. and Knudsen, P., 2009. The DNSC08 mean sea surface and dynamic topography models. Journal of Geophysical Research, 114, c11001, doi:10.1029/2008jc005179.

[6] Beard, J., Ragland, C., 2005. Reactive bulk assimilation: A model for crust-mantle mixing in silicic magmas. Geology, 33, 681-684, doi:10.1130/G21470AR.1.

[7] Beaumont, C., Keen, C.E. and Boutilier, R., 1982a. On the evolution of rift continental margins: Comparison of models and observation for the Nova Scotia Margin. Geophys. J. R. Astron. Soc. 70, 667-715.

[8] Beaumont, C., Keen, C. E. and Boutilier, R., 1982b. Acomparison of forelands and rift margin sedimentary basins. Phil. Trans. R. Soc. London, A 305, p. 295-317.

[9] Blakely, R. J., 1996. Potential theory in gravity and magnetic applications, Cambridge University Press.

[10] Bott, M. H. P., 1980. Mechanism of subsidence at passive continental margins. In: Dynamics of Plate Interiors Am. Geophysical Union Geodynamics Series, 1, p. $27-32$.

[11] Braun, J. and Beaumont, C, 1987. Styles of continental rifting:results from dynamic models of lithospheric extension,. in Beaumont, C., and Tankard, A. J., eds., Sedimentary Basin and Basin-Forming Mechanism: Calgary, AB, Canadian Society of Petroleum Geologists, p. 241-258.

[12] Caminos R. y González, P. D.,1996. Mapa Geológico de la República Argentina. Publicación del Servicio Geológico Minero Argentino. 
[13] Campbell, I.H. and Griffiths, R. W., 1990. Implications of mantle plume structure for the evolution of flood basalts. Earth and Planetary Science Letters, vol. 99, p. 79-93.

[14] Cande, S.C., LaBrecque, J.L., Larson, R.L., Pitman, W.C., Golovchenko, X. and Haxby, W.F., 1989. Magnetic Lineations of the World's Ocean Basins. AAPG, Tulsa, OK, USA.

[15] Clift, P. D., Turner, J. and Ocean Drilling Program Leg 152, 1995. Dynamic support by the Icelandic plume and vertical tectonics of the northeast Atlantic continental margins: J. Gorphys. Res., vol. 100, p. 24473-24486

[16] Coffin M. F. and Eldholm, O., 1994. Large igneous provinces: crustal structure, dimensions, and external consequences, Reviews of Geophysics, 32, 1, p. 1-36.

[17] Corvetto C. B. and Introcaso, A., 2000. Isostatic analysis on an andean profile at $20^{\circ} \mathrm{S}$ using geoid undulations and gravity anomalies

[18] Coscia, C.A., 2000. Aprovechamiento de los datos sísmicos preexistentes para el análisis del espesor sedimentario en el margen continental argentino. En: Seminario de la Plataforma Continental, Buenos Aires, publicación del Consejo Argentino para las Relaciones Internacionales (CARI), p. 249-265.

[19] Cox, G. K., 1980. A model for flood basalt volcanism: J. Petrol, vol. 21, p. 629-650.

[20] Cuna T., 2008. Gravity Anomalies, Flexure and the Thermo-Mecanical Evolution of the West Iberia Margin and its Conjugate of Newfoundland. PhD Thesis, University of Oxford, Wolfson College \& Department of Earth Sciences.

[21] Dalziel, I. W. D. y Elliot D. H., 1971. Evolution of the Scotia Arc, Nature Publishing Group, p. 246-252.

[22] Deng, X., Featherstone, W. E., Hwang, C. and Shum, C. K., 2002. Improved Coastal Marine Gravity Anomalies at the Taiwan Strait from Altimeter Waveform Retracking, In: Proceeding of the international Workshop on Satellite Altimetry for Geodesy, Geophysics and Oceanography, Wuhan, 8-13 sept.

[23] Denker, H. and Roland, M., 2003. Compilation and evaluation of a consistent marine gravity data set surrounding Europe, Proc IUGG General Assembly, Sapporo, Japan, Springer, Berlin.

[24] Duncan, R. A. and Richards, M. A., 1991. Hot spots, mantle plumes, floods basalts, and true polar wander. Reviews of Geophysics, vol. 29, p. 31-50.

[25] Eagles, G., M. Konig, 2008. A model of plate kinematics in Gondwana breakup. Geophysical Journal International, 173, p. 703-717.

[26] Featherstone, W. E., 2003. Comparison of different satellite altimeter-derived gravity anomaly grids whith ship-borne gravity data around Australia, in: TZIAVOS, I. N. 8ED) gravity and Geoid 2002, Ziti editions, Thessaloniki, p. 326-331. 
[27] Fernandez-Molina F. J., Paterlini, M., Violante, R., Marshall, P., Somoza, L. and Rebesco, M., 2009. Contourite depositional system on the Argentine Slope: An exceptional record of the influence of Antartic wáter mases, Geology, vol. 37, no. 6, p. 507-510.

[28] Fowler, S., R., White, R. S., Spence, G. D., andWestbrook, G. K., 1989. The Hatton Bank continental margin- II. Deep structure from two-ship expanding spread seismic profiles:Geophys. J. Int., vol. 96, p. 295-309.

[29] Fragoso C. R., 1993. As placas brasilianas do sul e sudeste da plataforma sudamericana. IV Simposio Nacional de Estudios Tectonicos, Sociedade Brasileira de Geologia, p. 23-26. Belo Horizonte.

[30] Franke, D., Neben, S., Hinz, K., Meyer, H. and Schreckenberger, B., 2002. Hidrocarbon habitat of volcanic rifted passive margins. APG Hedberg conference.

[31] Franke, D. , Neben, S., Ladage, S., Schreckenberger, B., Hinz, K., 2007. Margin segmentation and volcano-tectonic architecture along the volcanic margin of Argentina/Uruguay, South Atlantic, Elsevier, Marine Geology, 244, 46-47.

[32] Franke, D., Ladage,S., Schnabel, M., Schreckenberger, B. , Reichert, C. , Hinz, K., Paterlini, M., de Abelleyra, J., and Siciliano, M., 2010. Birth of a volcanic margin off Argentina, South Atlantic, Geochem. Geophys. Geosyst., vol. 11, no. 2, Q0AB04, doi:10.1029/2009GC002715.

[33] Fu L. L., and Casenave, A., 2008. Satellite Altimetry and Earth Sciences: A Handbook of Techniques and Application. San Diego Academic Press, 463 p.

[34] Fullea, J., Fernandez, M. and Zeyen, H., 2000. FA2BOUG-A FORTRAN 90 code to compute Bouguer gravity anomalies from gridded free-air anomalies: Application to the Atalntic-Mediterranean transition zone, Computers \& Geosciences, vol. 34, p. $1665-1681$.

[35] Ghidella, M. E., Paterlini, M., Kovacs, L. C., Rodríguez, G., 1995. Magnetic Anomalies on the Argentine Continental Shelf. Actas del 4o Congreso Internacional de la Sociedad Brasileña de Geofísica $/ 1^{\mathfrak{l}}$ Conferencia Latinoamericana SEG/ULG realizado en Río de Janeiro del 20 al 24 de agosto, 8.

[36] Ghidella, M.E., Schreckenberger, B., Paterlini, C. M., Abraham, D.A., 2006. Anomalías magnéticas en el margen argentino. Actas en CD-ROM de la XIIIa. Reunión de Tectónica (San Luis, 16 - 20 octubre 2006), 4 pp, ISBN 978 - 987 - 1031 - 49 - 8.

[37] Ghidella, Köhn, M.E. y Gianibelli, J.C., 2002a. Low Altitude Magnetic Anomaly Compilation in Argentina: its Comparison with Satellite Data. American Gophysical Union, 2002 Spring Meeting. Publicado en: http://www.dsri.dk/multimagsatellites/

[38] Ghidella, M.E., Köhn, J., Gianibelli, J. C., Kostadinoff, J., y Chernicoff, C. J., 2002b. Compilación digital de datos magnéticos en Argentina. XXI Reunión Científica de Geofísica y Geodesia, Rosario. Actas AAGG2002 (CD ROM), p. 231-235. 
[39] Goméz-Ortiz D. and Argawal, B. N. P., 2005. 3DINVER.m a MATLAB program to invert the gravity anomaly over a 3D horizontal density interface by ParkerOldenburg's algorithm, Computers \& Geosciences, vol. 31, p. 513-520.

[40] Hackney, R. I. and Featherstone, W. E., 2003. Geodetic versus Geophysical Perspectives of the "Gravity Anomaly", Geophysical Journal International, no. 154, p. $35-43$.

[41] Hayes, D.E. and LaBrecque, J.L., 1991. Sediment isopachs: Circum-Antarctic to $30^{\circ} \mathrm{S}$. En: Marine Geological and Geophysica Atlas of the Circum-Antarctic to $30^{\circ} \mathrm{S}$. Antarctic Research Series. Editado por D.E. Hayes, AGU, Washington D.C., USA

[42] Heiskanen, W.A. and Moritz, H., 1967. "Physical Geodesy". W.H. Freeman, San Francisco.

[43] Hinz, K., 1981. A Hypothesis on Terrestrial Catastrophes. Wedges of Very Thick Oceanward Dipping Layers beneath Passive Continental Margins, Geologische Jahrbuch, vol. 22, Ingenta, Hannover.

[44] Hinz, K., 1981. Wedges of very thick oceanward dipping layers beneath passive continental margins. The origin and paleoenvironmental significance. Geologisches Jahrbuch E, vol. 22, p. 3-28.

[45] Hinz, K., S. Neben, Schreckenberger, B. , Roeser, H. A., Block, M., Goncalves de Souza, K. and Meyer, H., 1999. The Argentine continental margin north of $48^{\circ} \mathrm{S}$ : sedimentary successions, volcanic activity during breakup. Marine and Petroleum Geology 16, p. 1-25.

[46] Holbrook, W.S. y Kelemen, P.B., 1993. Large Igneous Province on the US Atlantic Margin and Implications for Magmatism During Continental Breakup, Nature, 364, Nature Publishing Group, p. 433-436.

[47] Hubbert, M. K., 1948. A line-integral method of computing the gravimetric effects of two-dimensional masses, Geophysics, no. 13, p. 215-225.

[48] Introcaso, A. y Ramos, V. 1984. La cuenca del Salado. Un modelo de evolución aulacogénica. 9o Congreso Geológico Argentino. Actas 3, p. 27-46.

[49] Karner, G.D. and Watts, A.B., 1982. On Isostasy at Atlantic ${ }^{`}$ Type Continental Margins, J. Geophys. Res., vol. 87, no. B4, p. 2923-2948.

[50] Kelemen P. B., and Holbrook, W. S., 1995. Origin of thick, high-velocity igneous crust along the East Coast Margin: J. Geophys. Res., vol. 100, p. 10077-10094.

[51] Keller, G. R., Hildenbrand, T. G., Hinze, W. J., Li, X., Ravat, D., Webring, M., 2006. The quest for the perfect gravity anomaly: Part 2 - - Mass effects and anomaly inversion, SEG Expanded Abstracts 25, p. 864-868.

[52] LaFehr, T. R., 1991. An exact solution for the gravity curvature, Bullard B, Geophysics, vol. 56, no. 8, p. 1179-1184. 
[53] Lemoine, F. G., Kenyon, S. C., Factim, J. k., Trimmer, R. G., Pavlis, N. K., Chinn, D. S., Cox, C. M., Klosko, S. M., Luthcke, S. B., Torrence, M. H., Wang, Y. M., Williamson, R. G. , Pavlis, E. C., Rapp, H. and Olson, T. R., 1998. The Development of the Joint NASA GSFC and the National IMagery and Mapping Agency (NIMA) Geopotential Model EGM96; NASA Technical Paper NASA/TP1998206861, Goddard Space Flight Center, Greenbelt, USA.

[54] Light, M. P. R., Keeley, M. L., Maslanyj, M. P., y Urien, C. M., 1993. The Tectonostratigraphic Development of Patagonia, and its Relevance to Hydrocarbon Exploration, Journal of Petroleum Geology, vol. 16, N 4, p. 465-482.

[55] Lister, G. S., Etheridge, M. A. and Symonds, P. A., 1991. Detachment models for the formation of passive continental margins. Tectonics, 10, 1038-1064.

[56] Ludwig, W. J., Carpenter, G., Houtz, R. E., Lonardi, A. G., Ríos, F. F., 1978. Sediment isopach map of the Argentine Continental Margin, Argentine Shelf, Argentine Basin and Falkland Plateau. American Association of Petroleum Geologists, Tulsa, Oklahoma 74101.

[57] Luis, J. F. and Miranda, J. M., 2008. Revaluation of magnetic chrons in the North Atlantic between $35 \mathrm{~N}$ and $47 \mathrm{~N}$ : Implications for the formation of the Azores Triple Junction and associated plateau. J. Geophys. Res., 113, B10105 [potential supplement]

[58] Max, M. D., Ghidella, M. E., Kovacs, L., Paterlini, M. Valladares, J. A., 1999. Geology of the mainland Argentine continental shelf and margin from aeromagnetic survey. Marine Petroleum Geology, 16, p. 41-64.

[59] McKenzie, D., 1978. Some remarcks on the development of sedimentary basin. Earth and Planetary Sci. Lett., 40, p. 25-32.

[60] McMillan, W. G., 1958. The theory of Potential. Dover Publisher, New York, p. 469.

[61] Menzies M.A., .Klemperer, S.L, Ebinger, C. J., Baker, J., 2002. Characteristics of volcanic rifted margins, Geological Society of America Special Paper, 362, p. 1-14

[62] Moulin, M., Aslanian, D. and Unternehr, P., 2010. A new starting point for the South and Equatorial Atlantic Ocean, Earth-Science Reviews, 98, p. 1-37.

[63] Mutter, J. C., Talwani M., and Soffa, P. L., 1982. Origin of seaward-dipping reflectors in oceanic crust off the Norwegian Margin by "subareal seafloor spreading". Geology, vol. 10, p. 353-357.

[64] Mutter, J. C., BUCK, W. R. y ZEHNDER, C. M., 1988. Convective Partial Melting - A Model for the Formation of Thick Basaltic Sequences during the Initiation of Spreading, en Journal of Geophysical Research, vol. 93, N B2, pp. 1031-1048.

[65] Nagy, D., Papp, G. and Benedek, J., 2000. The gravitational potential and its derivatives for the prism, Journal of Geodesy, vol. 74, p. 552-560 
[66] Neben, A., Franke, D., Hinz, K., Schreckenberger, B., Meyer, H. and Roeser, H. A., 2002. Early Opening of the South Atlantic: Pre-Rift Extension and Episodicity of Seaward Dipping Reflector Sequence (SDRS) Emplacement on the Conjugate Argentine amd Namibia Continental Margins. AAPG Hedberg Conference: Hydrocarbon Habitat of Volcanic Rifted Passive Margins. Stavanger, Noruega. Actas.

[67] Oldenburg D. W., 1974. The inversion and interpretation of gravity anomalies, Geophysics, vol. 39, no. 4, p. 526-536

[68] Ortiz, D. G. and Agarwal, B. N. P., 2005. 3DINVER.m a MATLAB program to invert the gravity anomaly over a 3D horizontal density interface by ParkerOldenburg's algorithm, Computer \& Geosciences, vol. 31, p. 513-520

[69] Pavlis, N. K., Holmes, S.A, Kenyon, S. C., and Factor, J. K, 2008. An earth Gravitational Model to degree 2160 :EGM2008, presented at the 2008 general assembly of the European Geosciences Union, Viena, Austria, April 13-18, (pdf file whit the original power point presentation).

[70] Parker R.L., 1973. The rapid calculation of potencial anomalies, Geophys. J. R. astr. Soc. , 31, p. 447-455.

[71] Parker C., Paterlini M. y Violante R. A., 1996. Compilación de datos sísmicos y determinación de isopacas en el Mar Argentino. Trabajo inédito, contribución al Mapa Geológico de la República Argentina.

[72] Pedraza De Marchi A. C., Tocho C. y Ghidella M. 2012. Comparación de anomalías de gravedad derivadas de altimetría satelital con datos de gravedad marina en el margen continental argentino. Boletim de Ciencias Geodésicas, Vol. 18, No. 1.

[73] Pedraza De Marchi A. C., Ghidella M. y Tocho C. 2014. Analysis of Different Methodologies to calculate Bouguer Gravity Anomalies in the Argentine Continental Margin. Geosciences. Scientific Academic Publishing, Vol. 4, No. 2.

[74] Pedraza De Marchi A. C., Ghidella M. y Tocho C. 2015. Esquema de tres interfaces con compensación isostática local en el margen continental argentino. Geociencias Aplicadas Latinoamericanas. EAGE, en proceso de revisión.

[75] Planke, S., Alvestad, E. and Eldholm O.1999. Seismic characteristics of basaltic extrusive and intrusive rocks. The Leading Edge vol. 18, no. 3, p. 342-348.

[76] Rabinowitz, P. D. and LaBrecque, J., 1979. The Mesozoic South Atlantic ocean and evolution of its continental margins. Journal of Geophysical Research 84, p. 5973-6002.

[77] Ramos, V. A., 1988. Late Proterozoic-Early Paleozoic of South America- colisional history. Episodes 118 (3):168-173, Otawa.

[78] Ramos, V. A., 1996. Evolución tectónica de la plataforma continental. En V. A. Ramos y M. A. Turic (Eds.) Geología y Recursos Naturales de la Plataforma Continental Argentina. XIII Congreso Geológico Argentino y III Congreso de Exploración de Hidrocarburos, Relatorio 21: 385-404. 
[79] Sandwell, D.T. and Smith, W. H. F, 1997. Marine Gravity from Geosat and ERS 1 Satellite Altimetry. J. Geophys. Res., vol. 102, no. B5, p. 10039-10054.

[80] Sandwell, D.T. , 1999. Chapter 11-Bathymetric estimation, Satellite Altimetry and Earth Sciences, Academic Press.

[81] Sandwell, D., 2009. Global marine gravity from retracked Geosat and ERS-1 altimetry:Ridge segmentation versus spreading rate, Journal of Geophysical Research, vol. 114, B01411, doi:10.1029/2008JB006008.

[82] Schmidt S., 2004. The Pretroleum Potential of the Passive Continental Margin of South-Western Africa - A Basin Modelling Study. PhD Thesis, Von der Fakultät für Georessourcen und Materialtechnik der Rheinisch-Westfälischen Technischen Hochschule Aachen.

[83] Sleep, N. H., 1971. Gravity modeling of the ocean-continent transition along the South Atlantic margins, Geophys. J. R. Astromom. Soc., 24, p. 325-350.

[84] Stavar, D. and Hall, S., 2009. Gravity modeling of the ocean-continent transition along the South Atlantic margins, J. Geophys. Res., doi:10.1029/2008JB006014, p. $1-46$.

[85] Steckler, M. S. and Watts, A. B., 1978. Subsidence of the Atlantic-type contienetal margin off New York. Earth planet. Sci. Lett., no.41, p. 1-13.

[86] Stewart, J., Watts, A. B. and Bagguley, J. G., 2000. Three-dimensional subsidence analysis and gravity modelling of the continental margin offshore Namibia. Geophys J. int., no. 141, p. 724-746.

[87] Talwani, M., Worzel, J. L. and Landisman, M., 1959. Rapid gravity computations for two-dimensional bodies with application to the Mendocino submarine fracture zone, Journal of Geophysical Research, no. 64, p. 49-59.

[88] Thybo, H and I. M. Almeida., 2013. Moho and magmatic underplating in continental lithosphere. Tectonophysics, 609, p. 605-619

[89] Turcotte, D. L and Schubert, J., 2002. Geodynamics, Cambridge University Press, 2002, XIII, 456 págs.

[90] Vargas Hernandez H. M., 2003. Análisis y comparación de la subsidencia entre la cuenca ibérica y ebro central durante el pérmico y triásico y su relación con el relleno sedimentario. Tesis Doctoral, Universidad Complutence de Madrid. Facultad de Ciencias Geológicas. Departamento de Estratigrafía.

[91] Vening Meinnesz, F. A., 1931. Une nouvelle methode pour la réduction isostatique régionale de l'intensité de la pesanteur. Bull. Géodésique, vol. 29, p. 33-51.

[92] Violante, R., Paterlini, C. M., P. Costa, Hernadez Molina F. J., Cavallotto, J. L., Marcolini, S., Bozzano, G. , Laprida, C., García Chapori, N., Bickert, T. and Volkhard, S., 2010. Sismoestratigrafía y evolución geomorfológica del talud continental adjacente al litoral del este bonaerence, Argentina, Latin American Journal of Sedimentology and Basin Analysis, vol. 17, no. 1, p. 33-62 
[93] Walcott, R.I., 1970b. Flexure the lithosfere at Hawaii. Tectonophysics, vol.9, p. 435-446.

[94] Walcott, R.I., 1970c. Isostatic response to loading of the crust in Canada. Can. J. Earth Sci, vol.7, p. 931-937.

[95] Walcott, R.I., 1972. Gravity, flexure and the growth of sedimentary basins at continental edge. Geol. Soc. Am. Bull. 83, p. 1845-1848.

[96] Watts, A. B. and Ryan, W. B. F., 1976. Flexure of the litosphere and continental margin basins, Tectonophysics, no. 36, p. 25-44.

[97] Watts, A. B., 1981. The U. S. Atlantic Continental Margin:Subsidence History, Crustal Structure and Thermal Evolution, CN 19: Geology of Passive Continental Margins: History, Structure and Sedimentologic Record (with Special Enphasis on the Atlantic Margin), AAPG Special Volumes, A123, p. 1-75.

[98] Watts, A. B. 1982. Tectonic subsidence, flexure and global changes of sea-level. Nature, 297, p. 469-474.

[99] Watts, A. B. 1988. Gravity anomalies, crustal structure and flexure of the lithosphere at the Baltimore Canyon Trough. Earth and Planetary Sci. Lett., 89, p. 221-238.

[100] Watts, A. B. and Stewart, J., 1998. Gravity anomalies and segmentation of the continetal margin offshore West Africa, Earth and Planetary Science Letters, no. 156, p. 239-252.

[101] Watts, A. B. and Fairhead, J. D., 1997. Gravity anomalies and magmatism at the British Isles continental margin: J. Geol. Soc. London, vol. 154, p. 523-529.

[102] Watts, A. B. and Fairhead, J. D., 1999. A process-oriented approach to modeling the gravity signature of continental margins, The Leading Edge, no. 18, p. 258-263.

[103] Watts, A. B., 2001. Isostasy and Flexure of the Lithosphere, Cambridge University Press.

[104] Watts, A. B., Rodger, M., Peirce, C., Greenroyd, C. J., and Hobbs, R. W., 2008. Seismic structure, gravity anomalies, and flexure of the Amazon continental margin, NE Brazil, J. Geophys. Research, vol. 114, B07103, doi:10.1029/2008JB006259.

[105] Wernicke, B., 1985. Uniform-sense normal simple shear of the continental lithosphere. Can. Journal of Earth Sci., 22, p. 22,108-22,125.

[106] Wessel P. and Smith, W. H. F., 2007. The Generic Mapping Tools Technical Reference and Cookbook, Version 4.2.0, p. 155.

[107] Wessel P., Smith, W. H. F., Scharroo, R., Luis, J. L. and Wobbe, F., 2013. The Generic Mapping Tools: Improved version released. EOS Trans. AGU, 94, 409-410. 
[108] White R. and McKenzie, D. ,1989. Magmatism at rift zones: The generation of volcanic continental margins and floods basalts, J. Geophys. Research, vol. 94, no. B6, p. 7685-7729.

[109] White R. S.,1992. Crustal structure and magmatism of Noth Atlantic continental margins:J. Geol. Soc L ond., vol. 149, p. 841-854.

[110] Zalán, P.V., Severino, M.C.G., Rigoti, C., Magnavita, L.P., Oliveira, J.A.B., and Viana, A.R., 2011. An entirely new 3D-view of the crustal and mantle structure of a ruptured South Atlantic passive margin Santos, Campos, and Espírito Santo Basins, Brazil: AAPG Search and Discovery, Article \#30177 (Expanded Abstract). , accseso al sitio 20 de Dic. de 2014. http://www.searchanddiscovery.com/documents/2011/30177zalan/ndx_zalan.pdf

[111] Zalán, P.V., 2013. Unthinkable Physical Analogs for the Modern Concepts on Continental Stretching and Rupturing. AAPG Search and Discovery, Article \#41128 (Adapted from extending Abstract prepared with oral presentation at AAPG Anual Convention and Exibition, Pittsburg, Pennsylvania, 19-22 de Mayo de 2013)

[112] Zambrano, J. J., V. M. Urien, 1970. Geological outline of the basins in Southern Argentina and their continuation off the Atlantic Shore, J. Geophys. Research, 75, no 8., p. 1363-1396. 